\title{
Methods for Geochemical Analysis
}
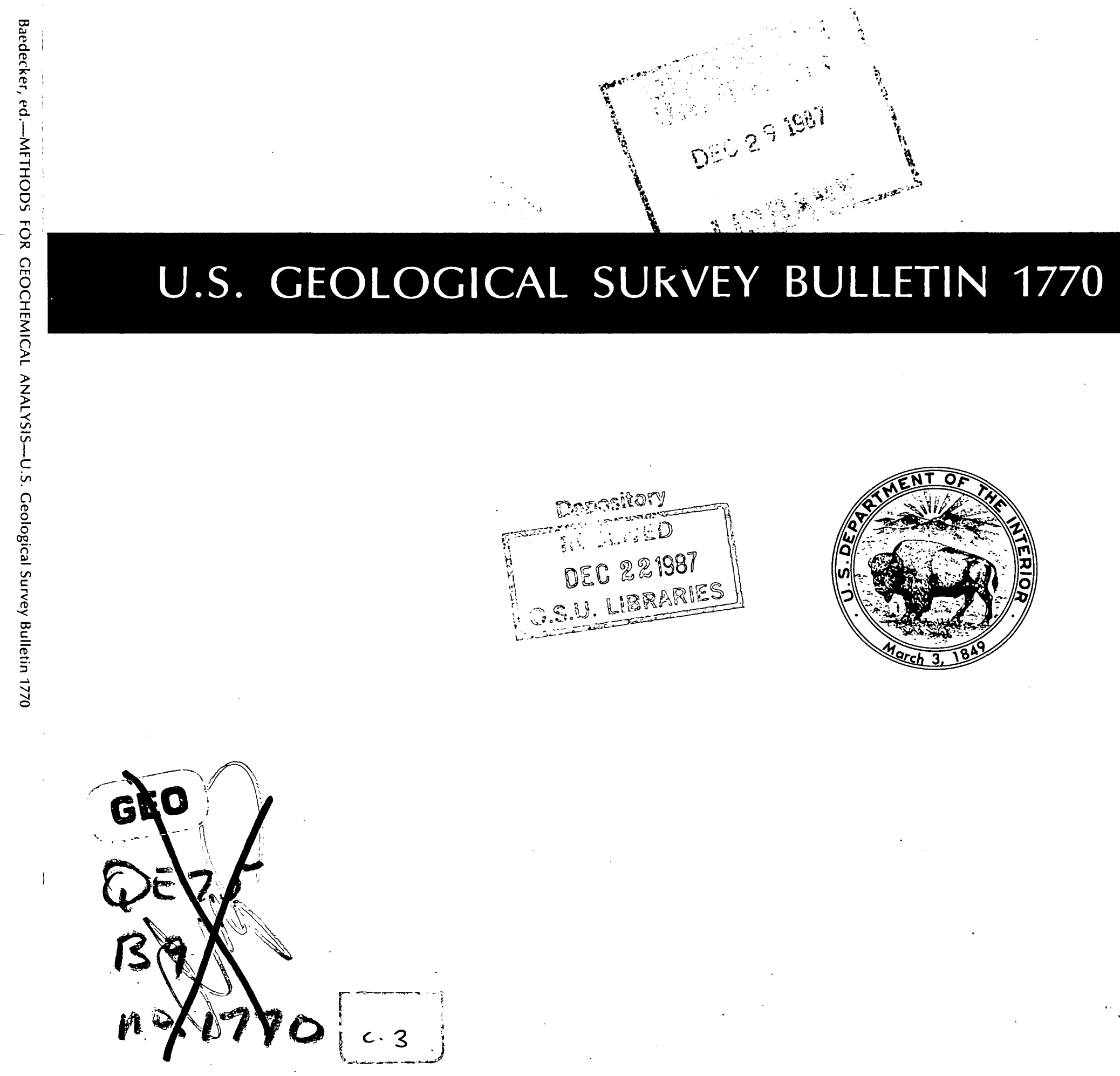



\section{Methods for Geochemical Analysis}

Edited by PHILIP A. BAEDECKER

Analytical methods used in the Geologic

Division laboratories of the U.S.

Geological Survey for the inorganic

chemical analysis of rock and mineral

samples 


\section{DEPARTMENT OF THE INTERIOR DONALD PAUL HODEL, Secretary}

\section{U.S. GEOLOGICAL SURVEY}

Dallas L. Peck, Director

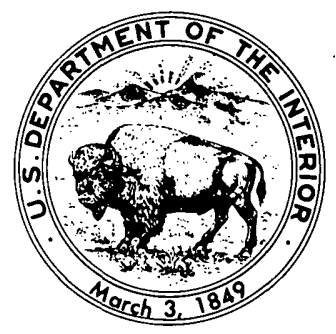

For sale by the Books and Open-File Reports Section, U.S. Geological Survey, Federal Center, Box 25425, Denver, CO 80225

\section{Llbrary of Congress Cataloging in Publication Data}

Methods for geochemical analysis.

(U.S. Geological Survey bulletin ; 1770)

Bibliography: $p$.

Supt. of Docs. no.: | 19.3:1770

1. Geochemistry, Analytic. I. Baedecker, Philip A. II. Series.

QE75.B9 no. 1770 [QE516.3] $557.3 \mathrm{~s} \quad 87-600387$ [551.9] 


\section{CONTENTS}

INTRODUCTION

P. A. Baedecker

IN1

CHAPTER A

Analysis of geologic materials by direct-current arc emission spectrography and spectrometry

D. W. Golightly, A. F. Dorrzapf, Jr, R. E. Mays, T. L. Fries, and N. M. Conklin

\section{CHAPTER B}

Inductively coupled plasma-atomic emission spectrometry

B1

F. E. Lichte, D. W. Golightly, and P. J. Lamothe

CHAPTER C

Atomic absorption methods

P. J. Aruscavage and J. G. Crock

C1

CHAPTER D

Chemical methods of separation for optical emission, atomic absorption spectrometry, and colorimetry

S. A. Wilson, J. S. Kane, J. G. Crock, and D. B. Hatfield

CHAPTER E

Analysis of geologic materials by wavelength-dispersive X-ray fluorescence spectrometry

J. E. Taggart, Jr, J. R. Lindsay, B. A. Scott, D. V. Vivit, A. J. Bartel, and K. C. Stewart

CHAPTER F

Energy-dispersive X-ray fluorescence spectrometry

F1

R. G. Johnson and B.-S. L. King

CHAPTER G

Major and minor elements requiring individual determination, classical whole rock analysis, and rapid rock analysis

D1

L. L. Jackson, F. W. Brown, and S. T. Neil

CHAPTER $\mathrm{H}$

Instrumental neutron activation analysis of geochemical samples

H1

P. A. Baedecker and D. M. McKown

\section{CHAPTER I}

Determination of uranium and thorium by delayed neutron counting

D. M. McKown and H. T. Millard, Jr.

\section{CHAPTER J}

Radiochemical neutron activation analysis of geologic materials

G. A. Wandless

CHAPTER K

Isotope-dilution mass spectrometry

J. A. Philpotts

Any use of trade names is for descriptive purposes only and does not imply endorsement by the U.S. Geological Survey 


\section{INTRODUCTION}

\section{By P. A. Baedecker}

The laboratories for analytical chemistry within the Geologic Division of the U.S. Geological Survey are administered by the Office of Mineral Resources. The laboratory analysts provide analytical support to those programs of the Geologic Division that require chemical information and conduct basic research in analytical and geochemical areas vital to the furtherance of Division program goals. Laboratories for research and geochemical analysis are maintained at the three major centers in Reston, Virginia, Denver, Colorado, and Menlo Park, California. The Division has an expertise in a broad spectrum of analytical techniques, and the analytical research is designed to advance the state of the art of existing techniques and to develop new methods of analysis in response to special problems in geochemical analysis. The geochemical research and analytical results are applied to the solution of fundamental geochemical problems relating to the origin of mineral deposits and fossil fuels, as well as to studies relating to the distribution of elements in varied geologic systems, the mechanisms by which they are transported, and their impact on the environment.

In 1984, a review of the role of analytical chemistry within the Geologic Division was conducted by an ad hoc committee of senior Division managers. The committee concluded that a lack of familiarity on the part of those submitting samples for geochemical analysis with the wide range of analytical techniques available could result in the misapplication of some techniques to various geochemical problems; for example, highly precise analyses could be applied to problems for which only approximate answers are needed or can be obtained in other ways. Conversely, investigators might request too few analyses or data by techniques that are not precise enough for the problem at hand; for example, potential waste occurs when data on a desired element, such as cesium, is obtained by requesting a broad-spectrum Instrumental Neutron Activation Analysis, which requires perhaps an order of magnitude more time and effort than if cesium alone were determined by the same method or possibly another. In addition to these concerns, analytical chemists often are concerned that those who use analytical data have little appreciation for the factors that affect the quality of that data and the challenges and complexity of analytical chemistry as a scientific discipline. The committee report stated, "...clearly, an increased understanding of modern analytical techniques by all potential users of chemical data in the Geologic Division is a highly desirable goal. Toward that end we are recommending that a publication entitled Methods for Geochemical Analysis be prepared, which will cover all aspects of the techniques currently available." This volume is in response to that recommendation.

In many respects, the current volume supplants two previous publications that were designed to familiarize scientists within the Geologic Division with the analytical capabilities that were (or are) available in the laboratories of the Division. The first edition of the Manual of Laboratory Services of the Division's Geochemistry and Petrology Branch was published in 1956. That document was subsequently revised in 1974 by the Office of Geochemistry and Geophysics. This volume is more restricted than either of the above publications in that the previous reports contained sections on geochronology, electron optics, $\mathrm{X}$-ray crystallography and diffraction, mineralogic and petrographic analysis, organic analysis, thermodynamics, geophysical methods, and so forth; the coverage offered by this volume, however, is limited to modern methods of inorganic analysis. In the preparation of Methods for Geochemical Analysis, the authors also have adopted a different approach to the subject matter. Rather than to provide a simple documentation of available services, they have attempted to treat each topic in much greater depth. Thus, each chapter contains sections on fundamental principles, an overview of the method as practiced in each center, the limitations of the technique (such as matrix, chemical, and spectral interferences), and the sensitivity, precision, and accuracy of each technique. The goal was to prepare a single volume that would provide the nonchemist reader with a broad coverage of geochemical analysis as a scientific discipline, a good understanding of the inherent difficulty of analyzing complex geologic matrices that are highly variable in composition, and sufficient technical detail to understand the factors that can affect the quality (precision and accuracy) of the analysis of the geologic sample. To again 
quote the 1984 analytical chemistry review report, "It is hoped that a widespread familiarization with this document by members of the Division will provide the basis for a more informed dialogue on chemical problems between geologists and analytical chemists."

At this point, I think it worthwhile to repeat a few sections from the introduction to the 1956 Manual of Laboratory Services because, although the technology of analytical chemistry has changed dramatically, the following general comments regarding routine and nonroutine analysis and analytical accuracy remain as valid today as they were 30 years ago:

The service work of the Branch falls into two categories, routine and research (including custom), which are neither completely nor even too sharply separated at any time. Depending on the circumstances, a routine determination may become a research problem, or what was originally a research problem may in time become a routine process. The Branch is constantly testing new methods with the aim of developing rapid routine determinations as the geologists indicate their need for them....Research determinations are those made by methods we do not ordinarily use; they may include determinations made on samples of unusual composition, or the analysis for some element or elements at lower concentration ranges than previously handled, or the development of new chemical, spectrographic, mineralogical, X-ray, or other methods. They may involve adaptation of existing methods and apparatus. Study and judgment are required to select the best method for the specific problem, standards must be set up to insure the validity of the results, and much time spent in checking possible interferences. Research work of this type may result in the development of new lines of attack on unsolved problems.

The following general comments on the analytical accuracy are also still applicable:

To conserve laboratory effort, it is equally important not to request excessive accuracy and sensitivity; the accuracy of the analysis need be no better than the sampling techniques and the use which will be made of the analyses [my emphasis]....It is difficult to be specific or even to generalize about the accuracy of analytical results when there is a great variation in the type of samples analyzed and in the methods employed. A method which gives a certain accuracy on one type of material frequently does not give the same accuracy when applied to another type. Information on the mineralogical composition of the sample helps the chemists in the selection of methods of analysis and makes possible more and better analyses. The requester should give full details of any information he may have on the mineralogical and chemical makeup of his samples.
As an introduction to the topics covered in this publication and as a guide to alternative methods of analysis, the following table may prove useful. Listed for each element are estimates of sensitivity for the most common techniques used for their determination in geochemical samples. The term "sensitivity" is a general term for the lower limits of measurement for a given analytical test and is most often expressed as either a "detection limit" or "determination limit." The detection limit is the minimum concentration of analyte required for a positive decision that an analysis indicates a qualitative detection, and the determination limit is a higher level of concentration that will give a satisfactory quantitative result with a given relative standard deviation (such as \pm 10 percent). Both estimates are dependent on the analytical "noise" associated with measurement above the background or "blank" level for a given analytical procedure, and, for multielement analytical methods, the sensitivity limits are often matrix dependent and, therefore, may vary from sample to sample. The sensitivity levels provided by the table should be looked upon as "working" determination limits for a silicate rock matrix. As stated above, the method with the greatest sensitivity should not be identified as the "best" method for any given problem. The most sensitive methods are most often the most labor intensive. The method of choice is based on the answers to the following questions: What minimum levels of precision, accuracy, and sensitivity are required to solve the problem at hand? is a broad spectrum, multielement characterization required? and what interferences may preclude the analysis of a particular matrix by a given technique? Each of these questions should be answered by close collaboration between geologist and analyst at the onset of each geochemical study.

\section{ACKNOWLEDGMENTS}

Each chapter in this volume has benefited from review by U.S. Geological Survey scientists. The following individuals have contributed thoughtful reviews to one (or in some cases two) of the chapters: Joseph G. Arth, Robert A. Ayuso, Charles R. Bacon, Michael H. Bothner, David A. Brew, David A. Clague, Gerald K. Czamanski, Walter E. Dean, Franklin C.W. Dodge, Bruce R. Doe, Michael P. Foose, Carter B. Hearn, Rosalind T. Helz, Edward W. Hildreth, Joel S. Leventhal, Stephen D. Ludington, Frank T. Manheim, Andrei Sarna-Wojcicki, John Stuckless, and Howard E. Taylor. I wish to thank Janet Sachs for her editorial assistance. I also wish to thank Mary Catherine Kiel and Carol A. Popish for their clerical assistance. 


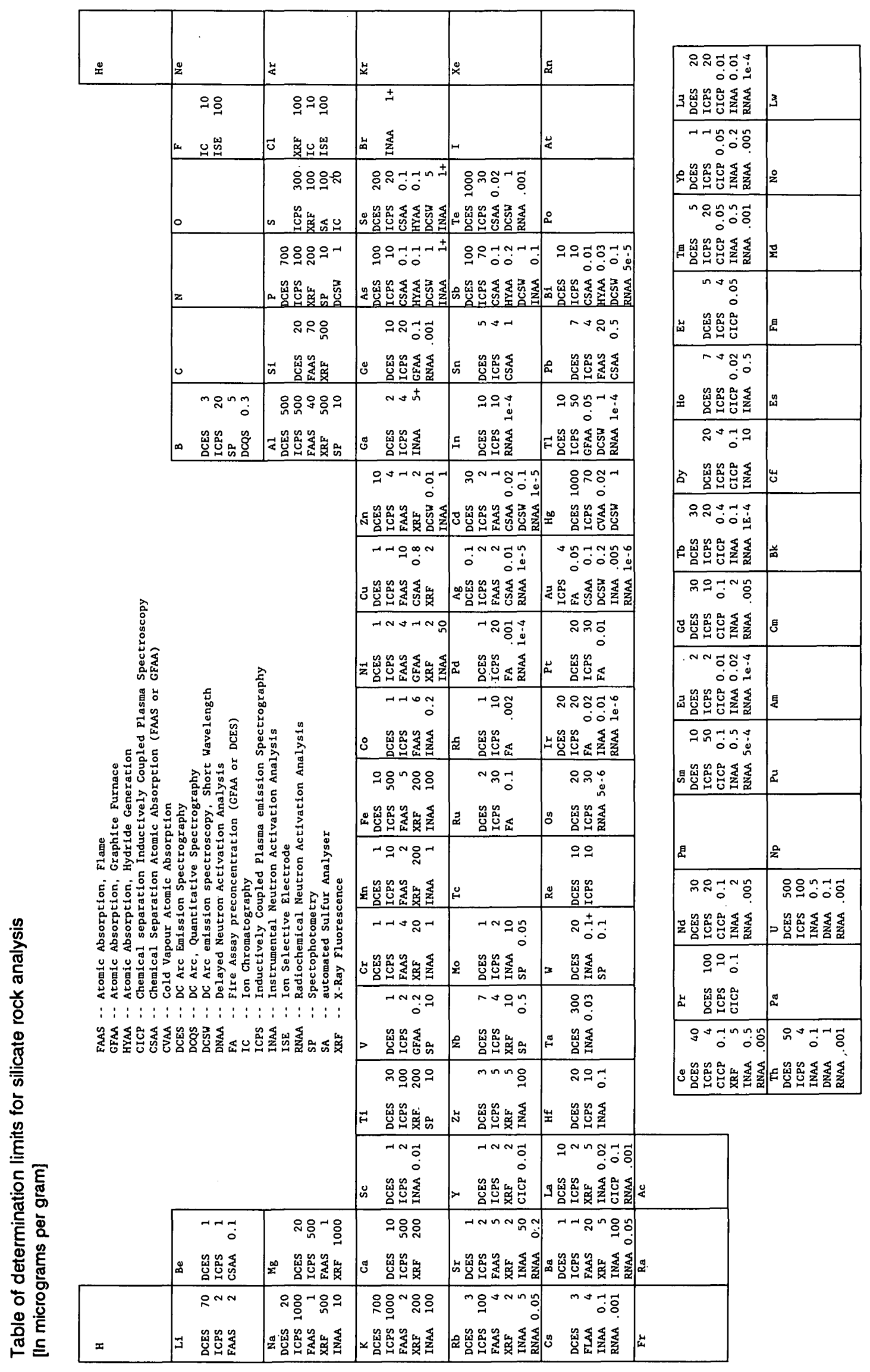



CHAPTER A

Analysis of Geologic Materials by Direct-Current Arc Emission Spectrography and Spectrometry

By D. W. GOLIGHTLY, A. F. DORRZAPF, JR., R. E. MAYS, T. L. FRIES, and N. M. CONKLIN

U.S. GEOLOGICAL SURVEY BULLETIN 1770

Methods for Geochemical Analysis 



\title{
CONTENTS
}

\author{
Abstract A1 \\ Introduction $\mathbf{A l}$ \\ General capabilities A1 \\ Basis of technique A1 \\ Experimental A2 \\ General A2 \\ Sample preparation and handling $\mathbf{A 3}$ \\ Standards A4 \\ Control of direct-current arc plasmas \\ Atmospheres A4 \\ Buffers A4 \\ Instrumentation and facilities $\mathbf{A 5}$ \\ Data collection A10 \\ Accuracy and precision of analysis A11 \\ Conclusions All \\ References cited $\mathbf{A 1 2}$

\section{FIGURES}

1. Vertical direct-current arc (idealized) A2

2. Generalized arrangement of components for direct-current arc spectrography

A3

\section{TABLES}

1. Semiquantitative analysis by direct-current arc spectrography: Elements and lower determination limits A6

2. Quantitative analysis by direct-current arc spectrography and direct-reading spectrometry: Elements and lower determination limits $\mathbf{A 8}$

3. Quantitative analysis by direct-current arc spectrography of some chalcophiles plus gold and phosphorus: Elements and lower determination limits A10

4. Generalized groupings of elements according to lower determination limits by quantitative direct-current arc spectrography and spectrometry $\quad \mathbf{A 1 2}$ 



\title{
Analysis of Geologic Materials by Direct-Current Arc Emission Spectrography and Spectrometry
}

\author{
By D. W. Golightly, A. F. Dorrzapf, Jr., R. E. Mays, T. L. Fries, and N. M. Conklin
}

\section{Abstract}

Direct analysis of diverse geologic materials for more than 68 elements occurring at trace and subtrace concentrations is achieved by direct-current arc spectrometric methods. The basis and capabilities of the methods currently used in the analysis of geological samples at the U.S. Geological Sunvey are described. The lower limits of determination for quantitative and semiquantitative methods are listed for all elements now determined by direct-current arc spectrography and spectrometry.

\section{INTRODUCTION}

The direct-current (d-c) arc is one of several electrical discharges used as light-emitting sources in analytical atomic spectroscopy. The high-temperature, radiating, gaseous volume between the two electrodes that determine the position of an arc in space is termed a laboratory "plasma." This plasma is a very high temperature gas $(5,000-7,000 \mathrm{~K}$, in the core) that is capable of atomizing, ionizing, and exciting quantized emissions (photons) from most elements of the periodic system. Spectral measurements on light emitted from a d-c arc into which a natural or fabricated material is vaporized provide the basis for determining the concentrations of up to 68 elements at trace levels.

This chapter provides a brief overview of some of the characteristics and capabilities of d-c arc spectrography (photographic plate detection) and spectrometry (electrical detection). Comprehensive treatments of this subject can be found in Boumans (1966) and Ahrens and Taylor (1961).

\section{General Capabilities}

In general, low part-per-million (micrograms of element per gram of sample) concentrations of most of the naturally occurring elements can be measured for arced samples ranging in mass from 10 to $20 \mathrm{mg}$. Only solid-phase samples can be analyzed conveniently by this technique, and these samples typically are finely pulverized ( -100 to -200 mesh) rocks, minerals, soils, residues, ferromanganese crusts and nodules, ashes from plants, peats, and coals, whole coals, ceramics, or metals.

All elemental analyses by this method are based on comparisons of measured spectral signals between samples and standards. Thus, good, naturally occurring, and synthesized reference materials are essential to highquality analyses. Quantitative analyses that are accurate to within \pm 10 percent can be achieved routinely by available methods for up to 55 elements. Semiquantitative analyses for up to 64 elements can be done with a typical uncertainty of -33 and +50 percent.

\section{Basis of Technique}

Emission of electromagnetic radiation from free atoms and ions is quantized, in accordance with the Bohr frequency condition: $\Delta \mathrm{E}=\mathrm{h} \nu$, where $\Delta \mathrm{E}$ is the photon energy, $h$ is Planck's constant, and $\nu$ is the difference in energy between two electron energy states for which a transition is allowed for a particular atom or ion. These energy differences between states are less than $15 \mathrm{eV}$ for typical spectral measurements made in the ultraviolet (UV) to visible range of frequencies (or wavelengths).

The spontaneous transition of an electron from an upper energy state, $q$ (excited state), to a lower energy state, $p$, is associated with the emission of a photon. The population of atomic energy states, or levels, is a thermal process that has been described by Boltzmann. Moreover, the energy levels of a particular atom or ion do not have equal probabilities of being populated by the bound electrons of an atom. Thus, thermal and statisticalmechanical considerations have given rise to the EinsteinBoltzmann equation (Boumans, 1966), which describes the intensity of a spectral line as a function of temperature, free-atom density, and other basic atomic characteristics,

$$
I=(d / 4 \pi) h v_{q p} A_{q p}\left(g_{q} / Z\right) n e^{-E_{q} / k T},
$$


where

I = spectral line intensity (watts per square centimeter per second per nanometer per steradian),

$\pi=3.14159$

$d=$ depth of the emitting source (arc),

$\mathrm{h}=$ Planck's constant,

$\nu=$ frequency of emitted photon,

$A_{q p}=$ Einstein transition probability for spontaneous emission,

$\mathrm{g}_{\mathrm{q}}=$ statistical weight of the upper energy level,

$\mathbf{Z}=$ partition function for particular atom or ion species,

$\mathbf{n}=$ number density of free atoms or ions of the species of interest,

$E_{\mathrm{q}}=$ energy of the upper level for transition,

$\mathbf{k}=$ Boltzmann's constant, and

$\mathrm{T}=$ absolute temperature (kelvins).

Both $\mathbf{n}$ and $\mathbf{Z}$ are functions of temperature. For a given species in the arc discharge column, $n$ is dependent on thermal and kinetic processes. The thermodynamic processes are described sufficiently by the Saha-Eggert equation for ionization and by Gibbs free-energy-based, equilibrium constant-temperature relations for dissociation of molecular species. The existence of local thermal equilibrium in the $\mathrm{d}-\mathrm{c}$ arc makes possible an approximate mathematical model of this emission source. The nonisothermal character of the arc discharge column can be accounted for in spectral measurements of temperature and electron density (Golightly and others, 1977) through recognition of the cylindrical symmetry of the arc and the subsequent use of the Abel transformation. Although this thermal model appears to be quite adequate, no similar model for kinetic processes exists to describe the vaporization or sublimation of materials from the anode (sample containing electrode) into the arc or the transport of material through the gap between the electrodes. Thus, no adequate "fundamental parameter" approach currently exists for correcting spectral line intensities analogous to that used in X-ray fluorescence spectrometry.

Quite importantly, the lack of a complete model based on theoretical considerations does not detract from the usefulness of the $d-c$ arc source in the trace analysis of geologic materials. A large body of published methodology exists to guide the analyst through very diverse applications of this well-established technique [for example, see Ahrens and Taylor (1961)].

\section{EXPERIMENTAL}

\section{General}

Implicit in the previous discussion is an understanding that the d-c arc is generated between two vertically coaxial cylindrical electrodes, as shown in figure 1 . The lower electrode (anode) invariably is $6-7 \mathrm{~mm}$ in diameter and has a cup-shaped tip into which the sample, frequently mixed with other materials, is tamped. The cathode is a thin (3-mm-diameter), pointed rod that is separated from the anode by a gap of a few millimeters ( $4 \mathrm{~mm}$ is typical). Both electrodes are composed of high-purity graphite for most applications. In addition to the feature of purity for graphite, for machined electrodes, and for graphite powder added to samples for the purpose of controlling volatilization rates, graphite has excellent electrical and thermal conductivity and sublimes, rather than melts, at the high temperatures created by arcing in an electrode cup $(\sim 3,000 \mathrm{~K})$.

The temperature and electron density in the arc plasma are effected by the power density (watts per cubic centimeter ) dissipated within the arc gap; that is, the space between the electrode tips. For a given, constant current (typically 10-30 A) across the arc gap, the power dissipated by Joule heating (typically $250-400 \mathrm{~W}$ ) is dependent wholly on the electrical resistivity of the gap$P=I^{2} R$, where $P=$ power (watts), $I=$ current (amperes), and $R=$ resistance (ohms). Thus, as the graphite electrode tips vaporize or burn away, adjustments of the arc gap must be made on a continuous basis to maintain a constant arc gap and, thereby, to provide stable, reproducible, power dissipation. Furthermore, the conductivity of the arc gap is influenced greatly by the materials (sample, graphite, chemical buffers, and so forth) that are transported through the arc gap during the arcing process. Lack of control of the gap distance and of the processes related to the transport and dissociation of materials in the gap frequently limits the precision and accuracy of analysis by this technique.

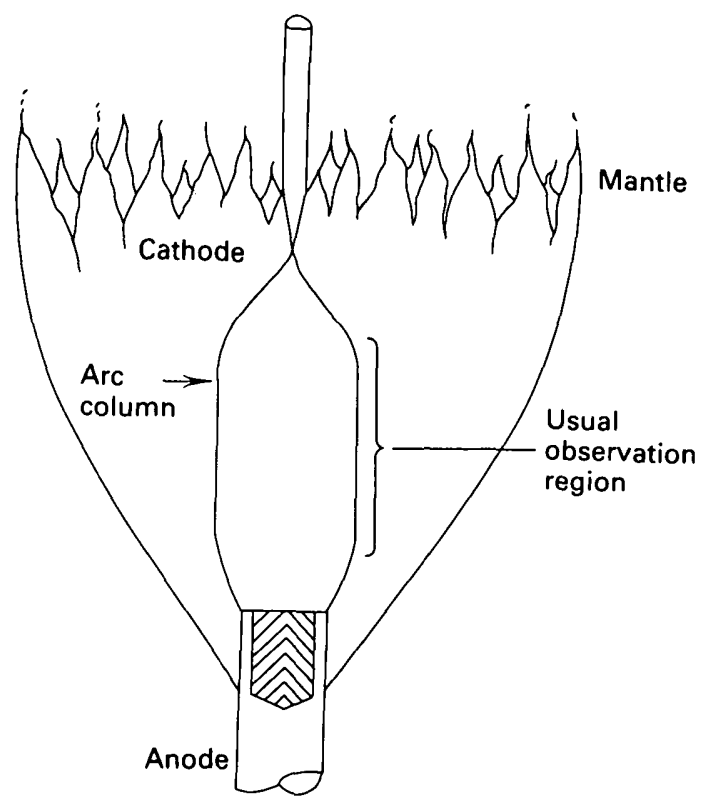

Figure 1. Vertical direct-current arc (idealized). 
Spectral dispersion of optical radiation from the arc usually is accomplished by spectrographs or by spectrometers that rely on diffraction gratings (fig. 2). Only a few prism instruments (that is, spectrographs that are based on the refraction of light) are available in our laboratories. Individual gratings are blazed to give maximal reflection in a specified region of the UV or visible part of the spectrum. The blaze angle of an engine-ruled grating refers to the angle at which the stylus of a ruling engine cuts the grooves into the metallic surface layer of the blank of an original grating. The blaze angle determines the spectral region into which the maximal reflection of diffracted radiation occurs. For the most part, replicate gratings, rather than original, engine-ruled gratings, are used in the Geological Survey's spectrographic instrumentation. Also, a few gratings produced by laser holographic processes now are in use. These holographic gratings are blazed, free of ruling irregularities that produce "spectral ghosts," and produce very low levels of scattered light. Conventional optical mounting configurations, such as the Ebert, Eagle, or Wadsworth mounts, are incorporated into the designs of the spectrographs in our laboratories. For direct-reading spectrometers (that is, instruments having photoelectric detectors), Rowland circle-based mounts, such as the Paschen-Runge, are used. The firstorder reciprocal linear dispersion of these spectrographs and spectrometers is approximately $0.5 \mathrm{~nm} / \mathrm{mm}$.

Maximal horizontal illumination of most of the grating surface is necessary for the highest possible spectral resolution from a given grating; that is, the resolving power (RP) of a grating is equal to the number of grooves illuminated, and, for the conventional spectrograph in our laboratories, the RP $=67,000$. This theoretical resolving power implies a resolution of $0.005 \mathrm{~nm}$ at $350 \mathrm{~nm}$ and is quite compatible with a first-order spectral band pass of $0.0125 \mathrm{~nm}$ for a $25-\mu \mathrm{m}$ entrance slit in a spectrographic mount that is likely to exhibit some optical aberration, such as coma, which is characteristic of the Ebert mount. The spectral band pass of a particular spectrograph and source line broadening (Doppler, pressure) determine the specificity of analysis. Although approximately 100,000 spectral lines have been identified in the 200- to 1,000-nm region (Meggers and others, 1975; Harrison, 1969), the specificity of emission spectrographic methods generally is quite good.

Uniform vertical illumination of the entrance slit is essential to spectrographic calibrations for the response of photographic emulsions. Such detailed calibrations are required because of the large variations in the rate of blackening of the emulsion as a function of wavelength and because only the photographic exposure, which is the flux density rate-time integral, can be related directly to spectral line intensities. These response calibrations typically depend on multiple-step neutral density filters or on step sectors. The accurately known ratios (usually ranging from 1.5 to 3 ) of the transmissions of steps of a filter or sector provide the basis for calibration of the photographic emulsion. Such a calibration is required to relate transmission, as measured by microphotometry, to exposure and, thus, to relative intensity.

Attenuation of light entering the spectrograph or spectrometer usually is effected by neutral density filters. Sorting of spectral orders (that is, diffraction orders that are part of the Bragg equation) is accomplished through the use of sharp-cut filters or of narrow-band pass filters, combined with an appropriate selection of photographic emulsions or of photocathodes for photomultipliers.

\section{Sample Preparation and Handling}

Solid samples generally are reduced to -100 mesh powders by the grinding laboratories. Typically, up to

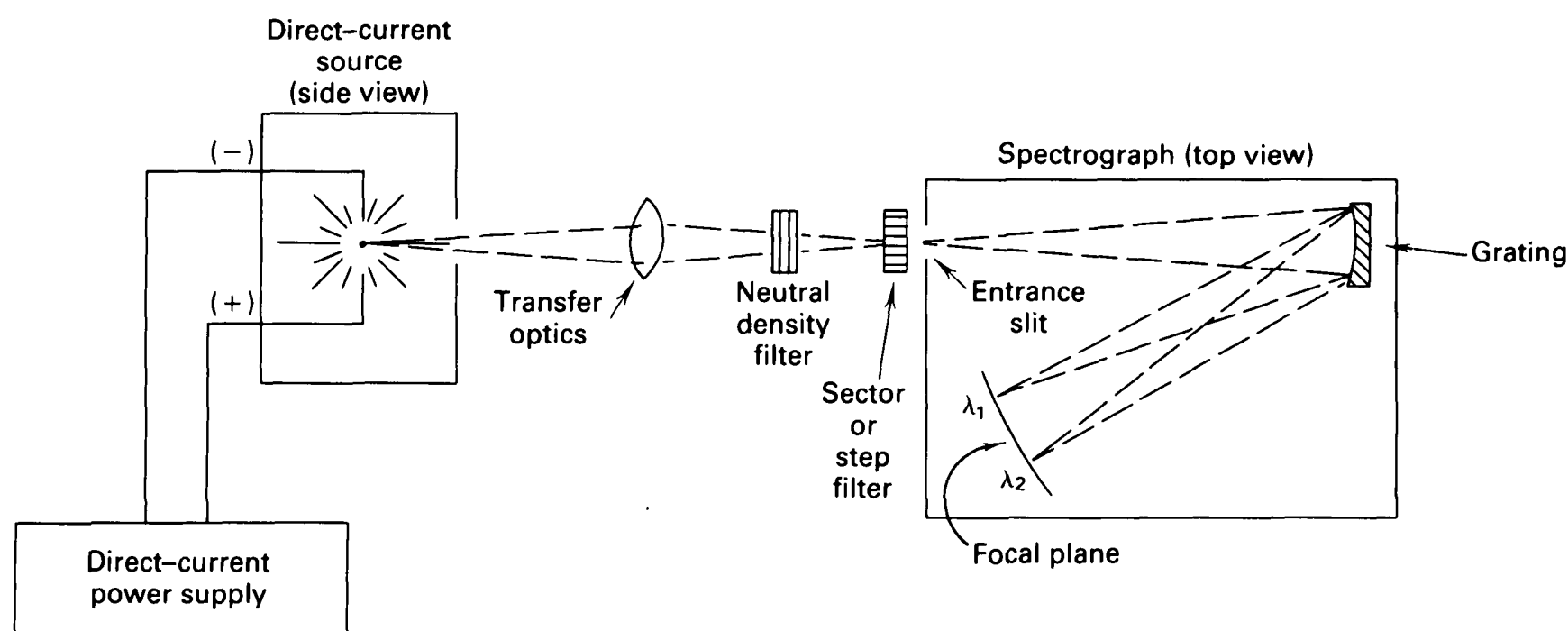

Figure 2. Generalized arrangement of components for direct-current arc spectrography. $\lambda_{1}$ and $\lambda_{2}$ indicate two different spectral lines. 
kilogram quantities of rock aggregates are passed through a manganese steel jaw crusher and finally are reduced to -100-mesh particle size in an alumina-plate vertical grinder. For quantitative analysis by $\mathrm{d}-\mathrm{c}$ arc, direct-reading spectrometry, samples are pulverized to -200 mesh in an agate puck and ring grinder. All sieving is done through nylon or stainless steel screens. Between-sample cleanup of active surfaces of grinding devices is accomplished by grinding clean quartz sand. The goal of the overall process is to reduce the aggregate material to small particles without contributing contaminants from the grinding surfaces or from sieves and to minimize the possibility of contaminating one sample with another (cross contamination). The thorough mixing of the generated particles that occurs in the grinding process is an additional benefit to the analyst who almost always needs only a small subsample of a homogeneous pulverized sample. In a few instances, especially when it is necessary to analyze a small $(<1-\mathrm{g})$ mineral phase, the analyst manually grinds the sample with an agate or boron carbide mortar and pestle.

Within the atomic emission spectrometry laboratories, a small quantity (100- to $200-\mathrm{mg}$ scale) of each pulverized sample received from the Grinding Laboratories is handled in accordance with the methodology established by chemists at a particular center (Annell, 1967; Bastron and others, 1960; Dorrzapf, 1973; Fletcher and Golightly, 1985; Haffty and others, 1977; Helz, 1973; Helz and others, 1969; Heropoulos and others, 1984; Mays, 1974; Myers and others, 1961; Thomas, 1979; Walthall, 1974). For purposes of semiquantitative analysis (Dorrzapf, 1973), $15 \mathrm{mg}$ of sample is mixed manually with $30 \mathrm{mg}$ of -200 mesh graphite in an aluminum pan, and the total $45 \mathrm{mg}$ of material is transferred quantitatively into the cup of a graphite electrode. In some procedures for quantitative analysis, sample is mixed with soda quartz (a mixture of sodium carbonate and quartz) by grinding the materials together in an agate mortar. Graphite, doped with an internal standard element, such as one of the less-abundant platinum metals, commonly is mixed with sample inside a closed, cylindrical polyethylene container that is rapidly agitated by a motor-driven device. Handling procedures are dictated largely by the analytical measurements needed and by the sample size. The reasons for mixing samples with graphite, soda quartz, or some other material are discussed in the section, "Buffers."

\section{Standards}

Commonly, well-characterized rocks and minerals are used from the large set of diverse reference materials known as International Standards (Flanagan, 1973, 1976). Because of the dynamic volatilization and excitation char- acteristics of the d-c arc, the use of standards that are similar to the samples to be analyzed is essential to accuracy. In cases where a similar standard does not exist or is unavailable, the method of standard additions provides a quite valid approach. However, the alternate approach usually taken involves the "synthesis" of standards by mixing high-purity oxides of sample-matrix elements with silica or the oxide of the principal matrix element. This approach to "synthetic" standards applies to silicate, carbonate, phosphate, sulfide (Mays, 1974), and all other mineral and rock types. Such synthetic standards frequently are used in calibrating "instrumental response," which, in turn, can be fitted to a few existing natural standards to provide accurate extrapolations of concentration over wide ranges of concentration.

\section{Control of Direct-Current Arc Plasmas}

\section{Atmospheres}

The gaseous atmosphere in which a d-c arc is operated greatly controls the transport of analytes, the emitting species present, and the excitation of analyte atoms and ions. Although arcs in ambient air still are used in many applications, distinct advantages have been established for argon and argon-oxygen atmospheres, which are in use at the three centers; for example, a 70-percent Ar-30-percent $\mathrm{O}_{2}$ mixture, used in the Reston semiquantitative analysis procedure (Dorrzapf, 1973) provides improved detectability for the lanthanide elements and, at the same time, gives a spectral background that is free of cyanogen bands; that is, the extensive spectral band structure from the $\mathrm{CN}$ radical. This species is generated by the reaction of nitrogen in air with the hot graphite of the anode. In addition, a 25- $\mathrm{A} \mathrm{d-c}$ arc in an argon atmosphere provides an atmosphere conducive to the measurement of $\mathrm{Li}, \mathrm{Rb}$, and $\mathrm{Cs}$ at 1-ppm concentration levels (Annell, 1967). In both cases, laminar flows of Ar- $\mathrm{O}_{2}$ and of $\mathrm{Ar}$ are introduced concentrically to the anode by a ceramic nozzle (Helz, 1964).

\section{Buffers}

The term "buffer" commonly is used to refer to any material added to the sample to control the rate of vaporization of the sample into the arc column, to alter the volatility of a selected element or of a particular group of analyte elements, or to effect desired ionization and excitation conditions within the discharge column. Practical examples of each type of buffer are provided by (1) graphite powder, which is used in most quantitative analyses to inhibit rapid volatilization of sample constituents into the arc column, (2) soda quartz powder, which is 
a mixture of sodium carbonate and quartz to control vaporization and ionization processes, and (3) copper hydroxyfluoride, which forms volatile boron trifluoride that can rapidly escape the molten sample bead, decompose to give free boron atoms, and undergo thermal excitation to provide boron atomic emission signals. Obviously, this volatilization reaction is not specific for boron because many other elements form volatile fluorides. The important aspect of this approach to determining boron concentrations is that boron, in the absence of some special volatilizer, readily forms a refractory carbide that does not distill easily from a graphite electrode. Thus, the rapid release of boron by compounds, such as copper hydroxyfluoride or polytetrafluoroethylene, greatly enhances the boron atomic line signals and, accordingly, enables the d-c arc technique to determine boron at a concentration of $1 \mathrm{ppm}$.

The $d-c$ arc is recognized widely as a distillation column (Boumans, 1966). Components of a complex, fused sample (melted in the electrode cup by the arc) vaporize into the arc at rates dependent on the species present in the melt and on the individual vapor pressure of each species. Analytical spectroscopists generally tend to group elements into two simple categories-volatile and involatile. However, in practice, the careful analyst establishes the time required for individual analyte elements to distill from the anode. In spectrography, this is done by a "moving plate" study, and, in direct-reading spectrometry, one can gate electronically the amplifier of the detector channel of each analyte element to obtain a profile of emission-signal intensity versus time. The goal of this type of measurement is to establish the exact interval for generating the atomic line signal of interest from the arc. Then, the buildup of background signal, coming from either spectral origins or the detector, can be minimized to give the best possible signal-to-background ratio. Thus, signal quality in spectrography can be optimized by terminating the exposure interval before the total sample vaporizes from the anode or, in direct-reading spectrometry, by on and off gating of the amplifier for a particular spectral line only for the interval during which the element generating the spectral line is vaporized from the anode. Both approaches are used routinely in our laboratories.

Although not frequently used, matrix alteration methods exist for fusing geologic samples with sodium borate and then grinding the fusion pellet and arcing the powder. This approach destroys many common mineral matrices, thereby forcing all samples and standards to melt and distill identically, and provides good control of arcing conditions. However, this method is labor intensive and effectively dilutes all analyte elements, some to concentrations below desired determination limits.

Because arc temperatures generally are conducive to the production and excitation of neutral atoms, ele- ments having low ionization potentials, such as the alkali metals, are added to affect the ionization equilibria $\left(M^{\circ}=\right.$ $\mathrm{M}^{+}+\mathrm{e}^{-}$) in the arc plasma. The readily ionized alkali metals, added in sufficient quantity, provide an increased electron density in the plasma to drive the equilibrium toward the desired neutral atom state. These ionization buffers are in common use and, in terms of conventional methodology, are best represented by cesium in the chalcophile element method and by sodium in the soda quartz used in determinations of trace elements in silicate rocks and minerals. In addition to favorably affecting ionization equilibria, such buffers make the arc dynamics quite reproducible.

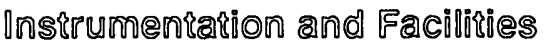

Each center has a complete spectrographic laboratory with the basic instrumentation to operate a d-c arc, to record spectra on photographic emulsions, to chemically process exposed emulsions, to do microphotometry of recorded spectral features (line peak transmittance, background transmittance, line width), and to relate these measured transmittances to relative exposures and, ultimately, to concentration. Although this general similarity among centers clearly exists, distinct dissimilarities are found in measurement capabilities that primarily originate from quite different instruments and from a different emphasis of methods that has developed partially from the partitioning of needs along regional geology lines. Some of these dissimilarities are best described by examples.

Currently, the Menlo Park laboratory has the only routinely operated d-c arc direct-reading spectrometer system. This system is used for semiquancitative analysis (table 1) and for the quantitative analysis of silicates for 43 elements (table 2). In addition, the Menlo Park laboratory has a method available that requires a shortwavelength-region-response emulsion and a grating blazed for $250 \mathrm{~nm}$ for spectrographically determining 11 elements in sulfide minerals (table 3 ). The Reston laboratory uniquely offers spectrographic methods for determining 28 elements in whole coals and coal macerals (Fletcher and Golightly, 1985), plus a method for trace (<1-ppm) boron in silicates. Also, Reston offers an automated 64-element semiquantitative spectrographic analysis (table 1), based on a computer-interfaced scanning microphotometer, whereas the laboratory in Denver provides semiquantitative analyses (table 1) based on visual comparisons of spectral lines between samples and standards. The Denver, Menlo Park, and Reston laboratories provide a d-c arc spectrographic method capable of determining cesium, lithium, and rubidium to lower limits of approximately $1 \mathrm{ppm}$ (Annell, 1967).

The foregoing discussion is meant to be a "sampler" on the diverse capabilities within the Division based 
Table 1. Semiquantitative analysis by direct-current arc spectrography: Elements and lower determination limits

[In parts per million; ND, not determined]

\begin{tabular}{|c|c|c|c|c|}
\hline \multirow[t]{2}{*}{ Element } & \multirow{2}{*}{$\begin{array}{l}\text { Average } \\
\text { Abundance } \\
\text { in } \\
\text { crustal } \\
\text { rocks } \\
\text { (Mason, 1966) }\end{array}$} & \multicolumn{3}{|c|}{ Lower deternination linit } \\
\hline & & $\begin{array}{l}\text { Denver: } \\
\text { Visual } \\
\text { (up to } 68 \\
\text { elesents) }\end{array}$ & $\begin{array}{l}\text { Reston: } \\
\text { Automated } \\
\text { scanning } \\
\text { micro- } \\
\text { photometer } \\
\text { (64 Elements) }\end{array}$ & $\begin{array}{l}\text { Menio Park: } \\
\text { Oịrect- } \\
\text { reading } \\
\text { spectrometer } \\
\text { (43 Elements) }\end{array}$ \\
\hline Ag & 0.07 & 0.5 & 0.1 & 1 \\
\hline A) & 81,300 & $100^{\mathrm{a}}$ & 460 & 2.500 \\
\hline As & 1.8 & 1.000 & 100 & 200 \\
\hline Au & 0.004 & 20 & 7 & 10 \\
\hline B & 10 & 20 & 3 & 10 \\
\hline Ba & 425 & 2 & 1 & 20 \\
\hline Be & 2.8 & 1 & 1 & 1 \\
\hline Bi & 0.2 & 10 & 10 & 10 \\
\hline Ca & 36,300 & 20 & 10 & 500 \\
\hline cd & 0.2 & 50 & 30 & 2 \\
\hline $\mathrm{Ce}$ & 60 & 200 & 40 & 100 \\
\hline co & 25 & 3 & 1 & 1 \\
\hline$c_{r}$ & 100 & 1 & 1 & 10 \\
\hline cs & 3 & $3^{b}$ & NO & MD \\
\hline cu & 55 & 1 & 1 & 1 \\
\hline Dy & 3 & $50^{c}$ & 20 & MD \\
\hline Er & 2.8 & $50^{c}$ & 5 & ND \\
\hline Eu & 1.2 & $100^{\circ}$ & 2 & ND \\
\hline Fe & 50.000 & 10 & 70 & 500 \\
\hline $\mathbf{G a}$ & 15 & 5 & 1 & 10 \\
\hline Gd & 5.4 & $50^{c}$ & 30 & NO \\
\hline Ge & 1.5 & 10 & 5 & ND \\
\hline HF & 3 & 100 & 15 & NO \\
\hline $\mathrm{Hg}$ & 0.08 & $1.000^{b}$ & ND & 500 \\
\hline Ho & 1.2 & $20^{c}$ & 7 & ND \\
\hline In & 0.1 & 10 & 10 & ND \\
\hline Ir & 0.001 & $50^{b}$ & 15 & NO \\
\hline$K$ & 25,900 & 7,000 & 680 & 800 \\
\hline La & 30 & 30 & 10 & 20 \\
\hline LI & 20. & 100 & 70 & 50 \\
\hline Lu & 0.5 & $30^{c}$ & 15 & ND \\
\hline Mg & 20,900 & 20 & 20 & 1,000 \\
\hline$M n$ & 950 & 1 & 1 & 200 \\
\hline Mo & 1.5 & 3 & 1 & 10 \\
\hline $\mathrm{Ma}$ & 28,300 & 500 & 20 & 1,500 \\
\hline
\end{tabular}


Table 1. Semiquantitative analysis by direct-current arc spectrography: Elements and lower determination limits-Continued

\begin{tabular}{|c|c|c|c|c|}
\hline \multirow[t]{2}{*}{ Element } & \multirow{2}{*}{$\begin{array}{l}\text { Average } \\
\text { Abundance } \\
\text { in } \\
\text { crustal } \\
\text { rocks } \\
\text { (Mason, 1966) }\end{array}$} & \multicolumn{3}{|c|}{ Lower deternination linit } \\
\hline & & $\begin{array}{l}\text { Denver: } \\
\text { Visual } \\
\text { (up to } 68 \\
\text { elewents) }\end{array}$ & $\begin{array}{l}\text { Reston: } \\
\text { Autoaated } \\
\text { scanning } \\
\text { oicro- } \\
\text { photometer } \\
\text { (64 Elements) }\end{array}$ & $\begin{array}{l}\text { Menlo Park: } \\
\text { Direct- } \\
\text { reading } \\
\text { spectrometer } \\
\text { (43 Eleaents) }\end{array}$ \\
\hline Nb & 20 & 10 & 7 & 25 \\
\hline Nd & 28 & $70^{d}$ & 30 & NO \\
\hline Mi & 75 & 3 & 1 & 1 \\
\hline os & 0.005 & $50^{6}$ & 15 & no \\
\hline $\mathbf{P}$ & 1,050 & 2,000 & 680 & 200 \\
\hline Pb & 13 & 10 & 7 & 10 \\
\hline Pd & 0.01 & 2 & 1 & ND \\
\hline $\operatorname{Pr}$ & 8.2 & $10^{\circ}$ & 100 & ND \\
\hline Pt & 0.01 & 50 & 2 & ND \\
\hline Rb & 90 & $3^{b}$ & No & NO \\
\hline Re & 0.001 & 50 & 10 & 50 \\
\hline Ph & 0.005 & $2^{b}$ & 2 & HD \\
\hline Ru & 0.01 & $10^{b}$ & 2 & no \\
\hline Sb & 0.2 & 150 & 70 & 100 \\
\hline se & 22 & 5 & 1 & 10 \\
\hline se & 0.05 & ND & ND & 200 \\
\hline Si & 277.200 & 20 & 50 & 100.000 \\
\hline Sa & 6 & $100^{d}$ & 10. & ND \\
\hline Sn & 2 & 10 & 5 & 10 \\
\hline$S r$ & 375 & 5 & 1 & 15 \\
\hline Ta & 2 & 500 & 320 & ND \\
\hline Tb & 0.9 & $300^{c}$ & 30 & No \\
\hline Te & 0.01 & 2.000 & ND & 50 \\
\hline Th & 7.2 & 200 & 50 & ND \\
\hline$T 1$ & 4.400 & 2 & 30 & 200 \\
\hline$T 1$ & 0.5 & 50 & 10 & 10 \\
\hline Ta & 0.5 & $20^{c}$ & 5 & NO \\
\hline $\mathbf{U}$ & 1.8 & 500 & 220 & ND \\
\hline$v$ & 135 & 7 & 1 & 10 \\
\hline$W$ & 1.5 & 100 & 15 & 100 \\
\hline $\boldsymbol{Y}$ & 33 & 10 & 1 & 10 \\
\hline$r_{b}$ & 3.4 & 1 & 0.2 & KD \\
\hline $2 n$ & 70 & 300 & 10 & 50 \\
\hline $2 r$ & 165 & 10 & 3 & 20 \\
\hline
\end{tabular}

al values below 0.1 percent ady result from grinding of sample with high-alunina ceranic plates.

Element deterained only if specifically requested.

Element determined only if $Y$ concentration is $>50 \mathrm{ppa}$.

dElement deternined only if $\mathrm{Ce}$ or $\mathrm{La}$ is found. 
Table 2. Quantitative analysis by direct-current arc spectrography and direct-reading spectrometry: Elements and lower determination limits [In parts per million; ND, not determined]

\begin{tabular}{|c|c|c|c|c|}
\hline \multirow[t]{3}{*}{ Element } & \multicolumn{4}{|c|}{ Lower deternination linit } \\
\hline & \multirow{2}{*}{$\begin{array}{l}\begin{array}{l}\text { Direct-reading } \\
\text { spectronetry }\end{array} \\
\text { Menlo Park }\end{array}$} & \multicolumn{3}{|c|}{ Spectrography } \\
\hline & & Reston & Denver & Menlo Park \\
\hline Ag & 1 & 1 & 0.5 & 0.2 \\
\hline Al & 2,500 & 20 & ND & No \\
\hline As & MD & 2,000 & MD & 150 \\
\hline Aus & ND & 40 & ND & 7 \\
\hline B & 10 & 20 & 20 & 2 \\
\hline Ba & 20 & 20 & 5 & 1 \\
\hline Be & 1 & 2 & 1 & 0.7 \\
\hline 81 & NO & 20 & MD & 7 \\
\hline Ca & 500 & 20 & ND & NO \\
\hline cd & 2 & 100 & 50 & 7 \\
\hline Ce & 100 & 200 & ND & 25 \\
\hline co & 1 & 4 & 5 & 1 \\
\hline$C_{r}$ & 10 & 2 & 7 & 1 \\
\hline$c_{s}$ & ND & $100^{\mathrm{a}}$ & MD & ND \\
\hline Cu & 1 & 2 & 1 & 0.7 \\
\hline Dy & ND & 100 & MD & 20 \\
\hline Er & No & 20 & No & 30 \\
\hline Eu & ND & 100 & ND & 0.1 \\
\hline Fe & 500 & 20 & ND & NO \\
\hline 6 & 10 & 10 & 7 & 0.7 \\
\hline $6 d$ & ND & 60 & ND & 5 \\
\hline $6 e$ & ND & 20 & 100 & 7 \\
\hline $\mathrm{HF}$ & No & 100 & ND & 50 \\
\hline Ho & ND & $1^{a}$ & ND & 10 \\
\hline Ho & ND & 40 & ND & 5 \\
\hline In & ND & 20 & ND & 1 \\
\hline Ir & ND & 100 & ND & 15 \\
\hline$K$ & 800 & $10^{\mathrm{a}}$ & ND & ND \\
\hline La & 20 & 40 & 50 & 7 \\
\hline LI & 20 & $2^{a}$ & 100 & 100 \\
\hline Lu & ND & 20 & ND & 15 \\
\hline Mg & 1,000 & 10 & Ho & ND \\
\hline$M_{n}$ & 200 & 2 & 2 & 0.7 \\
\hline Mo & 10 & 4 & 5 & 2 \\
\hline $\mathrm{Na}$ & 1,500 & $2^{a}$ & NO & MD \\
\hline
\end{tabular}


Table 2. Quantitative analysis by direct-current arc spectrography and direct-reading spectrometry: Elements and lower determination limitsContinued

\begin{tabular}{|c|c|c|c|c|}
\hline \multirow[t]{3}{*}{ Elewent } & \multicolumn{4}{|c|}{ Lower deteroination liait } \\
\hline & \multirow{2}{*}{$\begin{array}{c}\begin{array}{c}\text { Direct-reading } \\
\text { spoctrometry }\end{array} \\
\text { Menlo Park }\end{array}$} & \multicolumn{3}{|c|}{ Spectrography } \\
\hline & & Reston & Denver & Menlo Park \\
\hline Kb & 25 & 40 & 15 & 10 \\
\hline Nd & ND & 200 & No & 20 \\
\hline Mi & 1 & 4 & 5 & 0.7 \\
\hline os & NO & 100 & no & 30 \\
\hline $\mathbf{p}$ & 200 & 4000 & No & no \\
\hline Pb & 10 & 20 & 15 & 7 \\
\hline Pd & No & 6 & No & 1 \\
\hline Pr & No & 100 & No & 20 \\
\hline Pt & MD & 80 & no & 5 \\
\hline Rb & ND & $20^{\circ}$ & No & ND \\
\hline $\operatorname{Re}$ & ND & 20 & ND & 7 \\
\hline Rh & NO & 6 & NO & 3 \\
\hline Ru & No & $20^{a}$ & NO & 30 \\
\hline Sb & No & 200 & ND & 20 \\
\hline sc & 10 & 4 & 7 & 0.7 \\
\hline Si & 100,000 & 20 & ND & ND \\
\hline Sa & ND & 400 & no & 50 \\
\hline Sn & 10 & 20 & 10 & 2 \\
\hline sr & 15 & 4 & 7 & 1 \\
\hline Ta & ND & 400 & NO & 50 \\
\hline Tb & NO & 100 & NO & 100 \\
\hline Te & NO & 2,000 & NO & 300 \\
\hline Th & No & 1,000 & ND & 150 \\
\hline Ti & 200 & 10 & 10 & ND \\
\hline TI & ND & 100 & ND & 3 \\
\hline To & ND & 20 & ND & 2 \\
\hline$u$ & ND & 1.000 & No & 150 \\
\hline$v$ & 10 & 10 & 10 & 1 \\
\hline$W$ & ND & 400 & ND & 10 \\
\hline$Y$ & 10 & 20 & 10 & 7 \\
\hline rb & NO & 2 & 2 & 0.7 \\
\hline $2 n$ & 50 & 600 & 300 & 15 \\
\hline $2 r$ & 20 & 20 & 10 & 3 \\
\hline
\end{tabular}

A special exposure is required to deternine these elements. 
Table 3. Quantitative analysis by directcurrent arc spectrography of some chalcophiles plus gold and phosphorus: Elements and lower determination limits [In parts per million. Menlo Park SWR* method]

Elewent Lower determination linit

\begin{tabular}{lc}
\hline As & 1 \\
Au & 0.2 \\
81 & 0.1 \\
Cd & 0.1 \\
& \\
Hy & 1 \\
Sb & 1 \\
Se & 5 \\
T1 & 1 \\
Zn & 1 \\
P & 0.01 \\
\hline Sw refers to the ealsion type \\
\hline
\end{tabular}

on the existing facilities for d-c arc spectrography and spectrometry. Obviously, the technique and facilities can be molded to fit quite different information requirements on geologic materials.

\section{Data Collection}

Relative spectral line intensities, rather than absolute line intensities previously described by the EinsteinBoltzmann equation (eq 1), can be used in all determinations of elemental composition. Thus, the data-collection process begins with the measurement of the relative intensity of an isolated spectral line and the subsequent establishment of a functional relation between the relative intensity and the concentration of the element that emits that line. This relation commonly is called the analytical function and typically consists of a least-squares regression line that is fitted to data on a concentration versus relative intensity (or concentration ratio versus intensity ratio) plot. The resulting regression curve is referred to as the analytical curve or working curve. The analytical curve is established by arcing standard reference materials or other well-characterized materials, because the subsequent extrapolations of elemental concentration require that this calibration curve be extremely well defined.

The proper axes and shape of the analytical curve are inferred by the Scheibe-Lomakin equation (Boumans, 1966), which empirically relates the content, G, of an element in the sample and the intensity, I, of a spectral line in the arc,

$$
\mathbf{I}=\mathbf{K} \cdot \mathbf{G}^{\mathrm{m}} .
$$

Thus, intensity is proportional to concentration, but deviations from the proportionality, caused by self absorption, are accounted for by the exponent $\mathrm{m}$. $\mathrm{K}$ and $\mathrm{m}$ are determined by the analyst. When photographic recording of spectra is used, the Scheibe-Lomakin equation is depicted in logarithmic form,

$$
\log \mathrm{I}=\mathrm{m} \cdot \log \mathrm{G}+\log \mathrm{K} .
$$

For direct-reading spectrometry, the signal measured in the data-collection process is simply the photocurrent from the anode of a photomultiplier detector. This photocurrent is directly proportional to the light flux passing through an exit slit of the spectrometer, and, thus, for a properly chosen slit width, the photocurrent represents the flux over the complete spectral profile of a given line. The anode currents from photomultipliers, which have inherent amplification factors of up to 1 million, typically range from a few nanoamperes to hundreds of microamperes. Most importantly, these currentgenerating transducers, when supplied with proper dynode voltages, give linear responses over five to six orders of magnitude of input flux. The small currents from each photomultiplier are integrated by individual operational amplifiers over selected time intervals, and the resulting voltage on the capacitor in the feedback loop of the operational amplifier is used as a direct measure of spectral line intensity. Interfacing of such detectoramplifier electronics to minicomputer systems, as is done in all modern direct-reading spectrometers, enables very rapid measurements and extrapolations of concentrations. Gating of the operational amplifiers accomplishes optimal signal-to-background ratios, and automatic corrections for spectral interferences are possible with the appropriate algorithms on the minicomputer.

The approach to data collection in spectrography is built around the photographic emulsion and its unique characteristics as a detector of optical radiation. An image on the photographic emulsion is attributable to the interaction of light flux with silver halide grains over an interval of time. Thus, the emulsion inherently integrates the flux over time to give the exposure, which becomes the measure of relative intensity for spectral lines. Unlike the photomultiplier, this detector has a dynamic range that usually is less than $\mathbf{1 0 0}$. However, the emulsion gives a practically permanent record that can be studied at will and that provides information on hundreds of analytically 
useful lines and on spectral line shapes and potential spectral interferences. The $\mathbb{R}$ eston automated microphotometer system used for collection of data from photoplates for semiquantitative analysis measures the intensities of 260 spectral lines for emulsion calibration and of 400 spectral lines for the determination of 64 elements. No existing direct-reading spectrometer system, including echelle spectrometers with vidicon detectors, makes use of this spectral information. This automated scanning microphotometer, which is capable of making highprecision measurements of spectral line positions (within $\pm 0.005 \mathrm{~nm}$ ) and of optical transmission ( \pm 0.1 percent), has been used to a limited extent for quantitative measurements. However, conventional practice for the collection of data for quantitative spectrographic measurements relies on use of microphotometers that are capable only of measuring transmissions of single spectral lines.

\section{ACCURACY AND PRECISION OF ANALYSIS}

The processes that control vaporization, transport, and excitation in the d-c arc are each dynamic and each dependent on physical and chemical parameters. The principal parameters and their effects on atomic line signals were discussed in the previous section. The degree to which an experimentalist controls these parameters and the integrity of the arced sample ultimately determine the accuracy and the precision of analyses by $d$-c arc spectrography or direct-reading spectrometry.

Obviously, the arced sample must be a representative subsample of the original material that was collected in the field. Thus, the very stringent requirement for sample homogeneity on the $10-\mathrm{mg}$ scale is placed on all materials analyzed by this method. Recall that the final amount of material arced typically is $10-20 \mathrm{mg}$ and that the sample received by the Grinding Laboratory can be more than $1 \mathrm{~kg}$. In turn, the approximate $1-\mathrm{kg}$ field sample may be intended to represent a major outcrop or geological structure that weighs many metric tons. A simple weighing or weight-loss error of $0.1 \mathrm{mg}$ in $10 \mathrm{mg}$ will produce a relative error of 1 percent. However, significant sample heterogeneity will produce unacceptable systematic errors.

Apart from the quite serious requirement for sample homogeneity, the previously discussed physical parameters, including the electrical current and voltage, the arc gap, the composition (graphite or amorphous carbon), shape, dimensions, and porosity of electrodes, the temperature and electron density in the arc, and the chemical parameters, including reaction products in the hightemperature graphite anode, volatilization rates of species in the anode, dissociation equilibria of molecular species in the arc plasma, ionization equilibria of atomic species in the arc plasma, and diffusion rates of the atomic or ionic species of interest, must all be reproduced on a sample-by-sample basis. For the arc, almost all these parameters are time dependent and thus, unlike a steadystate system, such as X-ray fluorescence spectrometry or metal-chelate solution spectrophotometry, must be controlled tightly during each arcing sequence. Under good circumstances, these physical and chemical parameters can be controlled to give measurement uncertainties of less than 5- to 10-percent relative error. Furthermore, the data collection process adds a 1- to 3-percent uncertainty for microphotometry or $<1$ percent for direct-reading spectrometry. Thus, for well-controlled experiments in the measurement of concentrations of trace elements occurring well above their detection limits, one is justified in expecting relative errors in the range of 5-15 percent.

The accuracy of concentration measurements is nearly totally dependent on the matching of samples with standards. This match ideally includes the sample matrix (chemical and modal composition) and the approximate trace-element composition. For common rock types, this ideal matching is possible because of the existence of many International Standards (Flanagan, 1973). However, previously discussed matrix modifications (diluents and buffers) and the standard addition method can become quite important to successful analyses. Under good conditions, systematic errors range from 5 to 20 percent. Many exceptions to this generalization occur because the actual accuracy of analysis varies from element to element, spectral line to spectral line, and matrix to matrix.

\section{CONCLUSIONS}

The d-c arc source in spectrographic analysis provides a means for the highly selective and sensitive determination of up to 68 elements at trace concentrations in geologic materials. Sample handling and the attendant possibility for contamination are minimized by direct analysis of solid phase samples.

Methods for semiquantitative $d-c$ arc spectrography enable the determination of alkali metals, alkaline earths, most of the transition elements, and one-half the lanthanide elements at and below crustal abundance levels (table 1). The quality of semiquantitative spectrographic data is maintained by arcing one or more standards from a group of 20 International Standards on each exposed photoplate as a "control sample"; that is, the concentrations of all measured elements must fall within defined tolerance limits before other analyses from spectra on the same photoplate are considered valid. For common aluminosilicate rocks, the semiquantitative analysis provides concentration data that agree well with those from independent analysis techniques. Regardless of the type of sample matrix, the semiquantitative analysis method provides an 
excellent first analysis for a general overview of the elements present and their approximate concentrations.

The same elements determinable by the semiquantitative analysis method also can be measured quantitatively (table 2). Quantitative methods tend to focus on specific groups of elements that either are chemically similar or emit spectra in wavelength regions that require either a common detector or some other specific feature of a spectrometer or of source operation. However, quantitative methods place greater constraints on spectral line selection and on calibration. Rigorous calibration for each element is required from spectra of standard materials with matrices similar to those of the samples. This requirement most often represents a limitation for a spectrographic method on the number of elements that can be determined for a particular type of sample material.

Quantitative methods are in use for the determination of alkali metals (Annell, 1967), for the alkaline earths and most of the transitional elements and lanthanides (Bastron and others, 1960), and for part-per-billion concentrations of the platinum metals platinum, palladium, and rhodium (Dorrzapf and Brown, 1970; Haffty and others, 1977). Although the platinum metal determinations require forms of fire-assay preconcentration (see Chapter D), the other quantitative methods directly use the pulverized sample in the arcing procedure. A bestcase example of the sensitivity of the method is found for the element silver, which can be determined quantitatively by vaporizing less than $1 \mathrm{ng}$ of silver into the arc plasma. Although the sensitivity of d-c arc spectrographic methods is generally good, the principal limitations of these methods arise from potential spectral interferences, the effects of the matrix composition and form on the transport of material through the discharge column, and the lack of sufficient control of the arc to provide precisions of measurement better than 5- to 20 -percent relative standard deviations.

A useful, but quite generalized, overview of the capabilities of direct (without chemical preconcentration) quantitative d-c arc spectrography and spectrometry is provided by table 4 . This tabulation enables one to quickly surmise which elements are determined most readily ( $\mathrm{Ag}$, $\mathrm{As}, \mathrm{Au}, \mathrm{Ba}, \mathrm{Be}, \mathrm{Bi}, \mathrm{Cd}, \mathrm{Eu}$, and so forth) and which elements are not capable of being determined at low concentrations by direct d-c arc methods (U, Th). Cautious restraint must be used in the interpretation of this table because it contains no information on the accuracy, precision, or sensitivity of measurements, each of which is affected significantly by the sample matrix and by potential spectral interferences. Successful handling of these "experimental" effects and of the data generated in spectral measurements is totally dependent on the laboratory skills and interpretative capabilities of the analyst.
Table 4. Generalized groupings of elements according to lower determination limits by quantitative direct-current arc spectrography and spectrometry

[Best determination limits from tables 2 and 3 ; in parts per million]

Concentration interval

\begin{tabular}{lll}
$0.1-1 \quad>1-10 \quad>10-100 \quad>100-1,000$ \\
\hline
\end{tabular}

\begin{tabular}{|c|c|c|c|}
\hline Ag & B & Al & Th \\
\hline As & Co & $\mathrm{Ca}$ & u \\
\hline $\mathrm{Au}$ & $\mathrm{Cr}$ & Ce & \\
\hline $8 a$ & $\mathrm{Cu}_{\mathrm{u}}$ & Cs & \\
\hline Be & Gd & Dy & \\
\hline Bi & Ho & Er & \\
\hline Cd & K & $\mathrm{Fe}$ & \\
\hline Eu & La & Ge & \\
\hline Gs & LI & Hf & \\
\hline $\mathrm{Hg}$ & $\mathrm{Mg}$ & Ir & \\
\hline In & Ho & Lu & \\
\hline $\mathrm{Mn}$ & $\mathrm{Na}$ & Nd & \\
\hline $\mathrm{NI}$ & Nb & Os & \\
\hline P & $\mathrm{Pb}$ & $\mathrm{Pr}$ & \\
\hline Sb & $P d$ & $\mathrm{Rb}$ & \\
\hline Sc & $\mathrm{Pt}$ & Ru & \\
\hline $\mathrm{Sr}$ & $\operatorname{Re}$ & Si & \\
\hline Te & Rh & Sin & \\
\hline II & Se & $\mathrm{Ta}$ & \\
\hline$v$ & Sn & Tb & \\
\hline Yb & $T H$ & Ta & \\
\hline \multirow[t]{3}{*}{$2 n$} & H & & \\
\hline & $Y$ & & \\
\hline & $2 r$ & & \\
\hline
\end{tabular}

\section{REFERENCES CITED}

Ahrens, L. H., and Taylor, S. R., 1961, Spectrochemical analysis-A treatise on the $d-c$ arc analysis of geological and related materials [2d ed.]: Reading, Massachusetts, Addison-Wesley Publishing, $454 \mathrm{p}$.

Annell, C. S., 1967, Spectrographic determination of volatile elements in silicates and carbonates of geologic interest using an argon d-c arc: U.S. Geological Survey Professional Paper 575-C, p. C132-C136.

Bastron, Harry, Barnett, Paul R., and Murata, K. J., 1960, Method for the quantitative spectrochemical analysis of rocks, minerals, ores, and other materials by a powder $d-c$ arc technique: U.S. Geological Survey Bulletin 1084-G, p. G1-G182.

Boumans, P. W. J. M., 1966, Theory of spectrochemica excitation: New York, Plenum Press, 383 p. 
Dorrzapf, A. F., Jr., 1973, Spectrochemical computer analyses, argon-oxygen d-c arc method for silicate rocks: U.S. Geological Survey Journal of Research, v. 1, no. 5, p. 559-562.

Dorrzapf, A. F., Jr., and Brown, F. W., 1970, Direct spectrographic analysis for platinum, palladium, and rhodium in gold beads from fire assay: Applied Spectroscopy, v. 24, p. 415-418.

Flanagan, F. J., 1973, 1972 values for international geochemical reference samples: Geochimica et Cosmochimica Acta, v. 37 , p. 1189-1200.

ed., 1976, Descriptions and analyses of eight new USGS rock standards: U.S. Geological Survey Professional Paper $840,192 \mathrm{p}$.

Fletcher, J. D., and Golightly, D. W., 1985, The determination of 28 elements in whole coal by direct-current arc spectrography: U.S. Geological Survey Open-File Report 85-204, $10 \mathrm{p}$.

Golightly, D. W., Dorrzapf, A. F., Jr., and Thomas, C. P., 1977, Sets of spectral lines for spectrographic thermometry and manometry in d-c arcs of geologic materials: Spectrochimica Acta, v. 32B, p. 313-325.

Haffty, Joseph, Riley, L. B., and Goss, W. D., 1977, A manual of fire assaying and determination of the noble metals in geologic materials: U.S. Geological Survey Bulletin 1445, $58 \mathrm{p}$.

Harrison, G. R., 1969, Wavelength tables: Cambridge, The Massachusetts Institute of Technology Press, 429 p.

Helz, A. W., 1964, A gas jet for d-c arc spectroscopy: U.S. Geological Survey Professional Paper 475-D, p. D176-D178.
-1973, Spectrochemical computer analysis-Instrumentation: U.S. Geological Survey Journal of Research, v. 1, no. 4, p. 475-482.

Helz, A. W., Walthall, F. G., and Berman, Sol, 1969, Computer analysis of photographed optical emission spectra: Applied Spectroscopy, v. 23 , no. 5 , p. 508-518.

Heropoulos, Chris, Seeley, J. L., and Radtke, A. S., 1984, Spectrographic determination of selenium in stibnite: Applied Spectroscopy, v. 38, no. 3, p. 451-454.

Mason, Brian, 1966, Principles of geochemistry [3d ed.]: New York, John Wiley, p. 45-46.

Mays, R. E., 1974, Synthetic sulfide standards for quantitative emission spectrographic analysis: Applied Spectroscopy, v. 28 , no. 5 , p. $462-465$.

Meggers, W. F., Corliss, C. H., and Scribner, B. F., 1975, Tables of spectral line intensities: Washington, D.C., U.S. Government Printing Office, National Bureau of Standards Monograph 145, 387 p.

Myers, A. T., Havens, R. G., and Dunton, P. J., 1961, A spectrochemical method for the semiquantitative analysis of rocks, minerals, and ores: U.S. Geological Survey Bulletin 1084-I, p. I1-I229.

Thomas, C. P., 1979, A minicomputer based emission spectrographic analysis system dependent on scanning microphotometry: Applied Spectroscopy, v. 33, no. 6, p. 604-612.

Walthall, F. G., 1974, Spectrochemical computer analysisProgram description: U.S. Geological Survey Journal of Research, v. 2, no. 1, p. 61-71. 


\section{CHAPTER B}

Inductively Coupled Plasma-Atomic Emission Spectrometry

By F. E. LICHTE, D. W. GOLIGHTLY, and P. J. LAMOTHE

U.S. GEOLOGICAL SURVEY BULLETIN 1770

Methods for Geochemical Analysis 


\title{
CONTENTS
}

\author{
Abstract B1 \\ Introduction B1 \\ Experimental $\mathbf{B 2}$ \\ Instrumentation $\mathbf{B 2}$ \\ Instrumental optimization $\mathbf{B 3}$ \\ Sample preparation $\mathbf{B 3}$ \\ Acid dissolution $\mathbf{B 3}$ \\ Fusion B4 \\ Water analysis B6 \\ Manganese nodules B6 \\ Chemical separation B7 \\ Selective extraction $\mathbf{B 8}$ \\ Capabilities and limitations $\mathbf{B 8}$ \\ References cited B9
}

\section{FIGURE}

1. Typical instrumental arrangement for inductively coupled plasma-atomic emission spectrometry B2

\section{TABLES}

1 Detection limits in a granite for elements normally reported using an acid digestion $\mathbf{B 4}$

2. Analysis of reference standards by inductively coupled plasma-atomic emission spectrometry B5

3. Concentrations determined by neutron activation analysis, spark-source mass spectrometry, and inductively coupled plasma-atomic emission spectrometry of U.S. Geological Survey manganese nodule standards A-1 and P-1 B7

4. Rare-earth analysis by inductively coupled plasma-atomic emission spectrometry of U.S. Geological Survey standard basalt BCR-1 B8 



\title{
Inductively Coupled Plasma-Atomic Emission Spectrometry
}

\author{
By F. E. Lichte, D. W. Golightly, and P. J. Lamothe
}

\begin{abstract}
Inductively coupled plasma-atomic emission spectrometry is a technique that is capable of determining most elements at trace (2-100 parts per million), minor (0.01-0.5 percent), and major (greater than 0.5 percent) concentrations. The sample first must be dissolved and presented to the instrument as a solution. Water solutions can be analyzed directly or after preconcentration. Up to 44 elements can be determined simultaneously as a semiquantitative analysis. Specific elements can be determined quantitatively with high precision and accuracy. The method has been applied to a wide variety of exploration and geochemical studies involving major, minor, and trace element analysis of water; fluid extracts; extractable pathfinder elements; plants; coal; shale; and rock and minerals.
\end{abstract}

\section{INTRODUCTION}

The inductively coupled plasma-atomic emission spectrometric (ICP-AES) technique is useful for the determination of a wide variety of elements in geologic materials. For many metals, the technique possesses adequate sensitivity for direct determination following an appropriate dissolution procedure. For other elements, preconcentration techniques can be used to extend the lower limits of detection. The power of the ICP-AES technique stems from the high excitation energy of the source, which allows groups of elements to be determined simultaneously when using a direct-reading polychromator.

The ICP-AES technique offers better precision than direct-current (d-c) arc methods, superior limits of detection for most elements, and faster rates of analysis for multiple-element requests than flame atomic absorption spectrometry. Thus, the technique has assumed much of the analytical workload previously accomplished by d-c arc emission spectrography and flame atomic absorption spectrometry.

A typical instrumental arrangement is shown in figure 1 . The sample is introduced into the plasma by nebulizing a solution containing the sample and by trans- porting the resulting aerosol to the plasma in the sample injector gas. The injector gas punctures the center of the bottom of the plasma, and the sample travels through the plasma, which desolvates, melts, vaporizes, atomizes, ionizes, and, finally, excites the outer-shell electrons of the free atoms and ions of the elements in the sample. The relaxation of an excited electron is accompanied with the emission of a photon of light. The energy of this photon is characteristic of the atomic energy level transition and, thus, is characteristic of the element.

The grating of the spectrometer disperses the photons according to their energy, or wavelength. The intensity of a selected discrete wavelength of light is measured by a photomultiplier tube placed behind an exit slit.

The intensity of radiation for spontaneous emission is described by the Einstein-Boltzmann equation;

$$
I_{q p}=\frac{d}{4 \pi} A_{q p} h v_{q p} n \frac{g_{q}}{Z} \mathrm{e}^{-\left(E_{q} / k T\right)} .
$$

This relation assumes the existence of local thermal equilibrium, and the variables are defined as follows:

$$
\begin{aligned}
& I_{q p}=\text { intensity of radiation, } \\
& \mathbf{k}=\text { Boltzmann's constant, } \\
& \mathbf{n}=\text { number of free atoms of species of interest, } \\
& T=\text { absolute temperature, } \\
& g_{q}=\text { statistical weight of upper energy level, } q, \\
& Z=\text { partition function, } \\
& E_{q}=\text { energy of upper level } q, \\
& d=\text { depth of the source, } \\
& A_{q p}=\text { transition probability for the generalized } \\
& \quad \text { decay from upper energy level, } q, \text { to lower } \\
& \quad \text { energy level } p, \\
& h= \text { Planck's constant, and } \\
& \nu=\text { Frequency of emitted photon for } q \text { to } p \\
& \text { transition. }
\end{aligned}
$$

The design of the instrument is optimized for the intensity of this light source. The transition probability, $A_{q p}$, is unique for each transition and varies greatly from one element to another. In addition, each element can have many such transitions; for instance, the alkali metals have fewer than 10 observable transitions at temperatures 


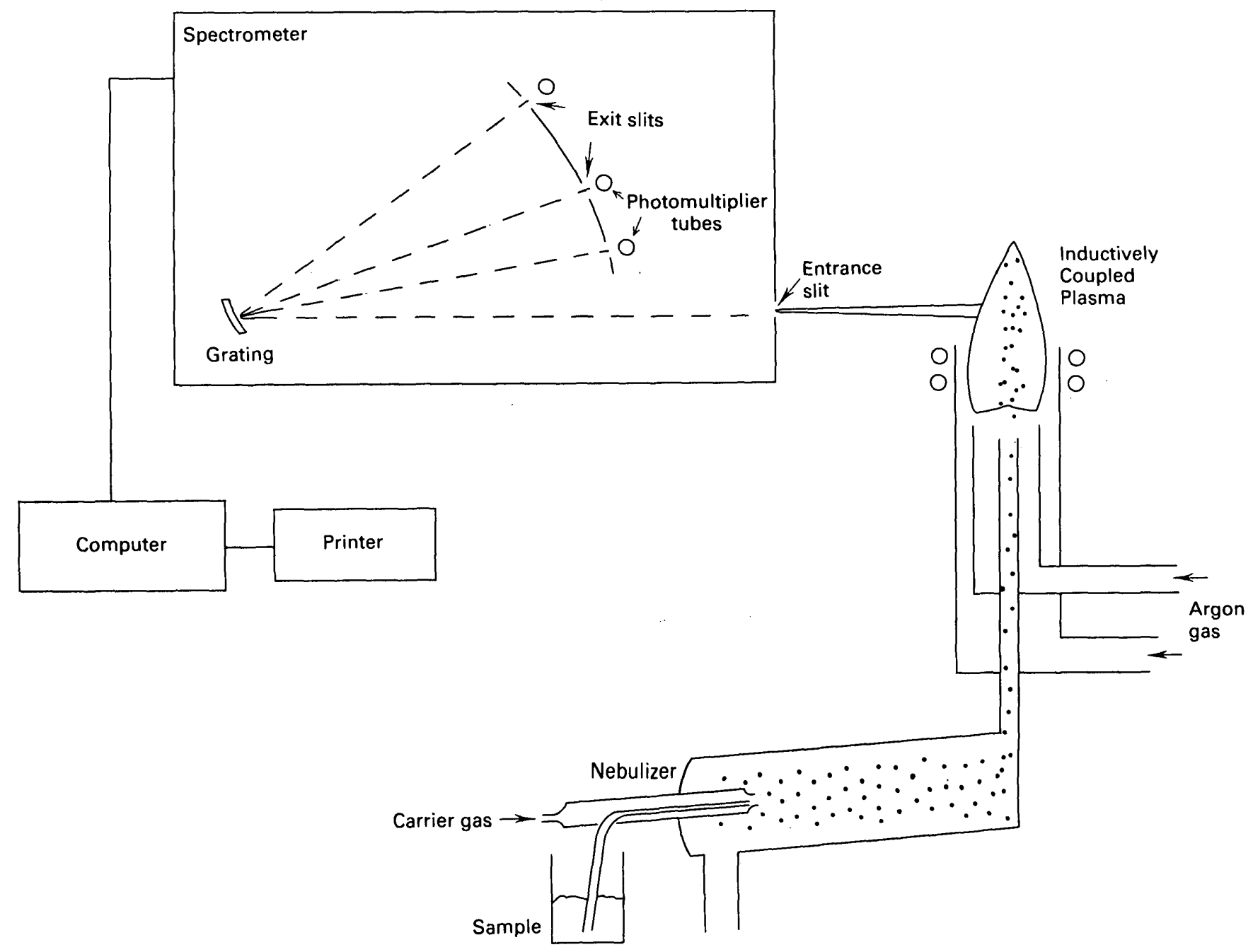

Figure 1. Typical instrumental arrangement for inductively coupled plasma-atomic emission spectrometry.

common to the ICP, whereas iron has more than 10,000 observable transitions.

Equation 1 indicates that, at a given temperature, the intensity of light emitted from an element in the plasma is directly proportional to the concentration of the element, n. One important feature of the ICP-AES technique is that the calibration curves are linear over a large range of concentrations. The linear working range for many colorimetric and atomic absorption spectrometric techniques is only two orders of magnitude of concentration, whereas the ICP-AES technique typically has a linear response of four to five orders of magnitude of concentration. The plasma consists of Ar ions and free electrons and produces excitation temperatures of approximately $6,000 \mathrm{~K}$, is very stable, and, coupled with its high excitation temperature, gives a very high signalto-noise ratio for spectral lines of over 50 elements.

The instrument is calibrated for the elements of interest by using specially prepared standards and (or) dissolved reference standards. A computer calculates the results, elemental concentrations, from these calibration curves.

\section{EXPERIMENTAL}

\section{Instrumentation}

The centers in Denver, Colorado, and Reston, Virginia, have virtually identical instrumentation. These instruments have approximately 60 fixed channels for measuring the emission from about 55 elements. Some elements require more than one channel to cover the wide dynamic range of concentrations found in geologic samples. The instruments were purchased at different times, and different priorities were used in selecting which elements and which wavelengths would offer the best results for the types of studies in each region. The complexity of describing line selection is beyond the scope of this chapter. However, criteria for line selection generally are based on the goals of minimizing spectral overlap 
and optimizing sensitivity for the most commonly analyzed matrix. Both instruments also have a scanning channel that enables any element to be included in the analysis program. This channel is set manually to the wavelength of interest.

The instrumentation in Menlo Park, California, is an automated scanning spectrometer. This instrument can analyze only one element at a time, but, because the instrument is under computer control, several elements can be analyzed for a given sample relatively easily. The flexibility for line selection offers an opportunity to analyze samples that contain highly variable concentrations of major elements with minimal spectral interferences. Because only one element can be analyzed at a time, fewer elements can be analyzed per unit time, and the precision is somewhat poorer than for the simultaneous instruments. Examples of the application of multichannel and single-channel instruments are discussed below.

\section{Instrumental Optimization}

As discussed above, the optics of the emission spectrometer are optimized for the photon flux of an argon plasma. The plasma itself is highly complex and deviates significantly from local thermodynamic equilibrium. The Boltzmann-Einstein equation (eq 1), thus can be applied only in well-defined cases. The plasma is also a spatially inhomogeneous excitation source, and the optimal temperature for a given transition, as defined in equation 1 , is found in a very small region of the plasma. Because only one region of the plasma is observed by the spectrometer, multielement determinations are made under compromise conditions. These conditions are determined by the specific requirements of the analytical program.

Three interrelated parameters of the plasma commonly are optimized for each program-radio frequency power, sample injector gas-flow rate, and observation zone of the plasma, defined as height above the load coil. Some general trends do exist for the optimization. Effective excitation of neutral atoms requires the low temperatures that occur by operating with low power, highinjection gas-flow rates, and high observation height. Transitions from ionic species require moderate power, lower injector gas-flow rates, and a moderate observation height (15 mm above the load coil). Analysis of samples containing high concentrations of refractory materials requires high input power, low carrier gas-flow rates, and a different plasma torch design that increases the length of the plasma to allow a long residence time of the analyte in the plasma. The observation height for this torch is high, even for ionic species.

Another parameter that is related to the plasma conditions is the droplet size distribution of the aerosol entering the plasma which plays a major role on the kinetics for the vaporization, desolvation, and dissociation processes of the analyte. The droplet size distribution is determined largely by the nebulizer and spray chamber. Most instruments use a concentric or cross-flow pneumatic nebulizer, which is appropriate for most applications. Solutions having a high salt content and (or) containing particulate matter require a Babington-type nebulizer to avoid plugging the small orifices used in the first two designs. A glass frit nebulizer can be used for samples of limited size, such as mineral separates. Ultrasonic nebulizers are useful in some applications. The choice of nebulizer is largely dependent on the type of dissolution procedure used in the preparation; for instance, chromium, when present in basalt, can be readily dissolved by using mineral acids. The resulting solution contains about 0.5 percent total salt, and the concentric or cross-flow nebulizer can be used. If the chromium is present in a mineral such as chromite, a fusion is required to dissolve the chromite. Because the resulting solution will contain 4 percent total salt, a Babington-type nebulizer may be more appropriate. The choice of nebulizer, torch, and plasma conditions must be selected and optimized around the dissolution procedure and the specific requirements of the analytical program. The next section deals with specific examples based on sample preparation procedures.

\section{Sample Preparation}

\section{Acid Dissolution}

Perhaps the most widely used method of dissolution is the acid digestion using hydrofluoric acid. The method used in Denver (Crock and others, 1983; Kane, 1985; Taggart and others, 1981) digests $0.2 \mathrm{~g}$ of -100 mesh sample by using appropriate amounts of $\mathrm{HF}$, aqua regia, and $\mathrm{HClO}_{4}$, and the resulting solution is taken to dryness on a hotplate. The salts are then dissolved in $1 \mathrm{~mL}$ of aqua regia and diluted to $10 \mathrm{~g}$ with 1 percent $\mathrm{HNO}_{3}$. Lutetium is used as an internal standard. This digestion vaporizes silicon and boron but is appropriate for 44 elements in silicate and carbonate rocks. The initial sample size and final volume can be adjusted to match specific sample requirements; for instance, a l-mg sample can be analyzed when dissolved in $2 \mathrm{~mL}$ of solution. Although the detection limits will be poorer because of the large dilution factor, very accurate analyses have been performed on mineral separates. Samples containing high concentrations of elements of normally low crustal abundance may require dilution because the wavelength, as determined by the fixed slit in the instrument, was selected for trace analysis. The limits of detection for the example dissolution are presented in table 1.

Table 2 presents data for the four reference materials andesite AGV-1, granodiorite GSP-1, granite G-2, 
Table 1. Detection limits in a granite for elements normally reported using an acid digestion'

[In micrograms per gram, unless otherwise indicated]

Element Lioft Element Liait Elenent Limit Elenent Limit
(percent)

\begin{tabular}{llrrrrrrr}
\hline Al & 0.05 & Ag & 2 & Ge & 20 & V & 2 \\
Fe & 0.05 & As & 10 & La & 2 & V & 2 \\
Mg & 0.05 & Au & 8 & Li & 2 & Vb & 1 \\
Ca & 0.05 & Ba & 1 & Mn & 10 & Zn & 4 \\
Ha & 0.1 & Be & 1 & Ho & 2 & Pr & 10 \\
K & 0.1 & Bi & 10 & Nb & 4 & Nd & 20 \\
Ti & 0.01 & Cd & 2 & Ni & 2 & Sa & 50 \\
P & 0.01 & Ce & 4 & Pb & 4 & Eu & 2 \\
& & Co & 1 & SC & 2 & Gd & 10 \\
& & Cr & 1 & Sn & 4 & Tb & 20 \\
& & Cu & 1 & Sr & 2 & Oy & 4 \\
& & Go & 4 & Th & 4 & Ho & 4 \\
\end{tabular}

\footnotetext{
1 $\mathrm{HF}_{\mathrm{HCl}} \mathrm{HNO} \mathrm{H}_{3}, \mathrm{HClO}_{4}$; $\mathrm{SI}$ and $\mathrm{B}$ are lost in this approach to sample dissolution.

2The polychronator in Reston does not have a fixed channel for gold. However, a 0.5- Ebert anochroaator, which is part of the inductively coupled plasma spectrometer systea, enables measurements on gold and other elements not routinely determined.
}

and basalt BHVO-1. The precision of the method for concentrations higher than 10 times the detection limit is better than \pm 10 -percent relative standard deviation. The precision of the analysis of the same solution is approximately \pm 0.5 -percent relative standard deviation. The loss in precision is due to sampling the ground material, weighing errors, partial dissolution of resistate minerals, and daily calibration of the instrument. The instrumental precision of the scanning instrument is a \pm 2 -percent relative standard deviation. This difference is due to the use of an internal standard in the multichannel instruments that can correct for gas-flow and nebulizer fluctuations by using a simultaneous measurement. The accuracy of the method is determined by the calibration of the instrument. Standards are used each day to calibrate the sensitivity of the instrument for each element. These standards consist of reference basalt BHVO-1 and syenite SY-3 and a series of synthetic solutions containing elements that are not present at quantifiable levels in BHVO-1 or SY-3. If these standards are prepared improperly, a systematic error will result. Spectral interferences are corrected mathematically. Approximately 300 interference corrections must be made on each sample to determine 44 elements. If the correction is made improperly, then an artificial correlation between two elements will appear. The correction factors are determined from single-element solutions, and the most significant interference factors must be determined daily. However, because of drift in the optics and gas-flow rates, these factors can change rapidly. To help stabilize these sources of drift, the instrument in Denver is profiled automatically between samples (Layman and Lichte, 1982). This autoprofiler minimizes most of the error caused by drift in the optics but cannot eliminate the error. Massflow controllers at the three centers are used to stabilize the gas-flow rates.

\section{Fusion}

The requirement of presentation of a solution to the instrument requires that the sample first be dissolved. The acid dissolution described above will not dissolve such minerals as zircon, tourmaline, cassiterite, rutile, and chromite. The dissolution of these materials can be accomplished by using appropriate fluxing reagents and by dissolving the resultant glasses. The acid dissolution 
Table 2. Analysis of reference standards by inductively coupled plasma-atomic emission spectrometry

\begin{tabular}{|c|c|c|c|c|c|c|c|c|}
\hline \multirow[b]{3}{*}{ Element } & \multicolumn{8}{|c|}{ Concentration } \\
\hline & \multicolumn{2}{|r|}{$A G Y-1$} & \multicolumn{2}{|r|}{ GSP-1 } & \multicolumn{2}{|r|}{ G-2 } & \multicolumn{2}{|r|}{ BHVO-1 } \\
\hline & \multicolumn{2}{|c|}{ ICP-AES Accepted* } & \multicolumn{2}{|c|}{ ICP-AES ACCepted } & \multicolumn{2}{|c|}{ ICP-AES Accepted* } & \multicolumn{2}{|c|}{$\underline{\text { ICP-AES Accepted }}$} \\
\hline & \multicolumn{8}{|c|}{ Percentage } \\
\hline Al & 9.0 & 9.1 & 8.0 & 8.1 & 8.0 & 8.1 & 7.8 & 7.4 \\
\hline Ca & 3.6 & 3.53 & 1.5 & 1.4 & 1.4 & 1.4 & 8.5 & 8.1 \\
\hline Fe & 4.6 & 4.74 & 2.9 & 3.0 & 1.8 & 1.8 & 8.7 & 8.3 \\
\hline K & 2.4 & 2.42 & 4.5 & 4.6 & 3.6 & 3.7 & 0.46 & 0.44 \\
\hline $\mathrm{Mg}$ & 0.92 & 0.92 & 0.59 & 0.58 & 0.46 & 0.46 & 4.4 & 4.3 \\
\hline Na & 3.2 & 3.20 & 2.1 & 2.1 & 3.0 & 3.0 & 1.9 & 1.71 \\
\hline $\mathbf{P}$ & 0.21 & 0.22 & 0.14 & 0.12 & 0.06 & 0.06 & 0.12 & 0.12 \\
\hline \multirow[t]{2}{*}{ TI } & 0.55 & 0.635 & 0.35 & 0.40 & 0.24 & 0.28 & 1.6 & 1.6 \\
\hline & \multicolumn{8}{|c|}{ Parts Per Million } \\
\hline Mn & 730 & 760 & 300 & 320 & 250 & 260 & 1.300 & 1,300 \\
\hline Ag & $<2$ & 0.094 & $<2$ & 0.083 & $<2$ & 0.04 & $<2$ & 0.056 \\
\hline As & $<10$ & 0.8 & $<10$ & 0.09 & $<10$ & 0.25 & $<10$ & -- \\
\hline AU & 8 & -- & 8 & -- & 8 & -- & $<8$ & -- \\
\hline Ba & 1.200 & 1,200 & 1,300 & 1,300 & 1.900 & 1,900 & 140 & 135 \\
\hline Be & 2 & 2 & 1 & 1 & 2 & 2.4 & $<1$ & -- \\
\hline$B 1$ & $<10$ & 0.05 & $<10$ & 0.037 & $<10$ & 0.3 & $<10$ & 0.014 \\
\hline Cd & $<2$ & 0.09 & $<2$ & 0.06 & $<2$ & 0.039 & $<2$ & -- \\
\hline Ce & 66 & 71 & 420 & 360 & 160 & 160 & 35 & 39 \\
\hline Co & 16 & 16 & 7 & 7.8 & 5 & 5 & 50 & 45 \\
\hline $\mathrm{Cr}$ & 11 & 10 & 13 & 12 & 9 & 8 & 300 & 300 \\
\hline Cu & 58 & 59 & 31 & 33 & 10 & 10 & 140 & 140 \\
\hline Eu & $<2$ & 1.6 & $<2$ & 2.4 & $<2$ & 1.4 & 3 & 2 \\
\hline $6 a$ & 21 & 21 & 21 & 23 & 23 & 23 & 22 & 21 \\
\hline Ho & $<4$ & 0.6 & $<4$ & -- & $<4$ & 0.4 & $<4$ & -- \\
\hline La & 40 & 36 & 190 & 195 & 93 & 92 & 17 & 17 \\
\hline LI & 11 & 12 & 28 & 30 & 31 & 35 & 5 & 5 \\
\hline Mo & $<2$ & 3 & $<2$ & 1.5 & $<2$ & 0.9 & $<2$ & 1 \\
\hline Nb & 9 & 16 & 20 & 23 & 9 & 13 & 15 & 19 \\
\hline Md & 34 & 37 & 220 & 190 & 55 & 58 & 26 & 24 \\
\hline Ni & 17 & 15 & 8 & 8 & 3 & 3.5 & 120 & 120 \\
\hline $\mathrm{Pb}$ & 34 & 33 & 50 & 54 & 30 & 30 & $<4$ & -- \\
\hline Sc & 10 & 12.5 & 5 & 6.6 & 2 & 3.5 & 32 & 31 \\
\hline Sn & $<20$ & 3.6 & $<20$ & 5 & $<20$ & 1.4 & $<20$ & -- \\
\hline$S r$ & 640 & 660 & 230 & 240 & 470 & 480 & 420 & 420 \\
\hline Ta & $<40$ & 1.4 & $<40$ & 1 & $<40$ & 0.8 & $<40$ & 1.1 \\
\hline Th & 7 & 6.4 & 110 & 105 & 24 & 25 & $<4$ & 1 \\
\hline $\mathbf{U}$ & $<100$ & 1.95 & $<100$ & 2.1 & $<100$ & 2.1 & $<100$ & 0.4 \\
\hline v & 110 & 125 & 47 & 54 & 30 & 36 & 310 & 320 \\
\hline $\mathbf{v}$ & 18 & 19 & 24 & 29 & 8 & 11 & 26 & 27 \\
\hline$r_{b}$ & 2 & 1.9 & 1 & 1.9 & $<1$ & 0.86 & 2 & 1.9 \\
\hline $2 n$ & 87 & 86 & 100 & 105 & 86 & 84 & 100 & 105 \\
\hline
\end{tabular}

abbey (1983). 
method also is not appropriate for the determination of silicon and boron because of their volatility as fluorides, but these elements can be determined by using fusions for the dissolution. Commonly used reagents include $\mathrm{Na}_{2} \mathrm{CO}_{3}$, $\mathrm{LiBO}_{2}, \mathrm{Na}_{2} \mathrm{O}_{2}$, persulfates, $\mathrm{NaOH}$, and $\mathrm{KOH}$. The choice often is dictated by the acidic or basic nature of the rocks and the elements of interest. $\mathrm{LiBO}_{2}$ is appropriate for silicon analysis but precludes the analysis of boron or lithium.

An example of the fusion dissolution is the determination of major element analysis in silicate rocks and minerals (Brookes and others, 1981). The instrumental parameters for this method were optimized by using a sequential simplex method (Leary, Brookes, Dorrzapf, and Golightly, 1982). This analysis is used primarily in Reston and offers an alternative to conventional rapid rock procedures. In this method, 10 elements, $\mathrm{Al}, \mathrm{Ca}, \mathrm{Fe}$, $\mathbb{K}, \mathrm{Mg}, \mathrm{M} n, \mathrm{Na}, \mathbb{P}, \mathrm{Si}$, and $\mathrm{Ti}$, are determined simultaneously.

In this method, $100 \mathrm{mg}$ of -100 mesh silicate rock is mixed with $0.6 \mathrm{~g}$ of $1: 2$ lithium metaborate-lithium tetraborate and fused at $1,000^{\circ} \mathrm{C}$ for $45 \mathrm{~min}$. Lithium tetraborate is a more acid flux than the metaborate and, thus, produces fusion beads that do not adhere to the walls of graphite crucibles when the ferric oxide concentration exceeds 15 percent. The resulting fusion bead is removed from the graphite crucible and dissolved in dilute nitric acid. An aliquot of indium solution is added, and the final volume of solution is adjusted to $250 \mathrm{~mL}$. The indium, 20 $\mu \mathrm{g} / \mathrm{mL}$ in the final solution, serves as an internal standard that corrects for possible nebulizer drift. The nebulization process is controlled through use of a mass-flow controller for regulating the flow rate of argon into the nebulizer and injection tube of the plasma torch and through the use of a peristaltic pump that delivers the sample at a constant $0.81 \mathrm{~mL} / \mathrm{min}$. The low concentration of total dissolved solids, $2.8 \mathrm{mg} / \mathrm{mL}$, minimizes the risk of clogging the fixed cross-flow pneumatic nebulizer. Currently, a set of four matural standards (granite $G-2$, basalt $\mathbb{B H V O}-1$, andesite $A G V-1$, and basalt $\mathbb{B I R}-1$ ) is being used for calibration of the ICP spectrometer system. From the moment a sample solution is introduced into the ICP to the end of the measurement cycle for the 10 elements requires approximately $2 \mathrm{~min}$. This capability of making rapid, simultaneous measurements enables the automated analysis of large batches of sample solutions during an 8-h interval. Within such a long series of measurements, the quality of measurements is ascertained by placing solutions of standard rocks at locations between groupings of 10 sample solutions. Results of measurements on these control standards serve as a realtime indicator of the stability of the $\mathbb{I C P}$ spectrometer system and provide a basis for judging the quality of each elemental determination.
Typically, the routine precision of analysis is a 3- to 5 -percent relative standard deviation. The routine accuracy of analysis can be the same as the precision, but, in some cases, the presence in samples of mineral phases resistive to fusion and dissolution will cause significant systematic errors. In these instances, fusions with another salt, such as sodium peroxide or sodium hydroxide, may be necessary.

This procedure can be modified for small sample sizes or for other groups of elements. The large dilution of the sample is appropriate for major elements but does raise the limits of detection by a factor of 50 . When using fusion procedures for dissolution, a practical dilution of 200:1 can be used. The limits of detection are four to five times higher than those listed in table 1 using the acid digestion, but they can be significantly improved through chemical preconcentration procedures.

\section{QWองิ@ Anglysis}

For samples that are already liquid, sample preparation becomes much easier. These samples can be analyzed directly, after dilution or preconcentration. The total dissolved solids in the sample should not be greater than about 2 percent. Thus, most river and spring waters can be concentrated by evaporation, and brines must be diluted. A typical procedure for waters is to evaporate 50 $\mathrm{mL}$ of water to near dryness and to dilute it to $2.5 \mathrm{~mL}$ by using 10 percent aqua regia.

A method developed in $\mathbb{R}$ eston for the determination of $\mathrm{Ca}^{2+}, \mathrm{Mg}^{2+}, \mathrm{Na}^{+}$, and $\mathrm{K}^{+}$in fluid inclusions uses sample volumes from 0.2 to $40 \mu \mathrm{L}$. The sample is diluted to $1 \mathrm{~mL}$ and split for analysis by ion chromatography for $\mathrm{Br}-, \mathrm{Cl}^{-}, \mathrm{F}^{-}$, and $\mathrm{SO}_{4}{ }^{2-}$; the other split is diluted $1: 1$ with 10 percent $\mathrm{HCl}$ for analysis by $\mathrm{ICP}$ spectrometry. Anionto-cation balances of $1.06 \pm 0.10$ have been obtained for selected groups of inclusions (Roedder and others, 1987).

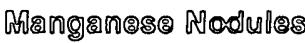

An example of the need for versatility in wavelength selection is demonstrated in the analysis of manganese modules for trace elemental content (Fries and others, 1984). For this analysis, more than 70 wavelengths have been evaluated with the goal of minimizing spectral interferences primarily from manganese in the modules. An acid digestion is employed to dissolve the nodule, and ICP-AES spectrometric analysis uses the wavelengths chosen based on optimum sensitivity and minimal interference. The data presented in table 3 show the results for U.S. Geological Survey manganese nodule standards A-1 and $\mathbb{P}-1$. The results are in good agreement with those obtained by spark-source mass spectrometry and neutron activation analysis. The precision for most elements is a 
Table 3. Concentrations determined by neutron activation analysis (NAA), spark-source mass spectrometry (SSMS), and inductively coupled plasmaatomic emission spectrometry (ICP-AES) of U.S. Geological Survey manganese nodule standards $\mathrm{A}-1$ and $\mathrm{P}-1$

Concentration (ug/g)

\begin{tabular}{|c|c|c|c|c|c|c|c|c|}
\hline \multirow[t]{2}{*}{ Elewent } & \multicolumn{4}{|c|}{$A-1$} & \multicolumn{4}{|c|}{$P-1$} \\
\hline & $\operatorname{MAA}^{2}$ & S.S.M. $s^{2}$ & ICP-AES & $\begin{array}{c}\text { PSD } \\
\text { percent }\end{array}$ & MAA $^{2}$ & S.S.M. $S^{2}$ & ICP-AES & $\begin{array}{c}\text { RSO } \\
\text { percent }\end{array}$ \\
\hline
\end{tabular}

$\begin{array}{lcccccccc}\text { La } & 132.5 & 130 & 104 & 1.9 & 120 & 82 & 100 & 2.0 \\ \text { Ce } & 668 & 2300 & 760 & 1.7 & 289 & 280 & 310 & 3.5 \\ \text { Pr } & -- & 23 & <30 & -- & -- & 27 & <30 & -- \\ \text { Hd } & 85.3 & 94 & 81 & 2.5 & 112.8 & 110 & 124 & 1.6 \\ \text { Su } & 20.9 & 21 & 18 & 11 & 30.4 & 28 & 20 & 15 \\ \text { Eu } & 4.48 & 4.8 & 4.3 & 2.3 & 6.57 & 6.8 & 7.0 & 2.9 \\ \text { Ed } & 26.5 & 22 & 29 & 6.9 & 29.4 & 24 & 21 & 19 \\ \text { Tb } & 4.87 & 3.8 & <10 & -- & 5.31 & 4.2 & <10 & -- \\ \text { Dy } & -- & 22 & 20 & 25 & -- & 25 & 28 & 18 \\ \text { Ho } & -- & 5.3 & 5.8 & 12 & -- & 5.1 & 6.5 & 12 \\ \text { Er } & -- & 15 & 14.6 & 6.2 & -- & 13 & 13.6 & 4.4 \\ \text { T. } & 1.72 & -- & <5 & -- & 1.77 & -- & <5 & -- \\ \text { Yb } & 16.3 & 13.5 & 13.2 & 1.5 & 13.8 & 13 & 11.8 & 2.5 \\ \text { Lu } & 2.16 & -- & 2.8 & 14 & 1.85 & -- & 1.7 & 18 \\ \text { Y } & 115 & -- & 97 & 2.1 & 89 & -- & 75 & 2.7 \\ \text { Sc } & 11.23 & -- & 10.8 & 1.9 & 9.47 & -- & 9.3 & 3.2 \\ & & & & & & & & \end{array}$

\footnotetext{
IFlanagan and cottfled (1980).

${ }^{2}$ Rankin and others (1979, p. 684-685).
}

2- to 5-percent relative standard deviation. As the concentration of an element approaches the limit of detection, the relative standard deviation is higher, such as indicated for samarium, gadolinium, and dysprosium.

\section{Chemical Separation}

An alternative method to decrease the occurrence of spectral interferences and to increase sensitivity is to separate chemically the trace elements from the matrix. This can be accomplished through liquid-liquid extraction, such as an ADPC-MIBK extraction for lead and cad- mium, or through ion exchange procedures. The analysis of the rare-earth elements is one example (Crock and Lichte, 1982). In this method, $1 \mathrm{~g}$ of sample is dissolved by using an acid digestion and diluted in $1 \mathrm{~N} \mathrm{HNO}_{3}$. This solution is loaded onto a $20-\times 1-\mathrm{cm}$ column of $100-200$ mesh AG50W-X8 cation resin and eluted with $2 \mathrm{~N} \mathrm{HNO}_{3}$ acid followed by $6 N$ and $8 N \mathrm{HNO}_{3}$ elutions. The $6 N$ and $8 N$ elutions contain the rare-earth elements and iron. The iron is removed by an anion column (AG1-X8; $5 \times 1 \mathrm{~cm}$ ) from an $8 \mathrm{~N} \mathrm{HCl}$ solution. The solution containing only rare-earth elements is evaporated to dryness and dissolved with 5 or $10 \mathrm{~mL}$ of 30 percent $\mathrm{HCl}$. Cadmium is added at $10 \mathrm{mg} / \mathrm{L}$ to serve as an internal standard. The results and limits of detection are presented in table 4. 
Table 4. Rare-earth analysis by inductively coupled plasma-atomic emission spectrometry (ICP-AES) of U.S. Geological Survey standard basalt BCR-1

[In parts per million]

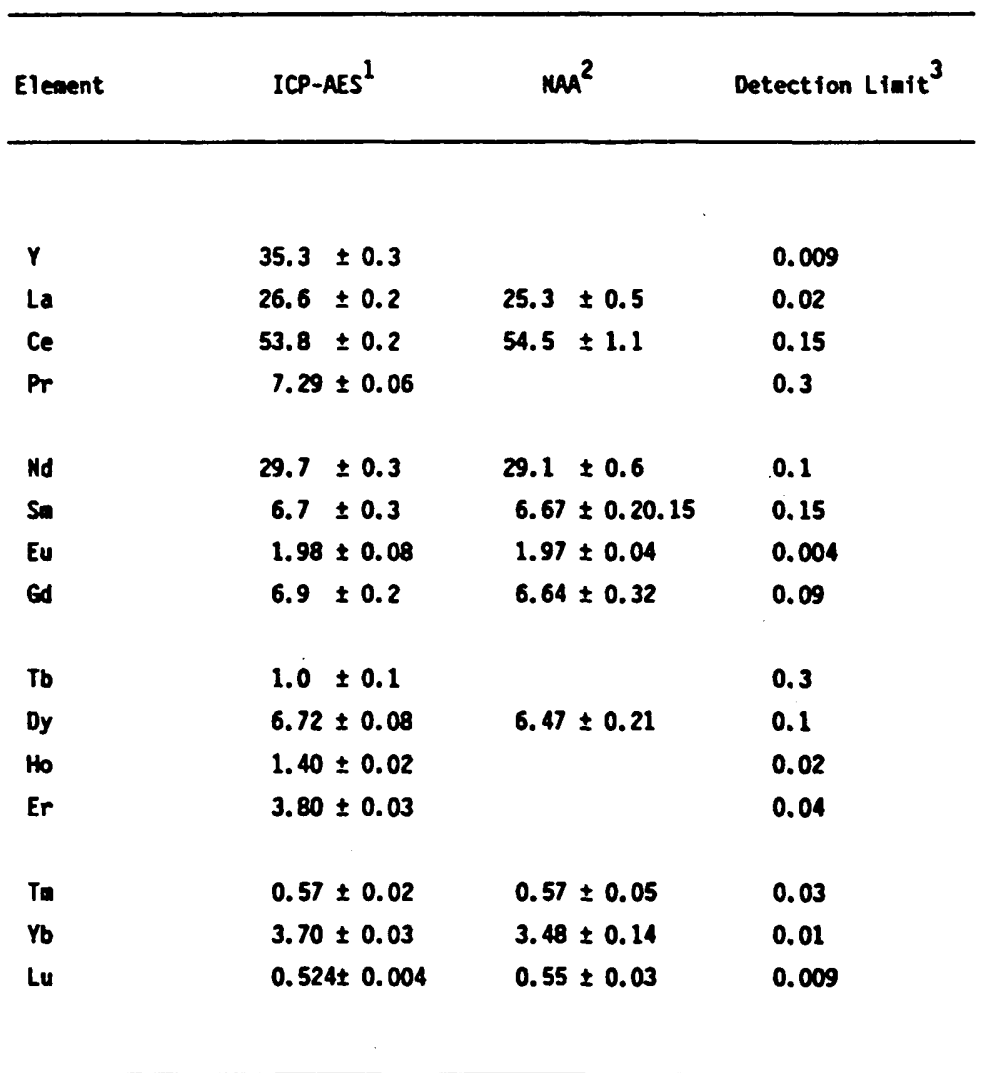

\footnotetext{
1 Crock and Lichte (1982).

2Hugh Millard (1985). U.S. Geological Survey, Denver, Colorado, written comunication. (NAA - Neutron activation analys is).

${ }^{3}$ Inductively coupled plasma-atomic emission spectronetry for $1-g$ sample diluted to a 5-al final voluae.
}

\section{Selective Extraction}

One advantage of analyzing solutions is the ability to dissolve specific minerals selectively. An example of selective extraction is the $\mathrm{HCl}: \mathrm{H}_{2} \mathrm{O}_{2}$ dissolution of sulfide minerals and the determination of $\mathrm{As}, \mathrm{Bi}, \mathrm{Cd}, \mathrm{Sb}$, and $\mathrm{Zn}$. This method uses $0.15 \mathrm{~g}$ of -100 -mesh material. Volumes of $3.0 \mathrm{~mL}$ of concentrated $\mathrm{HCl}$ and $0.2 \mathrm{~mL}$ of 30 percent $\mathrm{H}_{2} \mathrm{O}_{2}$ are added and mixed on a vortex shaker. The sample is heated to $100^{\circ} \mathrm{C}$ for $30 \mathrm{~min}$ and allowed to cool and settle overnight. Lutetium is used as an internal standard, and the solution is analyzed directly from the extraction test tube. A Babington-type nebulizer is used for the analysis because of the particles suspended in the solution. Even though only elements are reported, the concentrations of 50 elements in solutions must be determined to apply the correction factors for the spectral interferences. The precision of this method is an approx- imately 10-percent relative standard deviation. Accuracy is not well defined for this type of procedure because only certain minerals will be dissolved.

\section{CAPABILITIES AND UMITATIONS}

The examples discussed in the "Experimental" section give some of the capabilities of this technique. Although the ICP emission source has the capability of exciting virtually every element, only about 50 elements are compatible with the total method; for instance, the sensitivity of the technique varies with every element, and not all elements are compatible in a single solution.

For many elements, the detectability is adequate to measure concentrations at the crustal abundance level. 
For those cases where preconcentration is necessary to detect background levels, the liquid sample introduction mechanism is ideally suited. Elements not generally analyzed are $\mathbb{H}, C, N, O, \mathbb{F}, C l, B r, S$, and II because of their presence in air and dissolution reagents. Measurement capabilities for $\mathbb{R b}$ and $C s$ are limited because of poor sensitivity. The rest of the naturally occurring elements can be analyzed if they are present in minerals that can be dissolved.

The dissolution procedure is by far the most timeconsuming step of the analysis. Some samples may require more than one dissolution procedure to dissolve and determine all the elements of interest. The plasma takes on different characteristics with high salt-content solutions. Therefore, samples requiring a fusion technique for dissolution will yield poorer limits of detection because of the larger dilution than required for the acid digestion to achieve similar total salt content.

A fundamental limitation of the ICP-AES technique arises from its greatest asset. The excitation energy of the plasma is high enough to excite ground state and ion electron transitions for most elements. Thus, for typical instruments employing optics of moderate dispersion, the opportunity for spectral overlap from other elements in the sample is much greater than from less energetic sources, such as the $d-c$ arc or an air-acetylene flame. The spectral overlap contribution from each element is corrected mathematically. However, the degree of overlap and the concentration of the interfering element combine to deteriorate the limit of detection. Thus, the limit of detection depends not only on the dilution factor of the sample caused by dissolution but also on the concentrations of other elements in the sample that offer spectral overlap.

The ICP-AES technique is highly precise. The instrumental precision of measurement for concentrations well above detection limits is 1- to 2-percent relative standard deviation. In most cases, sampling and dissolution steps limit the precision. Sampling depends on how thoroughly the sample is ground and mixed, and the dissolution precision is dependent on the presence of resistant minerals that may be attacked only partially by the reagents. The precision of most determinations will be a \pm 5 - to 10-percent relative standard deviation with the optimal limit of 1 to 2 percent.

Any method that is highly precise also can be highly accurate if the samples are compared to the proper standards. The ICP-AES method is a secondary method (that is, reference standards are necessary for determining its sensitivity), but the high temperatures of the plasma greatly reduce the effects of matrix elements on the slope of the calibration curves, and, for most samples, the accuracy of the amalysis is nearly equal to the precision. However, standardization of the method relies on standards prepared separately and then applied to the sample analysis. Reference standards remain important for verification of the accuracy of the technique. Because dissolution of the minerals is important and the ICP-AES technique only analyzes the final solution, the reference material must match the mineral content and the major and minor elemental concentrations.

\section{REFERENCES CITED}

Abbey, Sydney, 1983, Studies in "Standard Samples" of silicate rocks and minerals, 1969-1982: Geological Survey of Canada, Paper 83-15, 114 p.

Brookes, A. E., Leary, J. J., and Golightly, D. W., 1981, Selection of operating conditions for multiple-element analysis by inductively coupled plasma spectrometry-An application of optimization methodology in analytical chemistry: U.S. Geological Survey Open-File Report 81-1211, $80 \mathrm{p}$.

Crock, J. G., and Lichte, F. E., 1982, The determination of rare-earth elements in geological materials by inductively coupled argon plasma-optical emission spectroscopy: Analytical Chemistry, v. 54, p. 1329-1333.

Crock, J. G., Lichte, F. E., and Briggs, P. H., 1983, Determination of elements in National Bureau of Standards geological reference materials SRM 278 obsidian and SRM 688 basalt by inductively coupled argon plasma-atomic emission spectroscopy: Geostandards Newsletter, v. 7, no. 2, p. 335-340.

Flanagan, F. J., and Gottfried, D., 1980, USGS Rock Standards III: Manganese nodule reference samples USGS Nod-A-1 and USGS Nod-P-1: U.S. Geological Survey Professional Paper 1155, $39 \mathrm{p}$.

Fries, T. L., Lamothe, P. J., and Pesek, J. J., 1984, Determination of rare-earth elements in manganese nodules by inductively coupled argon-plasma emission spectroscopy: Analytica Chimica Acta, v. 159, p. 329-336.

Golightly, D. W., 1978, Recent geochemical applications of the inductively coupled plasma-Applications of inductively coupled plasmas to emission spectroscopy, 1977, New York, Eastern Analytical Symposium, R. M. Barnes, ed.: The Franklin Institute Press, Philadelphia, p. 107-119.

Kane, J. S., 1985, An inductively coupled plasma spectrometric method for the determination of six indicator elements for mineralization processes in granites: Geostandards Newsletter, v. 9, no. 2 , p. 181-190.

Layman, L. R., and Lichte, F. E., 1982, Design and implementation of an auto-profiling system for spectrometers using ICP excitation: ICP Newsletter v. 7, no. 11, p. 565.

Leary, J. J., Brookes, A. E., Dorrzapf, A. F., Jr., and Golightly, D. W., 1982, An objective function for optimization techniques in simultaneous multiple-element analysis by inductively coupled plasma spectrometry: Applied Spectroscopy, v. 36, p. $37-40$.

Rankin, P. C., and Glasby, G.P., 1979, Regional distribution of rare-earth and minor elements in manganese nodules and associated sediments in the southwest Pacific and other localities, in Bischoff, J. L., and Piper, D. Z., eds., Marine 
Geology and Oceanography at the Pacific Manganese Nodule Province: Plenum Press, New York, p. 684-685.

Roedder, Edwin, d'Angelo, W.M., Dorrzapf, A.F., Jr., and Aruscavage, P.J., 1987, Composition of fluid inclusions in permian salt beds, Palo Duro basin, Texas, U.S.A.: Chemical Geology, v. 61, p. 79-90.
Taggart, J. E., Lichte, F. E., and Wahlberg, J. S., 1981, Methods of analysis of samples using $\mathrm{X}$-ray fluorescence and induction coupled plasma spectroscopy in the 1980 eruptions of Mount St. Helens, Washington: U.S. Geological Survey Professional Paper 1250, p. 683-687. 


\section{CHAPTER C}

Atomic Absorption Methods

By P. J. ARUSCAVAGE and J. G. CROCK

U.S. GEOLOGICAL SURVEY BULLETIN 1770

Methods for Geochemical Analysis 


$$
\text { . }
$$




\section{CONTENTS}

Abstract $\mathbf{C 1}$

Introduction $\mathbf{C 1}$

Fundamental principles of atomic absorption spectroscopy C1

Experimental practice C2

High-sensitivity atomic absorption methods C3

Geologic considerations and limitations for atomic absorption spectroscopy C3

Summary C6

References cited C6

\section{TABLE}

1. Approximate determination limits in silicate rocks by various techniques C4 


\title{
Atomic Absorption Methods
}

\author{
By P. J. Aruscavage and J. G. Crock
}

\begin{abstract}
The fundamental principles of atomic spectrometry are outlined and followed by a brief description of normal experimental practices used in the technique. A general description of some of the common interferences encountered in routine determinations and some of the methods used to correct or minimize them are given. The high-sensitivity techniques of graphite furnace atomic absorption spectrometry and hydride-genieration atomic absorption spectrometry are described briefly, as well as some of the limitations of the atomic absorption methods with respect to geological applications. Finally, a table is presented comparing the various atomic absorption methods with the newer inductively coupled plasma-atomic emission spectrometry technique that shows which of the methods might be appropriate for determining average abundances of lithophilic elements.
\end{abstract}

\section{INTRODUCTION}

The first atomic absorption spectrometer was purchased by the Analytical Laboratories of the Geobgic Division about a decade after Walsh's (1955) classic publication, in which he proposed flame atomic absorption spectrometry (FAAS) as a useful technique for the determination of metallic elements. Since that time, atomic absorption spectrometry (AAS) has become one of the most widely used techniques for determining major and trace metallic elements in geologic materials. Among the reasons for its popularity are

1. Relatively low purchase and maintenance costs,

2. Simplicity of operation and maintenance,

3. Capability for determining about 70 elements,

4. High sensitivity for many elements,

5. Speed of determination,

6. Relative freedom from interference,

7. Good precision and accuracy, and

8. Portability.

However, the method has the following limitations:

(1) the technique is not useful for determining nonmetals,

(2) the refractory elements are determined with poor sensitivity, (3) simultaneous multielement analysis has not been practical, and (4) the precision is generally poorer than volumetric or gravimetric methods.
Except for some improvements in lamp design, electronics, and data handling, the basic design of atomic absorption instrumentation has not changed over the last two decades. However, these improvements, along with the introduction of background correction capability and the high-sensitivity methods (hydride atomic absorption and graphite furnace atomic absorption), have extended the applicability of AAS and, at the same time, made the technique faster, more precise, and more accurate.

In the Geologic Division laboratories, 35 metallic elements are determined routinely by AAS. The technique now replaces the more laborious colorimetric, volumetric, and gravimetric methods in some instances and extends the capabilities for determining other elements.

\section{FUNDAMENTAL PRINCIPLES OF ATOMIC ABSORPTION SPECTROMETRY}

The fundamental principles underlying AAS are well described in many books on atomic spectroscopy (Robinson, 1966; Slavin, 1968; Schrenk, 1975; and Welz, 1976). In summary, every atomic absorption spectrometer has a light source, light-modulation system (chopper), sample atomization system, and a light-selectionmeasurement system.

The light source, generally a hollow cathode or electrodeless discharge lamp, functions as the energy source that emits light of certain wavelengths, which can be absorbed uniquely by the element of interest. The chopper separates the emission of light from the excited atoms within the sample cell from the light source itself, thus allowing the difference in intensity measurements (absorbance) to be made accurately. The sample atomization cell, generally a flame, electrically heated graphite furnace, or heated quartz cell, uses thermal energy to dissociate the molecules into gaseous atoms that are capable of absorbing specific light energy from the light source. The light-selection-measurement system, which includes the slits, monochrometer, photomultiplier-amplifier system, and readout system, selects the proper wavelength, measures the light intensity variation, amplifies the electrical signal, and records the data. 
Quantitative determination by AAS is made possible or feasible because of the following fundamental reasons:

1. Every element has a unique electronic structure.

2. The wavelength of light (energy) emitted or absorbed by an atom is a unique property of each element.

3. High-intensity monoatomic light sources have been developed.

4. The amount of light quanta absorbed is proportional to the amount of free atoms in the light path.

5. Instrumentation has been developed that can select and isolate the appropriate wavelength, generate ground state (or resonance state) free atoms, and detect, amplify, and record the change in light intensity (absorption) due to these free atoms.

\section{EXPERIMENTAL PRACTICE}

From the Beer-Lambert equations describing absorption phenomena,

$$
\text { Absorption }=\log \frac{I_{o}}{I}
$$

and

$$
\text { Absorbance }=a b c,
$$

where

$$
\begin{aligned}
& I_{o}=\text { intensity before absorption, } \\
& I=\text { intensity after absorption, } \\
& a=\text { absorption coefficient }=\text { constant for each ele- } \\
& \quad \text { ment, } \\
& b=\text { length of absorption cell }=\text { constant, and } \\
& c=\text { concentration of free atoms in cell. }
\end{aligned}
$$

Because of the linear relation of absorbance with concentration in equation 2 , absorbance has become the most useful of the two equations to characterize light absorption in AAS. Equation 2 describes absorbance in absolute terms, whereas, in practice, AAS is a comparative method where the absorbance of known concentrations are measured and the concentrations of the unknowns are determined by comparison according to the equation,

$$
\begin{gathered}
\text { concentration (unknown) }=\text { concentration (known) } \\
\frac{\text { absorbance (unknown) }}{\text { absorbance (known). }}
\end{gathered}
$$

In most modern AAS instrumentation, the calibration curves that are generated are stored in a computer within the instrument and a direct readout of the unknown concentration can be made.

Although AAS is almost free of spectral interferences because of the specificity of light absorption, interferences exist that must be recognized and controlled before accurate determinations can be made. These interferences generally fall into one of four categories-matrix, chemical, ionization, and nonspecific light absorption (background absorption).
An example of a matrix or physical interference is the change in nebulization efficiency caused by differences in the viscosity or surface tension between calibration standards and real samples. This type of interference is usually minimized by matrix matching the calibration standards to the samples or by dilutions to the point where the interference is negligible.

Chemical interference occurs when another molecule or element within the analyte solution combines or reacts with the analyte in such a way that the atomization efficiency varies between the calibration standards and the samples. An example is the reaction of phosphate ions with calcium ions to produce the compound calcium phosphate, which is not dissociated efficiently in the air-acetylene flame. This type of interference generally is removed by using the hotter nitrous oxide-acetylene flame or by adding a releasing agent, such as lanthanum, which reacts with the phosphate ions and, thereby, releases the calcium ions for efficient atomization.

Ionization interferences can occur when the number of free electrons within the sample cell is different than those of the calibration standards and the samples. This type of interference, which occurs in alkali and alkaline earth determinations, is caused by an excess amount of energy from the sample cell (flame) that excites the ground state (or resonance state) to an ionized state. By adding a large excess of an easily ionizable element to standards and samples to create a constant amount of free electrons in the flame, ionization interference can be controlled. Thus, in the determination of $\mathrm{Na}, \mathrm{Li}$, or $\mathrm{Ba}, \mathrm{a}$ large excess of $K$ is added to the standards and the samples.

Nonspecific light absorption occurs when molecules, which absorb light over a broad energy range, absorb some of the light from the light source or when particles within the sample cell scatter some of the light passing through the cell. This type of interference is very prevalent in graphite furnace atomic absorption spectroscopy (GFAAS). Most modern instrumentation now has the capability for automatically correcting for this type of broadband interference by using continuum, Zeeman, or Smith-Heiftje background correction systems, each of which has its own advantages and disadvantages.

An often-used alternative to eliminate matrix, chemical, and ionization interferences is to correct for them by using the method of standard additions. In this method, several aliquots of the liquid sample are taken, and known amounts (spikes) of the analyte are added to each aliquot. After plotting the absorbance data against concentration of the known spike added and by extrapolating the linear portion of the calibration line backward from zero on the concentration axis, the concentration of the unknown can be determined. The presence of an interference is confirmed if the slope of the spiked sample curve is different from that of the pure standard curve. 
The method of standard additions will not correct accurately for interference if

1. The sample analyte and added spike are not equilibrated with the interfering species,

2. Nonspecific absorbance occurs, and

3. The linear portion of the calibration curve is exceeded.

Because of the inefficiency of analyzing large numbers of samples by this method on a routine basis, all other methods of interference elimination, including chemical separation of the analyte from the matrix, generally are preferred.

\section{HIGH-SENSITIVITY ATOMIC ABSORPTION METHODS}

Generally FAAS is limited to determinations in the part-per-million and above range in rock samples (see table 1). To extend the determination limits to lower levels, chemical separation and concentration procedures and (or) one of the high-sensitivity AAS techniques must be used. The two most widely used high-sensitivity AAS techniques are the hydride-generation AAS (HGAAS) and the GFAAS. Mercury is determined with high sensitivity using a cold-vapor AAS technique.

The HGAAS method is limited to those elements that form volatile hydrides; namely, $\mathrm{As}, \mathrm{Sb}, \mathrm{Se}, \mathrm{Te}, \mathrm{Bi}$, and $\mathrm{Sn}$. The sample generally is treated with sodium borohydride, and the evolved hydride is passed through the sample cell (flame or heated quartz tube) in a stream of nitrogen or argon gas. Typical detection limits are in the low part-per-billion range in rocks; however, because of the possibility of interference from many heavy and transition metals, great care must be followed when using this technique for geologic samples (Briggs and Crock, 1986; Crock, 1986; Crock and Lichte, 1982; Pierce and Brown, 1976, 1977).

Except for the highly refractory elements, the GFAAS technique can determine all the elements determinable by FAAS in the part-per-billion range in rocks. In the general method, a small liquid aliquot (typically $0.02-0.05 \mathrm{~mL}$ ) is added to the graphite tube, which is placed in the optical path. The sample then is dried at $100-120^{\circ} \mathrm{C}$, charred at an intermediate temperature $\left(500-1,000^{\circ} \mathrm{C}\right)$, and, finally, atomized in an argon atmosphere at a high temperature $\left(2,000-2,700^{\circ} \mathrm{C}\right)$ by passing a large electric current through the graphite tube. The peak-shaped absorbance curve then is measured, and the concentrations of the unknown are determined by the comparative method.

However, as with the HGAAS method, great caution must be exercised when analyzing geologic samples by this method because of the greater amount of chemical, matrix, and background interferences prevalent in GFAAS. In fact, chemical separation procedures are used extensively before determining many elements by this technique because of the difficulty of completely eliminating all interferences from the rock matrix (Aruscavage and Campbell, 1979a, b, 1977; Simon and others, 1977).

The determination of mercury is made uniquely by the highly selective and sensitive cold-vapor atomic absorption technique. The mercury in solution is reduced to the metal with stannous chloride in an aeration flask, carried in a stream of nitrogen gas, and amalgamated with gold chips contained in a quartz tube, which is located axially within the coil of an induction furnace. Rapid heating of the gold releases the mercury which is carried into a quartz cell where its atomic absorption signal is measured. A more detailed discussion of the hydride-generation and the mercury methods can be found in Chapter D.

\section{GEOLOGIC CONSIDERATIONS AND LIMITATIONS FOR ATOMIC ABSORPTION SPECTROMETRY}

In its various forms, AAS has proved to be a very versatile and useful technique for determining elements in geologic materials. However, the analytical chemist must have a thorough knowledge of possible interferences due to the rock matrix and a knowledge of methods for their correction or elimination before accurate determinations are possible. In addition, a knowledge of and skill in various sample decomposition methods for geologic materials and the ability to choose the most appropriate method for the element(s) to be determined are essential.

For most elements, it is usually desirable to decompose samples with acids such as $\mathrm{HF}, \mathrm{HNO}_{3}, \mathrm{HCl}, \mathrm{HClO}_{4}$, and $\mathrm{H}_{2} \mathrm{SO}_{4}$. The reasons for this are threefold. First, sample decomposition by acids is generally simpler and more rapid than fusions. Second, the blanks are usually lower. Third and foremost, lower determination limits generally are attained because of the reduced amount of dissolved solids.

However, when acid attack is insufficient to decompose the sample or when volatility losses occur with acid digestion, a fusion with a suitable flux is necessary. When this is required, poorer determination limits usually are encountered because of the larger dilution factors required to keep the dissolved salt content in the 1- to 2-percent range.

Knowledge by the analyst of the type of material to be analyzed is important not only for choosing a proper decomposition method, but for choosing the proper AAS technique and control standards.

Table 1 shows the average lithosphere abundance of elements and determination limits by various techniques. It should be kept in mind that the determination limits are calculated and that they are generally higher in the routine analysis of complex high salt rock solutions. From the table, 
Table 1. Approximate determination limits in silicate rocks by various techniques

[In micrograms per gram]

\begin{tabular}{|c|c|c|c|c|c|}
\hline Eleaent & $\begin{array}{c}\text { Average } 1 \\
\text { lithosphere } \\
\text { abundance }\end{array}$ & FAAS $^{2}$ & GFAAS $^{3}$ & HCANS $^{4}$ & ICP-AES 5 \\
\hline Ag & 0.02 & 2 & 0.015 & & 1 \\
\hline Al & 81,300 & 40 & 0.1 & & 10 \\
\hline As & 5 & 32 & 0.1 & 0.1 & 15 \\
\hline Au & 0.001 & 10 & 0.05 & - & 3 \\
\hline B & 10 & 600 & 5 & & 2 \\
\hline Ba & 430 & 16 & 0.1 & & 0.2 \\
\hline Be & 6 & 1 & 0.005 & & 0.3 \\
\hline Bi & 0.2 & 20 & 0.05 & 0.03 & 30 \\
\hline Ca & 36,300 & 3.2 & 0.01 & & 0.1 \\
\hline Cd & 0.18 & 1.0 & 0.003 & & 1 \\
\hline co & 40 & 6 & 0.10 & & 2 \\
\hline cr & 200 & 4 & 0.03 & & 2 \\
\hline cs & 3.2 & 4 & --- & ' & -- \\
\hline Cu & 70 & 3.6 & 0.05 & & 2 \\
\hline Dy & 4.47 & 34 & -.- & & 2 \\
\hline Er & 2.47 & 38 & --- & & 5 \\
\hline Eu & 1.06 & 22 & -.- & & 1 \\
\hline Fe & 50,000 & 4.8 & 0.05 & & 1 \\
\hline Ga & 15 & 52 & 0.3 & & 20 \\
\hline Gd & 6.36 & 640 & $\ldots$ & & 8 \\
\hline 6 & 7 & 96 & 0.1 & & so \\
\hline Hf & 4.5 & 600 & -- & & 5 \\
\hline $\mathrm{Hg}$ & 0.5 & 300 & 1 & 0.02 (cold vapor) & 50 \\
\hline Ho & 1.15 & 44 & --- & & 5 \\
\hline In & 0.1 & 30 & 0.25 & & 30 \\
\hline Ir & 0.001 & 320 & 1.5 & & so \\
\hline$k$ & 25,900 & 1.8 & 0.005 & & 80 \\
\hline La & 18.3 & 1,800 & --- & & 6 \\
\hline LI & 65 & 1.4 & 0.015 & & 1 \\
\hline Lu & 0.75 & 240 & $\cdots$ & & 5 \\
\hline Mg & 20,900 & 0.28 & 0.0015 & & 0.1 \\
\hline Mn & 1,000 & 2.2 & 0.015 & & 0.5 \\
\hline Mo & 2.3 & 20 & 0.05 & & 5 \\
\hline Ma & 28,300 & 0.6 & 0.005 & & 4 \\
\hline Nb & 20 & 1,480 & $\cdots$ & & 20 \\
\hline Nd & 23.9 & 400 & -- & & 10 \\
\hline Mi & 100 & 6 & 0.05 & & 4 \\
\hline os & --- & 40 & -- & & 100 \\
\hline $\mathbf{p}$ & 1,200 & 10,000 & 0.25 & & 30 \\
\hline $\mathrm{Pb}$ & 16 & 20 & 0.05 & & 15 \\
\hline Pd & 0.01 & 10 & 0.8 & & 40 \\
\hline $\mathrm{Pr}$ & 5.53 & 2.200 & -.- & & 15 \\
\hline Pt & 0.005 & 40 & 0.5 & & 20 \\
\hline
\end{tabular}


Table 1. Approximate determination limits in silicate rocks by various techniques-Continued

\begin{tabular}{|c|c|c|c|c|c|}
\hline Element & $\begin{array}{c}\text { Average } \\
\text { lithosphere } \\
\text { abundance }\end{array}$ & FAAS $^{2}$ & GFAAS ${ }^{3}$ & HEAAS 4 & ICP-AES $^{5}$ \\
\hline Rb & 280 & 4 & 0.1 & & $\cdots$ \\
\hline Re & 0.001 & 600 & --- & & 20 \\
\hline Rh & 0.001 & 12 & 0.8 & & 10 \\
\hline Ru & --- & 20 & -- & & 20 \\
\hline Sb & 1 & 20 & 0.1 & 0.1 & 15 \\
\hline Sc & 5 & 16 & -- & & 0.8 \\
\hline Se & 0.09 & 20 & 0.2 & 0.1 & 15 \\
\hline S1 & 277,200 & 72 & 0.5 & & 5 \\
\hline Sn & 6.47 & 340 & -.- & & 10 \\
\hline Sn & 40 & 164 & 0.1 & & 6 \\
\hline$s r$ & 150 & 4.8 & 0.01 & & 0.2 \\
\hline Ta & 2.1 & 640 & -- & & 50 \\
\hline To & 0.91 & 36 & $\cdots$ & & 20 \\
\hline Te & 0.0018 & 20 & 0.1 & & 20 \\
\hline TI & 4,400 & 76 & 0.25 & & 1 \\
\hline TI & 0.3 & 20 & 0.05 & & 60 \\
\hline Ta & 0.20 & 14 & --- & & 10 \\
\hline $\mathbf{u}$ & 4 & 2,000 & -- & & 75 \\
\hline$v$ & 150 & 68 & 0.2 & & 2 \\
\hline$H$ & 1 & 500 & -- & & 10 \\
\hline $\mathbf{r}$ & 28.1 & 72 & --- & & 1 \\
\hline$r$ & 2.66 & 4 & $\cdots$ & & 5 \\
\hline $2 n$ & 80 & 0.72 & 0.0005 & & 1 \\
\hline $2 r$ & 220 & 400 & -- & & 2 \\
\hline
\end{tabular}

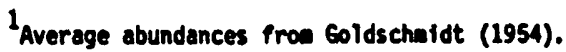

2Calculated from reciprocal sensitivity data in Perkin Elmer faAs manual such that the elewent of interest would have to be in the stated concentration in the rock to give an absorbance of 0.0044 in a 1-percent $w / v$ solution of the rock.

3 Calculated fron reciprocal sensitivity data in Perkin Elmer gFaAs nanual such that the eleaent of interest would have to be in the stated concentration in the rock to give an absorbance of 0.0044 in a 1 percent $w / v$ solution of the rock if $20 \mathrm{cal}$ of the solution was taken for analysis.

4 Experimentally deterained deteraination linits for routine analysis (Briggs and Crock, 1986, Crock, 1986, Crock and Lichte, 1982).

${ }^{5}$ Calculated by aultiplying Jarrel-Ash Corporation detection linits for pure solutions by 1,000 to account for sample dilutions necessary to achieve a low salt content solution approaching that of pure aqueous standards. These deter aination linits way be auch higher in coaplex saaples and 1 percent $w / v$ rock solutions. 
it can be seen why inductively coupled plasma-atomic emission spectroscopy (ICP-AES) has become such a popular technique. In addition to being as sensitive as FAAS for most elements, ICP-AES can determine the refractory elements at lithosphere concentrations and is also capable of simultaneous determinations. However, ICP-AES is not as versatile as FAAS in the routine analysis of high salt or organic solutions, and the determination limits for ICP-AES in these instances are generally much higher.

From table 1, we can group the elements into the following groups:

Group 1 = Elements determinable at lithosphere concentrations by FAAS.

$=\mathrm{Si}, \mathrm{Al}, \mathrm{Mg}, \mathrm{Ca}, \mathrm{Mn}, \mathrm{Na}, \mathrm{K}, \mathrm{Fe}, \mathrm{Ti}, \mathrm{Co}, \mathrm{Cr}, \mathrm{Cu}$, $\mathrm{Ni}, \mathrm{Zn}, \mathrm{Li}, \mathrm{Ba}, \mathrm{Rb}$, and $\mathrm{Sr}$.

Group 2 = Elements determinable at lithosphere concentrations by ICP-AES.

$=$ Group $1+$ rare earths + P, B, Be, Y, Sc, Sn, $\mathrm{Zr}, \mathrm{Nb}, \mathrm{Ga}$, and $\mathrm{Tl}$.

Group 3 = Elements not included in groups 1 and 2 but determinable at lithosphere concentrations by GFAAS or HGAAS.

$=\mathrm{Ag}, \mathrm{As}, \mathrm{Cd}, \mathrm{Sb}, \mathrm{Bi}, \mathrm{Mo}, \mathrm{Ge}$ and Se.

Group $4=$ Elements requiring chemical separations or concentration before being determined by one of the above techniques.

$=\mathrm{Au}, \mathrm{Pt}, \mathrm{Pd}, \mathrm{Rh}, \mathrm{Os}, \mathrm{Ru}, \mathrm{Ir}, \mathrm{Re}, \mathrm{In}, \mathrm{Hf}, \mathrm{Ta}, \mathrm{Te}$, $\mathrm{U}$, and $\mathrm{W}$.

Of those elements in Group 4, Os, Ru, Ir, Re, In, Ta, $\mathrm{Hf}$, and $\mathrm{U}$ are the only elements that, as yet, are not determined routinely at lithosphere concentrations by AAS or ICP-AES techniques at the Geologic Division laboratories.

\section{SUMMARY}

Three techniques, FAAS, GFAAS, and HGAAS, are routinely used for the determination of 35 metallic elements in geologic materials with a precision in the 1- to 10-percent relative standard deviation range. Although the techniques are relatively free of specific spectral interference overlaps, other interferences consisting of matrix, chemical, ionization, and background interferences are common and have to be controlled or eliminated for accurate determinations. A knowledge of the type of sample to be analyzed and its general composition is also very desirable to choose the proper decomposition proce- dure and analytical technique and to judge if a separation or concentration procedure is required.

Table 1 shows the approximate detection limits for the various AAS techniques as compared to the ICP-AES technique. The GFAAS and HGAAS techniques provide the lowest determination limits, and the FAAS and ICPAES techniques are more rapid and usually less prone to matrix interference.

\section{REFERENCES CITED}

Aruscavage, P. J., and Campbell, E. Y., 1979a, The determination of silver in silicate rocks by electrothermal atomic absorption spectrometry: Analytica Chimica Acta, v. 109, p. 171-175.

1979b, The determination of lead in 13 USGS standard rocks: Talanta, v. 26, p. 1052-1054.

Briggs, P. H., and Crock, J. G., 1986, Automated determination of total selenium in nonmineralized rocks, soils, and plants: U.S. Geological Survey Open File Report 86-8640, 8 p.

Crock, J. G., 1986, The determination of bismuth in geological reference materials by automated hydride generationatomic absorption spectroscopy: Analytical Letters, v. 19, p. 1367-1385.

Crock, J. G., and Lichte, F. E., 1982, An improved method for the determination of trace levels of arsenic and antimony in geological materials by automated hydride generationAtomic absorption spectroscopy: Analytica Chemica Acta, v. 144 , p. 223-233.

Goldschmidt, V. M., 1954, Geochemistry: Oxford, Oxford University Press, 730 p.

Pierce, F. D., and Brown, H. R., 1976, Inorganic interference study of atomated arsenic and selenium determination with atomic absorption spectrometry: Analytical Chemistry, v. 48 , p. $693-695$.

1977, Comparison of inorganic interferences in atomic absorption spectrometric determination of arsenic and selenium: Analytical Chemistry, v. 49, p. 1417-1428.

Robinson, J. W., 1966, Atomic absorption spectroscopy: New York, Marcel Dekker, Inc., 204 p.

Schrenk, W. G., 1975, Analytical atomic spectroscopy: New York, Plenum Press, 375 p.

Simon, F. O., Campbell, E. Y., and Aruscavage, P. J., 1977, Thallium contents of 16 USGS standard rocks: Journal of Research, U.S. Geological Survey, v. 5, no. 5, p. 579-581.

Slavin, Walter, 1968, Atomic absorption spectroscopy: New York, Interscience Publishers, John Wiley \& Sons, 307 p.

Walsh, Allen, 1955, The application of atomic spectra to chemical analysis: Spectrochimica Acta, v. 7, p. 108-119.

Welz, Bernhard, 1976, Atomic absorption spectroscopy: Weinheim, Verlag Chemie, 267 p. 


\section{CHAPTER D}

Chemical Methods of Separation for Optical Emission, Atomic Absorption Spectrometry, and Colorimetry

By S. A. WILSON, J. S. KANE, J. G. CROCK, and D. B. HATFIELD

U.S. GEOLOGICAL SURVEY BULLETIN 1770

Methods for Geochemical Analysis 



\title{
CONTENTS
}

\author{
Abstract D1 \\ Introduction D1 \\ Hydride-generation atomic absorption spectrometry D2 \\ Fire assay $\mathbf{D 3}$ \\ Ion exchange D5 \\ Mercury analysis D6 \\ Solvent extraction D8 \\ References cited D12
}

FIGURES

1. Arsenic manifold for hydride-generation atomic absorption spectrometry D4

2 Schematic representation of ion migration during ion-exchange chromatography D6

\section{TABLES}

1. Summary of commonly used separations D3

2. Detection limits and precision values for elements determined by hydride-generation atomic absorption spectrometry $\mathbf{D 3}$

3. Detection limits for the fire assay method D5

4. Ion-exchange procedures D7

5. Frequently used single-element extractions in Reston D9

6. Less frequently used single-element extractions D10

7. Multiple-element extraction methods D11

8. Cadmium extraction as a function of ligand D12

9. Percent extraction, E, for several extractions under optimum conditions 



\title{
Chemical Methods of Separation for Optical Emission, Atomic Absorption Spectrometry, and Colorimetry
}

\author{
By S. A. Wilson, J. S. Kane, J. G. Crock, and D. B. Hatfield
}

\section{Abstract}

A discussion of the several types of chemical separations that precede accurate atomic spectrometric methods of geochemical analysis is given. These include fire assay, cold-vapor mercury and hydride generation, ion-exchange chromatography, and solvent extraction. Fundamental principles of application to geochemical samples also will be presented. The problems that might be encountered in application of the methods to specific sample matrices are discussed. Means of verification of analytical accuracy and documentation of precision and detection limits are included.

\section{INTRODUCTION}

A discussion of chemical separations as a preliminary step in accurate geochemical analysis must include a variety of techniques. Among them are fire assay, coldvapor mercury and hydride generation, ion-exchange chromatography, and solvent extraction. Each has unique applications to which it is best suited and very specific requirements for success. All have a common purpose, the isolation of a single element or group of elements from the bulk sample so that the concentration of the element(s) may be determined with greatest accuracy. Additionally, all are similar in that the separation is performed, regardless of the technique employed, by moving the analyte(s), but not the bulk sample, across the phase boundary of a two-phase system. For the fire assay procedure, the two phases are the molten lead and the molten silicate glass or slag. In cold-vapor mercury and hydride generation, the two phases are the sample solution and the gaseous mixture of the generated mercury or hydride with the sweep gas used to remove it from the solution. In ion chromatographic separations, an appropriate solid resin packed in a column is one phase, and the sample solution, digest, or fusion is the second. For extractions, the sample solution and an immiscible organic solvent are the two phases used.

With the exception of the fire assay procedure, in which the sample decomposition and the separation are combined intimately with one another in the fusion step, selection of a sample dissolution procedure compatible with the subsequent separation is of critical importance. Analyte losses, either through incomplete dissolution (for example, zircon or cassiterite by simple acid digestion) or through volatilization during digestion (for example, $\mathrm{SnF}_{4}$ or $\mathrm{As}^{+3}$ in $\mathrm{HCl}$ medium), must be avoided. Residual nitrate ion from the digestion will interfere severely with iodide extraction and hydride generation and, so, must be fully removed if a nitric acid digestion is used. Extractions based on iodide ion complexation proceed with adequate yield only from a sulfuric acid medium, though most extractions can be done from the aqueous phase containing any of the common acids-nitric, hydrochloric, sulfuric, or perchloric. A full discussion of the proper pairing of sample decomposition methods with elemental separation methods is beyond the scope of this chapter, but its importance to the accuracy of the final result cannot be overemphasized. Dolezal and others (1966) is just one of many references on the subject.

Chemical separations are used to a lesser degree today than they were $20-30 \mathrm{yr}$ ago, and the specificity required of, or desired in, a separation procedure also has decreased. Originally, detection of many trace metals primarily was done by using colorimetric or potentiometric methods. Both required almost total isolation of the analyte element from all others for highest accuracy. Then the flame atomic absorption spectrometry (FAAS) technique of measurement was introduced. Because relatively few interelement effects cause error in this method, fewer separations are required than with earlier procedures. However, with the introduction of graphite furnace atomic absorption spectrometric (GFAAS) methods about $10 \mathrm{yr}$ ago, separations again were needed. Two quite severe interferences affect this method. The first is the generation in the furnace atomizer of very large background signals that cannot be corrected fully by conventional deuterium arc systems. This necessitates the extraction of cadmium, for example, from most geochemical samples, if it is to be accurately determined by GFAAS. The second interference results from the suppressing effect one element can have on the atomization of another occurring at much 
lower concentrations. In geochemical samples, lead is suppressed so severely that the method of additions cannot correct the error; therefore, separation is necessary.

With the inductively coupled plasma-atomic emission spectrometric (ICP-AES) technique, group separations taking advantage of the simultaneous multipleelement measurement potential of the instrument are feasible. Separations still are required to reduce the number of spectral interference corrections that may have to be made to obtain accurate results. Although corrections are made routinely, the total correction cannot exceed 75 percent of the apparent analyte concentration without introducing unacceptable error (Church, 1981).

To cite one example, an iron spectral overlap exists on the Cd (II) line at $214.428 \mathrm{~nm}$ which, for Fe:Cd typical of igneous rocks, always exceeds this level. Although scanning ICP-AES inștrumentation used in Menlo Park, California, allows the use of a different wavelength for determinations affected by large spectral overlaps, the direct-reader systems in the Denver, Colorado, and the Reston, Virginia, laboratories do not permit that option. However, an alternate method of measurement can be sought. As mentioned above, the alternative to ICP-AES measurement is graphite furnace measurement. For cadmium, this method also is affected by severe interferences in the absence of some separation. In some instances, it is not one large overlap, but the sum of several smaller ones that exceeds the allowable level for accurate subtraction. This was a factor in developing the ion exchange separation of the rare-earth elements from the rock matrix before ICP-AES determination (Crock and Lichte, 1982a).

Thus far, separations have been discussed without reference to preconcentration. Detection limits are sufficiently low for ICP-AES and GFAAS that preconcentration is often unnecessary. Highly refractory elements like the rare earths and elements such as the platinum group occurring generally at subpart-per-million concentrations will require preconcentration to be measurable in samples of crustal abundance levels or less. Preconcentration also is needed for those elements whose primary spectroscopic measurement lines are quite insensitive, as for the hydrideforming elements. Fire assay methods provide the very large concentration factors for the determination of the precious metals but require that very large samples, typically $15 \mathrm{~g}$, be used. Hydride generation and ionexchange can provide up to twentyfold concentration factors, without requiring more than 1-2 $\mathrm{g}$ of sample. Extraction methods cannot readily provide significant preconcentration.

Each of the separation techniques, as practiced in our laboratories, will be discussed more extensively in the sections that follow. Table 1 summarizes those used in each of the three regional centers. Differences among the centers reflect the differences in available instrumentation, in sample types routinely submitted, and in staffing levels.
Separation procedures are not required for many applications and, as they are extremely labor intensive, should be used only when necessary for the analytical task at hand.

\section{HYDRIDE-GENERATION ATOMIC ABSORPTION SPECTROMETRY}

Hydride-generation atomic absorption spectrometry (HGAAS) ${ }^{1}$ typically is used for the analysis of arsenic, antimony, selenium, bismuth, and tellurium in a variety of sample matrices. A major emphasis to date has been the analysis of these elements in natural waters (Andreae, 1977; Andreae and others, 1981; Corbin and Bosnard, 1976; Fishman and Spencer, 1977; Pyen and Fishman, 1978). Extending the technique to nonmineralized rock matrices (Briggs and Crock, 1986; Bye, 1984; Chan and Baig, 1984; Crock, 1986; Crock and Lichte, 1982b; Greenland and Campbell, 1976; Robins and Caruso, 1979; Rubeska and Hlavinkova, 1979; Sanzalone and others, 1979) has proven difficult due to the need for the appropriate decomposition and to the presence of significant chemical interferences. The method is unsuited to the analysis of mineralized samples, as the discussion of interferences will demonstrate.

The procedure for the analysis of the hydrideforming elements has four steps. In the first step, the sample is decomposed, and the element to be determined is reduced to a suitable oxidation state. For selenium and tellurium, the reduction is from VI to IV, and, for arsenic and antimony, from V to III. In step two, the sample solution is mixed with an appropriate amount of sodium borohydride. The reaction proceeds rapidly, resulting in the formation of the hydride species, as illustrated for selenium in the following equation:

$$
3 \mathrm{NaBH}_{4}+4 \mathrm{H}_{2} \mathrm{SeO}_{3} \rightarrow 4 \mathrm{H}_{2} \mathrm{Se}+3 \mathrm{H}_{3} \mathrm{BO}_{3}+3 \mathrm{NaOH} \text {. }
$$

In the third step, the gaseous product (for example, $\mathrm{H}_{2} \mathrm{Se}$ ) is stripped from the solution with argon and passed into a heated quartz furnace. The fourth step involves detection of the analyte using atomic absorption spectrometry (AAS). The spectrometer signals for calibration standards and samples are recorded on a strip chart and quantification is performed using appropriate calibration plots, regression analysis, or standard additions. Instrumentation in Denver allows the four steps to proceed in an automated continuous flow process (fig. 1), and Reston uses the nonautomated Perkin Elmer Mercury/Hydride apparatus:

\footnotetext{
${ }^{1}$ The analytical literature occasionally uses HGA-AAS to represent "heated graphite atomizer-atomic absorption spectrometry," which is synonymous to our use of GFAAS.
} 
Table 1. Summary of commonly used separations

\begin{tabular}{|c|c|c|c|c|}
\hline Center & $\begin{array}{l}\text { Solvent } \\
\text { extraction }\end{array}$ & $\begin{array}{l}\text { Hydride } \\
\text { generation }\end{array}$ & $\begin{array}{c}\text { Ion } \\
\text { exchange }\end{array}$ & $\begin{array}{l}\text { Fire } \\
\text { assay }\end{array}$ \\
\hline Reston & $\begin{array}{l}\text { Ag, As, Au, } \\
\text { Be, Br, Cd, } \\
\text { Co, Cu, Fe, } \\
\text { Mo, Nb, Mr, } \\
\text { Pb, Sb, Se, } \\
\text { Sn, Tl, H, In }\end{array}$ & $\begin{array}{l}1_{\mathrm{Hg}} \\
\text { As, Se, Te }\end{array}$ & $\begin{array}{l}\text { F } \\
\text { Br, Cl } \\
\text { SO } \\
\mathrm{Cd}_{4}, \mathrm{Mo}, \mathrm{Nb}, \\
\mathrm{Sn}, \mathrm{W}, \mathrm{Zn}\end{array}$ & $\begin{array}{l}\text { Pt, Pd, Rh } \\
\text { Au }\end{array}$ \\
\hline Denver & Au & $\begin{array}{l}1_{\mathrm{Hg}} \\
\text { As, Br, Sb. } \\
\text { Se }\end{array}$ & $\begin{array}{l}\mathrm{Br}, \mathrm{Cl}, \mathrm{F} \\
\mathrm{NO}_{3}, \mathrm{PO}_{4} \\
\mathrm{SO}_{4}\end{array}$ & $\begin{array}{l}\text { Pt, Pd, Rh } \\
A u, A g \\
\text { Ir, Ru }\end{array}$ \\
\hline Menlo & W & & $\begin{array}{l}\mathrm{Cl}, \mathrm{F} \\
\mathrm{NH}_{4}\end{array}$ & \\
\hline
\end{tabular}

${ }^{1}$ Cold-vapor generation.

The accuracy of the hydride method has been evaluated by the analysis of geochemical standards including many rock types (Crock and Lichte, 1982b; Greenland and Campbell, 1976, 1977). Information on the precision of measurement and detection limits for the method is shown in table 2 .

Two types of serious interference problems exist with the hydride method. The first is from high concentrations of certain transition and heavy metals $(\mathrm{Cu}, \mathrm{Fe}, \mathrm{Ni}$, $\mathrm{Sn}$ ). These elements can cause a 10-percent or greater reduction in signal for the hydride element if their concentration exceeds $500 \mathrm{ppm}$ in sample. The interference is based on consumption of the $\mathrm{NaBH}_{4}$ with accompanying reduction of the metal, as in equation 2 , or reoxidation of the hydride forming element so that it is in the wrong oxidation state for hydride generation, as in equation 3 ,

$$
\mathrm{Cu}^{+2} \rightarrow \mathrm{Cu}^{+} \text {or } \mathrm{Fe}^{+3} \rightarrow \mathrm{Fe}^{+2} ; 2 \mathrm{BH}_{4}^{-} \rightarrow \mathrm{H}_{2}+\mathrm{B}_{2} \mathrm{H}_{6}
$$

and

$\mathrm{Cu}^{+2} \rightarrow \mathrm{Cu}^{+}$or $\mathrm{Fe}^{+3} \rightarrow \mathrm{Fe}^{+2} ; \mathrm{As}^{+3} \rightarrow \mathrm{As}^{+5}$ or $\mathrm{Se}^{+4} \rightarrow \mathrm{Se}^{+6}$.

These interferences can be eliminated by sample dilution, but the analyte is diluted concurrently, possibly to the point that it cannot be detected. In some cases, masking agents can be used to eliminate the interference by preventing the interferent from entering into the above reactions.

The second major interference in the hydridegeneration method occurs when one hydride element is present in significantly greater concentration than another that is to be determined; for example, selenium in many geochemical samples is accompanied by much higher concentrations of arsenic. Consequently, selenium will be difficult to determine whenever arsenic in sample exceeds $1,000 \mathrm{ppm}$. Arsine will be generated preferentially, due to its greater concentration, with a dramatic suppression of the selenium hydride signal for selenium. Dilution is the only remedy.

Investigations to improve hydride-generation analytical methods are being directed toward expanding the number of elements that can be determined by this method. The successful determination of tin and lead by HGAAS has been reported (Fleming and Ide, 1976; Subramanian and Sastri, 1980). Work is also underway to develop a method for the separation of individual hydrideforming elements from one another before hydride generation. Ion exchange may be suitable.

\section{FIRE ASSAY}

The analysis of precious metals $(\mathrm{Ag}, \mathrm{Au}, \mathrm{Pd}, \mathrm{Pt}, \mathrm{Ir}$, and $\mathrm{Ru}$ ) using fire assay has been the subject of numerous investigations and publications over the years (Beamish and van Loon, 1977; Bugbee, 1940; Haffty and others, 1977; Schnepfe and Grimaldi, 1969). The traditional fire

Table 2. Detection limits and precision values for elements determined by hydride-generation atomic absorption spectrometry
Precision: relative standard deviation (RSO) in percent for cited solution concentration in parts per billion in sample (ppa)

Element Percent Parts per billition

\begin{tabular}{llll}
\hline As & 2 & 50 & 0.1 \\
Sb & 2 & 50 & 0.1 \\
Se & 2 & 50 & 0.1 \\
Bi & 1.2 & 25 & 0.03 \\
\hline
\end{tabular}




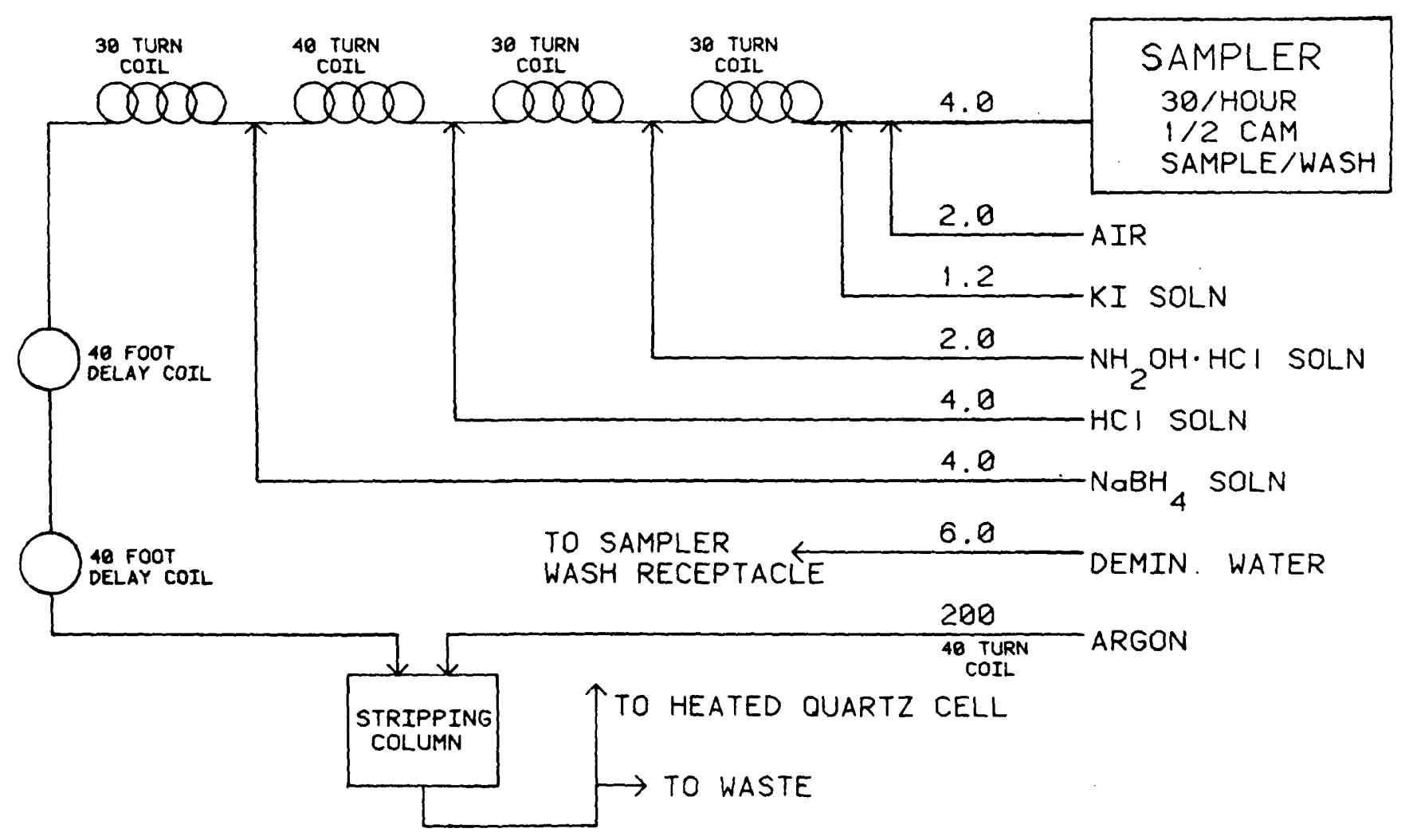

Figure 1. Arsenic manifold for hydride-generation atomic absorption spectrometry.

assay procedure still is unsurpassed in extracting trace quantities of these metals from various rock types. The method is highly selective and readily adaptable to a routine operation.

The isolation of noble metals is accomplished by mixing a sample with a predetermined flux consisting of lead oxide, sodium carbonate, silica, borax, either potassium nitrate (oxidant) or flour (reductant), and an appropriate collector. The collector is either gold, silver, or platinum, depending on which group of precious metals one is determining. The gold and platinum collectors are added to the fusion mixture in the crucible as precut wires. Silver is added as a lead-silver alloy commercially available under the name "inquarts."

During the fusion step, the crucible and its contents are heated to $1,000^{\circ} \mathrm{C}$. The lead oxide in the flux is reduced to elemental lead by the added flour or by sample constituents. The primary rock constituents fuse to form the slag. The molten lead percolates down through the slag, alloying with the precious metals on contact. After the fusion is complete ( $32 \mathrm{~min}$ ), the molten mixture is poured into a special conical steel mold. Due to the large density differences between the lead and slag, the lead settles to the bottom of the mold. After cooling, the lead button is separated physically from the slag. In the final step, called cupellation, the lead button is placed in a bone ash cup (a cupel), which is porous to lead oxide. The cupellation occurs at $840^{\circ} \mathrm{C}$, at which temperature the lead button melts. The lead is converted to lead oxide by atmospheric oxygen and is absorbed into the cupell, leaving behind the unabsorbed alloy of the precious metals and collector. This cools to a bead 1-2 mm in diameter.

In the case of platinum, palladium, and rhodium analyses, a gold collector is used. Following the fire assay, the bead is dissolved in aqua regia, and the platinum, palladium, and rhodium are analyzed by GFAAS. In the case of iridium and ruthenium, a platinum collector is used, and the metals are quantified using a direct-current arc emission technique with platinum as an internal standard. The quantification of gold is accomplished by using a silver collector followed by acid dissolution, solvent extraction, and analysis by flame AAS (FAAS). The fire assay procedure allows determination of nanogram quantities of the precious elements in a variety of rock types. Typically, the sample size is $10-15 \mathrm{~g}$. Table 3 presents the current detection limits of the method. Precision values for the procedure range from 5- to 20-percent relative standard deviation, depending on the element of interest.

Work is currently directed towards the development of two new platinum standards for use in fire assay research and as quality control standards. 
Table 3. Detection limits for the fire assay method [In parts per billion]
Eleaent

Pd

Rh

Pt

Ir

Au

Ru
Detection linit in sample

\section{ION EXCHANGE}

Ion-exchange methods are used to perform a variety of single- or multiple-element separations. Ion exchange was used extensively before the 1960's when quantification of elements relied on single-element detection by colorimetry or potentiometry. Although labor intensive and slow, ion exchange was one of the few methods available to isolate certain analytes from interfering elements (Kunin, 1949; Plummer and others, 1959; Riches, 1946) as these measurement methods required. With the introduction of FAAS, the need for ion-exchange methods declined, as discussed in the "Introduction." Recently, ion-exchange methods have come back into vogue, partly due to the advent of high-performance (high-pressure) systems capable of trace-level, multielement separations for on-line detectors (Brooks and others, 1960; Gjerde and Fritz, 1981; Pohl and Johnson, 1980; Smith and Pietrzyk, 1984) and for ICP-AES (Bolton and others, 1983; Crock and Lichte, 1982a).

Ion-exchange methods are based on the reversible exchange of ionic species between an external liquid phase (the sample solution) and a stationary ionic solid phase (the resin). The resin usually is composed of beads formed from a polystyrene divinylbenzene copolymer. Protruding from the copolymer chains are ion-exchange sites located within the bead (standard type) or fixed on the outside of the bead (new high-capacity resins). In most ion-exchange applications, the exchange sites are either sulfonic acid types $\left(\mathrm{R}^{\prime}-\mathrm{SO}_{3}{ }^{-}\right)$for cation exchange or organoamines $\left(R-R^{\prime} N^{+}\right)$for anion exchange. These sites are charged and associated with a mobile counter ion of opposite charge. This counter ion exchanges with ions

or complexes in the sample solution having charges of the same sign. It is this exchange that forms the basis for all ion-exchange work.

Positively charged analyte ions or complexes (cations) in the external liquid phase are attracted to the sulfonic acid exchange sites. Similarly, anions (negatively charged ions) will be attracted to the positive organoamine sites. The strength of these attractions is governed by the charge of the ion, its size (including its hydration sphere), and the polarizability of the ion. These factors are different for every ion or complex and will dictate which ones are held strongly or held weakly by the resin. Those ions that are strongly held will be separated successfully from the weakly held remainder. The term "selectivity coefficient" incorporates these various factors and measures, in relative terms, the binding strength of the ion-resin interaction (Lash and Hill, 1979; Plummer and others, 1959). The analyst selects the appropriate resin and complexing agent for the desired separation on the basis of these coefficients.

Two techniques are available for the separation of ions using these resins. The first is the batch approach, and the second, the column technique, is frequently called ion-exchange chromatography. The applications used at the U.S. Geological Survey utilize the column approach most extensively, and it will be the only technique discussed. Information is available on the batch method (Dean, 1969; Helfferich, 1962; Inczedy, 1966; Lash and Hill, 1979; Samuelson, 1963).

In column methods, the principles defined above apply; that is, the binding strengths of the ions are reflected in their individual selectivity coefficients, and exchange will take place between ions with charges of the same sign. These principles guide the investigator in selecting the appropriate resin and an eluent of suitable concentration and composition. The eluent contains an ion of the same sign charge as the element(s) of interest at $10-100$ times the concentration of that element or group of elements.

In column procedures, three basic steps are involved in the separation. The first is the loading of the sample solution and eluent onto the column (fig. $2 A$ ). In this example, the analytes are $A^{-}, B^{-}, C^{-2}, D^{-3}$, and the eluent is $\mathrm{E}^{-}$. In the second step (migration), the eluent ions compete with the elements being separated from the sample for exchange sites, and both undergo a series of exchange reactions (fig. $2 B$ ). During exchange, the ions with greater affinity (greater selectivity coefficient) for the resin will be retained longer on the resin, and their migration through the column will be retarded with respect to the other sample elements and eluent. Each ion will move through the column in its own discrete band. Eventually all ions, even those most retarded, will exit the 

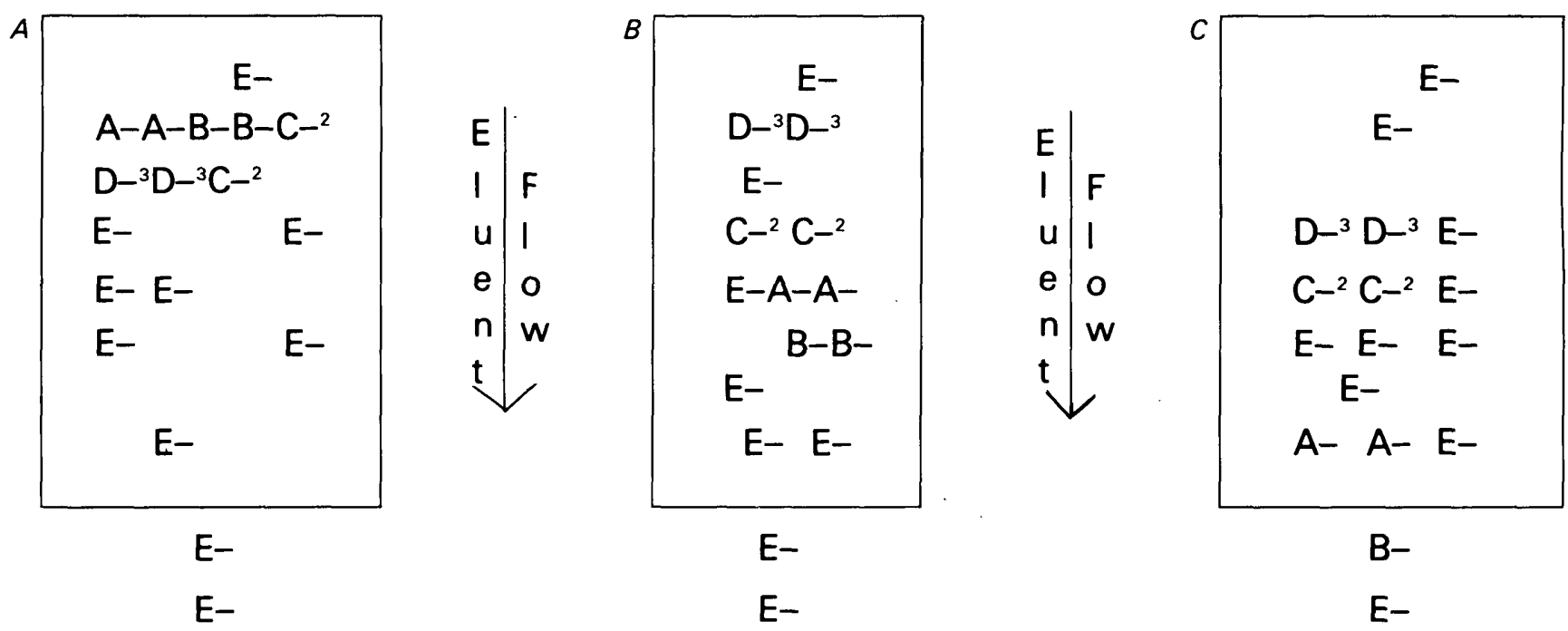

Figure 2. Schematic representation of ion migration during ion-exchange chromatography. $A$, Sample loading. $B$, Migration and exchange. $C$, Elution.

column (step three, elution) to be detected on line (fig. $2 C$ ) or collected for off-line detection. The time required for an individual ion to elute from the column is called its retention time and is characteristic of that ion under the given operating conditions. A series of single-element standards usually is injected into the system before sample analysis to determine the retention times for the elements of interest.

After the ion-exchange separation, the eluted ions can be quantified using on-line detectors (amperometric, conductimetric, or colorimetric) or off-line detectors using, for example, ICP-AES. Our applications use online detection for ammonium ion and all anion determinations and off-line detection for the determination of metals. Quantification of the analyte(s) is obtained by injecting standards of known concentrations into the column and measuring the associated signals. Sample signals then are compared to the standard signals by using established regression analysis procedures.

Detection limits, precisions, and accuracies for the ion-exchange methods used in the three centers are shown in table 4.

\section{MERCURY ANALYSIS}

Mercury continues to be of interest in a variety of geologic studies because of its environmental impact and its importance as a pathfinder element in various geologic investigations. A number of techniques have been used to determine mercury in silicate rocks. Of these techniques, cold-vapor atomic absorption spectroscopy (CVAAS) is the most widely used. In principle, it is allied closely with hydride-generation methods. The extensive use of CVAAS for the determination of mercury is due, in large part, to its simplicity, sensitivity, and the high precision which the method offers. Minor improvements to the method continue to be made, but, in general, the method used today differs little from that used in the 1960's (Bartha and Ikrenyi, 1982; Hatch and Ott, 1968; Kermoshchuk and Warner, 1981; Rains and Menis, 1972).

Mercury analyses are performed in the Reston and Denver laboratories. The two centers use different sample decomposition, and the Denver center uses a semiautomated continuous flow process similar to that for hydride analysis; however, both rely on CVAAS for final analytical determination of mercury concentration.

The mercury method used in Denver is a two-step process. In the initial step, the 0.1-g sample is decomposed with a mixture of $12 \mathrm{M}$ nitric acid and 5 percent sodium dichromate in a closed vessel for $3 \mathrm{~h}$ at $100^{\circ} \mathrm{C}$. In the second step, the sample is mixed with hydroxylamine hydrochloride and then stannous chloride to produce elemental mercury (eq 4).

The Reston procedure uses $0.1 \mathrm{~g}$ of sample that is decomposed in a sealed teflon container with a combination of perchloric, nitric, and hydrofluoric acids. Following the 3-h decomposition period, the sample is transferred to an aeration flask, stannous chloride added, and the mercury vapor generated as follows:

$$
\mathrm{Sn}^{+2}+\mathrm{Hg}^{+2} \rightarrow \mathrm{Sn}^{+4}+\mathrm{Hg}^{\circ}(\mathrm{g})
$$

The mercury vapor is swept from the aeration flask by nitrogen into a glass tube 5 in. long, which is positioned in the light path of the atomic absorption spectrometer. With the current operating conditions, the detection limit 
Table 4. Ion-exchange procedures

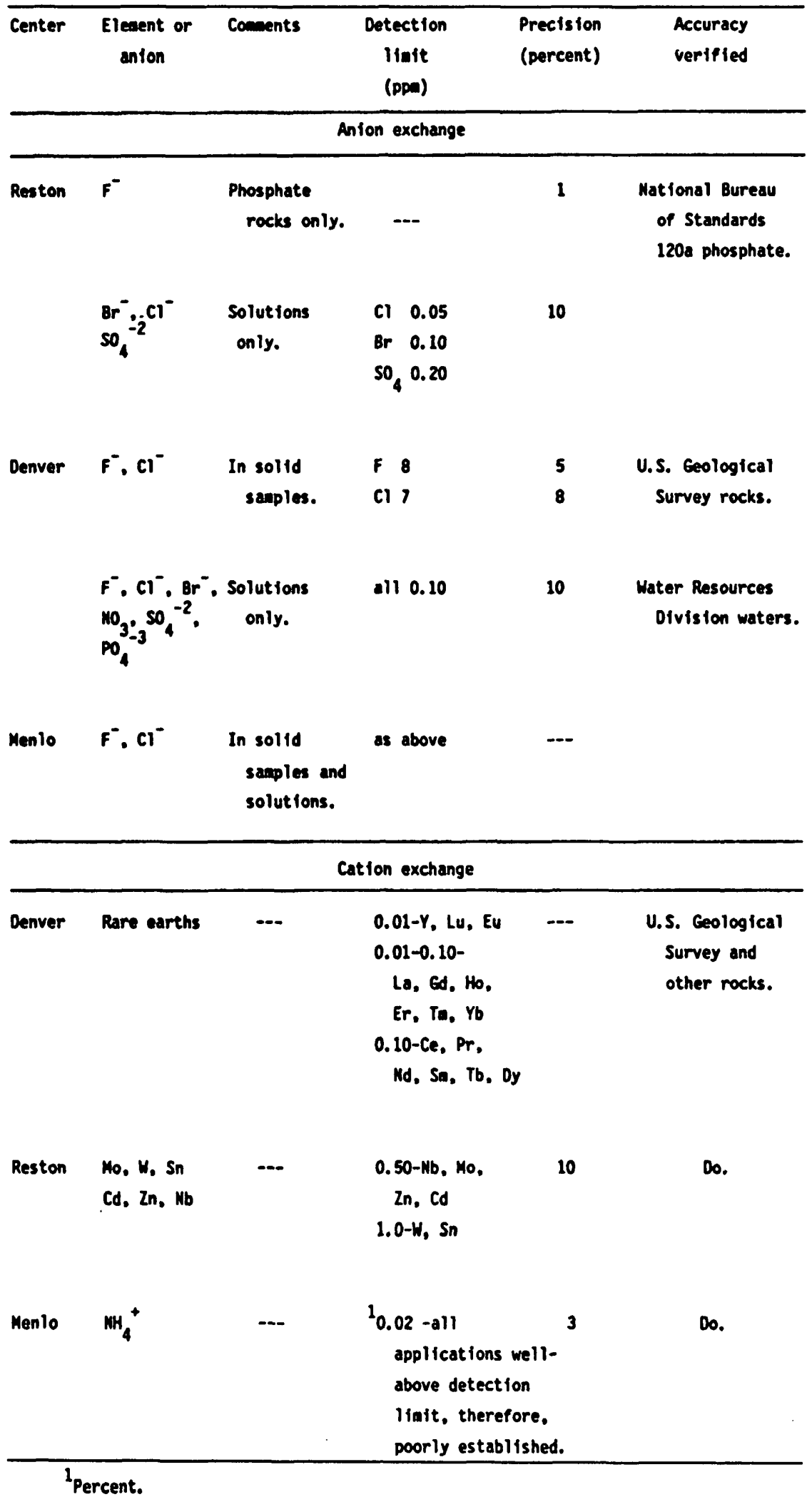


of the method is $0.02 \mathrm{ppm}$ in the sample, and the technique is linear over two orders of magnitude. The precision of the method is 10-percent relative standard deviation, based on the analysis of U.S. Geological Survey standard W-1 (0.24 ppm Hg).

\section{SOLVENT EXTRACTION}

Solvent extraction methods are used routinely in analytical chemistry to separate selected element(s) from the bulk sample matrix and to increase the sensitivity of specific instrumental methods. Preconcentration, as well as separation, with its increase in sensitivity, is needed most often only for the analysis of water samples (Kinrade and van Loon, 1974). The extractions usually are employed for the separation of trace amounts of the analyte(s) from the macro constituents but also can be used to remove a single interfering macro constituent from the sample solution. This is the case with the $\mathrm{Fe}$ extraction (Qureshi and others, 1979) cited in tables 5-7, which list all those extractions in current use for Geological Survey programs (Aruscavage, 1977; Aruscavage and Campbell, 1978, 1979a, 1981; Bothner and others, 1986; Campbell and Aruscavage, 1982; Campbell and Simon, 1978; Crock and Severson, 1980; Greenland and Campbell, 1974; Kane, 1979; Kane and others, 1982; Kane and Smith, 1981, p. 10-11; Thompson and others, 1968). The tables include brief notes on working calibration ranges for the methods, detection limits, precision, types of sample for which accuracy has been verified or for which severe problems have been identified, and instrumentation for final elemental determination.

Solvent extraction methods are based on the distribution of one or more solutes between two immiscible solvents. The most common extraction removes the analyte(s) from the aqueous phase (acid digest) to an organic solvent. The solute, which is initially soluble in both phases, will distribute itself between the two based on the differing degrees of solubility in the aqueous and organic phases. The solubility of the analyte(s) in the organic phase may be enhanced greatly through complexation reactions, which, therefore, play a major role in most trace-metal extraction schemes.

Metal ions complex readily with a large number of organic and inorganic anions. The anions are called ligands (L) when entering into complexation reactions with metal cations (M) according to equation 5 . Charges on the metal ion,

$$
\mathrm{M}+\mathrm{nL} \rightarrow \mathrm{ML}_{\mathrm{n}},
$$

the ligand, and the complex have been omitted for simplicity. Individual ligands will bind metals in the sample with different binding strengths, ligand-to-metal ratios, and complex charges (Irving and Williams, 1961; Morrison and Freiser, 1957; Ruzicka and Stary, 1968; Stary, 1964). The $\mathrm{pH}$ at which the extraction takes place will have a significant impact on the binding strengths of the ligands for the metals, as will the solvent selected for the extraction. These factors influence selection of the most appropriate ligand for a given application and determine whether the separation will be a single element or multielement. As an illustration, table 8 lists the optimum $\mathrm{pH}$ for the extraction of cadmium and the other elements that might be coextracted at that $\mathrm{pH}$ using several ligands.

The degree of extraction under any given set of conditions is given by what is called the distribution constant, $\mathrm{D}$,

$$
\mathrm{D}=\frac{\left[\mathrm{ML}_{\mathrm{n}}\right]_{\text {org }}+[\mathrm{M}]_{\text {org }}}{[\mathrm{ML}]_{\mathrm{aq}}+[\mathrm{M}]_{\mathrm{aq}}}
$$

$\left[\mathrm{ML}_{\mathrm{n}}\right]_{\mathrm{org}}$ and $\left[\mathrm{ML}_{\mathrm{n}}\right]_{\mathrm{aq}}$ refer to the metal complex concentrations in the organic and aqueous phases, respectively, and $[M]_{\mathrm{org}}$ and $[\mathrm{M}]_{\mathrm{aq}}$ refer to the concentrations of uncomplexed metal ion in the two phases. From this equation, it is seen that maximum separation will result when the metal complex formation consumes most of the analyte and the complex formed is highly soluble in the organic phase but quite insoluble in the aqueous phase, with the reverse true for the uncomplexed metal ion. When equal volumes are used for the two phases, the percent extraction, $\mathrm{E}$, of analyte is simply related to the distribution constant, $D$, from equation 6 .

$$
E=100 D /(D+1) \text {. }
$$

When $D$ is very large (greater than 100), a single extraction will transfer all analyte to the organic phase. In most procedures, $D$ is less than 100 , and multiple extractions are then needed for total quantitative transfer of the analyte. Except for instances in which preconcentration and (or) significant increase in instrumental sensitivity are the motivating forces behind the extraction, total transfer is not essential for usable extraction procedures. Constancy of $E$ for calibration solutions and for samples of highly varied matrices is the primary requirement of the method. Table 9 shows values of $E$ for a number of extractions currently used in our laboratories.

The use of solvent extraction techniques is in a continual state of flux, owing to changes in instrumentation available for final measurement and to changes in Survey program needs. Extraction and other separation methods are far more labor intensive than equivalent instrumental methods used without prior separation. Additionally, each step in the analytical procedure compounds the error because errors for the several steps are additive. The many reagents used for $\mathrm{pH}$ control, complexation reaction, and extraction can contribute to the procedural blank. For these reasons, extraction methods 
Table 5. Frequently used single-element extractions in Reston

\begin{tabular}{|c|c|c|c|c|c|c|}
\hline Element & Extraction & $\begin{array}{l}\text { Instruaent } \\
\text { for measuring }\end{array}$ & $\begin{array}{c}\text { Detection } \\
\text { linit }\end{array}$ & $\begin{array}{l}\text { Prectsion } \\
\text { percent }\end{array}$ & $\begin{array}{l}\text { Reference sanples used to } \\
\text { verify accuracy: ideal con- } \\
\text { centration range for method } \\
\text { (ppn) }\end{array}$ & other comments \\
\hline $\mathrm{Be}$ & $\begin{array}{l}\text { Acetylacetone } \\
\text { Into xylene; } \\
\text { stripped back } \\
\text { into } 3 \mathrm{HCl} \text {. }\end{array}$ & GFAAS ${ }^{2}$ & $\begin{array}{l}100 \mathrm{ppb} \\
\text { based on } \\
100 \mathrm{mg} \text {. }\end{array}$ & $3-14$ & $\begin{array}{l}\text { U.S. Geological Survey } \\
\text { standard rocks: } 0.10-12\end{array}$ & $\begin{array}{l}\text { Samples known to } \\
\text { contain inportant } \\
\text { amounts of beryl } \\
\text { require pressure } \\
\text { bonb decomposition. } \\
\text { High Al will interfere. } \\
\text { Method of additions } \\
\text { required below I ppa. }\end{array}$ \\
\hline Cd & $\begin{array}{l}\text { Dithizone } \\
\text { into xylene: } \\
\text { stripped back } \\
\text { into } 5 \text { percent } \\
\text { HCl }\end{array}$ & GFAAS & $\begin{array}{l}100 \mathrm{ppb} \\
\text { based on } \\
50 \mathrm{wt} .\end{array}$ & $5-15$ & $\begin{array}{l}\text { Mat fonal Bureau of } \\
\text { Standards coal ash } \\
\text { and U.S. Geological } \\
\text { Survey standard rocks; } \\
0.10-10\end{array}$ & \\
\hline $\mathrm{Fe}$ & $\begin{array}{l}\text { Frow } 50 \text { percent } \\
\text { HCI into KIBK }\end{array}$ & $\begin{array}{l}\text { Not for Fe } \\
\text { deternination } \\
\text { for removal of } \\
\text { Fe in prepara } \\
\text { tion for other } \\
\text { deterninations }\end{array}$ & $\begin{array}{l}\text { n: } \\
\text { of } \\
\text { a- } \\
\text { er } \\
\text { ns. }\end{array}$ & & & . \\
\hline Mo & $\begin{array}{l}\text { Zinc dithiol } \\
\text { into isoaayl } \\
\text { acotate }\end{array}$ & $\begin{array}{l}\text { Spectrophoto- } \\
\text { metric. }\end{array}$ & $\begin{array}{l}50 \mathrm{ppb} \\
\text { based on } \\
500 \mathrm{wg} \text { w. }\end{array}$ & $2-5$ & $\begin{array}{l}\text { U.S. Geological Survey } \\
\text { and other standard } \\
\text { rocks: } 0.10-40\end{array}$ & $\begin{array}{l}\text { Must ash saaples } \\
\text { high organic content. } \\
\text { Stripping not needed. } \\
\text { but organic layer must be } \\
\text { washed before weasurement } \\
\text { for complete freedon from } \\
\text { interferences. }\end{array}$ \\
\hline Nb & $\begin{array}{l}\text { Thiocyanate } \\
\text { into anyl } \\
\text { alcohol: strip } \\
\text { Into } 0.05 \text { per- } \\
\text { cent HF; develop } \\
\text { PAR COLOR }\end{array}$ & $\begin{array}{l}\text { Spectrophoto- } \\
\text { netric. }\end{array}$ & $\begin{array}{l}2 \text { ppa } \\
\text { based on } \\
250 \mathrm{w} w .\end{array}$ & $3-6.5$ & $\begin{array}{l}\text { U.S. Geological Survey } \\
\text { standard rocks; 8-185 }\end{array}$ & $\begin{array}{l}\text { Coextracted Fe can } \\
\text { interfere with color } \\
\text { developwent if } \\
\text { concentration is high. }\end{array}$ \\
\hline$S_{n}$ & $\begin{array}{l}\text { From concentrated } \\
\mathrm{H}_{2} \mathrm{SO}_{4} \text { with } \\
\text { iodide into } \\
\text { toluene }\end{array}$ & GFAAS & $\begin{array}{l}1 \text { ppo } \\
\text { based on } \\
100 \mathrm{ing} \text { wt. }\end{array}$ & $5-15$ & $\begin{array}{l}\text { U.S. Geological survey } \\
\text { standard rocks: 1-20 }\end{array}$ & $\begin{array}{l}\text { Large anounts of } \\
\text { cassiterite cannot } \\
\text { be dissolved without } \\
\text { peroxide fusion: } \\
\text { extraction after fusion } \\
\text { is possible, blank is } \\
\text { approxiaately } 50 \text { ppm. }\end{array}$ \\
\hline$\omega$ & $\begin{array}{l}\text { 2inc dithiol } \\
\text { into anyl } \\
\text { acetate; strip } \\
\text { with citrate } \\
\text { and repeat first } \\
\text { extraction }\end{array}$ & $\begin{array}{l}\text { Spectrophoto- } \\
\text { metric. }\end{array}$ & $\begin{array}{l}100 \mathrm{ppb} \\
\text { based on } \\
500 \text { wt. }\end{array}$ & 15 & $\begin{array}{l}\text { U.S. Geological Survey } \\
\text { and other standard } \\
\text { rocks } 0.20-400\end{array}$ & $\begin{array}{l}\text { with single extraction } \\
\text { coextracted interfer- } \\
\text { ing elements are not } \\
\text { adequately reasoved. } \\
\text { The element is insoluble } \\
\text { in extraction cediun } \\
\text { above } 500 \text { ppm. }\end{array}$ \\
\hline
\end{tabular}

1 Precision expressed as percent relative standard deviation. Precision is reported for values exceeding detection linit by a factor of 15 or wore; below this considerable deterioration is expected. For some elements. standard rock concentrat fons are all below 15 times the detection liait (di).

2 GfaAs: Graphite Furnace Atonic Absorption Spectrophotometry. 
Table 6. Less frequently used single-element extractions [R, Reston; D, Denver]

\begin{tabular}{|c|c|c|c|c|c|c|c|}
\hline Center & Element & Extraction & $\begin{array}{l}\text { nstruaent } \\
\text { Measuring }\end{array}$ & $\begin{array}{l}\text { Detection } \\
\text { linit } \\
\text { in parts } \\
\text { per billion }\end{array}$ & $\begin{array}{l}\text { Precision } 1 \\
\text { percent } \\
\text { (rsd) }\end{array}$ & $\begin{array}{l}\text { Reference samples used } \\
\text { to verify accuracy: } \\
\text { concentration range } \\
\text { for those saaples }\end{array}$ & Other comments \\
\hline $\mathbf{R}$ & Ag & $\begin{array}{l}\text { Diphenylthiourea } \\
\text { into butyl } \\
\text { acetate from } 20 \\
\text { percent tartaric } \\
\text { acid. }\end{array}$ & CFAAS $^{2}$ & $\begin{array}{l}10 \text { based on } \\
250 \mathrm{mg} w \text {. }\end{array}$ & $3-8$ & $\begin{array}{l}\text { U.S. Ceological Survey } \\
\text { standard rocks: } \\
7-140 \mathrm{ppb}\end{array}$ & $\begin{array}{l}\text { Organic-rich samples } \\
\text { nay lose Ag in } \\
\text { preparation before } \\
\text { extraction. }\end{array}$ \\
\hline $\mathbf{R}$ & $A u$ & $\begin{array}{l}\text { Broaide into wethyl } \\
\text { Isobutyl Ketone } \\
\text { after fire assay } \\
\text { decomposition. }\end{array}$ & FAAS $^{3}$ & $\begin{array}{l}100 \text { based on } \\
10 \text { gin wt. }\end{array}$ & & $\begin{array}{l}\text { U.S. Geological Survey } \\
\text { standard rocks: }\end{array}$ & $\begin{array}{l}\text { Unaffected by inter- } \\
\text { ference noted for } \\
\text { digestion preparation. }\end{array}$ \\
\hline D & Au & $\begin{array}{l}\text { Broaide into aethyl } \\
\text { Isobutyl Ketone } \\
\text { after } \mathrm{HBr}-\mathrm{Br}^{2} \\
\text { decomposition. }\end{array}$ & FAAS & $\begin{array}{l}100 \text { based on } \\
10 \mathrm{gn} \mathrm{wt} \text {. }\end{array}$ & 10 & $\begin{array}{l}\text { Placer material: } \\
0.05-3.0\end{array}$ & $\begin{array}{l}\text { High Fe will co--tract } \\
\text { and interfere with FAAS; } \\
\text { high Mn interference } \\
\text { can be eliminated with } \\
\text { second extraction. Sb } \\
\text { will interfere. } \\
\text { Sulfides require prior } \\
\text { ignition if organic rich. }\end{array}$ \\
\hline $\mathbf{R}$ & 81 & $\begin{array}{l}\text { Iodide Into aethyl } \\
\text { isobutyl Ketone: } \\
\text { strip with } \\
\text { ethylene-dianine- } \\
\text { tetraacetic acid } \\
\text { into aqueous phase. }\end{array}$ & GAAS & $\begin{array}{l}10 \text { based on } \\
100 \mathrm{mg} \mathrm{wt} .\end{array}$ & $9-12$ & $\begin{array}{l}\text { U.S. Geological Survey } \\
\text { standard rocks: } \\
20 \mathrm{ppb}-1.0 \mathrm{ppa}\end{array}$ & $\begin{array}{l}\text { Sone digestion procedures } \\
\text { convert Bi to unextract- } \\
\text { able chenical form. } \\
\text { Ash sanples of high- } \\
\text { organic content before } \\
\text { extraction. }\end{array}$ \\
\hline $\mathbf{R}$ & $\mathbf{P b}$ & $\begin{array}{l}\text { Diethylanonfun- } \\
\text { dithiocarbanate } \\
\text { Into xylene: strip } \\
30 \text { percent } \mathrm{HO}_{3} \text {. }\end{array}$ & EFAAS & $\begin{array}{l}500 \text { based on } \\
100 \mathrm{mg} \mathrm{wt} . \\
\text { blank is } \\
\text { liaiting } \\
\text { factor. }\end{array}$ & 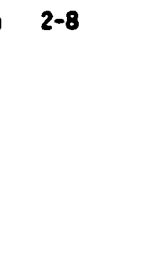 & $\begin{array}{l}\text { U.S. Eeological Survey } \\
\text { standard rocks: } \\
2.5-60 \mathrm{ppa}\end{array}$ & $\begin{array}{l}\text { Above } 10 \text { percent Fe, } \\
\text { coextraction consuaes } \\
\text { all reagent, preventing } \\
\mathrm{Pb} \text { extraction; wust } \\
\text { first rewove Fe with } \mathrm{Cl} \\
\text { extraction. }\end{array}$ \\
\hline $\mathbf{R}$ & TI & $\begin{array}{l}\text { Iodide into anyl } \\
\text { acetate. }\end{array}$ & GFAAS & $\begin{array}{l}100 \text { based on } \\
200 \text { ingt. }\end{array}$ & 8 & $\begin{array}{l}\text { U.S. Geological Survey } \\
\text { standard rocks: } \\
0.3-1.7 \text { ppa }\end{array}$ & $\begin{array}{l}\text { Only one reported value } \\
\text { exceeds } 15 \text { tines detection } \\
\text { lialt and little certainty } \\
\text { in literature for true } \\
\text { value, aaking detection of } \\
\text { reasining interferences or } \\
\text { blases probleatic. }\end{array}$ \\
\hline
\end{tabular}

1 Precision expressed as percent relative standard deviation. Precision is reported for values exceeding detection línit by a factor of 15 or more: below this considerable deterforation is expected. For some elewents, standard rock concentrations are all below 15 tiaes detection liait.

2 GFAas: Graphite Furnace Atonic Absorption Spectronetry.

3 FAAS: Flane Atonic Absorption Spectrophotonetry. 
Table 7. Multiple-element extraction methods

[D, Denver; R, Reston]

\begin{tabular}{|c|c|c|c|c|c|c|c|}
\hline Center & Element & Extraction & Instrunent & $\begin{array}{c}\text { Detection } \\
\text { Linit } \\
\text { (ppm) }\end{array}$ & $\begin{array}{l}\text { Precision }{ }^{2} \\
\text { (percent) }\end{array}$ & Reference sasples used & Other coments 1,3 \\
\hline D & $\begin{array}{l}\text { Cd, Co, } \\
\mathrm{Cu}, \mathrm{Fe}, \\
\mathrm{Mn}, \mathrm{Mr}, \\
\mathrm{Pb}, \mathrm{Zn} \\
\text { As, Sb. } \\
\text { Se }\end{array}$ & $\begin{array}{l}\text { Diethylene } \\
\text { triamine } \\
\text { pentaacetic } \\
\text { acid. } \\
\text { Iodide into } \\
\text { toluene first } \\
\text { at } 0.06 \mathrm{M} \mathrm{I} \\
\text { Sb, then at } 61 \\
\text { for As. Se. }\end{array}$ & $\begin{array}{l}\text { GFAAS } 5 \\
\text { for }\end{array}$ & $\begin{array}{l}0.100 \text { for all } \\
\text { elewents } \\
\text { based on } 100 \\
\text { ogt. }\end{array}$ & $2-15$ & $\begin{array}{l}\text { Mational Bureau of } \\
\text { Standards coal } \\
\text { standards: } 0.10- \\
6.0 \mathrm{ppm} \text {. }\end{array}$ & $\begin{array}{l}\text { Inhomogeneity of } \\
\text { saaples affects } \\
\text { imprecision; } 5^{-} \\
\text {and Cu interfere } \\
\text { seriously with } \\
\text { the extraction. }\end{array}$ \\
\hline R & $\begin{array}{l}\mathrm{Pb}, \mathrm{Cu}, \\
\mathrm{Cd} \\
\mathrm{Zn}\end{array}$ & $\begin{array}{l}\text { Diethylammonium- } \\
\text { dithiocarbanate } \\
\text { into } \mathrm{CHCl}_{3} \\
\text { from } \mathrm{MH} \mathrm{HCl} \text { and } \\
\text { then from pHB. } \\
10 \text { percent } \\
\text { citric acid } \\
\text { after first } \\
\text { removing Fe } \\
\text { (table } 5 \text { extrac }\end{array}$ & $\begin{array}{l}\text { GFAAS } \\
\text { first } \\
\text { FAAS } 6 \\
\text { t). }\end{array}$ & $\begin{array}{l}\mathrm{Pb} 1 \\
\mathrm{Cu} 1 \\
\text { Cd } 0.02 \\
\ln 1\end{array}$ & 10 & $\begin{array}{l}\text { Marine sediments } \\
\text { standards MESS-1 } \\
\text { and BCSS-1, and } \\
\text { in-house reference } \\
\text { sediments. }\end{array}$ & \\
\hline$R$ & $\begin{array}{l}\text { Co, Cu, } \\
\text { Cd, Hi, } \\
\text { Pb, Zn, } \\
\text { (BI, Ag) }\end{array}$ & $\begin{array}{l}\text { Mixed diethyl- } \\
\text { amaonilua-and } \\
\text { amaniun- } \\
\text { pyrolldine- } \\
\text { dithiocarbanate } \\
\text { into } \mathrm{CHCl}_{3} \text { afte } \\
\text { first rewoving } \\
\text { Fe (table } 5 \\
\text { extract). }\end{array}$ & $\begin{array}{l}\text { GFAAS } \\
\text { and } \\
\text { ICP-AES } \\
\text { es } \\
\text { er }\end{array}$ & $\begin{array}{l}\text { Cd } 0.06 \\
\text { Co } 1 \\
\text { Cu } 1 \\
\text { Ni } 4 \\
\text { Pb } 1 \\
\text { Zn } 5 \\
\text { Bi } 0.50 \\
\text { Ag } 0.10\end{array}$ & $\begin{array}{l}5-15 \text { for } \\
\text { rocks, at } \\
\text { to } 5 \\
\text { tines } \\
\text { detection } \\
\text { lifit. }\end{array}$ & $\begin{array}{l}\text { Standard rocks, } \\
\text { National Bureau } \\
\text { of Standards coal } \\
\text { ash, marine } \\
\text { sediaents, Hn } \\
\text { nodules, sulfide } \\
\text { ores. } \\
\text { Concentration } \\
\text { range varies } \\
\text { with eleaent, } \\
\text { In general detection } \\
\text { linit to }>0.1 \text { per- } \\
\text { cent. }\end{array}$ & $\begin{array}{l}\text { First six elenents } \\
\text { with constant } \\
\text { yfeld over the full } \\
\text { range of concentra- } \\
\text { tion for the } \\
\text { several sanple } \\
\text { types. Ag, Bi } \\
\text { extract with } \\
\text { constant yield at } \\
\text { ppa concentration } \\
\text { and above but } \\
\text { require correction } \\
\text { for low yields if } \\
\text { in the ppb range. } \\
\text { Method is blank } \\
\text { liaited for Cd. Pb } \\
\text { only; inprovement } \\
\text { of detection linit } \\
\text { would require clean } \\
\text { roon. }\end{array}$ \\
\hline
\end{tabular}

1 ppa: parts per aillition.

2 Precision expressed as percent relative standard deviation. Precision is reported for values exceeding detection limits by a factor of 15 or more: below this considerable deterioration is expected. For some elements, standard rock concentrations are all below 15 tiaes detection liait.

3 ppb: parts per billion.

4 ICP-AES: Inductively Coupled Plasma-Atomic Ealssion spectrometry.

5 GFAAS: Graphite Furnace Atoaic Absorption Spectronetry.

6 faAs: Flame Atomic Absorption Spectrometry. 
Table 8. Cadmium extraction as a function of ligand [Adapted from Ruzicka and Stary (1968, figs. 6-10)]

\begin{tabular}{|c|c|c|}
\hline Ligand/solvent & $\begin{array}{l}\text { PH range for } \\
\text { extraction of } 90 \\
\text { percent or nore }\end{array}$ & $\begin{array}{l}\text { Elewents which will } \\
\text { be } 50 \text { percent or } \\
\text { wore coextracted }\end{array}$ \\
\hline $\begin{array}{l}\text { 8-hydroxyquinoline } \\
\text { in chloroform }\end{array}$ & $5.3-10.5$ & $\begin{array}{l}\mathrm{Cu}, \mathrm{Co}, \mathrm{Ga}, \mathrm{In}, \mathrm{Pb}, \mathrm{Sc}, \mathrm{TI} \\
\text { Fe to pH } 9.5 \\
\text { Mn from pH } 6 \\
\text { Ni to pH } 9.5\end{array}$ \\
\hline $\begin{array}{l}\text { Cupferron in } \\
\text { chloroform }\end{array}$ & No extraction & \\
\hline $\begin{array}{l}\text { Dithizone in } \\
\text { carbon tetrachloride }\end{array}$ & $6-14$ & 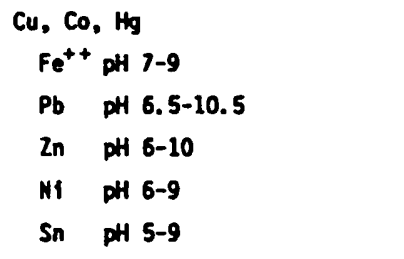 \\
\hline $\begin{array}{l}\text { Diethylamonfum } \\
\text { diethyldithio- } \\
\text { carbasate in carbon } \\
\text { tetrachloride or } \\
\text { chloroform }\end{array}$ & $1-12$ & $\begin{array}{l}\mathrm{Cu}, \mathrm{Co}, \mathrm{Hg}, \mathrm{In}, \mathrm{Pb}, \mathrm{Mf}, \mathrm{Tl}, \mathrm{Zn} \\
\mathrm{Fe}^{++}, \mathrm{Fe}^{+++} \text {PH } 2.5-11 \\
\mathrm{Mn} \text { DH 5.5-9.5 }\end{array}$ \\
\hline Thloxinate & No extraction & \\
\hline
\end{tabular}

are avoided whenever analytical requirements permit their omission. To revisit the example of cadmium so frequently cited elsewhere, zinc sulfide ores contain the element at such enriched levels relative to other geological samples that the extractions required for coal ash and rock samples are not used in the ore analysis method. Yet the agreement with literature and precision of measurement for these cadmium determinations is considerably better than that for cadmium determinations at crustal abundance levels that use the separation methods of tables 5-7.

Potentially, solvent extraction methods can be used for the determination of any metallic element in any sample matrix, rather than being limited to those elements and sample types listed in tables 5-7. Considerable method development is essential, however, to establish the analytical reliability of any new application. Such applications will continue to be developed as the need for them is recognized.

\section{REFERENCES CITED}

Andreae, M. O., 1977, Determination of arsenic species in natural waters: Analytical Chemistry, v. 49, no. 6, p. 820-823.

Andreae, M. O., Asmode, J. F., Foster, P., and Van'tdack, L., 1981, Determination of antimony (III), antimony (V), and methylantimony species in natural waters by atomic absorption spectrometry with hydride generation: Analytical Chemistry, v. 53, no. 12 , p. 1766-1771.

Aruscavage, P. J., 1977, Determination of arsenic, antimony and selenium in coal by atomic absorption spectrometry with a graphite tube: U.S. Geological Survey Journal of Research, v. 5, no. 4, p. 405-408.

Aruscavage, P. J., and Campbell, E. Y., 1978, Spectrophotometric determination of tungsten in rocks using zinc dithiol: U.S. Geological Survey Journal of Research, v. 6, no. 6, p. 697-699.

1979a, The determination of silver in silicate rocks by electrothermal atomic absorption spectrometry: Analytica Chimica Acta, v. 109, p. 171-175. 
Table 9. Percent extraction, E, for several extractions under optimum conditions

\begin{tabular}{cc}
\hline Elewent & E (percent) \\
\hline \multicolumn{2}{c}{ Single elenent } \\
\hline Be & 99 \\
\hline Bi & $85-90$ \\
Mb & $62 \pm 5$ \\
Pb & 298 \\
N & $80-90$ \\
\hline
\end{tabular}

Multielement

\begin{tabular}{cc}
\hline $\begin{array}{c}\text { Interferents for rewoval. } \\
\text { Fe }\end{array}$ & $>1$ \\
Mn & $11 \pm 3$ \\
Analytes & \\
Co & $94 \pm 2$ \\
Cu & $89 \pm 2$ \\
Pb & $100 \pm 5$ \\
Cd & $92 \pm 2$ \\
B1 & $93 \pm 1$ \\
\hline
\end{tabular}

-1979b, The determination of lead in 13 USGS standard rocks: Talanta, v. 26, p. 1052-1054.

1981, Molybdenum content of 16 U.S. Geological Survey standard rocks: Geostandards Newsletter, v. 5, no. 2, p. 171-173.

Bartha, A., and Ikrenyi, K., 1982, Multi-element analysis by inductively coupled plasma emission spectrometry inanimal diets and faeces containing chromium marker: Analytica Chimica Acta, v. 139, p. 329-332.

Beamish, F. E., and van Loon, J. C., 1977, Analysis of noble metals: New York, Academic Press, 327 p.

Bolton, Alexander, Hwang, Jack, and VanderVoet, Anthony, 1983, The determination of scandium, yttrium, and selected rare earth elements in geological materials by inductively coupled plasma optical emission spectrometry: Spectrochimica Acta, v. 38B, no. 1-2, p. 165-174.

Bothner, M. H., and others, 1986, Analysis of trace metals in bottom sediments in support of deepwater biological processes studies on the U.S. mid-Atlantic continental slope and rise: 2nd interim report, OCS Study MMS-0100, p. 57.

Briggs, P. H., and Crock, J. G., 1986, Automated determination of total selenium in rocks, soils, and plants: U.S. Geological Survey Open-File Report 86-40, 8 p.

Brooks, R. R., Ahrens, L. H., and Taylor, S. R., 1960, The determination of trace elements in silicate rocks by a combined spectrochemical-anion exchange technique: Geochimica et Cosmochimica Acta, v. 18, no. 3-4, p. 162-174.

Bugbee, E. E., 1940, A textbook of fire assaying (3rd ed.): New York, John Wiley \& Sons, 314 p.

Bye, R., 1984, Determination of selenium in zinc ore with high concentrations of lead and copper by the hydride generation-atomic absorption technique: Fresenius Zeitschrift for analytische chemie, v. 317, no. 9, p. 27-28.

Campbell, E. Y., and Aruscavage, P. J., 1982, Molybdenum and tungsten contents of five CRPG and eight ANRT geochemical reference materials: Geostandards Newsletter, v. 6, no. 2, p. 229-231.

Campbell, E. Y., and Simon, F. O., 1978, Atomic absorption determination of beryllium in geological materials by use of electrothermal atomization: Talanta, v. 25, p. 251-255.

Chan, C. Y., and Baig, M. W. A., 1984, Semi-automated method for the determination of selenium in rocks: Analytical Letters, v. 17, no. A2, p. 143-155.

Church, S. E., 1981, Multi-element analysis of fifty-four geochemical reference samples using inductively coupled plasma-atomic emission spectrometry: Geostandards Newsletter, v. 5, no. 2, p. 133-160.

Corbin, D. R., and Bosnard, W. M., 1976, Atomic absorption spectrophotometric determination of arsenic and selenium in water by hydride generation: Atomic Absorption Newsletter, v. 15 , no. 5 , p. 116-120.

Crock, J. G., 1986, The determination of bismuth in geological reference materials by automated hydride generation atomic absorption spectroscopy: Analytical Letters, v. 19, p. 1367-1385.

Crock, J. G., and Lichte, F. E., 1982a, Determination of rare earth elements in geological materials by inductively coupled argon plasma/atomic emission spectrometry: Analytical Chemistry, v. 54 , no. 8 , p. $1329-1332$.

1982b, An improved method for the determination of trace levels of arsenic and antimony in geological materials by automated hydride generation-atomic absorption spectroscopy: Analytica Chimica Acta, v. 144, p. 223-233.

Crock, J. G., and Severson, R. C., 1980, Four reference soil and rock samples for measuring element availability in the western energy regions: U.S. Geological Survey Circular $841,16 \mathrm{p}$.

Dean, J. A., 1969, Chemical separation methods: New York, D. Van Nostrand Co., 398 p.

Dolezal, Jan, Povondra, Pavel, and Sulcek, Zdenek, 1966, Decomposition techniques in inorganic analysis: New York, Elsevier Publishing Co. Inc., 224 p.

Fishman, M., and Spencer, R., 1977, Automated atomic 
absorption spectrometric determination of total arsenic in water and stream bed materials: Analytical Chemistry, v. 49 , no. 11 , p. $1599-1602$.

Fleming, H. D. and Ide, R. G., 1976, Determination of volatile hydride-forming metals in steel by atomic absorption spectrometry: Analytica Chimica Acta, v. 83, p. 67-82.

Gjerde, D. T., and Fritz, J. S., 1981, Sodium and potassium benzoate and benzoic acid as eluents for ion chromatography: Analytical Chemistry, v. 53, no. 14, p. 2324-2327.

Greenland, L. P., and Campbell, E. Y., 1974, Spectrophotometric determination of niobium in rocks: U.S. Geological Survey Journal of Research, v. 2, no. 3, p. 353-355.

1976, Rapid determination of nanogram amounts of tellurium in silicate rocks: Analytica Chimica Acta, v. 87, p. 323-328.

1977, Application of a selenium hydride-atomic absorption technique to test for homogeneity of U.S.G.S. standard rocks: U.S. Geological Survey Journal of Research, v. 5, no. 4, p. $403-404$.

Haffty, Joseph, Riley, L. B., and Goss, W. D., 1977, A manual on fire assaying and determination of the noble metals in geological materials: U.S. Geological Survey Bulletin 1443, $58 \mathrm{p}$.

Hatch, W. R., and Ott, W. L., 1968, Determination of submicrogram quantities of mercury by atomic absorption spectrophotometry: Analytical Chemistry, v. 40, no. 14, p. 2085-2087.

Helfferich, F., 1962, Ion exchange: New York, McGraw-Hill, $501 \mathrm{p}$.

Inczedy, J., 1966, Analytical applications of ion exchanges: London, Pergamon Press, 675 p.

Irving, H., and Williams, R. J. P., 1961, Liquid-liquid extraction, Chap. 31, in Treatise on analytical chemistry, pt. 1, v. 3: New York, Interscience, p. 1309-1365.

Kane, J. S., 1979, Determination of nanogram amounts of bismuth in rocks by atomic absorption spectrometry with electrothermal atomization: Analytica Chimica Acta, v. 106, p. 325-331.

Kane, J. S., Dorrzapf, A. F. Jr., and Crandell, W. B., 1982, Separation of trace metals from major and minor constituents in varied geologic matrices: Federation of Analytical Chemistry and Spectroscopy Societies, Philadelphia, $\mathbb{P a}$., Abstract 302.

Kane, J. S., and Smith, Hezekiah, 1981, Analysis of Egyptian Geological Survey and Mining Department samples by rapid rock and atomic absorption procedures: U.S. Geological Survey Open-File Report 81-991, p. 10-11.

Kermoshchuk, J. O., and Wamer, P. O., 1981, A modified method for determination of ambient particulate mercury at trace levels in suspended dust: Proceedings, Third International Conference on Heavy Metals in the Environment, p. 603-606.

Kinrade, J. D., and van Loon, J. C., 1974, Solvent extraction for use with flame atomic absorption spectrometry: Analytical Chemistry, v. 46 , no. 13 , p. $1894-1898$.

Kunin, $\mathbb{R}$., 1949, Ion exchange Analytical Chemistry, v. 21, no. 1, p. 87-96.
Lash, R. P., and Hill, C. J., 1979, Evaluation of ion chromatography for determination of selected ions in geothermal well water: Analytica Chimica Acta, v. 108, p. $405-409$.

Morrison, G. F., and Freiser, Henry, 1957, Solvent extraction in analytical chemistry: New York, John Wiley and Sons, 269 p.

Plummer, M. E. V., Lewis, C. L., and Beamish, F. E., 1959, Fire assay for platinum and palladium in ores and concentrates: Analytical Chemistry, v. 31, no. 2, p. 254-258.

Pohl, C. A., and Johnson, E. L., 1980, Ion chromatographystate of the art: Journal of Chromatographic Science, v. 18, no. 9 , p. $442-452$.

Pyen, G., and Fishman, M., 1978, Automated determination of selenium in water: Atomic Absorption Newsletter, v. 17, no. 2 , p. $47-48$.

Qureshi, M. A., Farid, M., Aziz, A., and Ejaz, M., 1979, Solvent extraction of $\mathrm{Fe}$ from steel solutions with 2-hexylpyridine prior to the determination of trace elements: Talanta, $v$. 26, p. 166-168.

Rains, T. C., and Menis, O., 1972, Determination of submicrogram amounts of mercury in standard reference materials by flameless atomic absorption spectrometry: Journal of the Association of Analytical Chemists, v. 55, no. 6, p. 1339-1344.

Riches, J. P. R., 1946, Use of synthetic resins in the estimation of trace elements: Nature, v. 158, no. 4003, p. 96.

Robins, W. B., and Caruso, J. A., 1979, Development of hydride generation methods for atomic spectroscopic analysis: Analytical Chemistry, v. 51, no. 8, p. 889A-899A.

Rubeska, Jaromir, and Hlavinkova, V., 1979, Determination of arsenic in rocks and soils by atomic absorption spectrophotometry using the MHS-1 automated hydride system: Atomic Absorption Newsletter, v. 18, no. 1, p. 5-7.

Ruzicka, Jaromir, and Stary, Jiri, 1968, Substoichiometry in radiochemical analysis: New York, Pergamon Press, 150 p.

Samuelson, O., 1963, Ion exchange separations in analytical chemistry: New York, Wiley and Sons, 474 p.

Sanzolone, R. F., Chao, T. T., and Welsh, E. P., 1979, Determination of arsenic in geological materials by electrothermal atomic-absorption spectrometry after hydride generation: Analytica Chimica Acta, v. 108, p. 357-361.

Schnepfe, M. M., and Grimaldi, F.S., 1969, Atomic absorption determination of rhodium in chromite concentrates: Talanta, v. 16, no. 11, p. 1461-1465.

Simon, F. O., Aruscavage, P. J., and Campbell, E. Y., 1977, Thallium contents of 16 USGS standard rocks: U.S. Geological Survey Journal of Research, v. 5, no. 5, p. 579-581.

Smith, R. L., and Pietrzyk, D. J., 1984, Liquid chromatographic separation of metal ions on a silica column: Analytical Chemistry, v. 56 , no. 4 , p. $610-614$.

Stary, Jiri, 1964, The solvent extraction of metal chelates: New York, Pergamon Press, 240 p.

Subramanian, K. S., and Sastri, V. S., 1980, A rapid hydrideevolution electrothermal atomic-absorption method for the determination of tin in geological materials: Talanta, $v$. 27, no. 6, p. $469-472$.

Thompson, C. E., Nakagawa, M. M., and VanSickle, G. H., 1968, Rapid analysis for gold in geologic materials: U.S. Geological Survey Professional Paper 600-B, p. B130-B132. 


\section{CHAPTER E}

\section{Analysis of Geologic Materials by Wavelength- Dispersive X-Ray Fluorescence Spectrometry}

By J. E. TAGGART, JR., J. R. LINDSAY, B. A. SCOTT, D. V. VIVIT, A. J. BARTEL, and K. C. STEWART

U.S. GEOLOGICAL SURVEY BULLETIN 1770

Methods for Geochemical Analysis 



\title{
CONTENTS
}

\author{
Abstract E1 \\ Introduction $\mathbf{E 1}$ \\ Principles of X-ray spectroscopy E1 \\ Production of X-rays E2 \\ Continuum E2 \\ Characteristic X-ray lines E2 \\ $\mathrm{X}$-ray interactions with matter E2 \\ $\mathrm{X}$-ray instrumentation $\mathbf{E 3}$ \\ $\mathrm{X}$-ray source $\mathbf{E 3}$ \\ Dispersing X-rays E4 \\ $X$-ray detectors $\mathbf{E 4}$ \\ Instrument configurations E5 \\ Current U.S. Geological Survey instrumentation E5 \\ Interferences due to matrix effects E7 \\ Absorption E8 \\ Enhancement E8 \\ Mathematical models E8 \\ Current U.S. Geological Survey practice E9 \\ Sample preparation E10 \\ Major and minor elements in rocks E10 \\ Samples that present difficulties in preparation $\mathbf{E 1 1}$ \\ Coal-ash samples E14 \\ Whole coal E14 \\ Trace-element analysis E14 \\ Precision and accuracy E15 \\ Short-term drift and stability $\mathbf{E 1 5}$ \\ Long-term drift E15 \\ Selection of calibration standards E16 \\ References cited $\mathbf{E 1 8}$
}

\section{FIGURES}

1. Flat crystal X-ray spectrometer system E3

2. In-furnace fluxer device E12

3. Fusion mold for casting fused discs for major-element analysis E13

\section{TABLES}

1. Instrument parameters for the Phillips PW1600 X-ray spectrometer E6

2. Tests of precision performed on in-house basalt standard BB-1 E7

3. Analyses in 1981 and $\mathbf{1 9 8 5}$ of seven igneous rock standards by the high-precision X-ray fluorescence majors method E17 



\title{
Analysis of Geologic Materials by Wavelength- Dispersive X-Ray Fluorescence Spectrometry
}

\author{
By J. E. Taggart, Jr., J. R. Lindsay, B. A. Scoß, D. V. Vivit, A. J. Bartel, and K. C. Siewart
}

Abstract

$X$-ray fluorescence spectromeity is applied to the determination of many major, minor, and irace elements in geological samples. $X$-ray fluorescence methods are classified on the basis of wo alternate methods of $K$-ray spectral analysis-X-ray dispersion by crystal diffraction, or wavelength-dispersive $X$-ray fluorescence, and semiconductor detectors that act as transducers in converting $X$-ray spectra to electrical signals, or energy-dispersive X-ray fluorescence (discussed in Chapier F). The former method provides comparable precision and accuracy to classical wet chemical methods for the determination of major elements in rock and mineral samples and is also applied to the determination of minor and irace elements, where the higher resolution of wavelength-dispersive instrumentation is required to reduce spectral interferences. Limitations on the method, which affect precision, accuracy, and sensitivity, arise from spectral overlap, matrix absorption and enhancement affecis, and sample inhomogeneity. Interelement and matrix effects are ireated by one or more methods including matrix matching of samples and standards, dilution, preconcentration of the element of imterest, and (or) mathematic corrections during data analysis. Two principal methods for sample preparation with the goal of presenting a representative homogeneous sample to the instrument are used routinely by the authors - the casting of flured samples into glass discs (primarily used for major-element determinations) and the pressing of finely powdered samples into pelleis for irace analysis and the analysis of small samples or where the matrix is not suitable for fusion preparation; for example, coal, the determination of volatile constituents, and so forth.

Dotailed data are presented that substantiate that the modern X-ray spectrometric techniques used by the U.S. Geological Survey are highly accurate and precise over long periods of time, highly productive, and applicable to a wide range of sample types.

\section{INTRODUCTION}

$X$-ray fluorescence $(X \mathbb{R} F)$ analysis of geologic materials has evolved over the past $25 \mathrm{yr}$ as the method preferred by the majority of earth scientists for the determination of the major and minor rock-forming elements in bulk rock and mineral separates, namely $\mathrm{Na}$, $\mathrm{Mg}$, $\mathrm{Al}, \mathrm{Si}, \mathbb{P}, \mathbb{K}, \mathrm{Ca}, \mathrm{Ti}, \mathrm{Min}$, and $\mathrm{Fe}$, because it provides higher precision and accuracy than alternative methods. The advantages of the $X R F$ method are

1. X-ray emission spectrum is simple and orderly,

2. X-ray spectra are relatively independent of chemical state,

3. X-ray excitation and absorption vary uniformly with atomic number,

4. Absorption and enhancement effects are predictable,

5. Spectral line interference is relatively infrequent,

6. Sample preparation can be nondestructive,

7. Specimen form can be solid, powder, paste, liquid, or gas,

8. High precision and accuracy can be attained, and

9. Sample preparation and analysis times can be relatively fast and are not usually labor intensive.

The disadvantages of the $X \mathbb{R F}$ method are

1. Sensitivity for low atomic number elements is frequently poor,

2. Sensitivity for low abundance levels (below part per million) is poor without preconcentration procedures,

3. Interelement effects within the sample must be recognized and corrected,

4. Standards are required, and

5. Initial cost of equipment is relatively high.

It is appropriate to first present a brief review of the fundamental theory upon which XRF analysis is based. The following sections discuss $X$-ray instrumentation and sample-preparation techniques, first in general terms and then followed by a detailed description of the specific methods used by our X-ray laboratories in Reston, Virginia, Denver, Colorado, and Menlo Park, California.

\section{PRINCIPLES OF X.RAY SPECTROSCOPY}

For the purposes of this discussion, it will be assumed that the reader is familiar with the basic concepts 
of the electronic structure of the atom. For a more complete discussion of $\mathrm{X}$-ray physics, the reader should consult Bertin (1975). This discussion will present only information necessary to explain the origin of X-ray emission lines. Extensive discussions of many aspects of the X-ray fluorescence analysis of geologic materials are contained in Fabbi (1978) and Adler (1966).

\section{Production of X-Rays}

$\mathrm{X}$-ray photons are a form of electromagnetic radiation with energies in the range of about $100 \mathrm{eV}$ to over $100 \mathrm{KeV}$. X-ray photons are produced when high-speed electrons decelerate or when electron transitions occur involving the inner orbit energy states of atoms. When an atom is irradiated by a sufficiently high energy source, an inner-shell (usually a $\mathrm{K}$ or $\mathrm{L}$ shell) electron (called a photoelectron) is ejected leaving the atom in an excited energy state. This excited energy state is not stable, and, to return to its stable ground state, a deexcitation process occurs. More than one deexcitation process may occur, and these are discussed below.

The vacancy created by the emission of an innershell electron from an atom is filled by an electron previously residing at a higher energy level. The excess energy resulting from the transition often is dissipated as electromagnetic radiation of sufficiently high energy to be called an X-ray photon. These X-ray photons have a very narrow energy bandwidth, are specific for the particular electron transitions that occur, and are characteristic of the element from which they were emitted. Thus, it is called a characteristic $\mathrm{X}$-ray line.

The X-ray photon that is emitted alternatively may interact with outer-shell electrons before it leaves the atom. When this happens, a second electron is emitted from the atom leaving it in a doubly ionized excited state. The second electron that is emitted is called an Auger electron.

In addition, the emitted inner-shell electron can interact with outer-shell electrons in the same atom, producing additional electron transitions that result in the emission of a photon having an energy in the visible or ultraviolet region of the spectrum. Because these processes occur by decreasing the energy of an electron (the electron releases its energy in the form of kinetic energy) or decelerating the electron, heat also can be given off.

\section{Continuum}

When electrons strike matter, they decelerate, giving up energy in numerous unequal increments as X-rays. When large numbers of electrons strike the target, a continuous band of $\mathrm{X}$-ray wavelengths is emitted. The continuous band is known as the continuum, the white radiation, or Bremsstrahlung and is characterized by having a continuous range of energies, limited by the energy of the striking electrons (called the Duane-Hunt limit). The continuum has a maximum intensity at approximately 1.5 times the short-wavelength limit, and the intensity gradually falls off at longer wavelengths.

\section{Characteristic X-Ray Lines}

The characteristic X-rays emitted by an atom are due to electron transitions from high energy states to lower energy states. The energy of each X-ray photon emitted as a result of a transition is equal to the difference in the energy between the initial and final energy state of the electron. Electrons are ejected from inner K, L, and M shells of an atom, which create vacancies. A vacancy can be filled by outer-shell electrons. The electron transitions that are responsible for X-ray emission are limited by a set of selection rules based on the four quantum numbers that define the energy state of each electron in the atom. Thus, each atom has several possible transitions that can occur, and each transition produces $\mathrm{X}$-rays of a unique energy. If the transition is to a $\mathrm{K}$-shell, then the X-ray is called a K X-ray, and, if the transition is to an L-shell, then it is called an L X-ray. Each X-ray is further identified by a subscript (alpha, beta, gamma, and so forth) that identifies the X-ray as originating from an electron transition between a specific energy level within the initial shell and a specific energy level within the final state. For a detailed discussion of the description and naming of X-ray lines, the reader is referred to Bertin (1975).

Moseley (1913) observed that the X-ray photons within any series (that is, $\mathrm{K}$ or $\mathrm{L} \mathrm{X-ray)} \mathrm{produced} \mathrm{by} \mathrm{these}$ transitions increase in energy with increasing atomic number of the element. A simplification of Moseley's law can be expressed as

$$
\lambda \propto 1 / Z^{2},
$$

where $\lambda$ is the wavelength of the $X$-ray photon (in nanometers) and $\mathrm{Z}$ is the atomic number of the element emitting the $\mathrm{X}$-ray photon. The energy of the $\mathrm{X}$-ray photon $(\mathrm{E})$ in kiloelectron volts $(\mathrm{KeV})$ is related to $\lambda$, in nanometers, by

$$
E_{\mathrm{keV}}=123.96 / \lambda_{\mathrm{nm}} \text {. }
$$

\section{X-Ray Interactions With Matter}

In passing through matter, $\mathrm{X}$-rays may pass through unchanged (transmission) or may undergo one of the following interactions: 
1. They may by scattered with no change in energy (Rayleigh scatter),

2. They may be scattered with a loss of energy (Compton scatter),

3. X-ray photons may be absorbed, causing an electron transition to occur with the subsequent emission of a characteristic X-ray photon (fluorescence) and a photoelectron, or

4. X-ray photons may be absorbed, followed by a radiationless energy loss.

Both types of scatter and photoelectric absorption of X-rays make up the total process for the attenuation of the incident X-ray beam. In general, the intensity of an $X$-ray beam that passes through matter obeys Lambert's Law,

$$
I=I_{0} e^{-\mu_{x}},
$$

where $I$ is the attenuated intensity, $I_{0}$ is the incident intensity, $t$ is the thickness, and $\mu_{\mathrm{x}}$ is the linear absorption coefficient. The absorption coefficient, a measure of the stopping power of a material, generally decreases with decreasing wavelength (increasing energy of the incident $\mathrm{X}$-ray beam). However, a plot of the absorption coefficient of a particular element versus energy (in kiloelectron volts) reveals that abrupt discontinuities, called absorption edges, are found in the curve. The energy of the discontinuities corresponds to the binding energy of the electron of each of the inner shells. Thus, one absorption edge is found for K X-rays; three, for L X-rays; five, for M $\mathrm{X}$-rays; and so forth.

Another absorption coefficient that will be more important in the section "Interferences Due to Matrix Effects" is the mass absorption coefficient. The mass absorption coefficient, $\mu_{m}$ (in square centimeters per gram), is related to the linear absorption coefficient by

$$
\mu_{\mathrm{m}}=\mu_{\mathrm{x}} / \rho,
$$

where $\rho$ is the density of the element (in grams per cubic centimeter) absorbing the $\mathrm{X}$-ray.

\section{X-RAY INSTRUMENTATION}

$\mathrm{XRF}$ analysis is performed by using an X-ray spectrometer. X-ray spectrometers are composed of the following major components:

1. An X-ray source,

2. A dispersion system to sort, either by wavelength or energy, the fluorescent $\mathrm{X}$-rays from the sample, and

3. A detector and measuring system to record the X-ray intensity.

$\mathrm{X}$-ray spectrometers are classified into two categorieswavelength-dispersive $\mathrm{X}$-ray fluorescence (WDXRF) spectrometers and energy-dispersive $\mathrm{X}$-ray fluorescence
(EDXRF) spectrometers (Chapter F), depending on the method used to disperse or separate the X-rays of differing energies. WDXRF spectrometers differ still further based on the type of X-ray optical system used, either flat crystal optics (conventional spectrometers usually employ this system) or focusing optics (that is, curved crystal optics) and, in some cases, semifocusing optics. The WDXRF spectrometer can be configured as a sequential spectrometer (one movable goniometer changes the crystal and its angle to measure a sequence of elements), as a simultaneous spectrometer (individual fixed goniometers for each element measured plus scanning channels), or as a hybrid instrument that can have fixed monochromators and sequential goniometers in the same instrument. A block diagram of a WDXRF spectrometer is shown in figure 1.

\section{X-Ray Source}

The $\mathrm{X}$-ray source can be an $\mathrm{X}$-ray tube, a radioactive source (such as ${ }^{55} \mathrm{Fe},{ }^{109} \mathrm{Cd}$, and so forth), or an accelerator (such as the National Light Source at Brookhaven National Laboratories, Long Island, New York). In virtually all analytical laboratories, however, the latter source is not practical. All WDXRF spectrometers in the U.S. Geological Survey use X-ray tubes as the $\mathrm{X}$-ray source. An X-ray tube is composed of a solid target, called the anode, and a filament, called the cathode (usually made from tungsten), to supply electrons and is enclosed in an evacuated glass vessel. X-ray tube targets can be made from many different metals. The target metals used in various instruments in Survey laboratories

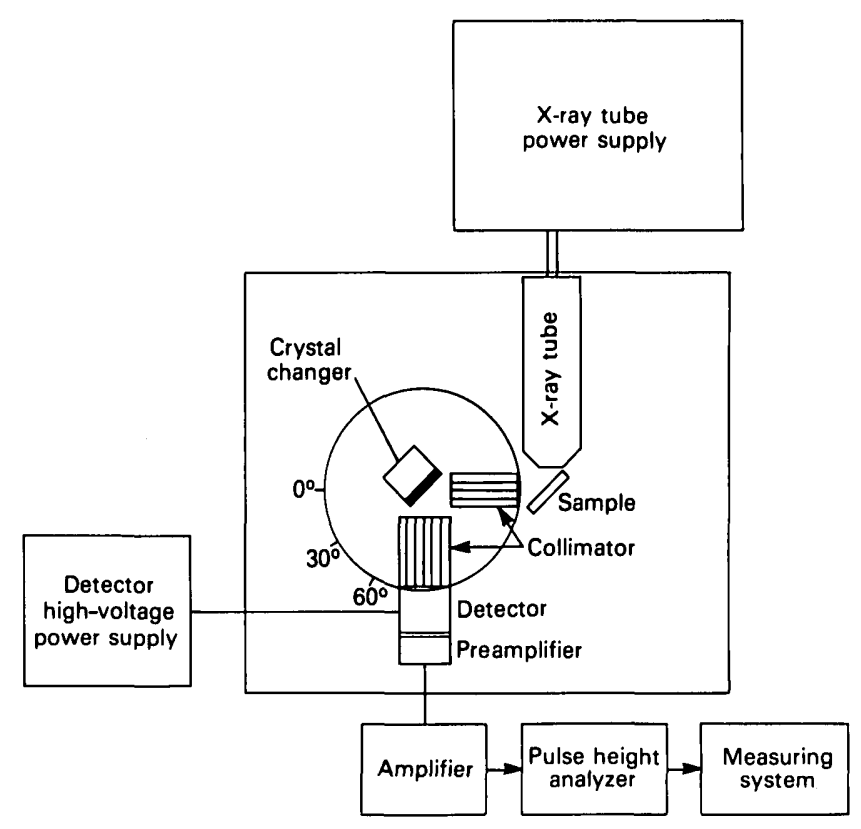

Figure 1. Flat crystal X-ray spectrometer system. 
include rhodium, chromium, platinum, tungsten, and molybdenum. A high-voltage power supply is used to maintain a high relative potential difference between the target and the fillament so that the electrons that are emitted from the filament are accelerated to a high kinetic energy before they strike the target. Upon being struck by the high-energy electrons, $\mathrm{X}$-rays are generated at the target. Because heat is produced, the target is cooled, usually by water. The X-rays exit the tube through a thin window made of material relatively transparent to $X$-rays (usually beryllium) sealed into the tube housing. The $X$-rays then can be used to irradiate a sample.

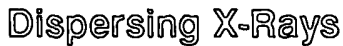

The X-rays emitted from the irradiated sample must be separated into a spectrum of wavelengths so that the characteristic $X$-rays of each element can be measured separately. Currently, two techniques are used to obtain the spectrum of X-ray lines, wavelength dispersion and energy dispersion. Energy-dispersive techniques have been developed more recently and involve the use of lithium-drifted silicon semiconductor detectors that rapidly detect the incident $X$-rays, producing electronic signals whose amplitudes are proportional to the energy of corresponding $\mathbb{X}$-rays; then, these are sorted based upon the difference in amplitude of the signal. For a complete discussion of EDXIRIF spectrometers, see Chapter $\mathbb{F}$.

WDXRF techniques wtilize the diffraction property of crystalline materials that have interatomic distances in the range from 0.14 to $1.26 \mathrm{~mm}$. Thus, a crystal is used to disperse the incidemt $X$-rays over a wide angular range so that the detector can be placed to receive only the portion of the X-rays of the element being analyzed. The most common analyzing crystals used to disperse $\mathbb{X}$-rays are lithium flluoride (the $200(\mathrm{LiF}(200))$ and $220(\mathrm{LiF}(220)$ ) lattice plames), germanium (111 plane) (Ge(111)), pentaerythritol tetrakis (hydroxymethyl) methame (PET), ammonium dihydrogen phosphate (ADP), and thallium hydrogen phthalate (TAP). Recently, mew layered symthetic microstructurall pseudocrystals (Price and others, 1985) with $d$-spacing of 2.2 up to $6.0 \mathrm{~nm}$ have been developed. They are especially efficient for dispersing X-rays from light elements (fluorine to magnesium).

For a beam of X-rays to diffract, they must exit the crystal with the X-rays in phase. By selecting a crystal with a suitable $d$-spacing and by adjusting the angle of incidence of the $X$-rays on the crystal, a characteristic X-ray line can be diffracted into a suitable detector, while most interfering $X$-rays are elliminated. This relation is described by Bragg's Law,

$$
\mathrm{m} \lambda=2 \mathrm{~d}(\sin \theta),
$$

where $n$ is an integer defining the order of the reflection, $\lambda$ is the wavelength (in manometers) of the $X$-ray dif- fracted, $\mathrm{d}$ is the distance (in nanometers) between lattice planes in the crystal, and $\theta$ is the angle of incidence of $X$-ray beam to the crystal lattice plane.

Lattice planes in crystals, especially crystals that are grown synthetically from contamination-free materials, are remarkably free from defects. However, because the lattice of a crystal cannot have its d-spacings significantly increased or decreased, WDXRF spectrometers require several different crystals to provide adequate dispersion over the entire range of $\mathrm{X}$-ray wavelengths; for example, a plate cut parallel to the (200) lattice plane of a LiF crystal has a d-spacing of $0.20135 \mathrm{~nm}$ and will diffract X-rays with wavelengths from 0.384 to $0.0351 \mathrm{~nm}$ by adjusting $\theta$. This corresponds to $\mathbb{K}_{\alpha} X$-rays for elements potassium to cerium and $\mathbb{L}_{\alpha} X$-rays for elements cadmium to lawrencium. Thus, with this crystal, an X-ray spectrometer theoretically could analyze 80 percent of the elements in the periodic table. From equations 1 and 5, however, it can be seen that the measurement of $X$-rays of elements with lower atomic number requires crystals with larger dspacings; for example, the PET crystal ( $d=0.4371 \mathrm{~nm}$ ) often is used to analyze silicon and aluminum, and the TAP crystal $(d=0.1295 \mathrm{~nm})$ or one of the multilayer analyzers is suitable for sodium and magnesium.

In conventional $X$-ray spectrometers, the analyzing crystal is a flat plate. If the analyzing crystal is bent to a radius double that of a circle (called the $\mathbb{R}$ owland circle) drawn to intersect the entrance slit of the goniometer, the crystal, and the detector, then it will focus the X-ray beam of a specific wavelength onto the detector. This optical system is referred to as Johann optics (Johanm, 1931) and is considered to be semifocusing because the X-rays striking the outer edges of the curved crystal do mot focus to the exact point as X-rays from the center of the crystal. If the crystal can be bent to a radius equall to the diameter of the IRowland circle and ground to one-half that radius, then the crystal is fully focusing, and the opticall system is of the Johansson type (Johanssom, 1933).

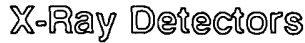

The detector is selected on the basis of its efficiency for the X-ray line being analyzed and falls into one of the following main categories: gas-filled proportional, gasflow proportional, or scintillation. Gas-filled proportional detectors contain am appropriate gas or mixture of gases. A fine wire passing through the center of the detector, but insulated from the outer body, is maintained at a high positive potential relative to the outer body. X-rays enter the detector through an opening covered by a thin window and cause the gas to ionize, forming positive ion-electron pairs. The electrons are attracted to the wire, causing a small electric current pulse to be formed whose amplitude is proportional to the energy of the incident X-ray 
photon and whose intensity is proportional to the number of events. Because it is desirable to absorb as few of the entering X-rays as possible, the windows for low-energy $\mathrm{X}$-ray detectors are constructed from materials relatively transparent to $\mathrm{X}$-radiation (made from low atomic number elements). Sealed gas-filled proportional counters are used for detecting X-rays of intermediate energy (for example, calcium $\mathbb{K}_{\alpha}$ through yttrium $\mathbb{K}_{\alpha}$ ) and usually have beryllium windows from 50 to $150 \mu \mathrm{m}$ thick. To satisfy the need to detect very low energy $X$-rays (for example, fluorine $\mathbb{K}_{\alpha}$ through potassium $\mathbb{K}_{\alpha}$ ), windows are made of very thin films, ranging from $1-\mu \mathrm{m}$ polypropylene to 6- $\mu \mathrm{m}$ Mylar. These windows are so thin that the gas slowly leaks through the film and must be replenished constantly. This type of detector is called a gas-flow proportional detector. Because a pressure differential is between the evacuated spectrometer chamber and the gas-filled proportional detector, the film must be supported by either the collimator or a nickel screen grid. The signal produced by the proportional detector is quite small and must be amplified by a preamplifier before the signal can be transmitted to the amplifier. Scintillation detectors have a crystal that, when irradiated by X-rays, emits a small amount of light. This light strikes a photocathode that emits photoelectrons which are amplified by a photodiode array. The amplitude of the electronic pulses produced by the scintillation detector is proportional to the energy of the incident $X$-ray photon, and the number of pulses is proportional to the intensity of the $\mathrm{X}$-ray beam. Scintillation detectors are used for detecting high energy X-rays (for example, above zirconium $\mathbb{K}_{\alpha}$ ).

\section{Instrument Configurations}

WDXRF spectrometers are constructed in two basic configurations known as sequential and simultaneous. A sequential spectrometer is a single-channel instrument mounted on a movable goniometer. Optimal conditions for a large range of elements are achieved by allowing variable selection of the parameters. Sequential $\mathrm{X}$-ray spectrometers are characterized by having the selection of single- or dual-target $\mathrm{X}$-ray tubes, multipleposition crystal changers, multiple collimators or attenuators, multiple detectors mounted, programmable pulse height discriminators and variable detector high-voltage power supply, and one or more movable goniometers covering the angular range from 0 to $150^{\circ} 2 \theta$. By selecting an appropriate combination of crystal, slit or collimator, detector, detector high voltage, amplifier and pulse height discriminator settings, and the appropriate angle, any element, atomic number 9 and above, can be analyzed. The primary advantage of the sequential spectrometer is that a wide range of elements may be analyzed as needs change. However, only one $X$-ray line may be detected and processed at a time, and, multiple-element determinations require that each element be analyzed sequentially.

A simultaneous X-ray spectrometer has one channel for each element to be analyzed but also may have scanning channels similar to the sequential spectrometer. Each fixed channel has a single semifocusing crystal and its own detector and preamplifier. The angular relation of the fixed channels between the entrance port, the crystal, and the detector is preset by the manufacturer to measure a particular characteristic X-ray line from one element. For each element to be determined, an additional fixed channel must be added to the instrument.

The simultaneous spectrometer can offer several advantages over the sequential spectrometer if the laboratory analyzes for a restricted group of elements and processes a large number of samples for these elements. Because the elements are measured simultaneously, the analysis time is reduced considerably. Attenuators are used to reduce the effect of detector dead time when high count rates are produced. The fact that no moving parts repeatedly need to be repositioned results in very reproducible instrument operation yielding high-precision analyses. Furthermore, the fixed channels have semifocusing crystal optics yielding significantly higher count rates with resulting improvement in counting statistics. The number of samples that can be processed also is increased greatly over a sequential spectrometer, thus making it more practical to analyze standards more frequently and to monitor the precision of the analyses.

A third type of instrument, which combines the advantages of the simultaneous and sequential spectrometers into one instrument, has been introduced that can be equipped with fixed channels and scanning gomiometers. The hybrid XRF instrument can be equipped with up to three sequential goniometers each with a six-position crystal changer, a gas-flow proportional detector, a gasflow proportional detector, and a scintillation detector. Each goniometer can scan from 0 to $152^{\circ} 2 \theta$. It also can be equipped with one goniometer and up to 10 fixed channels to simultaneously measure individual elements. Intermediate combinations are possible, with the space required for one goniometer equivalent to the space required for four or five fixed channels.

\section{Curreni U.S. Geobogican Survay unstrumentation}

Sequential, simultaneous, and hybrid $X \mathbb{R} F$ instruments are used by the Survey's analytical laboratories. The Reston laboratory has four sequential spectrometers (two Diano models XRD8300, one XRD8600, and a fully focusing spectrometer interfaced to a Diano 8000 detector-generator system). In the Denver laboratory, 
analyses are performed by using the Philips model PW1600 simultaneous X-ray spectrometer with 26 fixed channels and 2 sequential scanning channels. The Menlo Park laboratory has a Diano model XRD8600 sequential spectrometer and an Applied Research Laboratory model ARL8420 sequential spectrometer, which is the hybrid instrument, currently with one scanning goniometer and no fixed channels installed. Each instrument is different in some characteristic, and each has applications for which it is especially suited.

The Diano models XRD8300 and XRD8600 in Reston are used for the determination of chlorine and phosphorus in raw coal and for the $\mathbf{1 1}$ major elements in rocks and coal ash. The sodium and magnesium also are determined on the fully focusing spectrometer, which was designed and constructed at the Survey (Lindsay and others, 1978) and is especially good for measurement of the low-energy $\mathrm{X}$-rays emitted from sodium and magnesium. It has only one crystal (TAP) and one flow proportional detector; the crystal is curved so that the $\mathrm{X}$-rays of a particular wavelength that strike it are focused on a slit in front of the detector. Pure methane is used for the gas-flow proportional detector because it eliminates a potential interference on the magnesium determination caused by the presence of calcium.

The Denver laboratory uses the Philips PW1600 simultaneous X-ray spectrometer to perform the highprecision major-element analysis of rocks and mineral separates. Since 1979, the majority of the major-element analyses performed on crustal rock samples within the Geologic Division have been done on the Philips PW1600 simultaneous WDXRF spectrometer with an end-window rhodium-target X-ray tube operating at $35 \mathrm{kV}$ and 60 $\mathrm{mA}$. This permits quantitative measurements of elements as light as sodium. The various parameters for each channel are given in table 1.

To ensure maximum instrument stability, the Philips PW1600 is kept at operating conditions at all times. Each day, the calibration is checked and, if necessary, updated. To monitor stability during the time that analyses are

Table 1. Instrument parameters for the Phillips PW1600 X-ray spectrometer

[Line $=K_{\alpha} ; \mu \mathrm{m}$, micrometers; TLAP, thallium hydrogen phthalate; PET, pentaerythritol tetrakis (hydroxymethyl) methane; InSb, indium antimonide; GE, germanium (111); LF, litthium fluoride (200);P10 gas, 90 percent argon +10 percent methane]

\begin{tabular}{|c|c|c|c|}
\hline Element & Crystal & Detector gas & Window \\
\hline Ma & Sendfocused TLAP & Flowcounter, P10 & $1-\infty \omega$ polypropylene. \\
\hline Mg & --dom & --do-- & D. \\
\hline AI & Senifocused PET & --do-- & Do. \\
\hline si & Senifocused InSb & --do-- & Do. \\
\hline $\mathbf{p}$ & Senifocused EE & $--d 0--$ & Do. \\
\hline $\mathbf{K}$ & Senifocused PET & --do-- & Do. \\
\hline ca & Senifocused LIF & Sealed argon & 50-nn berylliua. \\
\hline TI & --do-- & --do-- & Do. \\
\hline Mn & -- do-- & --do-- & Do. \\
\hline Fe & --do-- & --do-- & Do. \\
\hline
\end{tabular}


being performed, every tenth sample is a disc of known composition. The figures given in columns 5 and 6 of table 2 are actual examples of the data collected on discs of BB-1 (U.S. Geological Survey internal standard reference basalt) counted every tenth sample.

The Diano XRD8600 X-ray spectrometer has been used for the determination of the major and minor elements in rocks in the Menlo Park laboratory. The instrument is quite similar to the instrument in Reston and is operated under the same parameters. Recently, the major- and minor-element procedures were transferred to the new ARL8420 X-ray spectrometer. The ARL8420 can be operated at higher X-ray tube power than the Diano instruments and has much better sensitivity for measurement of the light elements. The instrument automatically compensates for dead-time and pulse-amplitude shifts at very high count rates so that these problems are no longer important. The Diano XRD8600 still is used to determine selected trace elements in rocks.

\section{INTERFERENCES DUE TO MATRIX EFFECTS}

A sample that is composed of more than two elements is considered to be a complex sample. Whenever a complex sample is analyzed, the effect of the other constituents on the analyte $\mathrm{X}$-ray line may be significant enough to cause errors in the analysis. These effects generally are referred to as matrix effects. Matrix effects can be divided into two categories - mechanical and physical. Mechanical matrix effects are related to the preparation of the sample for analysis and result from inhomogeneous samples or insufficient sample to represent an infinite thickness for the elements in the sample. The physical matrix effects are the result of absorption of the fluorescent X-rays from the analyte element by the matrix elements or enhancement of the analyte $\mathrm{X}$-ray line due to secondary fluorescence from one or more of the matrix

Table 2. Tests of precision performed on in-house basalt standard BB-1

\begin{tabular}{|c|c|c|c|c|c|c|}
\hline$c_{0}$ & $\begin{array}{l}\text { (1) } \\
\text { Sample } \\
\text { Composition } \\
\text { (percent) }\end{array}$ & $\begin{array}{l}\text { (2) } \\
\text { Contribution } \\
\text { of error by } \\
\text { counting } \\
\text { statistics }\end{array}$ & $\begin{array}{l}\text { (3) } \\
\text { Precision, one } \\
\text { sample counted } 50 \\
\text { times in } 1.5 \mathrm{~h} \\
\text { (standard deviation) }\end{array}$ & $\begin{array}{l}\text { (4) } \\
49 \text { separately pre- } \\
\text { pared discs counted } \\
\text { once each in } 1.5 \mathrm{~h} \\
\text { (standard deviation) }\end{array}$ & $\begin{array}{l}\text { (5) } \\
1 \text { dise counted } \\
62 \text { times from } \\
7-81 \text { to } 12-81 \\
\text { (standard deviation) }\end{array}$ & $\begin{array}{c}\text { (6) } \\
1 \text { disc counted } \\
62 \text { times from } \\
1-85 \text { to 5-85 } \\
\text { (standard deviation) }\end{array}$ \\
\hline $\mathrm{SIO}_{2}$ & 52.8 & \pm 0.04 & 0.04 & 0.13 & 0.19 & 0.09 \\
\hline $\mathrm{Al}_{2} \mathrm{O}_{3}$ & 16.7 & \pm 0.03 & 0.02 & 0.07 & 0.05 & 0.05 \\
\hline $\mathrm{LFe}_{2} \mathrm{O}_{3}$ & 9.35 & \pm 0.01 & 0.01 & 0.03 & 0.02 & 0.01 \\
\hline $\mathrm{MgO}$ & 3.54 & \pm 0.02 & 0.02 & 0.02 & 0.06 & 0.04 \\
\hline CaO & 6.50 & \pm 0.01 & 0.01 & 0.02 & 0.02 & 0.02 \\
\hline $\mathrm{Na}_{2} \mathrm{O}$ & 3.20 & \pm 0.03 & 0.03 & 0.09 & 0.07 & 0.04 \\
\hline$k_{2} O$ & 4. 38 & \pm 0.01 & 0.01 & 0.01 & 0.03 & 0.01 \\
\hline $\mathrm{THO}_{2}$ & 0.88 & \pm 0.01 & 0.01 & 0.01 & 0.01 & 0.01 \\
\hline $\mathrm{P}_{2} \mathrm{O}_{5}$ & 0.60 & \pm 0.01 & 0.01 & 0.01 & 0.01 & 0.01 \\
\hline MnO & 0.18 & \pm 0.01 & 0.01 & 0.01 & 0.01 & $<0.01$ \\
\hline
\end{tabular}

${ }^{1}$ Total Fe as $\mathrm{Fe}_{2} \mathrm{O}_{3}$ 
elements. Mechanical matrix effects will be discussed later in the section "Sample Preparation." Matrix effects due to absorption and enhancement, along with a few of the common mathematical procedures to correct for these effects, will be discussed below.

\section{Absorption}

The absorption characteristics of a sample vary with the wavelength of the $X$-ray and with the composition of the sample. For each characteristic $X$-ray, the mass absorption coefficient of a sample $\left(\mu_{s}\right)$ is equal to the sum of the individual mass absorption coefficients of each constituent element multiplied by its corresponding weight fraction

$$
\mu_{s}=\Sigma \mu_{i} W_{i},
$$

where $\mu_{\mathrm{i}}$ is the mass absorption coefficient of each matrix element on each characteristic $X$-ray wavelength and $W_{i}$ is the corresponding weight fraction. Thus, the total mass absorption coefficient of a sample can be considerably different from that of other samples because of gross differences in concentrations of the matrix elements. A basalt and an andesite may be significantly different in composition to a geologist, but, in terms of matrix effects, their composition is quite similar. If, however, a similar sample of andesite contains a few percent of copper, zinc, and lead mineralization, then a significant matrix effect occurs for which a correction must be made, otherwise the accuracy of the analysis for the major elements is not known.

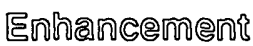

Secondary fluorescence has the opposite effect from absorption. When an X-ray photon is absorbed, it causes electron transitions to occur as described above ("Production of X-Rays"). The X-rays generated by the elements in the sample are called secondary, or fluorescent, X-rays [X-rays from the primary excitation source (that is, the X-ray tube) are called primary X-rays].

Although the X-ray flux from the X-ray tube is approsimately constant, the X-rays produced through secondary fluorescence vary with changes in the composition of the sample. As the fluorescent X-rays pass through the sample, some of them can be absorbed by other elements in the sample. The absorbed X-rays cause electron transitions to occur that result in additional fluorescent characteristic X-rays of the matrix element being emitted. The number of these fluorescent $X$-ray photons is dependent on the concentration of the element causing the secondary emission (and is in addition to the number of $\mathrm{X}$-rays emitted from excitation by the $\mathrm{X}$-ray tube) and thus varies from sample to sample with changes in the concentration of that element (an enhancement effect). When the concentration of an element is calculated on the basis of the intensity of its characteristic fluorescent X-ray intensity, the effect of this enhancement must be taken into account.

Before X-ray spectrometers were automated with computers, the task of correcting $\mathrm{X}$-ray intensity measurements for absorption and enhancement effects was quite time consuming and tedious. Often, it was easier to alter the sample in some way to minimize these effects. Many procedures were introduced that ranged from chemical alteration of the sample by dissolution and isolation of the element of interest (Rose and Cuttitta, 1968a, b; Cuttitta and Rose, 1968) to using high dilution of the sample with a solid diluent followed by fusion [for example, Claisse (1957) used 1:100 sample:borax] to the addition of a material that had a very high absorption cross section for the X-rays to be measured [for example, $R$ $(1962,1963)$ used 1:1:8 sample: $\left.\mathrm{La}_{2} \mathrm{O}_{3}: \mathrm{Li}_{2} \mathbb{B}_{4} \mathrm{O}_{7}\right]$. Although these procedures improved the quality of the analytical data by diminishing the magnitude of matrix effects, they frequently reduced the count rate, required additional steps in the sample preparation procedure, and, hence, gave more opportunity for error in the analysis.

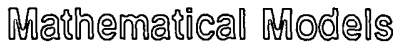

Since the early to mid-1970's, most commercial X-ray spectrometers have been automated with minicomputers, and more sophisticated mathematical correction procedures became more readily available. The current trend in XRF analysis is to employ the best samplepreparation procedure to remove all mechanical matrix effects and to use one of the mathematical correction procedures to correct for the absorption and enhancement matrix effects.

Over the years, mumerous mathematical models have been proposed for the treatment of interelement matrix effects in $X \mathbb{R} F$ analysis. For this discussion, we will mention only the six models that are currently available for use with the X-ray spectrometers in the Geologic Division laboratories. [For a more complete discussion see Tertian and Claisse (1982) and Lachance (1983).] The six models are of two types-those that are concentration dependent and those that are intensity dependent. The concentration-dependent models include the Lachance-Traill model (Lachance and Traill, 1966), the Claisse-Quintin model (Claisse and Quintim, 1967), the deJongh modell (deJongh, 1973), and the RasberryHeinrich model (Rasberry and Heinrich, 1974). The intensity-dependent models are the ILucas-Tooth and Pyne model (Lucas-Tooth and Pyne, 1964) and the multiple 
linear regression models (Alley and Myers, 1965; Mitchell and Hopper, 1966). The equations for each of these models are given below.

Lachance-Traill model

$$
C_{i}=I_{i} / I_{100 i}\left[1+\sum_{j=1}^{n}\left(a_{i j} C_{j}\right)\right](j \neq i)
$$

Rasberry-Heinrich model

$$
(C / R)_{i}=1+\sum_{j=1}^{n} a_{i j} C_{j}+\sum_{j=1}^{n} B_{i j} C_{j} /\left(1+C_{i}\right)
$$

Claisse-Quintin model

$$
(C / R)_{i}=1+\underset{i \neq j}{\sum_{i j} C_{j}}+\underset{i \neq j \neq k}{\sum_{j}} B_{i j k} C_{j} C_{k}
$$

deJongh model

$$
\mathrm{C}_{\mathrm{i}}=\mathrm{C}_{\mathrm{i}}^{*}\left(\mathrm{I}_{\mathrm{i}} / \mathrm{I}_{\mathrm{i}}^{*}\right)\left[1+\Sigma \mathrm{a}_{\mathrm{ij}} \mathrm{C}_{\mathrm{i}}-\Sigma \mathrm{a}_{\mathrm{ij}} \mathrm{C}_{\mathrm{j}}^{*}\right]
$$

Lucas-Tooth and Pyne model

$$
C_{i}=k_{i}+I_{i}\left[a_{i}+\sum a_{i i} I_{i}+\sum_{j=1}^{n}\left(a_{i j} I_{j}\right)\right]
$$

Multiple linear regression

where

$$
\begin{aligned}
& C_{i}=k_{o i}+I_{i}\left(a_{i}+\Sigma a_{i j} I_{j}\right) \\
& C_{i}=k_{o i}+a_{i} I_{i}+\Sigma a_{i j} I_{j} \\
& C_{i}=k_{o i}+a_{1} I_{i}\left(k_{2}+\Sigma a_{i j} I_{j}\right)
\end{aligned}
$$

$$
\begin{aligned}
& i=\text { analyte element, } \\
& \mathrm{j}=\text { matrix element, } \\
& \mathbf{k}=\text { matrix element, } \\
& \mathrm{n}=\text { number of elements in the matrix, } \\
& \mathrm{C}_{\mathrm{i}}=\text { concentration of the analyte element, } \\
& C_{j}=\text { concentration of each element in the } \\
& \text { matrix, } \\
& C_{k}=\text { concentration of each element in the } \\
& \text { matrix, } \\
& I_{i}=\text { analyte } X \text {-ray line intensity, } \\
& I_{100_{i}}=X \text {-ray line intensity for pure analyte ele- } \\
& \text { ment, } \\
& I_{j}=X \text {-ray line intensity of each element in the } \\
& \text { matrix, } \\
& a_{i j}=\text { influence coefficient of each matrix ele- } \\
& \text { ment } j \text { on the analyte element, } \\
& \mathrm{a}_{\mathrm{i}} \text { and } \mathbf{k}_{\mathrm{i}}=\text { constants, } \\
& k_{o i} \text { and } k_{2}=\text { constants, } \\
& a_{i i}=\text { influence coefficient for the analyte ele- } \\
& \text { ment on itself, } \\
& R=\text { ratio of intensity of analyte } X \text {-ray line to } \\
& \text { intensity of pure analyte element, } \\
& B_{i j}=\text { influence coefficient due to fluorescence, } \\
& B_{i j k}=\text { influence coefficient for secondary effect } \\
& \text { of matrix elements, }
\end{aligned}
$$

$$
\begin{gathered}
\mathrm{C}_{\mathrm{i}}^{*}=\begin{array}{c}
\text { concentration of the analyte element in a } \\
\text { standard of similar composition, }
\end{array} \\
\mathrm{I}_{\mathrm{i}}^{*}=\begin{array}{c}
\text { intensity of } \mathrm{X} \text {-ray line of analyte element } \\
\text { in a standard of similar composition, }
\end{array} \\
\mathrm{C}_{\mathrm{j}}^{*}=\begin{array}{c}
\text { concentration of each matrix element in a } \\
\text { standard of similar composition. }
\end{array}
\end{gathered}
$$

\section{Current U.S. Geological Survey Practice}

The high-precision major-element analysis procedure performed at the Survey in Denver on a Philips PW1600 uses the deJongh model (deJongh, 1973). The influence coefficients for this model are calculated by the Philips Electronic Instrument Company for the user on the basis of the user's instrument configuration and the average composition of the matrix to be analyzed. The range of samples that can be analyzed with a single calibration using this model is extended by igniting the sample at $925^{\circ} \mathrm{C}$ to obtain loss on ignition (LOI) and then fusing the ignited sample with lithium tetraborate using a 1:10 sample:flux dilution (see "Sample PreparationMajor and Minor Elements in Rocks"). The average sample composition chosen for determination of the influence coefficients was given as 91 percent lithium tetraborate and 9 percent AGV-1 (an andesite standard) as representative of the majority of samples analyzed. Material lost on ignition is compensated for by assigning LOI as an element with its influence coefficient equal to zero and adjusting all other influence coefficients accordingly. At a later time, LOI is added to the report for reference purposes. If a sample is significantly different chemically from the composition of AGV-1, especially if the sample contains a substantial amount of an element not determined as part of the analysis, then no correction for that element on the other elements being determined can be made, and their results will be biased. For this reason, samples with more than 5 percent of an interferring element (especially heavy absorbers) are not reported as quantitative results.

The Diano model XRD8600 in the Menlo Park laboratory uses three multiple linear regression models to correct for absorption, enhancement, line interference, and background effects (eqs 12-14). Each equation is used to correct for a specific effect or a combination of matrix effects (eq 14), and a measuring program employs the necessary equation to handle expected problems. When a multiple-regression equation is used to compensate for matrix effects, the number of effecting variables (in this case, elements) are kept to a minimum. As the number of variables increases, the number of standards required for the calibration increases by approximately $2 n^{2}+1$ ( $n$ is the number of effecting elements). The generation of influence coefficients with multiple regression assumes that the concentration values input are 
distributed randomly to reduce the possibility of accidental correlations.

Equation 12 produces influence coefficients where absorption and enhancement effects predominate. The absorption or enhancement effects will change the slope of the intensity versus concentration line. Enhancement will result in an increase in the intensity and will have a negative coefficient. Absorption can result in either an increase or a decrease in the intensity of the measured $\mathrm{X}$-ray line depending on the composition of the sample. Thus, absorption can result in either a positive or a negative influence coefficient. If the absorption of the effecting element is less than the self absorption of the analyte, then the influence coefficient will be negative, and, if it is greater than the self absorption of the analyte, then it will be positive.

Equation 13 is used when a line interference exists or when large variations in the background cause significant variations in the intensity of the analyte $\mathrm{X}$-ray line. $\mathrm{A}$ line interference or background variation results in a parallel shift of the intensity versus concentration line (change in the intercept without a change in the slope). The influence coefficient generated is always negative.

The last regression equation (eq 14) combines the absorption-enhancement and line interference or background effects into one regression equation when both effects are of significance in a sample. To use this equation, the influence coefficient for the line interference or background effect $\left(\mathbb{K}_{2}\right)$ must be determined by an independent measurement and the coefficient input into equation 14.

The ARL8\$20 has all the above-mentioned models available except the deJongh and the Claisse-Quintin. The choice of model depends on the type of analysis being performed. For major- and minor-element analysis on the ARL8420, using high dilution followed by fusion for sample preparation, the Lachance-Traill model is used to correct for matrix effects. Dead-time and $\mathrm{X}$-ray line interference corrections must be made before applying a correction model. The Lucas-Tooth and Pyne model, when used for the matrix correction for major- and minor-element analysis of geologic materials, gives results comparable to the Lachance-Traill model. The RasberryHeinrich model has been applied successfully to the analysis of stainless steel samples (Rasberry and Heinrich, 1974) but has proven to be of limited value for the analysis of geologic materials (Lindsay and others, 1982).

The Philips PW1600 simultaneous X-ray spectrometer has the Lachance-Traill (eq 7), Rasberry-Heinrich (eq 8), deJongh (eq 10), and Lucas-Tooth and Pyne (eq 11) models available for matrix corrections. During the first year (1979) of operation of the Phillips PW1600, the Lucas-Tooth and Pyne model was used. Since 1980, however, the deJongh model has been used for the high- precision $X \mathbb{R F}$ analysis of the major- and minor-elements in rocks.

The Diano XRD8300 and XRD8600 in the Reston laboratory, however, have been modified to run a specially developed computer automation and data processing program called X-SOFT (Spinella and Lindsay, 1981). The series of programs can perform the interelement corrections using any of the models listed above by a series of transforms that, when computed sequentially, are identical to the model. In addition, linear regression equations, similar to those used in the Menlo Park facility, are also available.

\section{SARPLE PREPARATION}

\section{Ahajor and Mhinor Elements in Rocks}

With the improvement in modern X-ray spectrometers, the limiting factor on quality of analysis has come to be sample preparation and presentation to the instrument. As a result, a great deal of development work has been done in the field of sample preparation; not too surprisingly, the analyst's personal preference, cost, through-put, and so forth also must be considered. Within the Survey, in terms of number of samples, the major- and minorelement technique largely has been performed on the Philips PW1600 in the Denver laboratory by using the following preparation technique, which also has been incorporated by the other X-ray centers, but on a smaller scale.

Samples are received as a homogeneous, -100 mesh powder prepared in the grinding room. Molded glass discs were selected as the method of preparation for XRF major-element analysis because of the relative stability of glass discs, and the necessity of eliminating possible contamination during the grinding and making of briquets from a fusion bead using the technique of Fabbi (1972). LOI is an important and integral part of the majorelement analysis of geologic samples. An 0.800 -g portion of the powdered sample is ignited in a tared 95-percent platinum-5-percent gold alloy (hereafter referred to as Pt-Au) crucible at $925^{\circ} \mathrm{C}$ for $45 \mathrm{~min}$. The crucible then is cooled in a desiccator and weighed. The weight loss is reported as percent $\mathbb{L O I}$ and includes the loss of volatile substances, such as $\mathrm{H}_{2} \mathrm{O}, \mathrm{CO}_{2}$, organic carbon, and less common constituents such as $\mathbb{H g}$, graphite, Se, and so forth. The $\mathbb{L O I}$ also would include any weight gain from stoichiometric oxygen in the oxidation of $\mathrm{Fe}^{2+}$ to $\mathrm{Fe}^{3+}$, which would serve to offset some of the weight loss. Some clay minerals and high-silica materials, however, will melt partially during the $925^{\circ} \mathrm{C}$ ignition, preventing a good mechanical mixture of the sample with the flux, resulting in an inhomogeneous glass disc. In these cases, a second $0.800-\mathrm{g}$ portion of the unignited sample can be mixed with the flux and used to prepare the glass disc, retaining the 
LOI value from the ignition of the first portion. Under routine conditions, however, the sample does not melt, and a small portion (about one-tenth of the total weight) of an 8-g charge of lithium tetraborate flux (some research projects employ different flux mixtures; see for example, Baker, 1982) is added to each crucible, and an agate pestle is used to pulverize and mix the ignited sample and flux together. The remainder of the lithium tetraborate is dumped into the crucible and thoroughly mixed with the sample. To serve as a nonwetting agent, $250 \mu \mathrm{L}$ of a 50 percent solution of $\mathrm{LiBr}$ (made by neutralizing concentrated $\mathrm{HBr}$ with $\mathrm{Li}_{2} \mathrm{CO}_{3}$, filtering, and diluting 1:1 with $\mathrm{H}_{2} \mathrm{O}$ ) is added to the contents of each crucible. This prevents the finished disc from sticking to the mold. Seven crucibles, each containing a sample, and seven empty molds are loaded into an automatic fluxer (Taggart and Wahlberg, 1980a). The fluxer, crucibles, and molds are picked up as a unit and placed in a muffle furnace at $1120^{\circ} \mathrm{C}$ for $40 \mathrm{~min}$ (see fig. 2).

The shaft of the upper part of the fluxer extends out a hole in the door of the muffle furnace and is attached to a motor that rocks the crucibles back and forth. During this time, the tilting action of the upper portion of the fluxer homogenizes the molten mixture. After the mixing phase of the fusion is completed, the crucible support rack is inverted, pouring the fused mixture into the molds. The fluxer then is removed from the muffle furnace and cooled to near room temperature.

An important feature of this method is a two-piece mold designed to create discs that have smooth, flat surfaces (Taggart and Wahlberg, 1980b). The mold consists of a Pt-Au ring and disc, shown in figure 3, which are wired together with platinum-rhodium wire during use, but which can be taken apart so that the surface of the platinum disc can be ground flat and polished smooth.

Commercial molds used for casting fusion discs are made from Pt-Au sheets ranging from about 0.5 to $1 \mathrm{~mm}$ thick. During use, the molds can become deformed, producing discs whose analytical surfaces are concave or convex. Periodic flattening and refinishing of the mold is difficult and only partially successful. Resurfacing of each glass disc before its use has been shown to be highly successful for improving analytical precision and may be performed again at a later time to remove any radiationdamaged material or surface contamination. If the initial flattening is done manually, however, then the method is very time consuming and tedious.

The two-piece mold results in a significant savings in manpower, compared to grinding each sample disc, and virtually eliminates contamination of the analytical surface during grinding of the glass discs. Experience indicates that the molds need to be refinished after about 400 samples have been run. The fluxer and molds were designed in the Denver laboratory (Taggart and Wahlberg, 1980a, b) where over 40,000 samples were prepared in the first $6 \mathrm{yr}$ of use. This sample-preparation technique is now used in the three centers for majorelement determinations.

Tests have shown that some of the important factors in creating a homogeneous disc are to have the particle size of the sample and flux be similar, to ensure a good mechanical mixture of the flux and sample before fusing, and to use flux with a moisture content less than 0.4 percent.

Often, insufficient sample is available to prepare a standard fusion disc. When major and minor rockforming elements are to be analyzed on a small sample in the Menlo Park laboratory, the following alternative preparation procedure is used. A 0.125 -g portion of sample is mixed with $0.725-\mathrm{g}$ of lithium tetraborate. The mixture is transferred completely to a graphite crucible and fused in a muffle furnace at $1,100^{\circ} \mathrm{C}$ for $15 \mathrm{~min}$. The crucible then is removed from the furnace and allowed to cool in a desiccator. The glass bead is removed from the crucible and weighed. Enough chromatographic cellulose powder (Whatman CF11) is added to give a combined weight of $1.000 \mathrm{~g}$. The bead then is crushed and transferred completely along with the cellulose to an acrylic mixing vial with tungsten carbide end caps and a tungsten carbide ball. The mixture is shaken in a mixer mill for $10 \mathrm{~min}$. Using a special die (Fabbi, 1970), the final mixture then is pressed hydraulically with a backing of 72-percent cellulose acetate and 28-percent wax at 25,000 psi for $1 \mathrm{~min}$. The pelletized sample is removed from the die and stored in a desiccator until time for analysis.

\section{Samples That Present Difficulties in Preparation}

Samples that contain metals that alloy readily with platinum are a problem when using the fusion technique. Also, metals in their reduced form alloy much more readily with platinum than those in higher oxidation states. One of the added features of the in-muffle furnace fluxer is that the environment of the furnace is oxidizing rather than being heated by a propane or natural gas flame. In the muffle furnace, the sample can routinely contain up to 25 weight-percent pyrite. Even 5 weightpercent chalcopyrite and 10 weight-percent volkonskoite (a chromium clay mineral) have been run, especially if information about their presence is given so that sample weights can be adjusted. Lead, arsenic, and tellurium minerals, however, are totally unacceptable over 0.2 weight-percent, even in oxidized minerals such as cerussite or olivenite. Other elements that can cause problems probably exist but have not yet been encountered at sufficiently abundant levels in submitted samples.

Elements that cause sticking to the platinumware also are a problem. Of the elements that can cause 

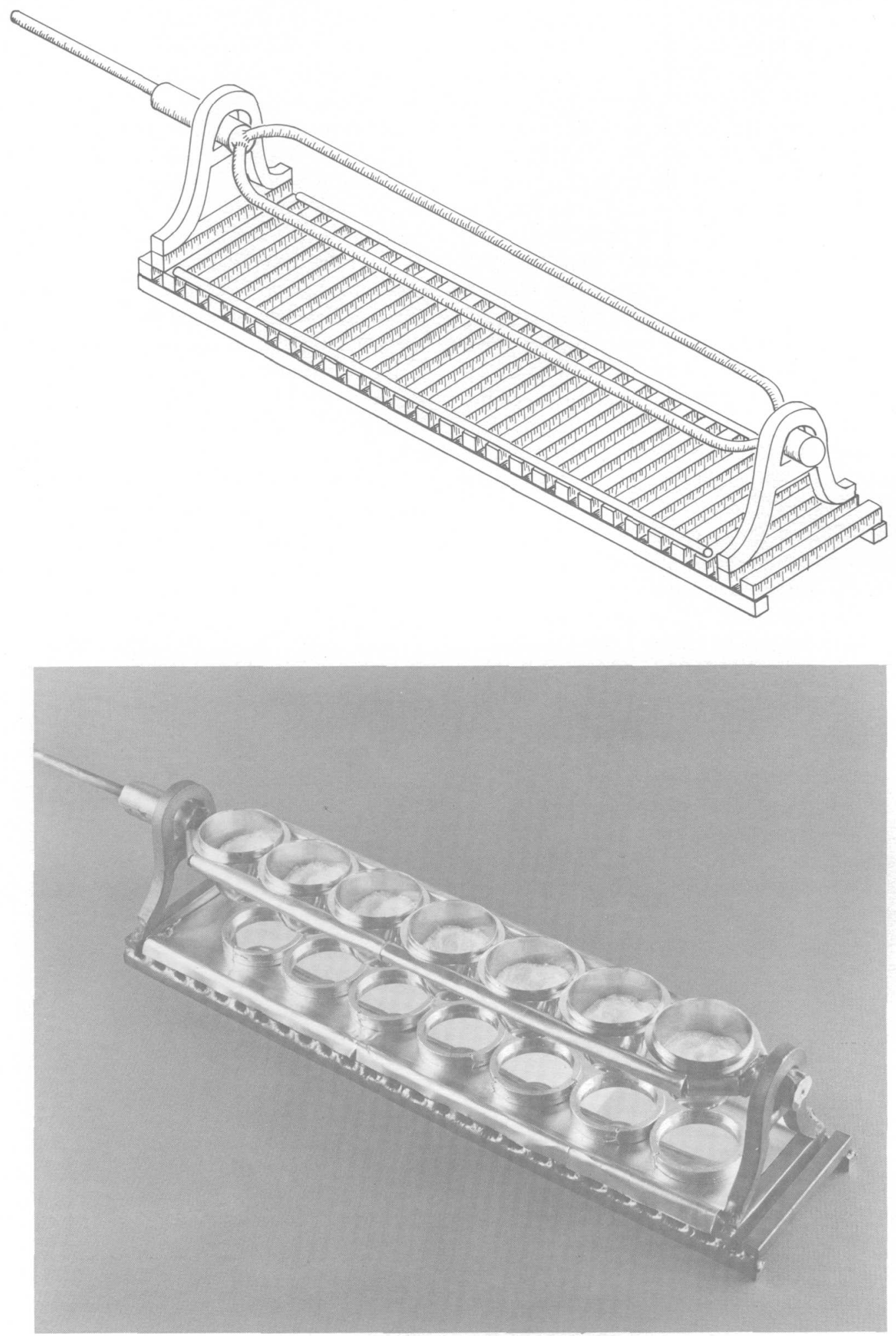

Figure 2. Oblique drawing of the $\mathrm{Ni}-\mathrm{Cr}$ body of the in-furnace device (upper). Oblique photograph of fluxer clad with platinum sheet and loaded with crucibles and molds before loading in a muffle furnace (lower) (Taggart and Wahlberg, 1980a). 

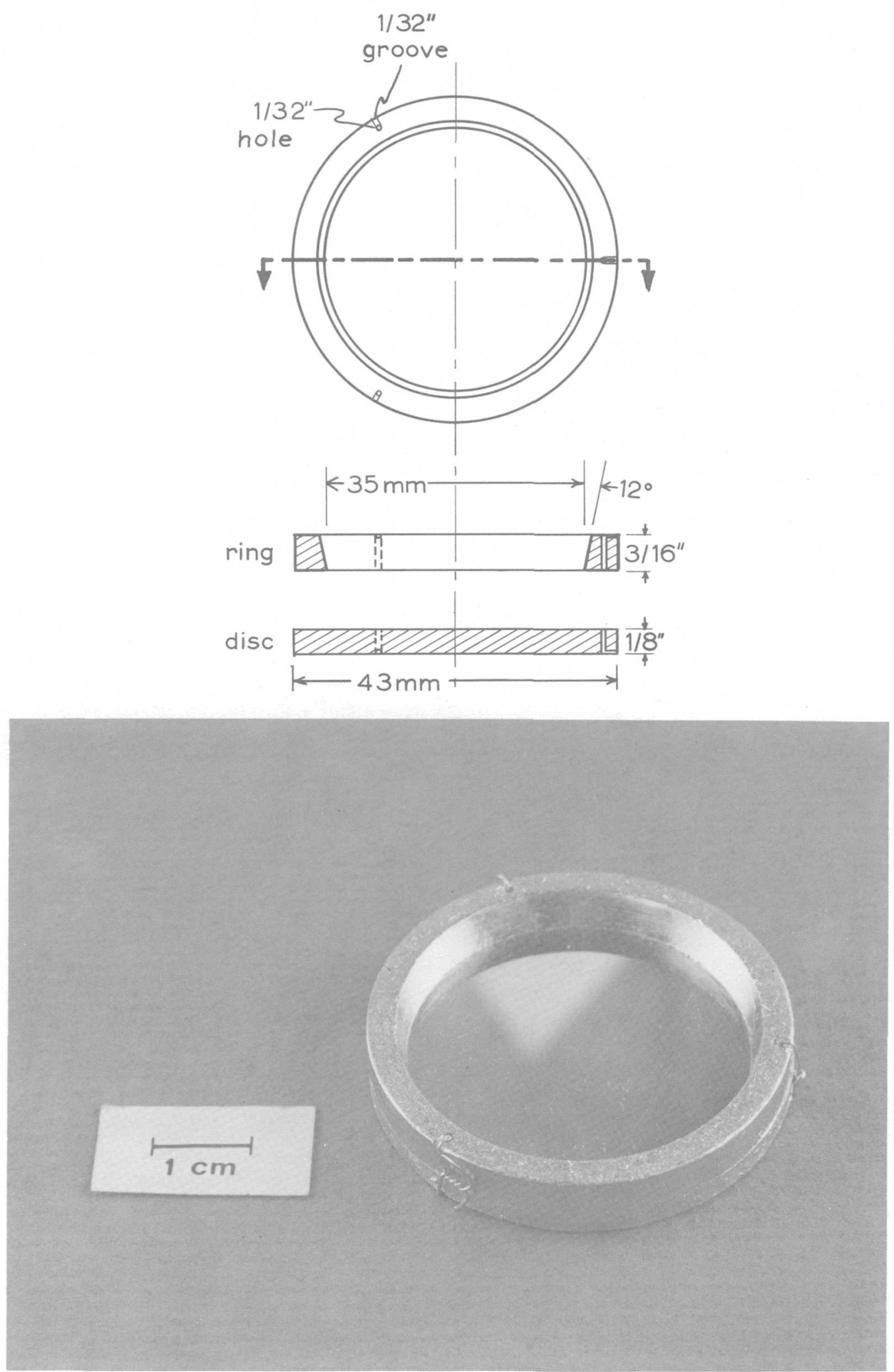

Figure 3. Measured drawing of a two-piece 95-percent Pt:5-percent Au mold for casting fused discs for major-element analysis (upper). Oblique photograph of a ring and disc wired together with 82-percent $\mathrm{Pt}$ :18-percent $\mathrm{Rh}$ wire before use. $\mathrm{Rh}$ alloy wire is used because it is stronger than $\mathrm{Pt}$ wire, and the $1 / 32$-in. groove prevents the wire from being crushed and weakened during use (lower) (Taggart and Wahlberg, 1980b). 
sticking, only chromium, nickel, and copper are encountered at high enough abundance levels to cause problems; by far the worst of these is copper. The problem can be diminished by adding more of the nonwetting agent on the second try, using only a half-weight sample, or both. Samples with more than 10 percent copper should not be analyzed by XRF except by nonfusion sample-preparation techniques.

Iron- or manganese-rich samples have a double problem. To give good results at low levels, the $\mathrm{X}$-ray spectrometer is set up to achieve maximum countrate for all elements, including iron and manganese. Because iron and manganese have fairly energetic $X$-rays and high fluorescence yields and because some geologic environments have very high concentrations of either element, it is possible to have so many characteristic $\mathrm{X}$-ray photons that the detectors fail to record all the events and experience dead time. On the Philips $\mathbb{P W} 1600$, for example, unacceptably high dead times occur at $\mathrm{MnO}$ concentrations of greater than 32 percent and at $\mathrm{Fe}_{2} \mathrm{O}_{3}$ concentrations of greater than 28 percent. Furthermore, especially with $\mathbb{F e}$, the more of the element in the glass disc, the greater the likelihood that the disc will devitrify during cooling and need to be prepared again. Both of these problems can be diminished by preparing the disc with a partial weight sample.

\section{COab-ASh Samples}

For sample-preparation considerations, organicrich materials fall into two categories - those having 5-25 percent organic matter and those having $>25$ percent organic matter. Samples that contain between 5 and 25 percent organic matter, such as oil shale, black shales, soils, and so forth, occasionally support combustion during ignition but, more frequently, experience small explosions that expel sample from the crucible. If very little sample is present, then potential problems with the ignition process need to be anticipated before analysis. Those samples with less than 5 percent organic matter are not a problem during fluxing when the samples are preignited before fusion. Those that have $>25$ percent organic matter, such as coal, peat, vegetation, and so forth, support combustion and give problems during the ignition stage of the sample preparation. Furthermore, because the organic matter serves to "dilute" the elements of interest in the sample, it serves to reduce the count rates and, therefore, to reduce the precision of an XRF analysis. Thus, for samples with $>25$ percent organic matter, the sample is ashed slowly, and then the analysis is performed on the full sample weight of the ash. Currently, all coal-ash samples are analyzed in the Reston laboratory on the Diano XRD8300.

A sample of ashed coal weighing $0.800 \mathrm{~g}$ is placed in a $\mathbb{P t}$-Au crucible along with $5.20 \mathrm{~g}$ of lithium tetraborate and $0.05 \mathrm{~mL}$ of 15 percent $\mathrm{HBr}$ solution, which acts as a nonwetting agent. The mixture is fused at $1,100^{\circ} \mathrm{C}$ by using the Claisse fluxer (the Denver fluxer could also be used for the fusion). Then, the melt is poured into the mold to form the disc. The discs are stored in a desiccator until analyzed.

\section{Whole COal}

Whole coal is analyzed directly for $\mathbb{P}_{2} \mathrm{O}_{5}$ and chlorine in the Reston laboratory. Coal samples are ground to -200 mesh and prepared for WDXRF analysis as a pressed briquette. An aliquot of whole coal is dried in a porcelain crucible in an oven at $105^{\circ} \mathrm{C}$ overnight and stored in a desiccator. Then, $0.500 \mathrm{~g}$ of the dried coal and $0.500 \mathrm{~g}$ of cellulose powder (Whatman microgranular CC31 cellulose powder) are weighed on an analytical balance. The whole coal and cellulose are transferred to a small plastic vial along with two small plastic beads. The vial containing the coal-cellulose mixture then is placed on a mixer mill for $10 \mathrm{~min}$. The mixture is stored in a desiccator until it is ready to be pressed into a pellet. A 1-in.-diameter pellet is then formed with a hydraulic press by using Whatman fibrous $\mathrm{CF} 1$ cellulose powder as the backing material for the coal-cellulose mixture. The pellet is pressed at 30,000 psi for $30 \mathrm{~s}$ and stored in a desiccator until analyzed.

\section{Trace-El_ment Analysis}

Sample preparation for trace-element analysis requires minimum dilution because the $X$-ray count rate is low. Most trace-element analyses using XRF spectrometry are performed on the EDXRF spectrometer. However, a few elements cannot be determined by EDXRF because of $X$-ray line interferences or inadequate detector resolution. These elements can be determined by using WDXRF because of the greater resolution of the spectrometer or because a different X-ray source is used for excitation. Two elements that have been determined by WDXRF are vanadium and cobalt. Before the acquisition of the EDXRF spectrometers, rubidium, strontium, and barium also were determined by using WDXRF. The sample-preparation methods described below have been used in the Reston and Menlo Park laboratories for more than $15 \mathrm{yr}$.

A 0.850 -g portion of rock sample is mixed with $0.150 \mathrm{~g}$ of chromatographic cellulose powder (Whatman $\mathrm{CF} 11$ ) and transferred quantitatively to an acrylic mixing vial with tungsten carbide end caps and a tungsten carbide ball. The mixture is shaken in a mixer mill for $10 \mathrm{~min}$. By using the special die described by Fabbi (1970), the mixture then is pressed into a briquette with a backing of 
72 percent cellulose acetate and 28 percent wax at 25,000 psi for $1 \mathrm{~min}$. The sample briquette is removed from the die and stored in a desiccator until it is time to be analyzed. Samples and standards are prepared following the same procedure and the calibration for trace elements performed on the Diano XRD8600 X-ray spectrometer. As an example, typical calibration data for vanadium is given below. The coefficient of determination is 0.9991 . The standard error of estimate at the 68-percent confidence level is $4 \mathrm{ppm}$ over the calibration range of $2-600$ $\mathrm{ppm}$. The vanadium intensity is corrected for three effecting elements (titanium, calcium, and iron) by using equation 12.

An alternative sample preparation used in the Reston laboratory is identical to the whole coal method described above. The interelement matrix effects, mostly absorption of analyte $\mathrm{K}_{\alpha} \mathrm{X}$-ray line by the matrix elements, are corrected for by measuring the background $\mathrm{X}$-ray intensity on each side of the $\mathrm{K}_{\alpha} \mathrm{X}$-ray line and by calculating the peak $\mathrm{X}$-ray intensity to the background intensity ratio. This ratio is calculated for each standard and the data plotted versus the known concentration value. Rubidium, strontium, and barium have been analyzed by this method with a lower limit of detection of 2 $\mathrm{ppm}$ and a relative standard deviation of \pm 5 percent at the 200-ppm level.

\section{PRECISION AND ACCURACY}

Precision is the deviation of a set of determinations from their mean (random error), and accuracy is the degree of conformity with a standard (elimination of systematic plus random error) (Bates and Jackson, 1980). A technique that can give repeatedly the same data on a sample, therefore, is precise. A highly precise technique may yield biased results because of sample preparation and calibration errors and, therefore, be inaccurate. To accomplish high-quality analyses, it is necessary to have an unbiased, precise method.

\section{Short-Term Drift and Stability}

In the XRF method, the two principal sources of short-term imprecision are the instability of the instrument and the imprecision of the sample preparation. Table 2 summarizes some of the tests performed on a Philips PW1600 X-ray spectrometer over the past 7 yr. Because of the large number of repetitions required by these tests, it was neither feasible nor desirable to acquire and use (and, therefore, help to deplete) a valuable international standard reference material. Instead, approximately $25 \mathrm{lb}$ of basalt from Table Mountain, Golden, Colorado, was ground to -100 mesh and homog- enized to be used for an in-house standard, which is not available for distribution outside the Geological Survey. The standard was named Baker's basalt (BB-1) in 1978 for the person who collected and prepared it. (BB-1 is also the designation given to a basalt standard from the Federal University of Bahia, Brazil. No relation exists between the two standards.) The composition of the Table Mountain basalt is given in the first column of table 2 . From this composition and the count rate on the respective channnels, it is possible to calculate the theoretical errors introduced by counting statistics using the formula

$$
\sigma=\sqrt{\mathrm{N}},
$$

where $\sigma$ is the standard deviation due to random counting error and $\mathbf{N}$ is the total number of events counted. Column 2 of table 2 gives the theoretical contribution to error, in weight-percent of the appropriate oxide, from counting statistics for a disc of $\mathrm{BB}-1$ on the Phillips PW1600 under normal operating conditions. This represents the best precision that can be expected from any $\mathrm{X}$-ray spectrometer (without increasing the total number of counts by changing the instrument parameters) and, commonly, has been incorrectly given as the precision of an instrument or a technique. To measure the PW1600s precision, one disc of BB-1 was counted 50 times in $1.5 \mathrm{~h}$. Column 3 of table 2 gives the standard deviations for the data which show remarkably good agreement with $\sigma$ based only on counting statistics.

As a test of the Denver fluxer-fusion samplepreparation procedure (Taggart and Wahlberg, 1980a, b; Taggart and others, 1981), 49 lithium tetraborate fusion discs of BB-1 were prepared and counted once each over a period of $1.5 \mathrm{hr}$; the results are given in column 4 of table 2. The estimated contribution to error from sample preparation is about 0.2 percent of the elemental concentration. This agrees very well with data presented in Taggart and Wahlberg (1980b); the starting mixture of sample and flux was fused and mixed while molten in a $360-\mathrm{g}$ batch and then cooled, pulverized, and remelted into glass discs, thus virtually eliminating the question of homogeneity. This deviation in composition corresponds to the same deviation observed when the sample is positioned only 0.0005 in. further away from the X-ray tube and most likely is the limit of the preparation of the surface of the mold.

\section{Long-Term Drift}

The detected intensity of emitted X-rays will change over a period of time due to changes in room temperature, barometric pressure, voltage changes of power supplies or house power, and many other related factors. To 
correct for these "long-term" effects, a standard or sample is selected to be the drift monitor. The monitor must contain sufficient concentration of each element to ensure statistically acceptable count rates. During the original calibration, the count rate on the monitor is determined. In subsequent analyses, the monitor also is analyzed and then ratioed to its intensity values obtained during the original calibration. The drift monitor corrects for changes in X-ray intensity due to slowly changing, long-term effects, but it cannot correct for short-term effects, such as transient "spikes" in the detector system or voltage fluctuations of short time duration. Also, it will not adequately handle significant changes in operating parameters caused by such events as installing a new X-ray tube or a power supply.

Column 5 of table 2 shows data gathered from one standard disc of BB-1 determined on the Philips PW1600 and gives the standard deviation for one disc of $\mathrm{BB}-1$ counted 62 times in a 6-mo period from July to December 1981. Column 6 shows the standard deviation for another separately prepared disc of $\mathrm{BB}-1$ counted 62 times in a 5-mo period from January to May 1985, showing that precision of the instrument has remained the same or improved slightly on various channels over $3.5 \mathrm{yr}$. Table 3 shows an example of the accuracy determined on the Philips PW1600 with the analyses of seven standards in 1980 (Taggart and others, 1981) and again in 1985 (each analysis run separately on a separately prepared sample), which illustrates the method's ability to correct for longterm drift.

\section{Selection of Calbration Standards}

To calibrate an instrument, the analyst must use materials of known composition to determine a mathematical relation between the $X$-ray count rate of the analyte and its abundance. Rather than tie an instrument's calibration to a manufacturer's certification of a chemical standard, it is preferred to select the "best value" for the then-available suitable standard reference materials (SRM's). All X-ray laboratories at the Survey use Abbey's (1980) compilation of "best values" for their highprecision major-element calibration, selecting only those silicate standards that are relatively well established and dependable, which includes 42 worldwide SRM's.

The standards used in calibrating the Diano XRD8300 for the analysis of ashed coals are silicate reference standards available from the U.S. Geological Survey, the National Bureau of Standards, the University of Nancy in France, and rocks analyzed from an in-house round robin. In all, a total of 32 standards are used in the calibration for the $\mathbf{1 0}$ major and minor oxides plus sulfur, with the result that the following range of values (in percent) is covered for each oxide:

$$
\begin{array}{llll}
\mathrm{Na}_{2} \mathrm{O} & 0.06-8.37 & \mathrm{~K}_{2} \mathrm{O} & 0.03-10.03 \\
\mathrm{MgO} & 0.10-43.50 & \mathrm{CaO} & 0.08-50.50 \\
\mathrm{Al}_{2} \mathrm{O}_{3} & 0.73-54.53 & \mathrm{TiO}_{2} & 0.01-2.69 \\
\mathrm{SiO}_{2} & 4.43-87.21 & \mathrm{MnO} & 0.01-0.77 \\
\mathrm{P}_{2} \mathrm{O}_{5} & 0.01-34.50 & \mathrm{Fe}_{2} \mathrm{O}_{3} & 1.00-25.76
\end{array}
$$

Standards for the analysis of sulfur are prepared synthetically. Sulfur in the form of calcium sulfate is added to an appropriate rock standard such as basalt BIR-1, and serial dilutions are made to cover the range of 0.1-10.0 percent. Sulfur is lost during the ashing of the whole coal, so two discs of each standard are prepared. The discs are counted, and then one of the discs is pulverized and independently analyzed for the remaining sulfur content by a Leco sulfur analyzer. The Leco values then are used in the calibration of the X-ray spectrometer, and the sulfur content of the unpulverized discs may be determined, allowing these discs to be used for instrument recalibration at a future time.

When prepared as fused discs, the ratio of the reference standard material to the lithium tetraborate flux is 1 to 6.5 . The dilution of the standard material minimizes interelement effects. This allows the use of the simple linear regression equation model to be used in preparing the calibration for all the oxides.

Eighteen reference standards are used in the calibration of the Diano XRD8300 for phosphorus in whole coals, ranging from 0.0088 to 0.91 percent $\mathbb{P}_{2} \mathrm{O}_{5}$. Chlorine calibrations are performed with nine standards covering the range of $0.008-0.234$ percent $C$. These standards come from a variety of sources. An American Society for Testing and Materials round robin produced unpublished $\mathrm{P}_{2} \mathrm{O}_{5}$ values for five standards, and National Bureau of Standards coal standards 1632 and 1632a have been analyzed for $\mathbb{P}_{2} \mathrm{O}_{5}$ and $\mathrm{Cl}$ by Ondov and others (1975) and Germani and others (1980). A coal sample from the Illinois State Geological Survey was analyzed in a round robin for $\mathbb{P}_{2} \mathrm{O}_{5}$, and a group of coals from three U.S. powerplants were analyzed by instrumental neutron activation analysis for $\mathrm{Cl}$. Neither of these amalyses have been published. Additional in-house standards for $\mathbb{P}_{2} \mathrm{O}_{5}$ were created by taking coal samples that represented a wide range of concentrations. These coals were ashed and analyzed in triplicate by inductively coupled plasmaatomic emission spectrometry and $X \mathbb{R} F$, and the whole coal values were calculated. 
Table 3. Analyses in 1981 and 1985 of seven igneous rock standards (in weight percent) by the high-precision X-ray fluorescence majors method

[XRF (X-ray fluorescence) (1981) from Taggart and others (1981), Abbey from Abbey (1978), XRF (1985) current XRF values performed in July 1985]

\begin{tabular}{|c|c|c|c|c|c|c|c|c|c|c|}
\hline & $\mathrm{SiO}_{2}$ & $\mathrm{Al}_{2} \mathrm{O}_{3}$ & $\mathrm{IFe}_{2} \mathrm{O}_{3}$ & $\mathrm{MgO}$ & $\mathrm{CaO}$ & $\mathrm{Na}_{2} \mathrm{O}$ & $\mathrm{K}_{2} \mathrm{O}$ & $\mathrm{THO}_{2}$ & $P_{2} O_{5}$ & MnO \\
\hline \multicolumn{11}{|l|}{ Andes ite, AGV-1: } \\
\hline XRF (1981) & 59.9 & 17.2 & 6.88 & 1.57 & 5.11 & 4.25 & 2.99 & 1.08 & 0.52 & 0.08 \\
\hline Abbey & 59.72 & 17.22 & 6.84 & 1.55 & 5.00 & 4.31 & 2.93 & 1.05 & 0.50 & 0.10 \\
\hline XRF (1985) & 59.4 & 17.1 & 6.80 & 1.55 & 5.03 & 4.19 & 2.91 & 1.07 & 0.51 & 0.09 \\
\hline \multicolumn{11}{|l|}{ Dunite, DTS-1: } \\
\hline XRF (1981) & 40.6 & 0.18 & 8.84 & 49.8 & 0.11 & $<0.2$ & $<0.05$ & $<0.02$ & $<0.05$ & 0.11 \\
\hline Abbey & 40.68 & 0.29 & 8.60 & 49.83 & 0.13 & 0.01 & 0.00 & 0.01 & 0.00 & 0.11 \\
\hline XRF (1985) & 40.7 & 0.26 & 8.90 & 50.2 & 0.13 & $<0.15$ & $<0.02$ & $<0.02$ & $<0.05$ & 0.13 \\
\hline \multicolumn{11}{|l|}{ Granite, G-2: } \\
\hline XPF (1981) & 69.9 & 15.6 & 2.73 & 0.81 & 1.98 & 3.94 & 4.54 & 0.49 & 0.13 & 0.02 \\
\hline Abbey & 69.19 & 15.35 & 2.67 & 0.71 & 1.98 & 4.06 & 4.52 & 0.50 & 0.14 & 0.04 \\
\hline XRF (1985) & 69.0 & 25.2 & 2.64 & 0.85 & 1.95 & 3.91 & 4.45 & 0.49 & 0.13 & 0.02 \\
\hline \multicolumn{11}{|c|}{ Granodiorite, GSP-1: } \\
\hline XPF (1981) & 67.2 & 15.2 & 4.32 & 1.01 & 2.04 & 2.61 & 5.51 & 0.66 & 0.29 & 0.02 \\
\hline Abbey & 67.31 & 15.19 & 4.33 & 0.96 & 2.02 & 2.80 & 5.53 & 0.66 & 0.28 & 0.04 \\
\hline XRF (1985) & 67.0 & 14.9 & 4.32 & 0.96 & 2.02 & 2.67 & 5.47 & 0.68 & 0.29 & 0.03 \\
\hline \multicolumn{11}{|l|}{ Basalt, BRM: } \\
\hline XPF (1981) & 38.4 & 10.2 & 13.0 & 13.3 & 14.0 & 2.91 & 1.38 & 2.70 & 1.10 & 0.18 \\
\hline Abbey & 38.39 & 10.25 & 12.9 & 13.35 & 13.87 & 3.07 & 1.41 & 2.61 & 1.05 & 0.20 \\
\hline XRF (1985) & 38.6 & 9.89 & 13.1 & 13.3 & 14.0 & 3.01 & 1.36 & 2.71 & 1.09 & 0.20 \\
\hline \multicolumn{11}{|l|}{ Granite, GSN: } \\
\hline XRF (1981) & 66.4 & 15.0 & 3.73 & 2.28 & 2.55 & 3.61 & 4.69 & 0.67 & 0.28 & 0.04 \\
\hline Abbey & 65.98 & 14.71 & 3.75 & 2.31 & 2.51 & 3.78 & 4.64 & 0.68 & 0.28 & 0.06 \\
\hline XRF (1985) & 65.7 & 14.5 & 3.69 & 2.22 & 2.51 & 3.67 & 4.60 & 0.66 & 0.28 & 0.05 \\
\hline \multicolumn{9}{|l|}{ SAPU-3-L: } & & \\
\hline XPF (1981) & 52.1 & 13.6 & 9.97 & 0.32 & 3.18 & 8.23 & 5.38 & 0.48 & 0.05 & 0.74 \\
\hline Abbey & 52.45 & 13.59 & 9.96 & 0.28 & 3.24 & 8.30 & 5.46 & 0.49 & 0.06 & 0.76 \\
\hline XRF (1985) & 52.0 & 13.3 & 9.93 & 0.29 & 3.15 & 8.25 & 5.31 & 0.49 & 0.05 & 0.79 \\
\hline
\end{tabular}




\section{REFERENCES CITED}

Abbey, Sydney, 1978, U.S.G.S. II revisited: Geostandards Newsletter, 2, p. 141-146.

1980, Studies in "standard samples" for use in the general analysis of silicate rocks and minerals, part 6; 1979 edition of "usable" values: Geological Survey of Canada paper 80-14, $30 \mathrm{p}$.

Adler, Isidore, 1966, X-ray emission spectrography in geology: New York, Elsevier Publishing Co., 258 p.

Alley, B. J., and Myers, R. H., 1965, Corrections for matrix effects in X-ray fluorescence analysis using multiple regression methods: Analytical Chemistry, v. 37, p. 1685-1690.

Baker, J. W., 1982, Volatilization of sulfur in fusion techniques for preparation of discs for X-ray fluorescence analysis: Advances in X-Ray Analysis, v. 25, p. 91-94.

Bates, R. L., and Jackson, J. A., editors, 1980, Glossary of geology: Falls Church, Va., American Geological Institute, $749 \mathrm{p}$.

Bertin, E. P., 1975, Principles and practices of X-ray spectrometric analysis: New York, Plenum Press, 1079 p.

Claisse, F., 1957, Accurate X-ray fluorescence analysis without standards: Norelco Reporter, v. 4, p. 3-7.

Claisse, F., and Quintin, M., 1967, Generalization of the Lachance-Traill method for the correction of the matrix effect in X-ray fluorescence analysis: Canadian Spectroscopy, v. 12, p. 129.

Cuttitta, F., and Rose, H. J., Jr., 1968, Slope-ratio technique for the determination of trace elements by X-ray spectroscopy-A new approach to matrix problems: Applied Spectroscopy, v. 22, p. 321.

deJongh, W. K., 1973, X-ray fluorescence analysis applying theoretical matrix corrections-Stainless steel: X-ray Spectrometry, v. 2, p. 151-158.

Fabbi, B. P., 1970, A die for pelletizing samples for X-ray fluorescence analysis, in Geological Survey Research 1970: U.S. Geological Survey Professional Paper 700-B, p. B187-B189.

1972, A refined fusion X-ray fluorescence technique and determination of major and minor elements in silicate standards: American Mineralogist, v. 57, p. 237-245.

1978, Geology, [Chap] 12, in Herglotz, H. K., and Birks, L. S., eds., X-ray spectrometry: New York, Marcel Dekker, Inc. (Practical spectroscopy series, v. 2), $518 \mathrm{p}$.

Germani, M. S., Gokmen, Inci, Sigleo, A. C., Kowalczyk, G. S., Olmez, Ilhan, Small, A. M., Anderson, D. L., Failey, M. P., Gulovali, M. C., Choquette, C. E., Lepel, E. A., Gordon, G. E., 1980, and Zoller, W. H., Elemental concentrations in the National Bureau of Standards' bituminous and subbituminous coal standard reference materials: Analytical Chemistry, 11, 2, p. 240-245.

Johann, H. H., 1931, Intense X-ray spectra obtained with curved crystals: Zeitschrift fur Physik, v. 69, p. 185-206.

Johansson, T., 1933, New focusing X-ray spectrometer: Zeitschrift fur Physik, v. 82, p. 507-528.

Lachance, G. R., 1983, Introduction to alpha coefficients: Sainte-Foy, Quebec, Corporation Scientifique Claisse, Inc., 189 p.
Lachance, G. R. and Traill, R. J., 1966, A practical solution to the matrix problem in X-ray analysis, I. Method: Canadian Spectroscopy, v. 11, p. 43-48.

Lindsay, J. R., Larson, R. R., Rose, H. J., Jr, and Werre, R. W., A fully-focussing $\mathrm{X}$-ray spectrometer with application to geochemical analysis: American Chemical Society, 176th Annual Meeting, Miami Beach, Fla., 1978, Abstracts, paper no. 17.

Lindsay, J. R., Rose, H. J., Jr., and Larson, R. R., 1982, An evaluation of the Rasberry-Heinrich model for the analysis of silicate rocks: Applied Spectroscopy, v. 36, p. 520-523.

Lucas-Tooth, H. J., and Pyne, C., 1964, The accurate determination of major constituents by $X$-ray fluorescent analysis in the presence of large interelement effects: Advances in X-ray Analysis, v. 7, p. 523-541.

Mitchell, B. J., and Hopper, F. N., 1966, Digital computer calculation and correction of matrix effects in X-ray spectroscopy: Applied Spectroscopy, v. 20, p. 172-180.

Moseley, H. G. J., 1913, High-frequency spectra of the elements: Philosophical Magazine, v. 26, p. 1024-1034.

Ondov, J. M., Zoller, W. H., Olmex, Ilhan, Aras, N. K., Gordon, G. E., Rancitelli, L. A., Abel, K. H., Filby, R. H., Shah, K. R., and Ragaini, R. C., 1975, Elemental concentrations in the National Bureau of Standards' environmental coal and fly ash standard reference materials: Analytical Chemistry, 47, 7, p. 1102-1109.

Price, B. J., Prissette, J. P., and Juchli, K., 1985, A review of long and ultra long wavelength XRF analysis: International Labmanual Chromatography/Spectroscopy.

Rasberry, S.D., and Heinrich, K.F.J., 1974, Calibration for interelement effects in X-ray fluorescence analysis: Analytical Chemistry, v. 46, p. 81-89.

Rose, H.J., Jr., Adler, Isidore, and Flanagan, F.J., 1962, Use of $\mathrm{La}_{2} \mathrm{O}_{9}$ as a heavy absorber in the X-ray fluorescence analysis of silicate rocks: U.S. Geological Survey, Professional paper 450-B, p. B80-B82.

1963, X-ray fluorescence analysis of the light elements in rocks and minerals: Applied Spectroscopy, v. 17, p. 81-85.

Rose, H.J., Jr., and Cuttitta, Frank, 1968a, X-ray fluorescence spectroscopy in the analysis of ores, minerals, and waters: Advances in X-Ray Analysis, v.11, p.23.

-1968b, X-ray fluorescence analysis of individual rare earths in complex minerals: Applied Spectroscopy, v. 22, p. 426-430.

Spinella, R.A., and Lindsay, J.R., 1981, Universal X-ray instrument automation and data reduction: 30th Annual Conference on Applications of X-Ray Analysis, Denver, Colorado, 1981, Abstracts, p. 42.

Taggart, J.E., Jr., Lichte, F.E., and Wahlberg, J.S., 1981, Methods of analysis of sample using X-ray fluorescence and induction coupled plasma spectroscopy, in Lipman,P.W., and Mullineaux, D.R., The 1980 eruption of Mount St. Helens, Washington: U.S. Geological Survey Professional Paper 1250, p. 683-687.

Taggart, J.E., Jr., and Wahlberg, J.S., 1980a, A new in-muffle automatic fluxer design for casting glass discs for $\mathrm{X}$-ray fluorescence analysis: Federation of Analytical Chemistry and Spectroscopy Societies, 7th Annual Meeting, Philadelphia, Pennsylvania, 1980, Abstracts, paper no. 327a. 
1980b, New mold design for casting fused samples: Advances in X-Ray Analysis, v. 23, p. 257-261.
Tertian, R., and Claisse, F., 1982, Principles of quantitative Xray fluorescence analysis: New York, Wiley-Heyden, $385 \mathrm{p}$. 


\section{CHAPTER F}

\section{Energy-Dispersive X-Ray Fluorescence Spectrometry}

By R. G. JOHNSON and B.-S. L. KING

U.S. GEOLOGICAL SURVEY BULLETIN 1770

Methods for Geochemical Analysis 



\section{CONTENTS}

Abstract F1

Introduction $\mathbf{F 1}$

Experimental F2

Sample preparation $\mathbf{F 2}$

Quantitative analysis F2

Standard reference materials F3

Instrumentation $\mathbf{F 4}$

Sensitivity, precision, and accuracy $\mathbf{F 4}$

References cited F5

FIGURE

1. Typical energy-dispersive $\mathrm{X}$-ray fluorescence spectrum with X-ray energy plotted with log of intensity F2

TABLE

1. Lower limit of detection and upper limit of standard curve for typical energy-dispersive X-ray fluorescence determinations F4 



\title{
Energy-Dispersive X-Ray Fluorescence Spectrometry
}

\author{
By R. G. Johnson and B.-S. L. King
}

\section{Abstract}

The application of energy-dispersive X-ray fluorescence analysis to geological samples is described. Energydispersive $X$-ray fluorescence can be applied to the determination of those elements having an atomic number greater than 10 (neon). The technique can be applied to majorelement analyses but with less precision than wavelengthdispersive $X$-ray fluorescence and with much lower sensitivity for sodium and magnesium. The use of secondary targets for excitation makes energy-dispersive $X$-ray fluorescence a useful tool for several groups of trace elements. Current applications are directed primarily to the determination of 12 trace elements in silicate rocks.

\section{INTRODUCTION}

Energy-dispersive X-ray fluorescence (EDXRF) spectrometry is a technique for qualitative or quantitative elemental analysis of solid or liquid materials. Although it is very similar to wavelength-dispersive $\mathrm{X}$-ray fluorescence (WDXRF) spectrometry in principle, some important differences do exist. Typical EDXRF spectrometers can collect and display information about every element (from sodium to uranium) in a sample simultaneously and in real time, making the technique extremely versatile for identifying elemental constituents in a sample very rapidly. Unfortunately, EDXRF suffers from more severe line overlaps than does WDXRF because a different detector system is used. Also, the sensitivity for the light elements (sodium and magnesium) is much less than with WDXRF, and, consequently, the lower limit of detection is worse (0.02 percent sodium for WDXRF vs 2 percent sodium for EDXRF). Other minor differences have been noted, but many of the same sample preparation requirements and mathematical correction schemes are identical for the two methods.

$\mathrm{X}$-ray fluorescence analysis entails the excitation of $\mathrm{X}$-rays within a sample and their subsequent detection and measurement. Sample excitation normally is accomplished with an $\mathrm{X}$-ray tube, although radioactive sources, such as ${ }^{55} \mathrm{Fe}$ and ${ }^{109} \mathrm{Cd}$, also can be used. The X-ray tube produces a polychromatic beam of $\mathrm{X}$-radiation and the characteristic radiation of its anode. This radiation is used to irradiate either the sample directly or a "secondary target," which, in turn, irradiates the sample with monoenergetic X-rays. Polychromatic excitation has an advantage in its capacity to excite a wide range of elements; however, the resulting spectrum is superimposed over a high background. Monochromatic X-rays excite a more limited range of elements, but the backgrounds are very low. Therefore, secondary-target excitation often is used for trace-element analysis where the lower background is important, and direct excitation is used in major-element analysis where excitation of a wide range of elements is required.

During sample irradiation, inner shell electrons of the elements in the sample absorb specific X-ray photons and are ejected from the atom. Rearrangement of the remaining electrons to fill these vacancies causes the emission of so-called fluorescent $\mathrm{X}$-rays, whose energies are characteristic of the elements from which they originate.

Lithium drifted silicon detectors ( $\mathrm{Si}(\mathrm{Li}))$ typically are used in EDXRF analysis. X-rays emitted by the sample are absorbed in the detector, which acts as a diode in converting these incident $\mathrm{X}$-rays to electronic pulses whose amplitudes are proportional to the energies of the corresponding $\mathrm{X}$-rays. Pulses then are processed and sorted according to amplitude; the entire range of pulse amplitudes is divided into 1,024 intervals, and those pulses falling within each interval are counted in a multichannel analyzer. An EDXRF spectrum is actually a histogram of the number of counts in each of the 1,024 channels. Usually, the channel number is converted to energy units (thousand electron volts ) by calibration. A typical EDXRF spectrum acquired with a gadolinium secondary target is shown in figure 1.

The intensity, or number of counts in a peak, is a direct result of the number of fluorescing atoms of that element in the sample; thus, the area under a peak is proportional to the concentration of that element in the sample. The relation of intensity to concentration is complicated by several factors. Because $\mathrm{X}$-rays produced in the sample originate from an extremely thin layer (micrometers to millimeters), the surface must be smooth and flat. 


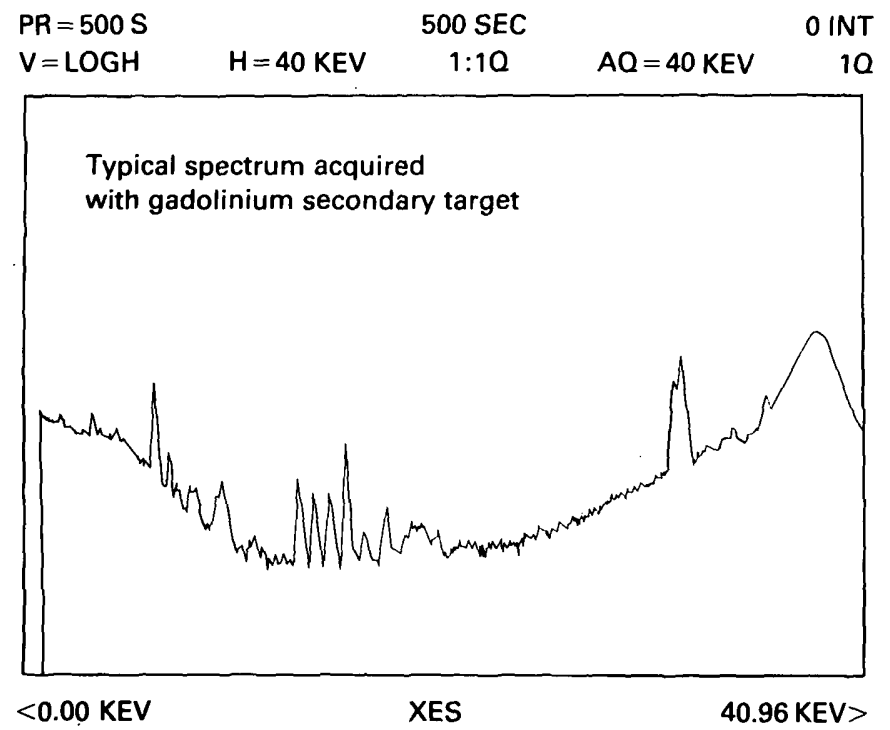

Figure 1. Typical energy-dispersive $X$-ray fluorescence spectrum with $X$-ray energy plotted with log of intensity.

The particle size distribution of the sample also affects intensity; as particle size increases, intensity decreases. These two problems usually can be resolved by adequate grinding and special sample preparation techniques.

The measured X-ray intensity also can be affected by interelement absorption and enhancement effects within the sample. The absorption effect originates because atoms within the sample matrix may absorb $\mathrm{X}$-rays fluoresced by other atoms before they reach the detector. The absorption effect causes measured intensity to be lower than expected. In general, the ability of an element to absorb X-rays increases with its atomic number. Similarly, the enhancement effect is caused by atoms that are excited not by the tube, but by X-rays from a neighboring atom. In this case, intensity is higher than expected. Unless corrected, absorption and enhancement can lead to serious errors in calculation of concentration from intensity data. Therefore, to produce accurate quantitative results from EDXRF (and WDXRF) data, the general requirements include not only adequate sample preparation and good standard reference materials, but also mathematical correction for matrix effects (absorption and enhancement).

\section{EXPERIMENTAL}

\section{Sample Preparation}

The most important requirements for samples presented to the spectrometer for X-ray analysis are that the specimen be homogeneous, flat, and smooth and that particle sizes be small enough so that further grinding produces no increase in intensity. Three types of specimens commonly are produced for $\mathrm{X}$-ray analysis-loose powders, briquettes, and fused disks. Loose powder and briquette preparations are discussed in greater detail by Bertin (1975), and fusion practices are discussed by Tertian and Claisse (1982).

For the loose powder preparation, about $1 \mathrm{~g}$ of ground rock powder (about 100 mesh) is poured into a cell formed by 0.00025 -in thick mylar stretched over a 1 -in diameter aluminum cylinder and held in place by a concentric plastic ring. This preparation method is fast and requires no weighing because neither binder nor flux is added. Because the method is completely nondestructive, samples can be returned unchanged (other than having been ground) to the submitter or subsequently analyzed for other elements that are not suited to measurement by EDXRF. Unfortunately, samples prepared in this way will have variable packing densities and are not free from particle size or mineralogical effects. Care must be taken either to minimize these problems or to find a mathematical correction for them.

To overcome the effects of variable sample packing densities, samples may be formed into briquettes. This is accomplished by the addition of a binder, such as cellulose, boric acid, or steric acid, followed by mechanical compression at pressures ranging from 10,000 to 40,000 $\mathrm{lb} / \mathrm{in}^{2}$. Although particle size and mineralogical effects are not eliminated completely by this procedure, it does produce samples that are all similar in density and that will last for a long period of time (several years) if kept in a dry atmosphere. Unfortunately, the briquetting process is time consuming, requiring several weighings and a substantial amount of mixing and grinding.

The third type of specimen to be used in the X-ray spectrometer is the fusion disk. The fusion process entails heating the sample with about 10 times its weight of a flux, such as lithium tetraborate, lithium metaborate, or sodium tetraborate (borax). The flux melts and reacts with or dissolves the sample so that the end product after cooling is a one-phase glass disk. Particle size and mineralogical effects are eliminated completely, and the density and matrix of all samples is similar. The procedure is ideal for almost every purpose, with a few exceptions. Some types of materials, such as whole coal, cannot be fused. Other materials, such as sulfide sulfur, may volatilize on fusion. Also, the 10:1 dilution, which normally is used for majorand minor-element analyses, is not practical for trace elements because the sample dilution reduces the sensitivity of the method. With the exception of these few cases, fusion is the method of choice for providing conditions with a high degree of homogeneity and the lowest interelement matrix effects.

\section{Quantitative Analysis}

To perform quantitative analysis with EDXRF (or WDXRF), it is always necessary to first optimize the 
sample preparation technique for the problem at hand and then to determine an adequate method of correction for absorption and enhancement. Matrix effects can almost always be reduced or eliminated through dilution. One of the advantages of the fusion procedure for sample preparation is that, with a tenfold dilution, the matrix of all samples is essentially identical to that of the flux. In this case, standards are prepared in the same way as the unknown specimens, and plots of intensity with concentration are usually linear, with very little scatter. Extremely large fluctuations in some constituents can cause absorption effects even in very dilute specimens. However, in the analysis of trace constituents, little dilution can be tolerated before detection limits and accuracy are degraded to an unacceptable level.

In cases where dilution cannot be used, it is necessary to find mathematical models for the correction of absorption and enhancement effects. Almost as many correction algorithms are available as X-ray spectroscopists, but, in general, three basic approaches are usedcalculations from fundamental parameters, interelement influence coefficients, and correction with scattered radiation.

The fundamental parameter method was developed by Criss and Birks (1968). The principle consists of assuming an approximate composition for the unknown specimen, calculating the fluorescence intensities from fundamental equations, and comparing with the measured intensities. Successive adjustments of the composition are made until the theoretical and the measured intensities are consistent. One advantage of this method is that only one standard actually is needed for an analysis.

The use of interelement influence coefficients for correction of absorption and enhancement is a widespread practice. Many versions of this method exist, but they all assign a coefficient to each element in a matrix to represent that element's effect on the measured intensity of the analyte element, as follows:

where

$$
C_{i}=R_{i}\left(1+\sum_{j=i}^{n} d_{i j} C_{j}\right),
$$

$$
\begin{aligned}
& i=\text { analyte element, } \\
& j=\text { all other elements in sample matrix, } \\
& n=\text { number of element in the sample matrix, } \\
& C_{i}=\text { concentration of element } i, \\
& R_{i}=\text { ratio of intensity from element } i \text { to intensity } \\
& \quad \text { from sample containing } 100 \text { percent } i, \\
& \quad \begin{array}{l}
\text { and } \\
d_{i j}=
\end{array} \\
& C_{j}=\text { confluence coefficient of element } j \text { for element } i, \\
&
\end{aligned}
$$

The coefficients can be calculated empirically from a set of multielement standards through a multiple leastsquares approach or from one of the many algorithms such as Lachance and Traill (1966), Rasberry and Hein- rich (1974), Lucas-Tooth and Pyne (1968), deJongh (1973), and so forth. Alternatively, the coefficients can be calculated from fundamental parameters as shown by Rousseau (1984). It is not surprising to note that, as dilution ratio increases, the effectiveness of most of these methods also increases.

The third class of correction methods, based on scattered radiation, was proposed by Andermann and Kemp (1958). The basis for this method is that, for a given concentration of some analyte, the intensities of the analyte radiation and primary radiation (from the excitation source) scattered by the specimen at a wavelength near the analyte line are affected in the same way by absorption of the sample. Therefore, their ratio should be almost independent of the matrix, and, in fact, this ratio has been found to be almost completely insensitive to excitation conditions, particle size, and the packing density of samples. In EDXRF analysis using secondary targets, it is usually possible to choose a secondary target that is very close to the analyte line. This not only optimizes the excitation of the element, but it also insures that the scatter from the source is close in energy to the analyte radiation. Thus, the scattered radiation correction approach has found wide usage for trace-element analysis (Johnson, 1984; Feather and Willis, 1976; Giaque and others, 1977). The method used in U.S. Geological Survey laboratories involves the use of ratio of analyte line intensity to secondary target scattered radiation. Calibration curves are prepared for each element by plotting this ratio against concentration for the appropriate standards.

The scatter method is applicable only to those elements with a higher atomic number than the major constituents. Also, the presence of an absorption edge between the analyte line and the scattered primary line (from excitation source) from an element whose concentration is greater than about 0.2 percent will cause calculated analyte results to be greater than they should be. This is caused by the "interfering" element affecting scattered radiation while not absorbing analyte radiation. The assumption that analyte and primary radiation are subject to the same absorption phenomena does not hold in this case, and an alternate method, or possibly an alternate secondary target, must be used.

\section{Standard Reference Materials}

The analysis of most major, minor, and trace elements is accomplished by comparison of unknown intensity to a calibration curve constructed by plotting concentration against "corrected" intensity for a set of standards. In general, as many standards as possible are used to construct this curve, and the 19 U.S. Geological Survey standards described by Flanagan $(1967,1976,1984)$ are almost always used for every element. Often, other inter- 
national standards are chosen to extend the ranges of this set. The values for the standard concentrations used for EDXRF analysis are those published by Abbey (1983), with the exception of basalt W-2, dunite DNC-1, and basalt BIR-1, where Flanagan's (1984) values are more comprehensive.

\section{Instrumentation}

The Geologic Division operates one Kevex 0700/7000 and one Kevex 0700/8000 EDXRF spectrometer in the Reston, Virginia, laboratory, and two 0700/7000 units in the Menlo Park, California, facility. Some effort has been exerted to maintain consistency between the two laboratories; analyses for the same suites of trace elements and sample preparation and absorption correction methods are identical. Thus, it is hoped that accuracy and precision are likewise similar, and data originating from either center should be unbiased with respect to the other.

The analytical work presently being performed by EDXRF includes several trace-element groups, each consisting of elements measured from a spectrum obtained by using a given secondary target. Barium, lanthanum, and cerium are determined by using a gadolinium secondary target and excitation conditions of $60 \mathrm{kV}$ and $2.0 \mathrm{~mA}$ with a 300-s acquisition time. Rubidium, strontium, yttrium, zirconium, and niobium constitute another group and originate from a spectrum excited by a silver secondary target at $40 \mathrm{kV}, 1.7 \mathrm{~mA}$, and a 300 -s acquisition time. Spectra from which nickel, copper, and zinc are determined are excited with a germanium secondary target at $35 \mathrm{kV}$ and $2.0 \mathrm{~mA}$ for $200 \mathrm{~s}$. Finally, a single element, chromium, is taken from a spectrum collected from an iron secondary target at $30 \mathrm{kV}, 2.0 \mathrm{~mA}$, and $200 \mathrm{~s}$. The loose powder sample preparation method and a correction based on scattered radiation, described above, are used for all trace-element determinations.

\section{SENSITIVITY, PRECISION, AND ACCURACY}

The precision of EDXRF measurements depends primarily on instrument stability, although it also can be influenced by variations in specimen preparation or by differences in sample geometry within the spectrometer. The particular spectrometers being used for EDXRF analysis at the Geological Survey are very stable, and single-intensity measurements are commonly reproducible to within a \pm 1 -percent relative error limited mainly by counting statistics. Accuracy (that is, the ability to reduce or eliminate systematic errors) depends on many factors. Nonuniform sample preparation, errors in standard reference material values, atomic number of analyte elements (lighter elements being affected by the matrix to a greater extent), the presence of interfering lines, and the ability of the correction algorithm to model the actual interelement effects contribute to the accuracy of a determination. Experience has shown that, with some diligence on the part of the analyst, a relative error of \pm 5 percent is common for most of the concentration ranges, although it decreases to as much as 50 percent near the lower limit of detection (about 2-10 ppm for most elements).

The detection limits depend on atomic numbers, background, X-ray line overlaps, excitation sources, and sample preparation methods. In principle, the upper limit for a determination is $\mathbf{1 0 0}$ percent, but, in many cases, standards defining this entire range are not available. When a particular result falls above the highest standard, that result is identified in the analytical report as having increased uncertainty. If necessary, the range for a particular standard can be extended by preparation of synthetic or secondary "standard" materials. The lower limit of detection and the upper limit of standard values currently in use are listed in table 1. These are subject to some variation due to changes in instrument performance over time.

Table 1. Lower limit of detection and upper limit of standard curve for typical energy-dispersive $\mathrm{X}$-ray fluorescence determinations [In parts per million]

\begin{tabular}{|c|c|c|}
\hline \multirow[b]{2}{*}{ Element } & \multicolumn{2}{|c|}{ Limit } \\
\hline & Lower & Upper \\
\hline $\mathrm{Cr}$ & 20 & 4,000 \\
\hline Mi & 2 & 3,000 \\
\hline $\mathrm{Cu}$ & 2 & 1,000 \\
\hline $2 n$ & 2 & 1.000 \\
\hline $\mathbf{R b}$ & 2 & 2.000 \\
\hline $\mathrm{Sr}$ & 2 & 2.000 \\
\hline$r$ & 2 & 500 \\
\hline $2 r$ & 5 & 2.000 \\
\hline Nb & 10 & 500 \\
\hline $\mathrm{Ba}$ & 5 & 4,000 \\
\hline La & 5 & 500 \\
\hline $\mathrm{Ce}$ & 5 & 500 \\
\hline
\end{tabular}




\section{REFERENCES CITED}

Abbey, Sidney, 1983, Studies in "standard samples" of silicate rocks and minerals, 1969-1982: Geological Survey of Canada Paper 83-15, 114 p.

Andermann, George, and Kemp, J. W., 1958, Scattered X-rays as internal standards in X-ray emission spectroscopy: Analytical Chemistry, v. 30, p. 1306-1309.

Bertin, E. P., 1975, Principles and practice of X-ray spectrometric analysis: New York, Plenum Press, 1079 p.

Criss, J. W., and Birks, L. S., 1968, Calculation methods for fluorescent X-ray spectrometry-empirical coefficients vs. fundamental parameters: Analytical Chemistry, v. 40, p. 1080-1086.

deJongh, W. K., 1973, X-ray fluorescence analysis applying theoretical matrix corrections-Stainless steel: X-Ray Spectrometry, v. 2, p. 151-158.

Feather, C. E., and Willis, J. P., 1976, A simple method for background and matrix correction of spectral peaks in trace element determination by X-ray fluorescence spectrometry: X-Ray Spectrometry, v. 5, p. 41-48.

Flanagan, F. J., 1967, U.S. Geological Survey silicate rock standards: Geochimica et Cosmochimica Acta, v. 31, p. 289-308.
1976, Descriptions and analyses of eight new USGS rock standards: U.S. Geological Survey Professional Paper 840, $192 \mathrm{p}$.

1984, Three USGS mafic reference samples, W-2, DNC-1, and BIR-1: U.S. Geological Survey Bulletin 1623, $54 \mathrm{p}$.

Giaque, R. D., Garrett, R. B., and Goda, L. Y., 1977, Energydispersive $\mathrm{X}$-ray fluorescence spectrometry for determination of 22 trace and 2 major elements in geochemical specimens: Analytical Chemistry, v. 49, p. 62-67.

Johnson, R. G., 1984, Trace element analysis of silicates by means of energy dispersive X-ray spectrometry: X-Ray Spectrometry, v. 13, p. 64-68.

Lachance,G. R., and Traill, R., 1966, A practical solution to the matrix problem in X-ray analysis, pt I, Method: Canadian Spectroscopy, v. 11, p. 43-48.

Lucas-Tooth, H. J., and Pyne, C., 1968, Accurate determination of major constituents by $\mathrm{X}$-ray fluorescent analysis in the presence of large interelement effects, in Advances in X-ray analysis: New York, Plenum Press, v. 7, p. 523-541.

Rasberry, S. D., and Heinrich, K. F. J., 1974, Calibration for interelement effects fluorescence analysis: Analytical Chemistry, v. 46, p. 81-89.

Rousseau, R. M., 1984, Fundamental algorithm between concentration and intensity in XRF analysis, pt 1, Theory: X-Ray Spectrometry, v. 113, p. 115-120.

Tertian, R. and Claisse, F., 1982, Principles of quantitative $\mathrm{X}$-ray fluorescence analysis: London, Heyden \& Sons, 385 p. 


\section{CHAPTER G}

Major and Minor Elements Requiring Individual Determination, Classical Whole Rock Analysis, and Rapid Rock Analysis

By L. L. JACKSON, F. W. BROWN, and S. T. NEIL

U.S. GEOLOGICAL SURVEY BULLETIN 1770

Methods for Geochemical Analysis 



\section{CONTENTS}

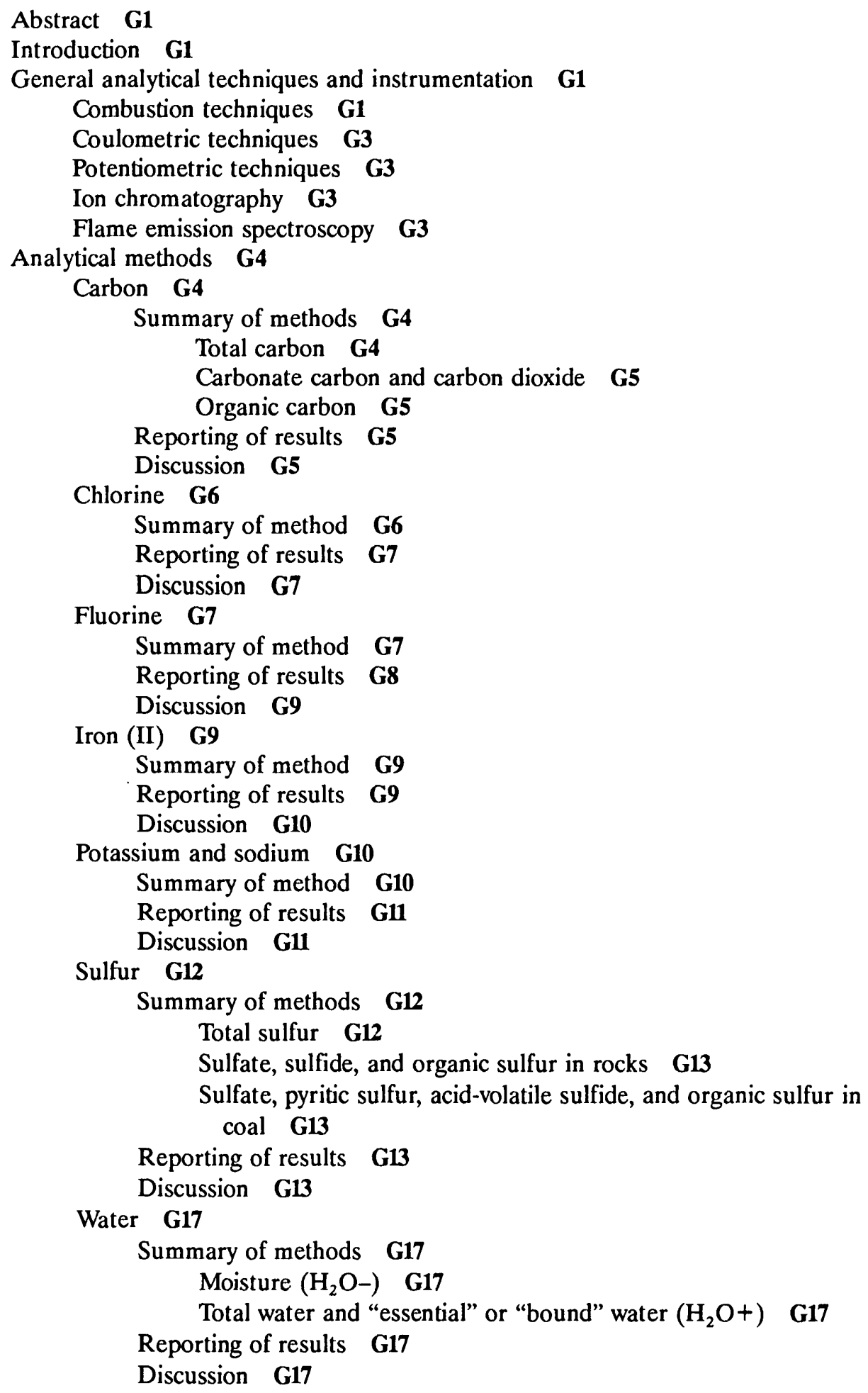


Classical whole rock analysis G19

Summary of methods $\mathbf{G 1 9}$

$\mathrm{H}_{2} \mathrm{O}-, \mathrm{SiO}_{2}, \mathrm{Al}_{2} \mathrm{O}_{3}, \mathrm{TiO}_{2}, \mathrm{Fe}_{2} \mathrm{O}_{3}, \mathrm{MgO}$, and $\mathrm{CaO} \quad \mathrm{G} 19$

$\mathrm{MnO}$ and $\mathrm{P}_{2} \mathrm{O}_{5} \quad \mathrm{G} 19$

$\mathrm{FeO}, \mathrm{Cl}, \mathrm{F}, \mathrm{Na}_{2} \mathrm{O}, \mathrm{K}_{2} \mathrm{O}, \mathrm{S}, \mathrm{CO}_{2}$, and $\mathrm{H}_{2} \mathrm{O}+\mathbf{G 1 9}$

Reporting of results G19

Discussion G19

Rapid rock major-element determination G20

Summary of methods $\mathbf{G 2 0}$

$\mathrm{SiO}_{2}, \mathrm{Al}_{2} \mathrm{O}_{3}, \mathrm{Fe}_{2} \mathrm{O}_{3}, \mathrm{MgO}, \mathrm{CaO}, \mathrm{Na}_{2} \mathrm{O}, \mathrm{K}_{2} \mathrm{O}, \mathrm{TiO}_{2}, \mathrm{P}_{2} \mathrm{O}_{5}$, and $\mathrm{MnO} \mathbf{G 2 0}$

$\mathrm{FeO}, \mathrm{Cl}, \mathrm{F}, \mathrm{S}, \mathrm{CO}_{2}$, and $\mathrm{H}_{2} \mathrm{O} \pm \mathbf{G 2 0}$

Reporting of results $\mathbf{G 2 0}$

Discussion G20

References cited G21

FIGURES

1. Chronological results between February 1983 and March 1985 for the determination of chlorine in the U.S. Geological Survey standard andesite AGV-1 G8

2. Chronological results for the determination of iron (II) oxide in U.S. Geological Survey standards andesite AGV-1 and Hawaiian basalt BHVO-1 G11

3. Paired chronological results between December 1983 and January 1985 for the determination of potassium in biotite control samples $\mathbf{G 1 2}$

\section{TABLES}

1. Summary of techniques used in the U.S. Geological Survey regional laboratories G2

2. Examples of calibration standards used for the CR-12 carbon analyzer

3. Summary data for the replicate analyses of $\mathbf{1 9}$ marine shales G6

4. Summary of potassium determinations by flame emission spectrometry obtained in the Menlo Park laboratory quality-assurance program G13

5. Sample size and calibration dependency of the apparent sulfur content in sulfide ore SU-1 G14

6. Forms of sulfur by different speciation techniques G16

7. Organic carbon-organic sulfur ratios in green river shale SGR-1 and marine sediment MAG-1 G17

8. Direct determination of water + in mica-magnesium and mica-iron standards by Karl Fischer titration $\mathbf{G 1 8}$

9. Examples of the precision for the classical whole rock analysis $\mathbf{G 2 0}$

10. Estimate of relative precision for rapid rock analysis G21 


\title{
Major and Minor Elements Requiring Individual Determination, Classical Whole Rock Analysis, and Rapid Rock Analysis
}

\author{
By L. L. Jackson, F. W. Brown, and S. T. Neil
}

\begin{abstract}
Methods for the determination of $\mathrm{FeO}$; forms of $\mathrm{H}_{2} \mathrm{O}, \mathrm{C}$, and $\mathrm{S}$; $\mathrm{Cl}$; and $\mathrm{F}$ are described. These constituents are determined individually in rock samples to characterize their major- and minor-element composition, in addition to the determination of 10 major elements by X-ray fluorescence spectroscopy. Alternative chemical methods for determining the 10 major elements in rock samples, generally referred to as classical whole rock and rapid rock analyses, also are described briefly. A summary of each determination, a discussion of potential problems encountered with the methods or particular sample types, and example data on reference samples are included.
\end{abstract}

\section{INTRODUCTION}

The main purpose of this chapter is to describe the determinations for major and minor rock constituents ( $>0.01$ percent) that are done by techniques other than $\mathrm{X}$-ray fluorescence analysis. These constituents, which usually are determined one at a time, include $\mathrm{FeO}, \mathrm{H}_{2} \mathrm{O}$, forms of carbon, forms of sulfur, $\mathrm{Cl}, \mathrm{F}, \mathrm{Na}_{2} \mathrm{O}$, and $\mathrm{K}_{2} \mathrm{O}$. Also included in this chapter are brief descriptions of classical whole rock analysis and rapid rock analysis for $\mathrm{SiO}_{2}, \mathrm{Al}_{2} \mathrm{O}_{3}, \mathrm{Fe}_{2} \mathrm{O}_{3}, \mathrm{MgO}, \mathrm{CaO}, \mathrm{TiO}_{2}, \mathrm{P}_{2} \mathrm{O}_{5}$, and $\mathrm{MnO}$, as well as the constituents listed above.

This chapter is divided into two sections in which the types of analytical techniques and the specific methods used to determine the individual constituents are described briefly.

\section{GENERAL ANALYTICAL TECHNIQUES AND INSTRUMENTATION}

The classic texts by Washington (1904), Hillebrand (1919), Hillebrand and others (1953), Groves (1951), and Peck (1964) describe laborious gravimetric, titrimetric, and colorimetric procedures for rock analysis. Many of the classical techniques have been replaced by instrumental techniques that generally are easier and faster and offer lower limits of detection. However, they are frequently no more precise.

Combustion techniques with infrared (IR) or thermal conductivity (TC) detectors now are being used to determine total carbon, sulfur, and water. Water also is determined by combustion followed by a Karl Fischer coulometric titration, which replaces the Penfield (1894) gravimetric method. Acid-evolved carbon dioxide also is determined by coulometric titration. Potentiometric techniques [ion-selective electrodes (ISE)] and ion chromatography (IC) have changed greatly the way halogens and a variety of other anions are determined. The J. Lawrence Smith (1871) gravimetric method for alkali metals, which was used routinely for almost $100 \mathrm{yr}$ has been replaced by flame emission spectrometry (FES). These changes in methodology have occurred not only due to improvements in technology, but to meet the ever-increasing demand for geochemical analyses.

The techniques used for the determination of the different constituents vary among the three regional laboratories depending upon the instrumentation available, number and type of samples, quantity of sample, geologist's needs, and analyst biases. Table 1 summarizes the general techniques used routinely in the regional laboratories.

\section{Combustion Techniques}

In combustion techniques, the samples usually are heated, typically to $1,000-1,600^{\circ} \mathrm{C}$, in a high-frequency induction furnace or a more conventional resistance tube furnace under an oxygen atmosphere. A combustion aid or accelerator, such as copper, vanadium pentoxide, or tungsten oxide, frequently is mixed with the sample. Specific combustion products are detected with IR or TC detectors. The IR detector is a solid-state nondispersive detector used to determine a single component in a gas 
Table 1. Summary of techniques used in the U.S. Geological Survey regional laboratories

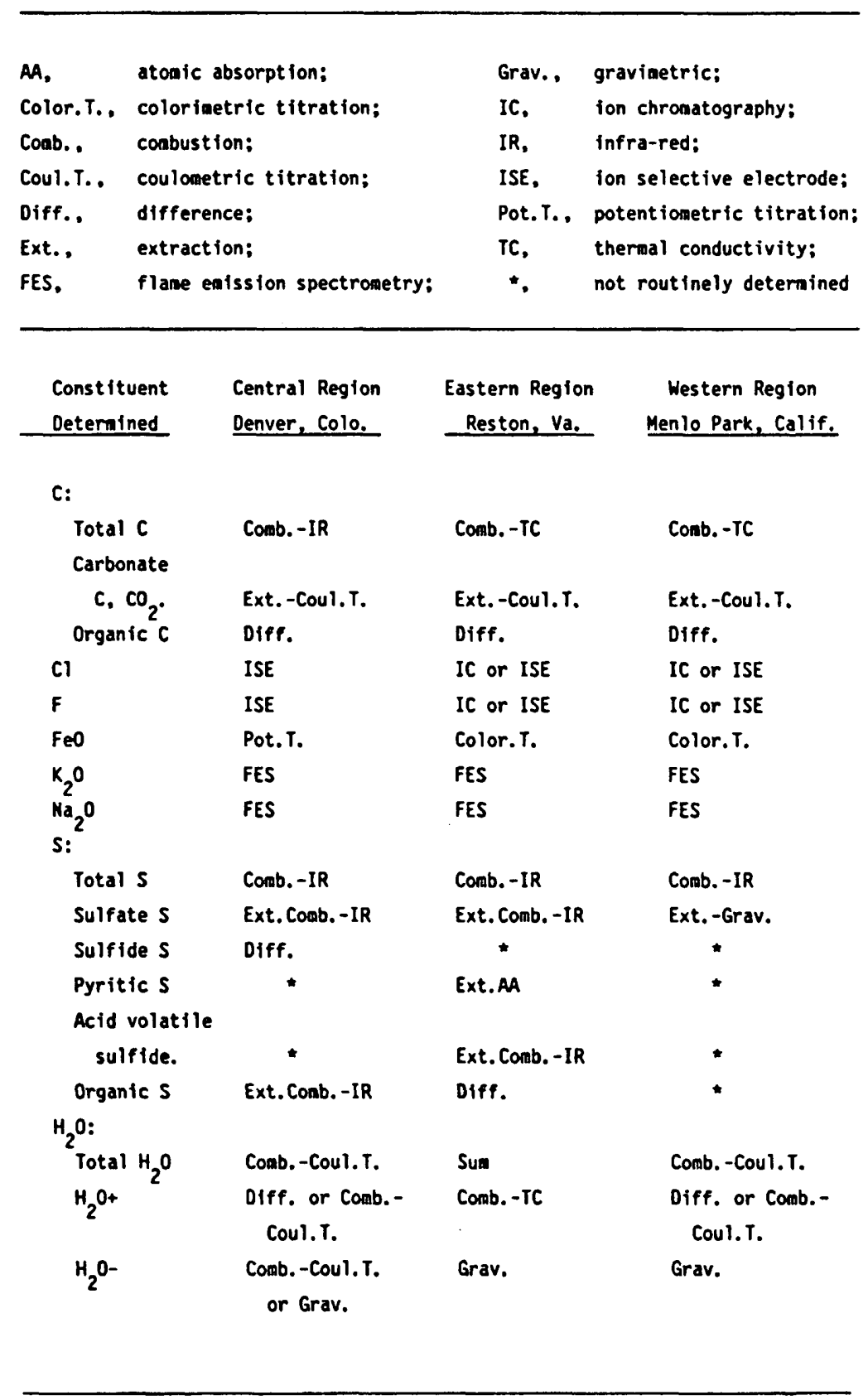

stream. Two problems encountered with this type of detector are overlap of IR absorption spectrum from several components in the gas stream and nonlinear relation between concentration and absorbance. These problems are overcome by stripping interfering components from the gas stream before detecting the species to be determined, or analyte, and by calibrating the instrument with standards that closely approximate the analyte concentration and sample matrix.

Thermal conductivity detectors consist of a heated wire filament or a thermistor whose resistance varies based on the thermal conductivity of the flowing gas stream. The detectors usually are paired as part of a Wheatstone bridge to discriminate against variations in experimental conditions and the thermal conductivity of the carrier gas. Sensitivity of the detector is a function of the difference in thermal conductivity of the analyte species and the carrier gas. Because the detector is nonselective, interfering species must be stripped from the carrier gas. In the typical automated elemental $(\mathrm{C}, \mathrm{H}$, $\mathrm{N})$ analyzer, total thermal conductivity of the gas stream is measured, carbon dioxide is removed, thermal conduc- 
tivity is measured again, water is removed, and thermal conductivity is measured a third time. Thus, specificity of these difference measurements is controlled by the ability of the stripping reagents to remove selected species.

\section{Coulometric Techniques}

In a coulometric titration, because the titrating reagent is generated electrolytically, usually under constant current conditions, the analyte does not undergo direct reaction at the electrode. If the titrant reacts stoichiometrically with the analyte and if the current is 100 -percent efficient during titrant generation, then the product of current and time is proportional to the analyte concentration. The endpoint of the titration can be detected colorimetrically, potentiometrically, or by a variety of other means. In the determination of carbon dioxide, a base is generated coulometrically, and the titration endpoint is determined colorimetrically, but, in the Karl Fischer water determination, the iodine titrant is generated coulometrically, and a potentiometric endpoint is used.

The coulometric titration offers several advantages over the conventional volumetric titration-current and time can be measured more accurately than volume, no reagent standardization is required, a wide concentration range can be determined, and the titrations are generally rapid and easily automatable. The coulometric titration suffers from the same problems of specificity of the titration and endpoint detection as volumetric methods.

\section{Potentiometric Techniques}

In potentiometry, the measured potential between an indicator electrode and a reference electrode is related to the analyte concentration. The determination of $\mathrm{pH}$ with a glass electrode is the most common use of an ISE for direct potentiometric measurement. Other direct methods include the use of solid-state ISE's for the determination of anions, such as chloride and fluoride. An ISE measures the free-ion activity in a solution that depends on ionic strength, complexing agents, and so forth, and not necessarily the total ion concentration. The selectivity of an ISE is dependent upon the ion-exchange properties of the membrane. Major interferences for the solid-state electrodes are usually ions that form more insoluble salts with the membrane counter ion than does the analyte ion; for example, the chloride ISE membrane is made of mixed silver sulfide-silver chloride salts. Bromide, iodide, sulfide, cyanide, and ammonia interfere with the chloride determination. The magnitude of their interference is dependent upon their solubility product or complexation constants. The lower limit of detection, which is fre- quently $10^{-5}-10^{-6} \mathrm{M}$, also is determined by the solubility of the salts.

Potentiometric indicator electrodes, such as platinum or silver metal, can be used for endpoint detection in volumetric methods. Potentiometric titrations are inherently more accurate than direct potentiometric measurements; however, they are usually slower. In a direct determination of a monovalent ion, a $2-\mathrm{mV}$ error in potential measurement would represent an 8-percent uncertainty in the ion concentration; however, in a potentiometric titration, this same error in the determination of the equivalence point usually would represent a far smaller uncertainty. Also, in the titration, the amount of analyte in solution is determined, as opposed to its concentration.

\section{Ion Chromatography}

Ion chromatography is the separation of ions using a solid ion-exchange resin and the detection of these ions in a flowing stream. A conductivity detector is used most frequently. Although many aspects of this technique have been utilized for years, the recent introduction of commercial, automated instrumentation has revolutionized this technique. The advent of this new instrumentation has permitted the determination of several anions or cations in solution in a matter of minutes. Its major disadvantage in the geochemical laboratory is the difficulty of digesting geologic samples without adding significant quantities of interfering ions to the sample solution.

Although the technology has advanced in recent years, IC as practiced in the Geologic Division of the U.S. Geological Survey is very similar to the original technique described by Small and others (1975). In this technique, two ion-exchange columns and a conductivity detector are used. The first column separates the analytes, and the second column lowers the conductivity of the eluent allowing the analyte ions to be detected with minimal background conductivity. In the determination of anions, where the eluent is a mixture of carbonate species, a hydrogenated strong cation-exchange resin is used in the second column. Thus, the eluent is converted to $\mathrm{H}_{2} \mathrm{CO}_{3}$, which has low conductivity. If the acid dissociation constants for the anions of interest are less than $10^{-7}$, then no analyte conductivity will be detected. Fluoride, chloride, phosphate, nitrate, sulfate, and bromide are anions typically determined by IC. In aqueous samples detection limits of $0.1 \mathrm{ppm}$ are not uncommon (Fishman and Bradford, 1982). A description of IC also can be found in Chapter D.

\section{Flame Emission Spectrometry}

A sample solution is aspirated into a propane-air flame. A monochromator or filter is used to isolate the 
light emitted by the analyte atoms, and a photomultiplier tube is used to detect the emitted light. Variations in flame characteristics and sample introduction frequently are overcome by ratioing the analyte signal to that of an internal standard.

\section{ANALYTICAL METHODS}

In this section, specific methods utilized by the U.S. Geological Survey regional laboratories are outlined. The symbols [D], $[M]$, and [R] are included throughout the text to designate specific procedures used by the individual laboratories-Central Region, Denver, Colorado; Western Region, Menlo Park, California; and Eastern Region, Reston, Virginia; respectively. The purpose of this section is not to provide a step-by-step procedural manual, but to give a brief summary of the methods used and a discussion of these methods focused largely on the limitations and problems associated with specific procedures or samples. More comprehensive treatises on silicate analysis should be consulted for comparison of methods, additional procedural details, and limitations of the techniques (Groves, 1951; Hillebrand and others, 1953; Johnson and Maxwell, 1981; Jeffery and Hutchison, 1981); the Menlo Park laboratory also has published a manual containing many of the currently used step-bystep procedures (Cremer and others, 1984).

The main points of each method summary are the general technique, sample size routinely used, digestion technique, and type of instrumentation (including manufacturer where necessary for clarification of the technique). The protocol for reporting results also is included. The lower limit for reporting of results is defined here as limit of determination. The limit of determination frequently is related more closely to historical practice than to a rigorously defined limit of detection (Long and Winefordner, 1983). It should be stressed that the summaries describe the routine procedures that generally were developed for the analysis of igneous silicates. Modifications frequently are made to meet special requirements for the analysis of different matrices, sample sizes, and (or) concentration ranges.

In the discussion section for each constituent, the limitations of the technique and problems associated with specific sample types, often relatively rare minerals, are emphasized. All too often, the sample description provided by the geologist is inadequate to alert the analyst to sample or to samples that are not appropriate for a particular technique. Also, without an understanding of the limitations of the analytical methods inappropriate geochemical interpretations may be made.

In an effort to convey a sense of the error associated with these measurements, a small amount of data, which problems that will be encountered with a particular

emphasizes the analytical precision, has been included for each constituent. Most of these data have been obtained in the Denver laboratory from the analysis of Geological Survey geochemical standards that are more finely ground and better homogenized than the average sample. Obviously these data may be a poor estimate of what will occur when analyzing "real world" samples, which are different types of material, such as igneous and sedimentary, or even within a class, such as granites and basalts. The precision obtained is dramatically concentration dependent in many instances. Accuracy has not been emphasized because it is a difficult term to define in rock analysis. Where appropriate, some comparisons have been made to Abbey's (1983) "usable" values, which are based on the concensus of data among selected international geochemical laboratories. Unless otherwise specified, Abbey's usable values are reported on a dry-weight basis, and all other values reported herein are on an as-received basis.

\section{Carbon}

\section{Summary of Methods}

\section{Total Carbon}

$[\mathrm{D}, \mathrm{M}]$
A Leco CR-12 automated carbon analyzer with a resistance furnace and IR carbon dioxide detector is used for samples with carbon content ranging from 0.01 to about 30 percent. The sample of about $0.75 \mathrm{~g}$ is combusted in an oxygen atmosphere at $1,370^{\circ} \mathrm{C}$. Aluminum oxide (about $1 \mathrm{~g}$ ) is added to the more carbonaceous samples to slow down the evolution of carbon dioxide and to prevent saturation of the infrared cell. Because the infrared cell response is nonlinear, the calibration standard is chosen to most closely represent the carbon content of the sample, as shown in table 2 .

A Leco WR-12 carbon analyzer with an induction furnace and a TC detector is used (Tillman, 1977; Cremer and others, 1984). Copper metal [D] or copper and iron [M] are used as an accelerator. Up to a 1-g sample is analyzed for percent carbon of less than 5 percent. Above 5 percent $C$, the sample size is decreased proportionately.

A Perkin-Elmer 240B automated elemental $(\mathrm{C}, \mathrm{H}, \mathrm{N})$ analyzer is used to determine total carbon and $\mathrm{H}_{2} \mathrm{O}+$ on predried samples (Din and Jones, 1978; Nelson and Sommers, 1982; Kirschenbaum, 1983). The sample, usually $15-20 \mathrm{mg}$, is combusted at $1,000^{\circ} \mathrm{C}$. 
Table 2 . Examples of calibration standards used for the CR-12 carbon analyzer

\begin{tabular}{|c|c|c|c|c|}
\hline \multirow{2}{*}{\multicolumn{2}{|c|}{$\begin{array}{c}\text { Sample } \\
\text { C content } \\
\text { (percent) }\end{array}$}} & \multirow[b]{2}{*}{$\begin{array}{c}\text { Sample } \\
\text { size } \\
(g)\end{array}$} & \multicolumn{2}{|c|}{ Calibration standard } \\
\hline & & & Type & Percent C \\
\hline & $1-1$ & 1 & Shale, SCo-1 & $0.99^{\mathrm{d}}$ \\
\hline 1 & -5 & 0.75 & Soil, $50-4$ & $4.40^{b}$ \\
\hline 5 & -12 & 0.50 & Dolomite, $\mathrm{NBS}^{1} 88 \mathrm{a}$ & $12.72^{c}$ \\
\hline & 12 & 0.25 & 011 shale, SGR-1 & $27.5^{d}$ \\
\hline
\end{tabular}

1 HBS: National Bureau of Standards.

Abbey, 1983.

bowman and others, 1979.

${ }^{c}$ Based on NBS certificate of analys is $\mathrm{CO}_{2}$ content.

dValue determined in-house.

\section{Carbonate Carbon and Carbon Dioxide}

[D,M,R] The sample is digested with $2 M$ perchloric acid, and the carbon dioxide evolved is collected in a coulometric cell, where it is converted to a strong titratable acid by ethanolamine. The acid is titrated automatically with a coulometrically generated base and the endpoint is detected colorimetrically (Norton and Engleman, 1980; Engleman and others, 1985). The sample size is varied depending upon the carbon content $-0.5 \mathrm{~g}$ sample per 0.01-5 percent $\mathrm{CO}_{2}, 0.1 \mathrm{~g}$ sample per 5-10 percent $\mathrm{CO}_{2}$, and $0.02 \mathrm{~g}$ sample per $>10$ percent $\mathrm{CO}_{2}$.

[M,R] For samples containing 0.1 percent $C$ or less, total carbon, determined as described above, is reported as carbonate carbon.

\section{Organic Carbon}

$[D, M, R] \quad$ Organic carbon is not determined directly but is computed from the difference of the total carbon and carbonate carbon determinations.

\section{Reporting of Results}

Total carbon, organic carbon, and carbonate carbon are reported as percent $C$ with a limit of determination of 0.01 percent $C[D, R]$. Total carbon $[M]$ and carbonate carbon $[D, M, R]$ also are reported as percent $\mathrm{CO}_{2}$ with the same limit of determination.

\section{Discussion}

Carbon in geologic materials is found as carbonates and carbonaceous matter, which includes organic matter and graphite. Total carbon is determined by combustion of the sample and quantitation of the evolved carbon dioxide, whereas carbonate carbon is quantitation of the acidliberated carbon dioxide. Several problems are encountered in liberating the carbon dioxide for both determinations.

Anhydrous alkaline earth carbonates are quite temperature stable with stability increasing with ionic radius of the metal (Todor, 1976). Magnesite decomposes completely at about $900^{\circ} \mathrm{C}$. The rarer strontium and barium carbonates, strontianite and witherite, require temperatures of about $1,200^{\circ} \mathrm{C}$ for complete decomposition. The Leco combustion equipment used for the total carbon determination approaches $1,400^{\circ} \mathrm{C}$ and $1,600^{\circ} \mathrm{C}$ for the resistance and induction furnaces, respectively; in the $\mathrm{C}$, $\mathrm{H}, \mathrm{N}$ analyzer, combustion occurs at $950^{\circ} \mathrm{C}$. Although the lower temperature is suitable for most samples that contain carbon, the presence of the more stable, but rarer, carbonates would require the use of a flux, such as vanadium pentoxide.

A variety of acids commonly are used to liberate carbon dioxide from carbonates. Hydrochloric acid is recommended most frequently; however, volatilized chloride would interfere in the coulometric titration. Dilute perchloric acid is used instead. Engleman and others (1985) obtained excellent results for National Bureau of Standards argillaceous and dolomitic limestones by using perchloric acid. Reasonable comparisons with Abbey's (1983) usable values for a variety of silicate standards also were obtained. Several sample types may present problems for acid-evolution methods. Hillebrand (1919) noted that scapolites were difficult to decompose with hydrochloric acid, and Peck (1964) found that siderite, in particular, required heating. Engleman and others (1985) found that perchloric acid attacked shales very slowly, often requiring $30-40 \mathrm{~min}$ for complete liberation of the carbon dioxide. The carbonate grains appeared to be coated with organic matter, which retarded carbon dioxide evolution. The addition of a wetting agent improved the speed of decomposition. No evidence of decomposition of the organic matter itself, resulting in high carbonate values, was found. 
The determination of total carbon and of carbonate carbon have potential interferences, mainly sulfur, chlorine, and fluorine. In both determinations, concentrations of these elements commonly found in silicates or carbonates are removed by suitable trapping agents, such as manganese dioxide, in the Leco WR-12 carbon analyzer or silver sulfate solution in the coulometric titration. Large concentrations of these interferences may exhaust the traps and, thus, require a reduction in sample size to avoid interference. This is particularly true of fluorine when using the Leco CR-12 for total carbon determination. Fluorine, when greater than 0.2 percent, has a deleterious effect on the instrumentation that requires a reduction in sample size or use of the Leco WR-12 carbon instrument.

Relatively little work has been done to determine the total carbon content of international geochemical standards. Abbey (1983) only listed usable values for 17 standards, 11 of which were questionable. Adequate standards covering a wide range of concentrations and sample matrices are lacking. This is a particular handicap when using an IR detector for carbon, as in the Leco CR-12 analyzer. The best results are obtained when the carbon concentration and the matrix of the calibration standard closely match the sample. This avoids difficulties due to nonlinear response of the detector, which is corrected mathematically based on a one-point calibration and is due to different rates of carbon release between the standard and the sample.

The triplicate analysis of 21 calcareous and siliceous marine shales for total carbon using the Leco CR-12 analyzer gave relative standard deviations of less than 2 percent for carbon in the range between 3 and 10 percent (L. L. Jackson and E. E. Engleman, U.S. Geological Survey, written commun., 1985). Flanagan and others (1976) obtained relative standard deviations of 12-20 percent by using a $\mathrm{C}, \mathrm{H}, \mathrm{N}$ analyzer to determine carbon in three Geological Survey volcanic rock standardsColumbia River basalt BCR-1, 0.005 percent C; Hawaiian basalt BHVO-1, 0.008 percent $\mathrm{C}$; and rhyolite RGM-1, 0.004 percent C. Engleman and others (1985) analyzed 12 Geological Survey rock standards for carbonate carbon and obtained relative standard deviations of 1-5 percent and about 0.5 percent for samples containing $0.01-2$ percent $\mathrm{CO}_{2}$ and $>2-47$ percent $\mathrm{CO}_{2}$, respectively.

A variety of procedures has been used to determine organic carbon directly. However, many of these procedures use an acid-leached sample that may have significant loss of soluble, hydrolyzable, or volatile organic compounds due to leaching (Leventhal and Shaw, 1980). Thus, organic carbon determined by difference includes all forms of carbonaceous matter, including graphite. The precision (standard deviation) of the difference measurement can be estimated as the square root of the summa- tion of the variances for total carbon and carbonate determinations. A summary of data from replicate analyses of 19 marine shales is shown in table 3 . On an absolute basis, the standard deviation for the determination of organic carbon was slightly larger than that for total carbon, and the relative standard deviation averaged about 2 percent.

\section{Chlorine}

\section{Summary of Method}

[D,M,R] The sample $(0.2 \mathrm{~g})$ is digested overnight in the outer ring of a sealed Conway diffusion cell with $\mathrm{KMnO}_{4}, \mathrm{H}_{2} \mathrm{SO}_{4}$, and HF. Chlorine is distilled from the outer chamber and reduced to chloride in the inner chamber, which contains $\mathrm{Na}_{2} \mathrm{SO}_{3}$ and $\mathrm{KOH}$. The chloride is measured directly in the inner chamber with a chloride ISE (Aruscavage and Campbell, 1983).

[M] Chloride is determined colorimetrically in an aliquot of a water leach of a sample $(0.1 \mathrm{~g})$ sintered with $\mathrm{Na}_{2} \mathrm{CO}_{3}$ and $\mathrm{ZnO}$ (see $\mathrm{F}$ determination). Ferric nitrate and mercury thiocyanate are added to the leach solution. The absorbance of ferric thiocyanate at $465 \mathrm{~nm}$ is proportional to the chloride concentration (Huang and Johns, 1967; Cremer and others, 1984).

[R] Chloride is determined simultaneously with fluoride by IC. The $100-\mathrm{mg}$ sample is

Table 3. Summary data for the replicate $(n=2-4)$ analyses of 19 marine shales

[In percent carbon, unless otherwise noted. L.L. Jackson and E.E. Engleman, U.S. Geological Survey, written commun., 1985; SD: Standard Deviation; RSD: Relative Standard Deviation (percent)]

Total C Organic C Carbonate

\begin{tabular}{|c|c|c|c|}
\hline Range & -17 & -13 & $0.01-10$ \\
\hline Mean & 10 & 6.2 & 3.8 \\
\hline Range of SD & $0.01-0.2$ & $0.02-0.2$ & $0.001-0.08$ \\
\hline Mean SD & 0.07 & 0.08 & 0.003 \\
\hline Mean RSD & 0.7 & 1.6 & 2.0 \\
\hline
\end{tabular}


fused with $200 \mathrm{mg}$ of $\mathrm{Na}_{2} \mathrm{CO}_{3}$. The water leach of the carbonate fusion cake is injected into the ion chromatograph with a conductivity detector (Wilson and Gent, 1983).

\section{Reporting of Results}

Chlorine is reported as percent $\mathrm{Cl}$ with the limit of determination varying by procedure-ISE, 0.01 percent; colorimetric, 0.1 percent; and IC, 0.0025 percent.

\section{Discussion}

Although chloride is common in most geologic materials, it usually is present at less than 0.1 percent. The major obstacle in determining chloride at this level is digesting the sample such that a detectable quantity of chloride is obtained in solution without major interferences. In the Reston laboratory, Aruscavage and Campbell (1983) developed a technique in which the chloride is distilled from the sample and is collected without significant dilution. They used a Conway diffusion cell made of teflon, which is very similar to the polyethylene cell used by Greenland $(1962,1963)$ for the determination of fluoride in solutions and chloride in meteorites. In Greenland's work, the halogens were determined colorimetrically, whereas Aruscavage and Campbell used a chloride ISE.

The Conway cell is a sealed system with an inner and outer chamber formed by two concentric rings with a conjoined gas space. In the outer chamber, the sample is decomposed by the acids, and chloride is oxidized to chlorine gas, which diffuses to the inner chamber where it is reduced to chloride in solution. The chloride then is determined in the inner chamber. Although 100 percent of the chloride is not transferred to the inner chamber [Aruscavage and Campbell (1983) found 90-percent recovery], if the calibration standards and samples are treated alike, chloride results that compare well with those determined by other methods are obtained.

Two problems may occur with the diffusion procedure. First, samples with unusually high amounts of organic matter, iron (II), or sulfide species may use up the oxidizing power of the digestion solution before evolution of the chloride. Second, the hydrofluoric and sulfuric acid digestion may not release all the chloride from some matrices; for example, apatites and sodalites easily release chloride, and the scapolite group does not.

In the colorimetric and IC procedures, the sample is sintered or fused, respectively, with sodium carbonate. Most chloride-bearing minerals are digested suitably in this fashion. However, phosphates and possibly some carbonates high in calcium and fluoride may have chloride occluded in the insoluble residue. This would be more of a problem in the fusion than in the sinter.
Chloride also may be volatilized if the sinter or fusion is too long or the temperature is too high (Hillebrand and others, 1953). Mercury chloride salts are particularly susceptible to volatilization.

Bromine and iodine behave similarly to chloride in the ISE and colorimetric determinations of chloride. However, because their natural abundance is much lower than chloride, they usually do not present a problem. A variety of anions may interfere with the IC procedure. Because it is a serial elution of several anions, any anion that is present in high concentration relative to chloride and elutes either before or after chloride may mask the chloride. Thus, fluorapatite or gypsum may pose problems due to fluoride eluting before and phosphate and sulfate eluting after chloride. In the colorimetric procedure, unoxidized sulfide in high concentration may interfere during the sinter.

Aruscavage and Campbell (1983) analyzed 30 geologic standards by the ISE technique. They generally found excellent agreement with other literature values for these standards. Figure 1 shows the chloride values determined over a 2-yr period for Geological Survey standard andesite AGV-1 in the Denver laboratory by this technique. The mean of $0.012 \pm 0.002$ percent $\mathrm{Cl}( \pm 1 \sigma)$ compares well with the mean of $0.0119 \pm 0.0005$ percent $\mathrm{Cl}$ obtained by Aruscavage and Campbell and with Abbey's (1983) usable value of 0.010 percent $\mathrm{Cl}$. The relative standard deviation for the 19 values obtained over the extended period was 18 percent; Aruscavage and Campbell had a relative standard deviation of 4 percent $(n=9)$ over a short time period. In general, they obtained relative standard deviations of less than 8 percent for samples with chloride contents ranging from 0.002 to 0.05 percent $\mathrm{Cl}$. It should be noted that for a sample size of 0.2 $\mathrm{g}$, the limit of determination is about 0.01 percent without venturing into the non-Nernstian (nonlinear) portion of the calibration curve.

The colorimetric procedure offers similar precision to the ISE technique (Huang and Johns, 1967); however, the limit of determination of the former is higher $(0.1$ percent $\mathrm{Cl}$ ). In the Denver laboratory, Wilson and Gent (1983) analyzed 21 Geological Survey rock standards by IC and obtained relative standard deviations generally better than 8 percent for samples with chloride content ranging from 0.002 to 3 percent $\mathrm{Cl}$. They estimated a limit of detection of 0.0007 percent ( $7 \mathrm{ppm}$ ); however, the limit of determination normally used is 0.0025 percent.

\section{Fluorine}

\section{Summary of Method}

Rock samples $(0.025 \mathrm{~g})$ are fused with sodium hydroxide, and the fusion cake is dissolved in water. The basic solution is buffered 


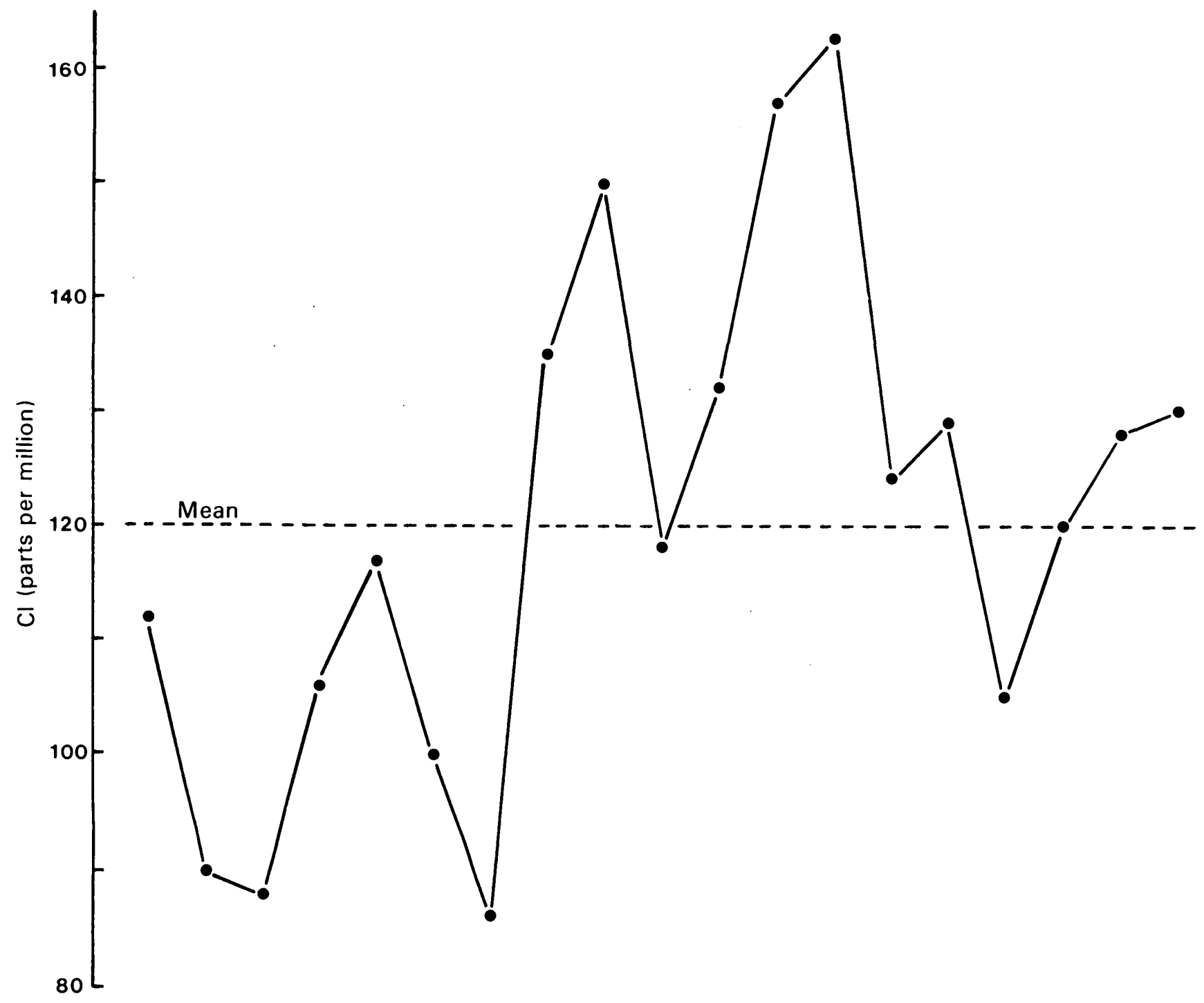

Figure I. Chronological results between February 1983 and March 1985 for the determination of chlorine in the U.S. Geological Survey rock standard andesite AGV -1 [mean $=120 \mathrm{ppm}$ chlorine, standard deviation $=20$ ppm chlorine (as-received basis), usable value (Abbey, 1983) $=100 \mathrm{ppm}$ (dry-weight basis)].

with ammonium citrate to about $\mathrm{pH} 6$. The fluoride is determined with an ISE by using a calibration curve (Hopkins, 1977). Coal samples $(0.25 \mathrm{~g})$ undergo a preliminary ashing with magnesium oxide and magnesium nitrate. The ash then is analyzed in the same way as a rock sample.

[M] The sample $(0.08 \mathrm{~g})$ is fused with lithium metaborate, and the fusion cake is dissolved in dilute nitric acid. A complexing agent and buffer [1,2-diaminocyclohexene-N,N,N,'N'tetraacetic acid (DCTA) and sodium citrate] are added. The fluoride is determined with an ISE using standard additions (Bodkin, 1977; Cremer and others, 1984).

[M,R] Fluoride and chloride are determined in a procedure in which the sample $(0.1 \mathrm{~g})$ is sintered with sodium carbonate and zinc oxide and then leached with water [M]. Fluoride is determined by ISE in a sodium citrate-potassium nitrate buffered aliquot of the acidified leach solution (Huang and Johns, 1967; Ingram, 1970; Cremer and others, 1984) $[M, R]$. Chloride is determined colorimetrically in a separate aliquot of the leach solution [M].

[R] Fluoride and chloride are determined sequentially by IC in the water leach from a sample $(0.1 \mathrm{~g})$ fused with sodium carbonate (2:1 flux to sample) (Wilson and Gent, 1982).

\section{Reporting of Results}

Fluorine is reported as percent $F$ with a limit of determination for the ISE procedures of 0.01 percent for 
rocks [D,M,R] and 0.002 percent for coals [D]. The limit of determination for the IC procedure is 0.0025 percent [R].

\section{Discussion}

Fluorine commonly is found in rocks at less than 0.1 percent; however, it is associated with, or an essential constituent of, fluorite, cryolite, topaz, apatites, tourmalines, titanite, humites, pyrochlores, micas, and amphiboles. Biotite, phlogopite, lepidolite, zinnwaldite, and chondrodite may contain fluoride in excess of 1 percent. Many of the rock-forming minerals listed are particularly difficult to digest, hence the use of sodium hydroxide or lithium metaborate fusion in the ISE procedure for fluoride. Also, in these fusions, calcium fluoride does not remain as part of an insoluble residue. With the sodium carbonate sinter or fusion for carbonates or phosphates, calcium fluoride may be insoluble without an acid leach of the digestion cake. Hillebrand and others (1953) stated, “...certain fluorides and phosphates are not completely decomposed so as to permit aqueous extraction of the anion unless silica is incorporated with the flux and mineral powder before the fusion is made." They also suggested that pyrite causes loss of fluoride during fusion. Certainly too long or too hot a fusion with carbonate promotes volatilization of fluorine.

Nicholson (1983) reviewed the problems associated with the ISE determination of fluoride in geological materials including practical experimental errors, limitations of the potentiometric sensor, and interfering species. Interference due to aluminum and iron complexation of fluoride generally are avoided by the addition of a masking agent. In the ISE procedures outlined above, citrate is added as a buffer and as a masking agent; DCTA also is added as a masking agent.

Although the formation of metal fluoride complexes would be expected to be a problem in the IC procedures, Wilson and Gent (1982) found no evidence of it and did not add a masking agent. Because chloride elution closely follows fluoride in the IC method, if the chloride concentration greatly exceeds the fluoride concentration (as in evaporites), a fluoride determination might not be possible. In the analysis of phosphates by IC, the possibility of fluoride remaining in the insoluble residue from the sodium carbonate fusion can be avoided by digesting the residue in dilute sulfuric acid [R]. Sulfate elutes sufficiently long after fluoride that the elution peaks do not overlap severely.

In the analysis of coal for fluoride, which largely occurs as fluorapatite (Thomas and Gluskoter, 1974), the sample is ashed first. Magnesium oxide and magnesium nitrate are added during the ashing to retard the volatilization of fluoride. Because a sample size 10 times larger for coal than for rock samples is used, if aluminum in the coal exceeds 3-4 percent, the citrate buffer may not provide enough masking of aluminum fluoride complexes, and low results may be obtained by the ISE procedure (Ingram, 1970).

The majority of published papers describing ISE techniques for the determination of fluoride in geological materials report precision on the order of a 5- to 10percent relative standard deviation. A drift of $1 \mathrm{mV}$ in potential would represent a relative error of about 4 percent, which approximates the best precision that can be attained with an ISE procedure (Nicholson, 1983). In the Denver laboratory from 1979 to 1984 , the Geological Survey standard andesite AGV-1 was analyzed 196 times by the ISE procedure described above with a relative standard deviation of 15 percent. The mean value was 0.038 percent (on an as-received basis) with a range of 0.02-0.07 percent; Abbey's (1983) usable value was 0.04 percent F (on a dry-weight basis). In the period 1977 through 1980, National Bureau of Standards coal 1632 was analyzed on an as-received basis 152 times with a relative standard deviation of 9 percent. The mean value was 0.008 percent $F$ compared to four values in the literature ranging from 0.005 to 0.01 percent (Gladney and others 1984).

Wilson and Gent (1982) analyzed 18 geologic standards in triplicate by IC and found relative standard deviations of about 5 percent. Their limit of detection was approximately 0.0008 percent $(8 \mathrm{ppm}) \mathrm{F}$ in the sample $(0.1 \mathrm{~g})$. However, as for $\mathrm{Cl}$, the limit of determination is usually 0.0025 percent.

\section{Iron (II)}

\section{Summary of Method}

$[D, M, R] \quad$ The sample $(0.5 \mathrm{~g})$ is boiled with $\mathrm{HF}$ and $\mathrm{H}_{2} \mathrm{SO}_{4}$ in a platinum crucible. After about 10 min of boiling, the crucible is immersed in a solution of boric, sulfuric, and phosphoric acids. This solution is titrated with potassium dichromate using an automated potentiometric titrator with a platinum indicator electrode [D], an automated colorimetric titration [M], or a manual colorimetric titration [R]. Sodium diphenylamine sulfonate is used as the endpoint indicator in the colorimetric titration (Peck, 1964; Cremer and others, 1984).

\section{Reporting of Results}

Iron (II) is reported as percent $\mathrm{FeO}$ with a limit of determination of 0.01 percent. 


\section{Discussion}

Whereas the determination of iron (II) is one of the easiest and most important geochemical determinations, it is also one of those most prone to error and misinterpretation. In this determination, the analysis technique generally has less influence on the apparent speciation than does the sample matrix. Iron (II) minerals (which are resistant to acid digestion), species (which oxidize or reduce iron), and the titrant are commonly occurring

Hillebrand and others, (1953) discussed the oxidation of iron (II) during grinding. They suggested that, for silicates, generally little oxidation occurs with $15-30 \mathrm{~min}$ of grinding and that it depends on the mineralogy of a specific sample. They found up to 20- to 30-percent loss of $\mathrm{FeO}$ with $2 \mathrm{~h}$ of grinding. Numerous analysts recommend a coarse fraction (60 mesh) for the iron (II) determination; however, most laboratories, including ours, grind the sample to -100 mesh. Although finer grinding promotes aerial oxidation, it improves the digestibility of numerous minerals with mixed acids.

Many iron (II)-containing minerals are slow to dissolve or do not dissolve at all in hydrofluoric and sulfuric acids. Chromite, staurolite, tourmalines, amphiboles, axinites, pyroxenes, and spinels tend not to dissolve. Ilmenite and magnetite are dissolved incompletely, and garnets usually are dissolved only after repeated digestions. The carbonate siderite is quite acid resistant and is slow to dissolve (Peck, 1964). Prolonged boiling in hydrofluoric and sulfuric acids may dissolve more of these minerals; however, hot sulfuric acid promotes oxidation of iron (II) (Johnson and Maxwell, 1981).

Sulfide minerals pose additional problems. Pyrite alone is not attacked appreciably by hydrofluoric and sulfuric acids; however, if iron (III) is present in the sample, it promotes the dissolution of pyrite (Hillebrand and others, 1953). As the sulfur is oxidized during the dissolution, iron (III) is reduced, causing high results for iron (II). A similar problem occurs with acid-soluble sulfides, such as pyrrhotite. During decomposition, they release hydrogen sulfide, which reduces iron (III). A sample containing 0.1 percent $S$ could increase the iron (II) oxide concentration by up to 1.8 percent $\mathrm{FeO}-$

$$
\mathrm{H}_{2} \mathrm{~S}+4 \mathrm{Fe}_{2} \mathrm{O}_{3} \rightarrow \mathrm{SO}_{3}+8 \mathrm{FeO}+\mathrm{H}_{2} \mathrm{O} \text {. }
$$

Although one never knows to what extent reduction of iron (III) will occur, much of the hydrogen sulfide appears to be released by our digestion technique (Johnson and Maxwell, 1981). Thus, with either acid-soluble or insoluble sulfides present, the chemically determined iron (II) content is certainly questionable, if not grossly inaccurate.

Other species cause oxidation or reduction of iron problems. during the decomposition (Hillebrand and others, 1953;

Johnson and Maxwell, 1981). Manganese (IV), probably most commonly encountered as pyrolusite, and vanadium (V) will oxidize iron (II). Vanadium (III) will reduce iron (III). Although organic matter generally will not reduce iron (III), it will reduce the titrant, causing high results for iron (II). Graphite has no effect.

As a result of the numerous problems arising in the determination of the iron speciation in geologic materials, it has been proposed that "net state of oxidation" (Groves, 1951 ) or "oxygen excess or deficiency" (Ingamells, 1960) be reported in place of iron (II) oxide. Whatever the result reported, if a sample exhibits any of the problems discussed, it can be considered, at best, an estimate of the true concentration in the solid sample.

Despite the problems encountered, excellent precision can be attained for many sample types. Peck (1964) stated, "...replicate determinations should agree within 0.1 percent (absolute) and failure to achieve such agreement is caused either by mineral segregation or by incomplete solution of sample." Figure 2 shows examples of results obtained for two Geological Survey standards, andesite AGV-1 and Hawaiian basalt BHVO-1, in the Denver laboratory. The standard deviations were 0.04 and 0.01 percent absolute for BHVO-1 and AGV-1, respectively, with a relative standard deviation of 0.5 percent for both samples. The means of 8.58 and 2.05 for both samples agree well with Abbey's (1983) usable values of 8.55 and 2.03 for BHVO-1 and AGV-1, respectively. Thus, for samples that dissolve well and do not have any of the matrix problems discussed, reasonable precision and accuracy are easily attainable.

\section{Potassium and Sodium}

\section{Summary of Method}




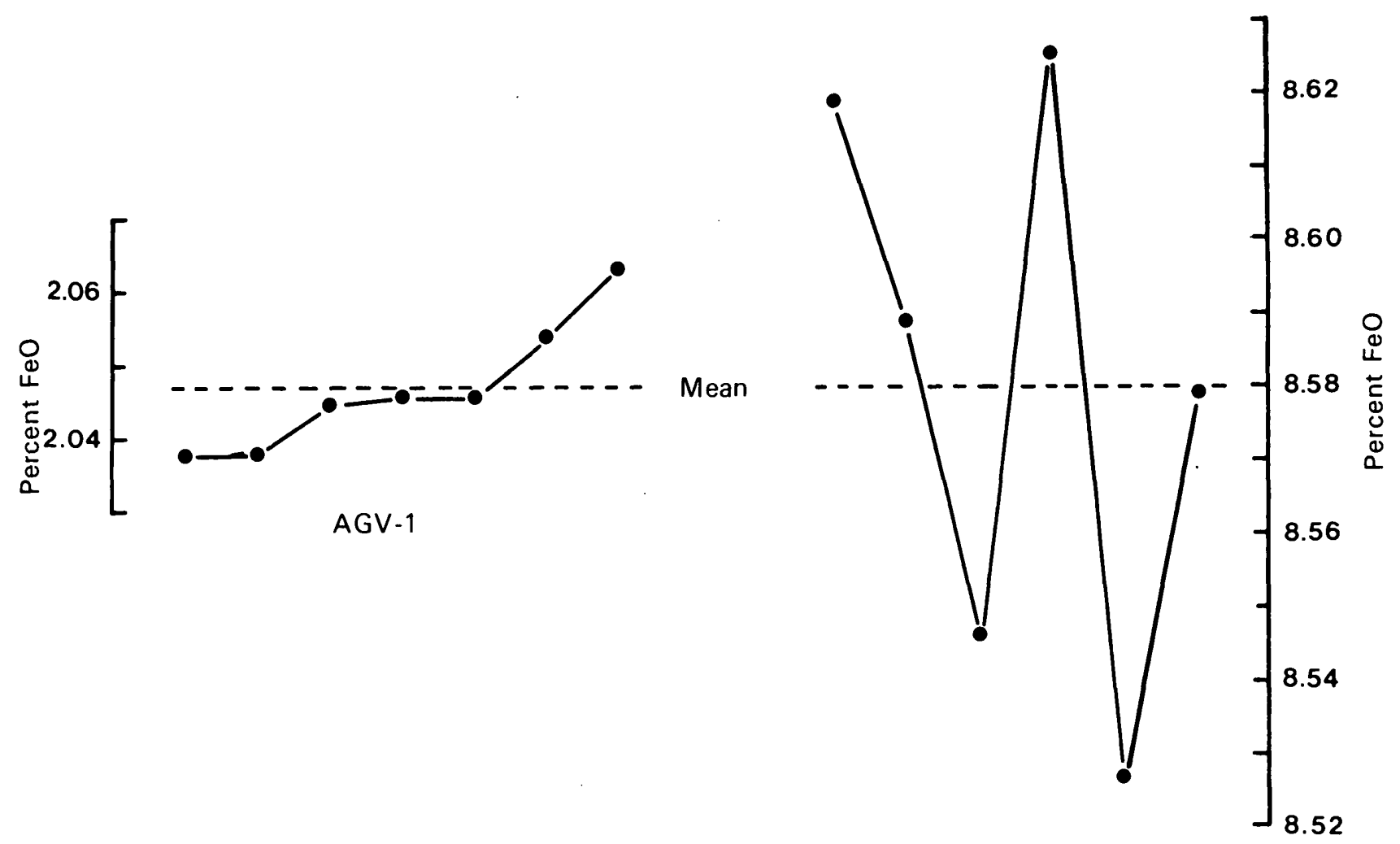

BHVO-1

\section{Chronological results}

Figure 2. Chronological results for the determination of iron (II) oxide in U.S. Geological Survey standards andesite AGV-1 and Hawaiian basalt BHVO-1 [AGV-1: mean $=2.05$ percent, standard deviation $=0.01$ percent; BHVO-1: mean $=8.58$ percent, standard deviation $=0.04$ percent (as-received basis); usable values (Abbey, 1983 ): $A G V-1=2.03$ percent, BHVO-1 $=8.55$ percent (dry-weight basis).

\section{Reporting of Results}

Sodium and potassium are reported as percent $\mathrm{Na}_{2} \mathrm{O}$ and percent $\mathrm{K}_{2} \mathrm{O}$ with a limit of determination of 0.01 percent for each.

\section{Discussion}

Flame emission spectrometry has replaced the tedious gravimetric procedures for the determination of the alkali metals. To overcome the variations in flame and sample aspiration parameters inherent in the flame technique, a fixed amount of lithium is added to samples and standards as an internal control. The lithium also serves as an ionization buffer so that the concentration determined for an individual alkali metal is not influenced by variations in the concentration of the other metal from sample to sample or sample to standard. Fixed, narrow bandpass, interference filters are used to isolate the emission lines for each element $-\mathrm{Na}, 589 \mathrm{~nm}$; Li, $671 \mathrm{~nm}$; and $\mathrm{K}, 766 \mathrm{~nm}$.

The samples are digested by fusion with lithium metaborate or by acid digestion with hydrofluoric and perchloric acids. Lithium metaborate is an excellent flux material that dissolves most minerals and produces a dilute, acid soluble melt (Johnson and Maxwell, 1981). A flux to sample ratio of 7 to 1 is used in the alkali metal determination. The acid digestion is not as widely applicable as the fusion technique if resistant minerals represent a major portion of the sample. However, samples with minor portions of resistant minerals, such as zircons or tourmalines, which do not dissolve in mixed acids, generally contain very little sodium or potassium, such that the small error produced through incomplete digestion is within the experimental error of the technique. Care must be taken in the acid digestion to remove the fluoride by fuming before introducing the sample into the flame because fluoride depresses the flame emission of potassium (Jeffery and Hutchison, 1981).

Calibration of the instrument is done using rock standards $[\mathrm{M}]$ or solution standards prepared from reagent-grade chemicals $[D, R]$. To minimize the influence of instrumental drift, sets of four to eight samples are bracketed by standards in the analysis sequence. Usually, each sample solution is analyzed several times, and the results are averaged. 
Rice (1976) performed an interlaboratory study of the potassium determination, in which the Menlo Park laboratory participated. He concluded, “...a betweenlaboratory variation in flame spectrometric potassium results of less than $0.5 \%$ relative standard deviation is attainable." No such study has been completed between the Geologic Division laboratories. Cremer and others, (1984) stated that precision of 0.1 percent absolute $\mathrm{K}_{2} \mathrm{O}$ is attainable. Data obtained in the Denver laboratory, which are presented in figure 3 , suggest that this may be true for samples analyzed within $1 \mathrm{~d}$ but not necessarily for a sample analyzed over a long period of time.

In Denver, the primary requests for sodium and potassium determinations are initiated by the geochronology laboratories. They submit samples as matched pairs with every eighth set a replicate pair of biotite from a quartz latitic ash-flow tuff. The control sample was analyzed 50 times (25 paired analyses) during 1984. The potassium results obtained are shown in figure 3 . The average range within each pair were 0.03 percent $\mathrm{K}_{2} \mathrm{O}$ and 0.005 percent $\mathrm{Na}_{2} \mathrm{O}$. The means were 8.76 percent $\mathrm{K}_{2} \mathrm{O}$ and 0.43 percent $\mathrm{Na}_{2} \mathrm{O}$ for all determinations with a pooled standard deviation of 0.03 percent absolute $\mathrm{K}_{2} \mathrm{O}$ and $<0.01$ percent absolute $\mathrm{Na}_{2} \mathrm{O}$. If the control sample submitted is truly homogeneous with respect to time, then the major portion of the analytical error in the alkali metal determination is attributable to differences between days. Interestingly, the accuracy and precision of the determination of potassium, a major element in rocks, is not as good as the determination of argon, which is present at $0.001-0.01 \mathrm{~mL}$ of gas per gram of sample.

The Menlo Park laboratory maintains a qualityassurance program for the determination of potassium. Three whole rock samples and six mineral separates are used in the program with two of the samples submitted as unknowns in each job. A summary of the results obtained from 1980 to 1985 is shown in table 4 .

\section{Sulfur}

\section{Summary of Methods}

\section{Total Sulfur}

[D,M,R] Total sulfur is determined by using a Leco SC-132 automated analyzer. Approximately $0.25 \mathrm{~g}$ of sample and $1 \mathrm{~g}$ of vanadium pentoxide flux are combusted at $1,370^{\circ} \mathrm{C}$ in an oxygen atmosphere (Scholz and Rathleff, 1982; Kirschenbaum, 1983; Jackson and others, 1985). An IR detector measures the sulfur dioxide evolved. Because the detector is nonlinear, the sample size is varied from 0.025 to $1 \mathrm{~g}$, depending on the sulfur content of the sample. Also, if the sample contains greater than 0.2 percent $F$ or greater than 1 percent

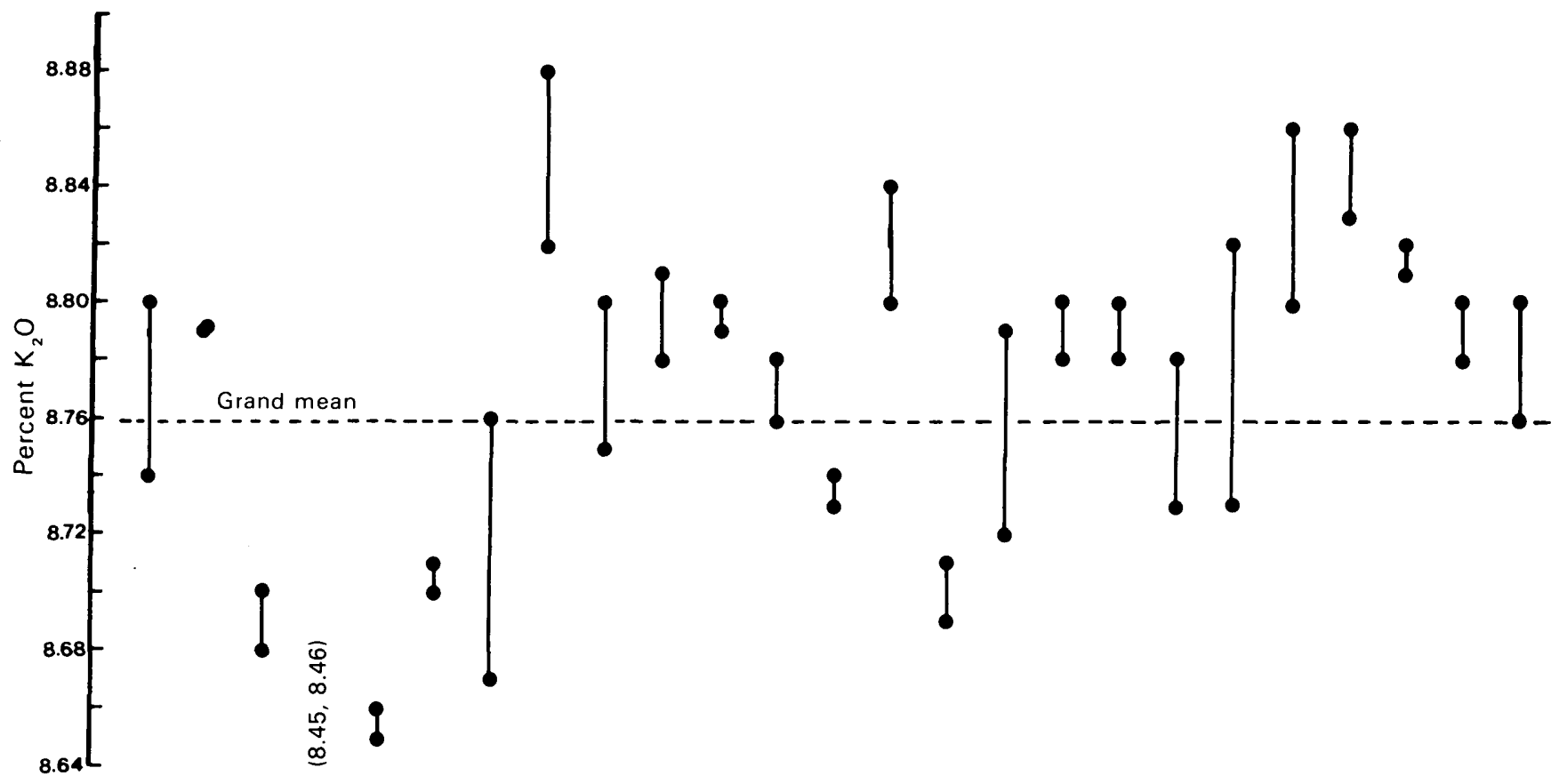

Chronological results

Figure 3. Paired chronological results between December 1983 and January 1985 for the determination of potassium in biotite control samples. 
Table 4. Summary of potassium determinations by flame emission spectrometry obtained in the Menlo Park laboratory quality-assurance program

[P. R. Klock, U.S. Geological Survey, written commun., 1985; RSD: Relative Standard Deviation]

\begin{tabular}{|c|c|c|c|c|}
\hline & Mean & & Standard & RSD \\
\hline Scale & percent $\mathrm{K}_{2} \mathrm{O}$ & $n$ & deviation & percent \\
\hline \multirow[t]{5}{*}{ Low } & 0.051 & 38 & 0.007 & 14 \\
\hline & 0.188 & 14 & 0.008 & 4.2 \\
\hline & 0.903 & 54 & 0.016 & 1.8 \\
\hline & 1.504 & 75 & 0.016 & 1.1 \\
\hline & 2.237 & 54 & 0.024 & 1.1 \\
\hline \multirow[t]{4}{*}{ High } & 2.95 & 35 & 0.03 & 1.2 \\
\hline & 5.53 & 37 & 0.07 & 1.2 \\
\hline & 10.63 & 27 & 0.04 & 0.4 \\
\hline & 13.62 & 41 & 0.07 & 0.5 \\
\hline
\end{tabular}

levels of molybdenum, then a reduced sample size or an alternate method is used. The sulfur may be determined alternately by using combustion in an induction furnace with a copper accelerator and an iodometric titration of evolved sulfur dioxide [D] (Johnson and Maxwell, 1981), or the sulfur is determined as sulfate ion by IC using an Eschka fusion $\left(\mathrm{MgO}-\mathrm{Na}_{2} \mathrm{CO}_{3}, 1: 2\right)$ for sample decomposition $[\mathrm{M}]$.

\section{Sulfate, Sulfide, and Organic Sulfur in Rocks}

Sulfur is determined on three separate splits of the sample by using a Leco SC-132 (as described above). The first split to be analyzed is untreated, thus total sulfur $\left(S_{T}\right)$ is determined. The second split $(\sim \mathrm{lg})$ is leached with $\mathrm{HCl}(1: 3)$, and the residue is analyzed for sulfur $\left(S_{R_{1}}\right)$. The third split $(\sim \lg )$ is leached with $\mathrm{HCl}$ (as above), and, then, the residue is leached further with $\mathrm{HNO}_{3}$ (1:7). The residue remaining after the $\mathrm{HCl}$ and $\mathrm{HNO}_{3}$ leaches is analyzed for sulfur $\left(S_{R_{2}}\right)$ and is a direct measure of organic sulfur. The sulfate and sulfide sulfur species are then determined by difference calculations (American Society for Testing and Materials, 1985; Johnson and Maxwell, 1981; Sobek and Bogner, 1984; Jackson and others, 1985).

$$
\begin{aligned}
S_{T}-S_{R 1} & =\text { Sulfate } S \\
S_{R 1}-S_{R 2} & =\text { Sulfide } S \\
S_{R 2} & =\text { Organic } S
\end{aligned}
$$

\section{Sulfate, Pyritic Sulfur, Acid-Volatile Sulfide, and Organic Sulfur in Coal}

A coal sample of $0.5 \mathrm{~g}$ ground to -325 mesh is leached with hydrochloric acid (1:3). Hydrogen sulfide, which evolved during the acid leach due to the dissolution of acidvolatile sulfides (AVS), is trapped in an alkaline zinc acetate solution. AVS sulfur is measured by determining total sulfur in the precipitate in the zinc acetate solution.

Sulfate sulfur is determined by analyzing for total sulfur in the barium sulfate precipitated from the hydrochloric acid leach. The residue from the hydrochloric acid leach is leached further with nitric acid (1:7). The acid leach solution is analyzed for total iron by atomic absorption spectroscopy. Pyritic sulfur is calculated stoichiometrically from the iron concentration. Organic sulfur is calculated by difference using the total sulfur concentration less the sulfide and sulfate sulfur (Karr, 1979; American Society for Testing and Materials, 1985).

\section{Reporting of Results}

Total sulfur, pyritic sulfur, AVS sulfur, sulfide sulfur, and organic sulfur are reported as percent $S$ with a limit of determination of 0.01 percent. Sulfate sulfur may be reported as percent $\mathrm{S}$, percent $\mathrm{SO}_{3}$, or percent $\mathrm{SO}_{4}$ with limits of determination of $0.01,0.03$, and 0.03 percent, respectively.

\section{Discussion}

The combustion-IR determination of total sulfur is suitable for the analysis of rocks, soils, coals, petroleum, and plant materials. In the combustion techniques using either IR detection or iodometric titration, the sulfur actually is determined as sulfur dioxide. Therefore, it is important that the sulfur in the sample is converted to sulfur dioxide and not to sulfur trioxide. Searle (1968) 
found that significant amounts of sulfur trioxide are formed when analyzing soils in an induction furnace with catalytic oxidants and iodometric titration of evolved sulfur. The extent to which this is a problem in the newer resistance furnace-IR instruments has not been determined. Nevertheless, if a constant ratio of $\mathrm{SO}_{2}$ to $\mathrm{SO}_{3}$ is obtained for the calibration standard and the sample, satisfactory results still should be obtained. However, matrix matching of samples and standards is frequently difficult.

Not only does the sulfur need to be evolved as sulfur dioxide, but the amount of sulfur flowing through the IR detector should be close to the amount during calibration. This is demonstrated by the analysis of SU-1, a Canadian sulfide ore, using two calibration standards and different sample sizes. As seen in table 5, the apparent sulfur content of SU-1 varies from 11.1 to 12.4 percent $S$ with varying analysis conditions. This points out, as in the determination of carbon, the need for standards that closely match the samples in the form and the concentra- tion of sulfur, as well as the sample matrix to minimize the effects of nonlinearity of the detector and differences between combustion characteristics of the standards and the samples.

The determination of specific forms of sulfur generally is restricted to rocks and coals with mixed success, depending upon the mineralogy of a sample. The forms of sulfur are divided into methodologically defined groupings dependent upon the chemical behavior of different species. Possible groupings are $\mathrm{HCl}$-soluble sulfates, $\mathrm{HCl}$ soluble sulfides, AVS, $\mathrm{HCl}$-insoluble sulfates, $\mathrm{HNO}_{3}$ soluble sulfides, pyritic sulfur, elemental sulfur, and organic sulfur. Thus, an understanding of the analysis procedures and of the mineralogy of a sample is required to interpret results reported as simply sulfate or sulfide sulfur.

The determination of forms of sulfur in rocks relies on the selective leaching of sulfur species by hydrochloric and nitric acids. As defined by methodology used in our laboratories, sulfate sulfur is $\mathrm{HCl}$-soluble sulfate that

Table 5. Sample size and calibration dependency of the apparent sulfur content in sulfide ore SU-1

[L. L. Jackson and E. E. Engleman, U.S. Geological Survey, written commun., 1985; RSD: Relative Standard Deviation]

\begin{tabular}{|c|c|c|c|c|c|}
\hline $\begin{array}{l}\text { Calibrating } \\
\text { standard }\end{array}$ & $\begin{array}{l}\text { Sample } \\
\text { size (mg) }\end{array}$ & $\begin{array}{c}\text { Mominal } \\
\text { mg of } \\
s^{1}\end{array}$ & $\frac{\text { mgS sample }}{\text { mgs std. }}$ & $\begin{array}{l}\text { Mean }(n=3) \\
\text { apparent } \\
5 \\
\text { percent }\end{array}$ & $\begin{array}{c}\text { RSD } \\
\text { percent }\end{array}$ \\
\hline $\begin{array}{l}\text { National Bureau } \\
\text { of Standards }\end{array}$ & & & & & \\
\hline 2685 coal SPY & 300 & 14 & -- & 4.62 & $\cdots$ \\
\hline \multirow[t]{3}{*}{ SU-1 } & 165 & 20 & 1.4 & 11.5 & 0.8 \\
\hline & 116 & 14 & 1 & 11.2 & 2.8 \\
\hline & 58 & 7 & 0.5 & 11.1 & 0.5 \\
\hline
\end{tabular}

National Bureau

of Standards

113 a zinc ore

60

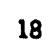

$--$

30.6

concentrate SRM

su-1

153
113
75
37

18

1

12.4

0.9

0.75

12.4

1.3

0.5

11.8

0.5

37

4

0.25

11.2

2.9

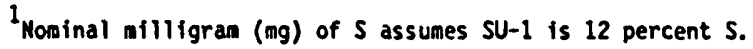


excludes sulfate-containing minerals, such as barite and scapolite, which are insoluble in hydrochloric acid, and alunite and celestite, which are only partially attacked (Peck, 1964; Johnson and Mazwell, 1981). Some sulfides (AVS), such as pyrrhotite, are soluble in hydrochloric acid. Although these sulfides decompose, giving off hydrogen sulfide gas, iron (III) in solution promotes the oxidation of $\mathrm{HCl}$-soluble sulfides to sulfate. Therefore, some AVS may be included in the sulfate sulfur, if sulfate is determined directly in the leach solution, unless precautions are taken (Peck, 1964). In the difference procedure currently used in Denver, all AVS would be included as sulfate sulfur.

Sulfide sulfur is defined methodologically $[D]$ in rock analysis as soluble sulfur after removal of $\mathrm{HCl}$ soluble sulfur. Pyrite and marcasite are the primary examples. AVS would not be included as sulfide sulfur. In the coal analysis procedure [R], the total iron content of the nitric acid leach is determined, and pyritic sulfur is reported based on stoichiometric calculations.

In rock analysis, especially in highly mineralized samples, much of the $\mathrm{HNO}_{3}$-soluble sulfur may come from sulfide minerals other than pyrite. Thus, the determination of iron in the leach may misrepresent totally the sulfide sulfur content of a sample. Also, any iron (III) minerals soluble in nitric acid, but not removed in the hydrochloric acid leach, would contribute to the apparent pyritic sulfur content. Evidence has been found that in coal samples microcrystalline pyrite is encapsulated in organic matter or clay aggregates and does not dissolve entirely in dilute nitric acid (Stanton and Renton, 1981). This may occur in rocks rich in organic material as well. Also some easily oxidizable organic sulfur species and elemental sulfur may dissolve in the nitric acid leach contributing to the sulfide sulfur content. Alunite and celestite also are attacked by nitric acid; therefore, the sulfur in them would be included in the sulfide sulfur.

In the determination of pyritic sulfur in coals, many of the problems encountered in rock analysis commonly are not found. However, it is not clear to what extent solubilization of other iron minerals and incomplete dissolution of pyrite due to encapsulation may be a problem. Grinding to -325 mesh may ensure dissolution of pyrite and help eliminate inhomogeneity due to density differences, but pyrite oxidation during grinding may be promoted.

Organic sulfur in rocks is determined directly by measuring the total sulfur content of the hydrochloric and nitric acid leached residue, whereas, in coal analysis, it is calculated by difference after the other forms of sulfur are determined. Both procedures suffer from some common problems - the organic sulfur content would include acidinsoluble sulfates, such as barite, elemental sulfur, and undissolved encapsulated pyrite. The difference method also would include any AVS that were lost as hydrogen sulfide and any errors made in the sulfate and sulfide species. The direct method would exclude any acidsoluble organic species.

A comparison of several speciation procedures was made using Geological Survey rock standards, green river shale SGR-1 and marine sediment MAG-1. In the Denver laboratory before 1986 , the determination of sulfate and sulfide sulfur in rocks was based on the direct measurement of sulfur in barium sulfate precipitates obtained from the hydrochloric and nitric acid leach solutions (one sample split was leached sequentially as in the coal procedure; however, $\mathrm{BaSO}_{4}$ was precipitated from $\mathrm{HNO}_{3}$ leach solution instead of determining iron in the leach). This speciation procedure and the difference method described above for rock analysis were used in the comparison. Also, total iron was determined in the nitric acid leach solution to calculate stoichiometrically pyritic sulfur as in the coal analysis procedure. The results are summarized in table 6.

The mean total sulfur contents for SGR-1 and MAG-1 were $1.45 \pm 0.02$ and $0.34 \pm 0.01$ percent $S \pm 1 \sigma$, respectively, whereas Abbey's (1983) usable values were 1.56 ? and 0.43 ? percent $S$. The reason for the discrepancy in total sulfur is not clear; however, the speciation results in table 6 should be internally consistent. It is difficult to compare the procedures with the limited data presented; however, some general observations can be made. The difference measurements of the sulfur species tend to give higher results for the individual sulfur species than do the direct procedures. Based on the amount of sulfur present and the mineralogy of the two samples, the difference measurements for the sulfate and sulfide species are probably more accurate than the direct results, whereas the direct determination of organic sulfur is probably more accurate than the result by difference.

The two samples appear to have an insignificant amount of AVS ( $\leq 0.01$ percent $S$ ); therefore, the sulfate direct or the difference measurements should represent only sulfate for MAG-1, whereas the difference procedure obtained higher sulfate for SGR-1 than did the direct procedure. This is not unreasonable if one considers the difficulties in obtaining complete recovery of the $\mathrm{BaSO}_{4}$ precipitate. We believe the sulfate content determined by difference is more representative of the true value for these samples.

In comparing the direct and the difference procedures for the determination of sulfide sulfur, the difference procedure yielded higher results, as it did for sulfate sulfur. This may be due to incomplete recovery of the sulfide sulfur as $\mathrm{BaSO}_{4}$. The estimation of sulfide sulfur based on the determination of iron in the nitric acid leach solution yielded even higher results than the other two procedures. Because the summation of sulfate and organic sulfur determined directly and the sulfide sulfur calculated stoichiometrically from the iron content yields more 
Table 6. Forms of sulfur by different speciation techniques (dry-weight basis, $n=3$ ) [In percent sulfur. Jackson and others (1985b)]

\begin{tabular}{|c|c|c|c|c|c|c|c|c|}
\hline & \multirow{2}{*}{$\frac{\text { Bulk Sample }}{\text { Total S }}$} & \multicolumn{2}{|c|}{ Sulfate $S$} & \multicolumn{3}{|c|}{ Sulfide $S$} & \multicolumn{2}{|c|}{ Organic $S$} \\
\hline & & Direct $^{1}$ & Difference $^{2}$ & Direct $^{1}$ & Difference ${ }^{2}$ & ${ }^{3} \mathrm{AS} \mathrm{FeS}_{2}$ & Difference ${ }^{1.4}$ & Direct $^{2,5}$ \\
\hline SGR-1, ofl shale & 1.45 & 0.06 & 0.20 & 0.85 & 0.91 & 1.22 & 0.49 & 0.35 \\
\hline KAG-1, marine sediment & 0.34 & 0.18 & 0.17 & 0.06 & 0.13 & 0.18 & 0.10 & 0.04 \\
\hline \multicolumn{9}{|c|}{ 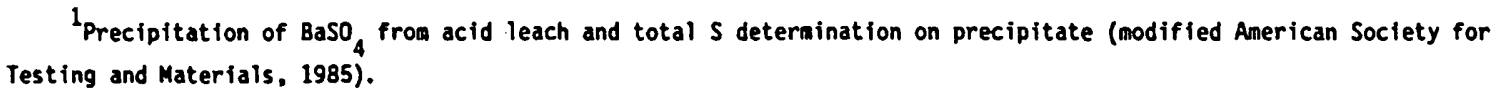 } \\
\hline \multicolumn{9}{|c|}{$\begin{array}{l}{ }^{2} \text { Total } S \text { deternination on acid-leached residues and calculation of sulfur species by difference (Jackson and } \\
\text { others, 1985b). }\end{array}$} \\
\hline \multicolumn{9}{|c|}{$\begin{array}{l}{ }^{3} \mathrm{Fe} \text { deternination in } \mathrm{HNO}_{3} \text { leach and stoichiometric calculation as pyrite (American Society for Testing and } \\
\text { Materials, 1985). }\end{array}$} \\
\hline $\begin{array}{l}\text { 4Organic S calcul } \\
\text { and Materials, 1985). }\end{array}$ & lated by dif & ference us & sing total $S$ a & didect S & $S$ species measi & Jrements (A & erican Society $f$ & for Testing \\
\hline
\end{tabular}

total sulfur than was determined directly in the bulk sample, it appears that iron species other than pyrite are being dissolved by the nitric acid leach for these samples. Therefore, the iron determination is not performed for samples other than coal.

Although the recovery of sulfate or sulfide sulfur is difficult to determine unless the mineralogy of a sample is well characterized, an estimate for sulfate recovery in MAG-1 can be made by assuming that the sulfate sulfur present is due to the evaporation of seawater during preparation of the standard. In this instance, the $\mathrm{Cl}$ to $\mathrm{S}$ ratio would be about 18.7:0.9 (Manheim and others, 1976; Krauskopf, 1979) based on the ratio in seawater. Using Abbey's (1983) value for chloride (3.09? percent) in MAG-1, one would expect 0.15 percent sulfate sulfur, and we found 0.17 percent, indicating good recovery of sulfate species by our procedures. However, Manheim and others (1976) only found 0.05 percent sulfate sulfur $\left(0.15\right.$ percent $\left.\mathrm{SO}_{3}\right)$. This discrepancy in sulfate content may be due to differences in the analytical procedures used or it may be a result of pyrite oxidation in the standard since its preparation.

Manheim and others (1976) found about 1 percent pyrite $(\mathrm{S}=0.53$ percent $)$ in MAG-1 by $\mathrm{X}$-ray diffraction.
They also determined the total $S$ to be 0.52 percent. Assuming that most of this sulfur is pyritic sulfur, their ratio of sulfide to sulfate (about $9.4: 1$ ) is considerably larger than what we found (1.3:1), indicating that pyrite oxidation probably has occurred to a significant extent in the sample. However, pyrite oxidation does not explain entirely the discrepancies in sulfur results, and it is suspected that most of the differences are due to the differences in the analytical procedures.

The determination of organic sulfur by either the direct or the difference procedure is not entirely satisfactory. Elemental sulfur, barite, and encapsulated pyrite may remain in the hydrochloric and nitric acid leached residue and, thus, be included as organic sulfur by either procedure. The difference procedure has the added disadvantage of including the AVS and accumulating the analytical error associated with the determination of the other forms of sulfur. For SGR-1 and MAG-1, the difference procedure yielded higher results than the direct procedure. The direct determination would appear to give the better estimate of organic sulfur. This can be further examined by comparing the ratios of organic $\mathrm{C}$ to organic $S$ obtained by the two procedures (see table 7). Kaplan and others (1963) found organic $C$ to organic $S$ from 75:1 
Table 7. Organic carbon-organic sulfur ratios in green river shale SGR-1 and marine sediment MAG-1

[L. L. Jackson and E. E. Engleman, U.S. Geological Survey, written commun., 1985]

\begin{tabular}{lccccc}
\hline & \multicolumn{3}{c}{ Organic S } & \multicolumn{2}{c}{ Organic C/Organic S } \\
Standard & Organic C & Difference & Direct & Difference & Direct \\
& & & & & \\
\hline SGR-1, ofl shale & 24.2 & 0.49 & 0.35 & 49 & 69 \\
MAG-1, marine sediment & 2.2 & 0.10 & 0.04 & 22 & 55 \\
\hline
\end{tabular}

to 550:1 in California marine sediments. When compared to the results of Kaplan and others (1963), the higher ratios of organic $\mathrm{C}$ to organic $\mathrm{S}$ obtained using the direct determination of organic $S$ appear more reasonable than those obtained using results from the difference procedure.

As discussed above, the accuracy of the sulfur speciation procedures is very dependent upon the mineralogy of a specific sample. Fortunately, the precision of these procedures is reasonably good. When the sulfur content, as either sulfide or sulfate, exceeded 0.1 percent, the relative standard deviation was 5-10 percent. The relative error increased greatly (25-50 percent) at the lower concentrations. In general, if the total sulfur is 0.1 percent or less, then the Denver laboratory does not perform the sulfur speciation procedures. Peck (1964) stated, "...if small amounts of sulfur are present, it is best to determine and report total sulfur and to allow the petrographer to estimate its mineralogical distribution."

\section{Water}

Summary of Methods

\section{Moisture $\left(\mathrm{H}_{2} \mathrm{O}-\right)$}

$[D, R] \quad$ Moisture, or nonessential water $\left(\mathrm{H}_{2} \mathrm{O}-\right)$, is determined by weight loss of a 1-g sample after heating for a minimum of $1 \mathrm{hr}$ at $110^{\circ} \mathrm{C}$.

$[D, M] \quad$ It also is determined by heating $50 \mathrm{mg}$ of sample at $110^{\circ} \mathrm{C}$ and by coulometrically measuring the evolved water in a Karl Fischer titration (Norton, 1982).
Total Water and "Essential" or "Bound" Water $\left(\mathrm{H}_{2} \mathrm{O}+\right)$

[D,M] $\quad \mathrm{H}_{2} \mathrm{O}+$ is determined by difference calculation using the total water and $\mathrm{H}_{2} \mathrm{O}$ - water concentrations. The total water content is determined by heating $50 \mathrm{mg}$ of sample with $150 \mathrm{mg}$ of lead oxide and lead chromate flux at $900-950^{\circ} \mathrm{C}$. The evolved water is determined coulometrically with a Karl Fischer titration. $\mathrm{H}_{2} \mathrm{O}+$ may be determined directly in the same fashion using a moisture-free sample (Johnson and Maxwell, 1981; Norton, 1982).

[R] $\quad \mathrm{H}_{2} \mathrm{O}+$ also is determined directly using an automated $\mathrm{C}, \mathrm{H}, \mathrm{N}$ analyzer. Dried samples $\left(15-20 \mathrm{mg}\right.$ at $110^{\circ} \mathrm{C}$ overnight) are analyzed at $950^{\circ} \mathrm{C}$ (Din and Jones, 1981; Skinner and others, 1981).

\section{Reporting of Results}

Total $\mathrm{H}_{2} \mathrm{O}, \mathrm{H}_{2} \mathrm{O}+$, and $\mathrm{H}_{2} \mathrm{O}$ - are reported as weight-percent $\mathrm{H}_{2} \mathrm{O}$ with a limit of determination of 0.01 percent $\mathrm{H}_{2} \mathrm{O}$.

\section{Discussion}

Analytically, water in a geologic material is divided into two fractions - moisture (nonessential or $\mathrm{H}_{2} \mathrm{O}-$ ) and essential (bound or $\mathrm{H}_{2} \mathrm{O}+$ ). $\mathrm{H}_{2} \mathrm{O}$ - is defined as water lost from a sample at $100-110^{\circ} \mathrm{C}$, and $\mathrm{H}_{2} \mathrm{O}+$ is all remaining water, which usually is determined by evolution at $900-1,000^{\circ} \mathrm{C}$ with the aid of a flux. Although these definitions are somewhat arbitrary, $\mathrm{H}_{2} \mathrm{O}$ - is suitable for correcting most samples to a dry-weight basis, and $\mathrm{H}_{2} \mathrm{O}+$ is suitable for most mineralogical or petrographic purposes. Loss on ignition at $1,000^{\circ} \mathrm{C}$ usually is not a suitable 
estimation of the water content of a sample. For rigorous interpretation of the water content of a sample, a study by evolved gas analysis, thermogravimetric analysis, and so forth of water loss versus temperature usually is required.

Hydrogen may occur in minerals as hydrogen, hydroxyl groups, and (or) water molecules. The form of hydrogen and the nature of its interaction with the crystal structure determine its classification. Hillebrand and others (1953) defined nonessential hydrogen as "...its presence not necessary for the characterization of a mineral" and essential hydrogen as "...present in regular atomic arrangement in molecular or crystal structure." It is clear that no sharp boundary exists between nonessential and essential hydrogen or $\mathrm{H}_{2} \mathrm{O} \pm$. Of the free mineral water species, hygroscopic or capillary water and colloidal water usually are removed by heating to $110^{\circ} \mathrm{C}$, and zeolitic water is removed by heating from 80 to $400^{\circ} \mathrm{C}$ continuously. In some minerals, water of crystallization (hydrated water) may be removed from room temperature up to several hundred degrees (Todor, 1976). Hydroxyl ions generally are evolved after water of crystallization and at much higher temperatures, in some cases requiring over $1,000^{\circ} \mathrm{C}$.

Amphiboles, epidotes, kaolin group, talc, staurolite, topaz, chondrodite, titanite, phlogopite, and biotite are examples of minerals that do not give up their water easily (Groves, 1951). Usually, high temperature $\left(900-1,000^{\circ} \mathrm{C}\right)$ and a flux are required for complete release of water. The flux not only helps break down the mineral structure, but retains other volatile elements such as sulfur, fluorine, and chlorine that may interfere in the water determination (Peck, 1964). The flux also prevents the reduction of water to hydrogen by iron (II).

The presence of organic matter in the sample certainly affects the accuracy of the $\mathrm{H}_{2} \mathrm{O}$ - and the $\mathrm{H}_{2} \mathrm{O}+$ determinations if it does not preclude totally their determination. The loss of volatile organic species at $110^{\circ} \mathrm{C}$ yields high results in the gravimetric $\mathrm{H}_{2} \mathrm{O}$ - determination. High results also are obtained for total water or $\mathrm{H}_{2} \mathrm{O}+$ content of samples due to combustion of the organic matter yielding water and carbon dioxide.

Sample preparation or grinding also may affect the $\mathrm{H}_{2} \mathrm{O} \pm$ content of samples. The increase in surface area may cause an increase in adsorbed moisture. Heat generated during grinding may cause volatilization of adsorbed or zeolitic water and even of some water of crystallization. Also, fluid inclusions may rupture. Clays and other hygroscopic species are particularly sensitive to atmospheric conditions with the possibility of a change in moisture content of several percentage points over a short time period.

The analysis of the micas shown in table 8 clearly demonstrates the need for a flux if all the water is to be removed from certain minerals. Peck (1964) tested the reactivity of several fluxes by analyzing two amphiboles,
Table 8. Direct determination of watert in micamagnesium and mica-iron standards by Karl Fischer titration [Jackson and others, 1985c; RSD: relative standard deviation]

$$
\begin{aligned}
& \mathrm{H}_{2} \mathrm{O}+ \\
& \text { percent }
\end{aligned}
$$

With flux

Without flux (RSD, $n=4$ ) Usabie value ${ }^{1}$

$\begin{array}{llll}\text { Mica-Mg, phlogopite } & 1.08 & 2.24(2.0) & 2.10 ? \\ \text { Mica-Fe, biotite } & 2.38 & 2.96(1.7) & 2.91\end{array}$

$1_{\text {Abbey (1983). }}$

hornblende, and uralite and found the lead oxide and lead chromate flux, which is used in the Penfield water method and now used on all samples in the Karl Fischer titration method, to be the most satisfactory. Skinner and others (1981) found that most rocks, with the obvious exception of those containing micas, released their $\mathrm{H}_{2} \mathrm{O}+$ without a flux. They used vanadium pentoxide with a sample-to-flux ratio of 3 to 1 for samples high in mica analyzed by an automated $\mathrm{C}, \mathrm{H}, \mathrm{N}$ analyzer, which is the procedure currently used in the Reston laboratory.

Skinner and others (1981) determined the $\mathrm{H}_{2} \mathrm{O}+$ content of 30 international geochemical standards using an automated $\mathrm{C}, \mathrm{H}, \mathrm{N}$ elemental analyzer. They concluded that the small sample size $(20 \mathrm{mg})$ did not cause a severe sampling problem. They obtained relative standard deviations of about 1-7 percent for samples with 0.1-12 percent $\mathrm{H}_{2} \mathrm{O}+$. Norton (1982) analyzed 27 Geological Survey rock standards for $\mathrm{H}_{2} \mathrm{O}+$ using the Karl Fischer titration. $\mathrm{He}$ obtained standard deviations for the $\mathrm{H}_{2} \mathrm{O}+$ determinations in the range of $0.01-0.08$ percent absolute for samples with less than 1 percent $\mathrm{H}_{2} \mathrm{O}+$ or $\mathrm{H}_{2} \mathrm{O}-$. Jackson and others (1985c) also analyzed a variety of international rock standards, including the two micas shown in table 7 ranging in $\mathrm{H}_{2} \mathrm{O}+$ content from 0.1 to 11 percent with relative standard deviations of from 2 to 4 percent. Based on the work of Norton (1982) and Jackson and others (1985c), no bias was found between the results obtained by Karl Fischer titration and Abbey's (1983) usable values, except for samples containing less than 0.5 percent $\mathrm{H}_{2} \mathrm{O}+$ where the Karl Fischer titration gave low results by $0.05-0.1$ percent absolute.

The elemental analyzer $(\mathrm{C}, \mathrm{H}, \mathrm{N})[\mathrm{R}]$, the Karl Fischer coulometric titration [D], and a coulometric pro- 
cedure described by Cremer and Elsheimer (1972) [M] have been used for the determination of water in small mineral samples. In the microcoulometric procedure of Cremer and Elsheimer, water is released by combustion with a $\mathrm{PbO}-\mathrm{PbCrO}_{4}-\mathrm{CuO}$ flux and absorbed by a phosphorus pentoxide film where it is then electrolyzed to hydrogen and oxygen. The number of coulombs used in the electrolysis is proportional to the water released. They analyzed several minerals, including several micas, an amphibole, and an epidote, ranging in total water content from about 0.5 to 10 percent. They used sample sizes from 20 to $70 \mathrm{mg}$ and obtained relative standard deviations of from 2 to 4 percent.

Jackson and others (1985c) analyzed by Karl Fischer titration a variety of hand-picked specimens of phosphate, carbonate, and sulfate minerals ranging in total water content from about 10 to 35 percent. They used 5-mg sample sizes and obtained relative standard deviations of $0.2-1.5$ percent. Their results also compared well with the theoretical water content of the minerals.

\section{Classical Whole Rock Analysis}

\section{Summary of Methods}

$$
\mathrm{H}_{2} \mathrm{O}-, \mathrm{SiO}_{2}, \mathrm{Al}_{2} \mathrm{O}_{3}, \mathrm{TiO}_{2}, \mathrm{Fe}_{2} \mathrm{O}_{3}, \mathrm{MgO} \text {, and } \mathrm{CaO}
$$

A 1-g sample is dried in an oven at $105^{\circ} \mathrm{C}$ for $1 \mathrm{~h}$. $\mathrm{H}_{2} \mathrm{O}$ - is determined by weight loss. The sample is fused with $\mathrm{Na}_{2} \mathrm{CO}_{3}$. Silica is removed by precipitation with $\mathrm{HCl}$. $\mathrm{R}_{2} \mathrm{O}_{3}$ precipitation separates $\mathrm{Al}, \mathrm{Fe}$, and $\mathrm{Ti}$ from $\mathrm{Ca}$ and $\mathrm{Mg}$. The $\mathrm{TiO}_{2}$ and $\mathrm{Fe}_{2} \mathrm{O}_{3}$ residue is fused with potassium pyrosulfate. The titanium is determined colorimetrically with $\mathrm{H}_{2} \mathrm{O}_{2}$. For the determination of total iron, the Ti solution is passed through an $\mathrm{Ag}$ reductor, and the $\mathrm{Fe}$ is titrated with $\mathrm{K}_{2} \mathrm{Cr}_{2} \mathrm{O}_{7} . \mathrm{Al}_{2} \mathrm{O}_{3}$ and $\mathrm{Fe}_{2} \mathrm{O}_{3}$ are calculated by difference. $\mathrm{Mn}$ is removed from the $\mathrm{Ca}$ and $\mathrm{Mg}$ containing solution. The $\mathrm{Ca}$ and $\mathrm{Mg}$ are determined gravimetrically by precipitation as the oxalate and ammonium phosphate, respectively.

\section{$\mathrm{MnO}$ and $\mathrm{P}_{2} \mathrm{O}_{5}$}

A 1-g sample is ignited in a platinum dish. The sample is treated twice with $\mathrm{HF}$ and $\mathrm{HNO}_{3}$ and is evaporated to dryness. The residue is dissolved in $\mathrm{HNO}_{3}$ and boric acid and diluted to volume. The sample solution is split to determine $\mathrm{MnO}$ and $\mathrm{P}_{2} \mathrm{O}_{5}$ separately. Manganese is oxidized to permanganate with periodic acid, and the Mn content is determined colorimetrically. Phosphorus is precipitated as ammonium phosphomolybdate, redissolved, and determined colorimetrically as yellow vanadium molybdophosphate complex.

\section{$\mathrm{FeO}, \mathrm{Cl}, \mathrm{F}, \mathrm{Na}_{2} \mathrm{O}, \mathrm{K}_{2} \mathrm{O}, \mathrm{S}, \mathrm{CO}_{2}$, and $\mathrm{H}_{2} \mathrm{O}+$}

These determinations were outlined more thoroughly in the previous sections. $\mathrm{FeO}$ is determined by titration with $\mathrm{K}_{2} \mathrm{Cr}_{2} \mathrm{O}_{7}$ after acid digestion of the sample. $\mathrm{Cl}$ and $\mathrm{F}$ are determined using ISE. $\mathrm{Na}_{2} \mathrm{O}$ and $\mathrm{K}_{2} \mathrm{O}$ are determined by flame photometry. $\mathrm{S}$ is determined by using a Leco automated combustion analyzer. Total water is determined by the Penfield method (Peck, 1964). $\mathrm{H}_{2} \mathrm{O}+$ is calculated by difference. $\mathrm{CO}_{2}$ is determined coulometrically.

\section{Reporting of Results}

The results for classical whole rock analysis are reported in percentages as the oxide. The limit of determination is 0.01 percent for all constituents.

\section{Discussion}

Peck (1964) described and discussed the techniques used for whole rock analysis in the Denver laboratory. Many of the techniques were adapted from Washington (1904) and Hillebrand (1919); however, he described the use of special equipment that improved the convenience and ultimately the reliability of analyses when performed on a daily basis. More recently, Kirschenbaum (1983) described the methodology used in the Reston laboratory. His manual briefly described several of the newer instrumental procedures, such as the use of flame photometry and the combustion techniques. The majority of the manual is composed of step-by-step method descriptions adapted from Peck (1964) and Maxwell (1968). Cremer and others (1984) have described many of the step-bystep procedures used in the Menlo Park laboratory.

Today, relatively few classical "whole" rock analyses are done. X-ray fluorescence (XRF) spectroscopy has replaced, for the most part, the classical techniques. The speed and stability offered by modern spectrometers usually outweigh the few advantages offered by the experienced classical analyst. Although the classical techniques generally are more precise than the XRF analysis and the results obtained by an experienced analyst appear to be subject to less drift with time than those obtained by the XRF technique, the need for large numbers of analyses quickly is generally more pressing.

Table 9 gives several examples of the precision of the classical techniques. Flanagan and Kirschenbaum (1984) analyzed three Geological Survey standards, basalt BIR-1, diabase DNC-1, and diabase W-2, five times each and calculated "error standard deviations for the procedures." Jackson and others (1984) analyzed Hawaiian basalt BHVO-1 three times each by two experienced analysts and determined pooled standard deviations. Also included in table 9 are the limits of error for 
Table 9. Examples of the precision for the classical whole rock analysis

I. Error standard deviations, the square root of the error mean square in the analysis of variance, $n=15$ (Flanagan and Kirschenbaum, 1984).

II. Pooled standard deviations for Hawaiian basalt BHVO-1, two analysts, three analyses each (Jackson and others, 1984).

III. Expected error for first-class work (Groves, 1951).

[--not determined (Jackson and others) or not specified (Groves, 1951)]

\begin{tabular}{|c|c|c|c|}
\hline Oxide & I & II & III \\
\hline $\mathrm{SiO}_{2}$ & 0.070 & 0.06 & 0.20 \\
\hline $\mathrm{Al}_{2} \mathrm{O}_{3}$ & 0.15 & 0.10 & 0.10 \\
\hline $\mathrm{Fe}_{2} \mathrm{O}_{3}$ & 0.059 & 0.02 & 0.03 \\
\hline FeO & 0.046 & 0.02 & 0.02 \\
\hline MgO & 0.032 & 0.02 & 0.03 \\
\hline $\mathrm{CaO}$ & 0.020 & 0.02 & 0.02 \\
\hline $\mathrm{Na}_{2} \mathrm{O}$ & 0.012 & 0.02 & 0.03 \\
\hline$k_{2} O$ & 0.0039 & 0.09 & 0.02 \\
\hline $\mathrm{H}_{2} \mathrm{O+}$ & 0.041 & 0.02 & 0.02 \\
\hline $\mathrm{H}_{2} \mathrm{O}-$ & 0.0082 & $\cdots$ & $\cdots$ \\
\hline $\mathrm{THO}_{2}$ & 0.0082 & 0.04 & 0.01 \\
\hline$P_{2} O_{5}$ & 0.0091 & $<0.01$ & 0.01 \\
\hline MnO & 0.0022 & $<0.01$ & 0.01 \\
\hline $\mathrm{CO}_{2}$ & 0.005 & $<0.01$ & $\cdots$ \\
\hline
\end{tabular}

first-class work, as defined by Groves (1951). These three examples are typical for the analysis of silicates; however, results may vary greatly for different types of samples.

Accuracy cannot be truly assessed; however, summation of the constituents is used frequently as an indicator. Peck (1964) stated that a summation between 99.50 and 100.25 is acceptable, but Hillebrand (1919) considered 99.75-100.50 acceptable. For more discussion of the errors inherent in the classical techniques, one should refer to Groves (1951), Hillebrand and others (1953),
Peck (1964), and Johnson and Maxwell (1981). Chalmers and Page (1975) discussed the reporting of results for analyses of silicate rocks. They made a case that no more than three significant figures can be justified in the reporting of major-element determinations. Up to four significant figures commonly are reported for major oxides by our laboratory.

\section{Rapid Rock Major-Element Determination}

\section{Summary of Methods \\ $\mathrm{SiO}_{2}, \mathrm{Al}_{2} \mathrm{O}_{3}, \mathrm{Fe}_{2} \mathrm{O}_{3}, \mathrm{MgO}, \mathrm{CaO}, \mathrm{Na}_{2} \mathrm{O}, \mathrm{K}_{2} \mathrm{O}, \mathrm{TiO}_{2}, \mathrm{P}_{2} \mathrm{O}_{5}$ and $\mathrm{MnO}$}

The sample $(0.1 \mathrm{~g})$ is fused with $0.6 \mathrm{~g} \mathrm{LiBO}_{2}-$ $\mathrm{Li}_{2} \mathrm{~B}_{4} \mathrm{O}_{7}$ flux (1:2) in graphite crucibles for $45 \mathrm{~min}$ at $1,000^{\circ} \mathrm{C}$. The fused sample is dissolved in dilute nitric acid and analyzed by inductively coupled plasma-atomic emission spectroscopy (ICP-AES) for all 10 oxides simultaneously, or the oxides are determined individually by spectrophotometric and atomic absorption techniques (Shapiro, 1975). In the ICP-AES procedure, four Geological Survey rock standards are used to standardize the instrumentation, and indium is added to samples and standards as an internal control.

$$
\mathrm{FeO}, \mathrm{Cl}, \mathrm{F}, \mathrm{S}, \mathrm{CO}_{2} \text {, and } \mathrm{H}_{2} \mathrm{O} \pm
$$

These determinations are outlined more thoroughly in the previous sections. $\mathrm{FeO}$ is determined by titration with $\mathrm{K}_{2} \mathrm{Cr}_{2} \mathrm{O}_{7}$ after acid digestion of the sample. $\mathrm{Cl}$ and $\mathrm{F}$ are determined by using ISE or by IC. S is determined by using a Leco automated combustion analyzer. $\mathrm{CO}_{2}$, up to 0.1 percent, is determined volumetrically (Shapiro, 1975), and $\mathrm{CO}_{2}$, greater than 0.1 percent, is determined coulometrically. $\mathrm{H}_{2} \mathrm{O}-$ is determined gravimetrically. $\mathrm{H}_{2} \mathrm{O}+$ is determined by a modified Penfield method (Shapiro, 1975) or by an elemental analyzer

\section{Reporting of Results}

Constituents greater than or equal to 1 percent are reported to the nearest 0.1 percent. Constituents below 1 percent are reported to the nearest 0.01 percent.

\section{Discussion}

The rapid rock analysis scheme has evolved considerably since originally presented by Shapiro and Brannock (1952). The 10 major oxides are now determined routinely by ICP-AES. (See Chapter B on ICP-AES methods.) The ICP-AES procedure offers the advantage 
of simultaneous determination of the 10 oxides. Only occasionally are the major oxides determined by the spectrophotometric and atomic absorption spectroscopy methods described by Shapiro (1975) in his revised edition on the rapid analysis of silicate, carbonate, and phosphate rocks.

Determinations of $\mathrm{FeO}, \mathrm{H}_{2} \mathrm{O} \pm$, and $\mathrm{CO}_{2}$ also are included routinely in the rapid rock scheme. Summation of the 14 major and minor constituents usually is within 98.5-100 percent. When low summations occur (less than 98.5 percent), sulfur, fluoride, organic carbon, and volatiles other than $\mathrm{CO}_{2}$ and $\mathrm{H}_{2} \mathrm{O}$ are determined by loss on ignition at $1,000^{\circ} \mathrm{C}$, unless specific determination of those constituents is requested.

In the major oxide determinations, within-day precision is checked by the replicate analysis of samples, and day-to-day precision is checked by the periodic analysis of standards. Estimates of the relative precision are shown in table 10.

Table 10. Estimate of relative precision for rapid rock analysis [in percent]

\begin{tabular}{cc}
\hline $\begin{array}{c}\text { Concentration } \\
\text { range }\end{array}$ & $\begin{array}{c}\text { Relative } \\
\text { precision }\end{array}$ \\
\hline 10 & $1-2$ \\
$1-10$ & $2-10$ \\
$0.1-1$ & $10-20$ \\
$<0.1$ & $50-100$ \\
\hline
\end{tabular}

\section{REFERENCES CITED}

Abbey, S., 1983, Studies in "Standard Samples" of silicate rocks and minerals 1969-1982: Geological Survey of Canada Paper 83-15, 114 p.

Aruscavage, P. J., and Campbell, E. Y., 1983, An ion-selective electrode method for the determination of chlorine in geological materials: Talanta, v. 30, p. 745-749.

American Society for Testing and Materials, 1985, ASTM 0-2492-84, Standard test method for the forms of sulfur in coal, in 1985 annual book of American society for testing and materials standards, gaseous fuels, coal and coke, v. 5.05 , p. 256-260: Philadelphia, American Society for Testing and Materials, $572 \mathrm{p}$.
Bodkin, J. B., 1977, Determination of fluorine in silicates by use of an ion-selective electrode following fusion with lithium metaborate: Analyst, v. 102, p. 409-413.

Bowman, W. S., Faye, G. H., Sutarno, R., McKeague, J. A., and Kodama, H., 1979, Soil samples SO-1, SO-2, SO-3, and SO-4-Certified reference materials: Canadian Centre for Mineral and Energy Technology Report 79-3, 32 p.

Chalmers, R. A., and Page, E. S., 1957, The reporting of chemical analyses of silicate rocks: Geochimica et Cosmochimica Acta, v. 11, p. 247-251.

Cremer, M. J., and Elsheimer, H. N., 1972, Microcoulometric measurement of water in minerals: Analytica Chimica Acta, v. 60, p. 183-192.

Cremer, M. J., Klock, P. R., Neil, S. T., and Riviello, J. M., 1984, Chemical methods for the analysis of rocks and minerals: U.S. Geological Survey Open-File Report 84-565, 149 p.

Din, V. K., and Jones, G. C., 1978, The determination of total carbon and combined water in silicates using a $\mathrm{C}, \mathrm{H}, \mathrm{N}$ elemental analyser: Chemical Geology, v. 23, p. 347-352.

Engleman, E. E., Jackson, L. L., and Norton, D. R., 1985, Determination of carbonate carbon in geologic materials by coulometric titration: Chemical Geology, v. 53, p. 125-128.

Fishman, M. J., and Bradford, W. L., 1982, A supplement to methods for the determination inorganic substances in water and fluvial sediments: U.S. Geological Survey OpenFile Report 82-272, 136 p.

Flanagan, F. J., Chandler, J. C., Breger, I. A., Moore, C. B., and Lewis, C. F., 1976, The carbon content of U.S. Geological Survey volcanic rock standards, in Flanagan, F. J., ed., Descriptions and analyses of eight new U.S. Geological Survey rock standards: U.S. Geological Survey Professional Paper 840, p. 123-126.

Flanagan, F. J., and Kirschenbaum, Herbert, 1984, The precision of classical rock analysis: Geostandards Newsletter, v. 8, p. 7-11.

Gladney, E. S., Burns, C. E., Perrin, D. R., Roelandts, I., and Gills, T. E., 1984, 1982 compilation of elemental concentration data for NBS biological, geological, and environmental standard reference materials: National Bureau of Standards Special Publication 260-88, 221 p.

Greenland, L. P., 1962, The separation of fluorine by microdiffusion of hydrofluoric acid: Analytica Chimica Acta, v. 27, p. 386-389.

-1963, Fractionation of chlorine, germanium, and zinc in chondritic meteorites: Journal of Geophysical Research, v. 68 , p. 6507-6513.

Groves, A. W., 1951, Silicate analysis [2d ed.]: London, Allen and Unwin Ltd., $336 \mathrm{p}$.

Hillebrand, W. F., 1919, The analysis of silicate and carbonate rocks: U.S. Geological Survey Bulletin 700, 285 p.

Hillebrand, W. F., Lundell, G. E. F., Bright, H. A., and Hoffman, J. I., 1953, Applied inorganic analysis [2d ed.]: New York, Wiley and Sons, 1034 p.

Hopkins, D. M., 1977, An improved ion-selective electrode method for the rapid determination of fluorine in rocks and soils: U.S. Geological Survey Journal of Research, v. 5, no. 5 , p. 589-593.

Huang, W. H., and Johns, W. D., 1967, The chlorine and 
fluorine contents of geochemical standards: Geochimica et Cosmochimica Acta, v. 31, p. 597-602.

Ingamells, C. O., 1960, A new method for "ferrous iron" and "excess oxygen" in rocks, minerals, and oxides: Talanta, v. 4, p. 268-273.

Ingram, B. L., 1970, Determination of fluoride in silicate rocks without separation of aluminum using a specific ion electrode: Analytical Chemistry, v. 42, p. 1825-1827.

Jackson, L. L., Engleman, E. E., and Peard, J. L., 1985a, Determination of total sulfur in lichens and plants by combustion-infrared analysis: Environmental Science Technology, v. 19, p. 437-441.

Jackson, L. L., Engleman, E. E., and Severson, R. C., 1985b, Characterization of forms of sulfur in overburden materials of the Western United States: The 2nd Annual Meeting, American Society for Surface Mining and Reclamation, Denver, CO, 1985, Proceedings, p. A1-A4.

Jackson, L. L., Taggart, J. E., Bartel, A. J., Stewart, K. C., Engleman, E. E., and Brandt, E. L., 1984, Comparison of $\mathrm{X}$-ray fluorescence spectroscopy and classical chemical techniques for the determination of major elements in geological materials: presented at Denver Conference on Applications of X-ray Analysis, Denver, CO.

Jackson, L. L., Taggart, J. E., and Foord, E. E., 1985c, Quantitative determination of water in small mineral samples: Pittsburgh Conference, New Orleans, LA, 1985, Abstract 1191.

Jeffery, P. G., and Hutchison, D., 1981, Chemical methods of rock analysis [3rd ed.]: New York, Pergamon Press, 379 p.

Johnson, W. M., and Maxwell, J. A., 1981, Rock and mineral analysis [2nd ed.]: New York, Wiley, 489 p.

Kaplan, I. R., Emery, K. O., and Rittenberg, S. C., 1963, The distribution and isotopic abundance of sulphur in recent marine sediments of southern California: Geochimica et Cosmochimica Acta, v. 27, p. 297-331.

Karr, C., 1979, Analytical methods for coal and coal products, v. III: New York, Academic Press, 641 p.

Kirschenbaum, Herbert, 1983, The classical chemical analysis of silicate rocks-The old and the new: U.S. Geological Survey Bulletin 1547, 55 p.

Krauskopf, K. B., 1979, Introduction to geochemistry [2nd ed.]: New York, McGraw-Hill Book Company, 617 p.

Leventhal, J. S., and Shaw, V. E., 1980, Organic matter in Appalachian Devonian black shale-pt. I, Comparison of techniques to measure organic carbon, pt. II, Short range organic carbon content variations: Journal of Sedimentary Petrology, v. 50, p. 77-81.

Long, G. L., and Winefordner, J. D., 1983, Limit of detection: A closer look at the IUPAC definition: Analytical Chemistry, v. 55 , p. $712 \mathrm{~A}-724 \mathrm{~A}$.

Manheim, F. T., Hathaway, J. C., Flanagan, F. J., and Fletcher, J. D., 1976, Marine mud, MAG-1, from the Gulf of Maine, in Flanagan F. J., ed., Descriptions and analyses of eight new USGS rock standards: U.S. Geological Survey Professional Paper 840, p. 25-28.
Maxwell, J. A., 1968, Rock and mineral analysis: New York, Wiley and Sons, $584 \mathrm{p}$

Nelson, D. W., and Sommers, L. E., 1982, Total carbon, organic carbon, and organic matter, pt. 2, in Page, A. L., Miller, R. H., and Keeney, D. R., eds, Methods of soil analysis [2nd ed.],: Madison, Soil Science Society of America, 1159 p.

Nicholson, K., 1983, Fluorine determination in geochemistry: Errors in the electrode method of analysis: Chemical Geology, v. 38, p. 1-22.

Norton, D. R., 1982, The coulometric determination of moisture and combined water in silicate rocks using the Karl Fischer titration method: Rocky Mountain Conference, Denver, CO., Abstract 243.

Norton, D. R., and Engleman, E. E., 1980, The coulometric determination of carbon dioxide in geologic materials: Rocky Mountain Conference, Denver, CO., Abstract 105.

Peck, L. C., 1964, Systematic analysis of silicates: U.S. Geological Survey Bulletin 1170, 89 p.

Penfield, S. L., 1894, On some methods for the determination of water: American Journal of Science, v. 48, no. 283, p. 30-37.

Rice, T. D., 1976, An interlaboratory study of potassium determination in rocks and minerals: Talanta, v. 23, p. 359-367.

Scholz, A., and Rathleff, D., 1982, A rapid method to determine total sulfur: Journal of Coal Quality, v. 2, p. 16-19.

Searle, P. L., 1968, Determination of total sulfur in soil by using high-frequency induction furnace equipment: Analyst, v. 93 , p. $540-545$.

Shapiro, Leonard, 1975, Rapid analysis of silicate, carbonate, and phosphate rocks [revised ed.]: U.S. Geological Survey Bulletin 1401, 76 p.

Shapiro, Leonard, and Brannock, W. W., 1952, Rapid analysis of silicate rocks: U.S. Geological Survey Circular 165, 17 p.

Skinner, N. G., Brown, F. W., and Flanagan, F. J., 1981, The $\mathrm{H}_{2} \mathrm{O}+$ contents of some geochemical standards predicted by a calibration line: Geostandards Newsletter, v. 5, p. 3-11.

Small, H., Stevens, T. S., and Bauman, W. C., 1975, Novel ion exchange chromatographic method using conductimetric detection: Analytical Chemistry, v. 47, p. 1801-1809.

Smith, J. L., 1871, On the determination of the alkalis in silicates by ignition with carbonate of lime and salammoniac: American Journal of Science, ser. 3, v. 1, no. 4, p. 269-275.

Sobek, A. A., and Bogner, J. E., 1984, Using the acid-base account to predict acid potential of coal overburden: The Third Biennial Symposium on Surface Coal Mine Reclamation on the Great Plains, Billings, MT, 1984, Proceedings, p. A1-A21.

Stanton, R. W., and Renton, J. J., 1981, Organic and pyritic sulfur in coal-Potential errors in determination: West Virginia Geological and Economic Survey Circular No. C-22, 13 p. 
Thomas, J. T., and Gluskoter, H. J., 1974, Determination of fluoride in coal with the fluoride ion-selective electrode: Analytical Chemistry, v. 46, p. 1321-1323.

Tillman, J. H., 1977, A combustimetric method for determining the total carbon content of geologic materials: U.S. Geological Journal of Research, v. 5, no. 5, p. 583-587.

Todor, D. N., 1976, Thermal analysis of minerals: England, Abacus Press, 256 p.
Washington, H. S., 1904, Manual of the chemical analysis of rocks: New York, Wiley and Sons, $183 \mathrm{p}$.

Wilson, S. A., and Gent, C. A., 1982, The determination of fluoride in geologic samples by ion chromatography: Analytical Letters, v. 15, p. 851-864.

1983, Determination of chloride in geological samples by ion chromatography: Analytica Chimica Acta, v. 148, p. 299-303. 
CHAPTER $\mathrm{H}$

Instrumental Neutron Activation Analysis of Geochemical Samples

By P. A. BAEDECKER and D. M. MCKOWN

U.S. GEOLOGICAL SURVEY BULLETIN 1770

Methods for Geochemical Analysis 



\section{CONTENTS}

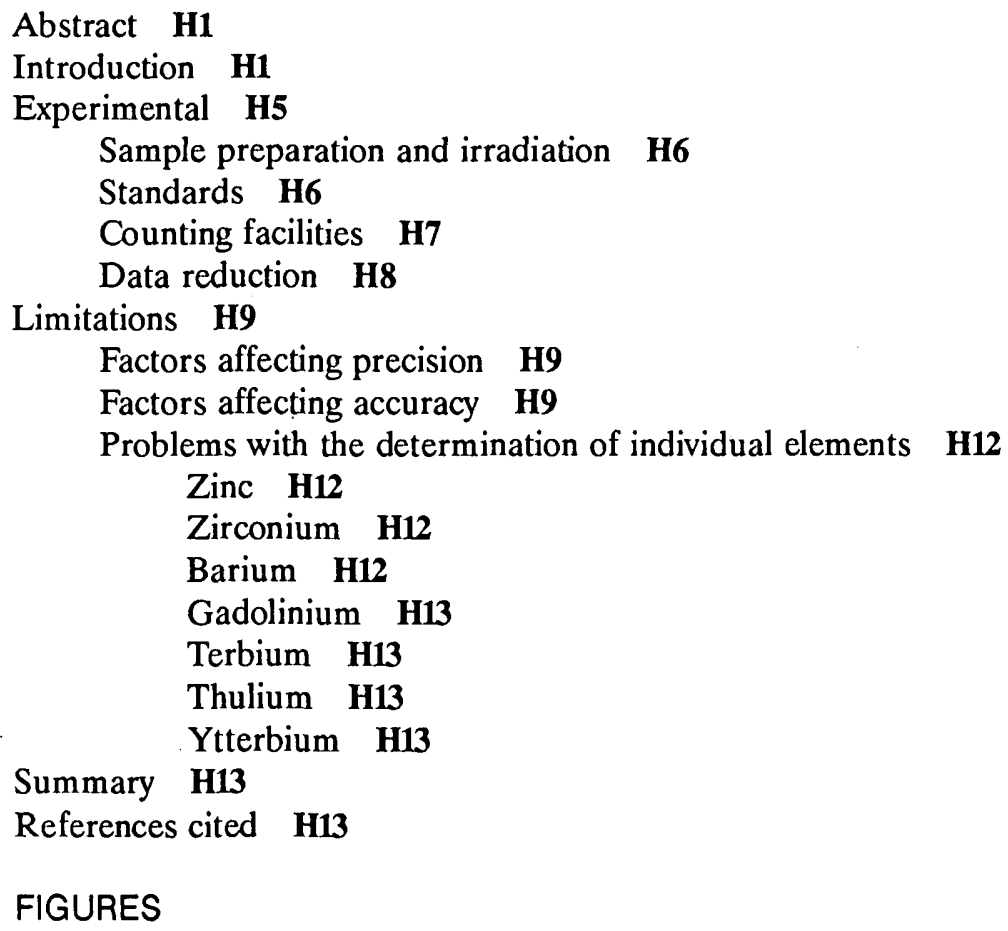

FIGURES

1. Germanium detector gamma-ray spectrum of a neutron activated rhyolite 10 days after irradiation $\mathbf{H} 2$

2. Low-Energy Photon Detector gamma-ray spectrum of a neutron activated rhyolite 60 days after irradiation $\mathbf{H 3}$

3. Germanium detector spectra of a neutron activated basalt sample irradiated with and without a cadmium cover (10 days decay) $\mathbf{H 8}$

\section{TABLES}

1. Long-lived indicator radionuclides $\mathbf{H 4}$

2. Short-lived indicator radionuclides $\mathbf{H 7}$

3. Precision of the determination of 23 elements in basalt BCR-1 and granite G-2 by instrumental neutron activation analysis. H10

4. Interferences in instrumental neutron activation analysis due to neutron-induced fission of uranium-235 H11

5. Instrumental neutron activation analysis of silicate rocks H14 



\title{
Instrumental Neutron Activation Analysis of Geochemical Samples
}

\author{
By P. A. Baedecker and D. M. McKown
}

\begin{abstract}
Instrumental neutron activation analysis with thermal neutrons is a well-tested method for the determination of up to 30 elements in geological samples. The method is based on the irradiation of samples and standards in a reactor neutron flux and the measurement of the induced radioactivity using high-resolution gamma-ray spectrometry. The technique has good sensitivity (0.1-10 parts per million) for a wide range of elements, including many of the first-row transition elements, rare earths, alkali, and alkaline earths. When coupled with $X$-ray fluorescence analysis, the two techniques provide excellent data for studying minor variations among geochemically coherent pairs of elements; for example, yttrium and the rare-earth elements, zirconiumhafnium, niobium-tantalum, uranium-thorium. The geochemical applications of the technique have been in such diverse areas as the study of trace-element variations in igneous and metamorphic petrology, the characterization of coals for their contents of potentially deleterious trace elements (for example, zinc, arsenic, selenium, antimony, mercury), and the analysis of plant material as biogeochemical indicators of ore deposits (tungsten and gold).
\end{abstract}

\section{INTRODUCTION}

Instrumental activation analysis with thermal neutrons (INAA) is a versatile technique for elemental analysis because it has high sensitivity for many elements, lends itself to automation, and provides precise data for many major, minor, and trace elements in a single sample aliquant without chemical treatment. The application of INAA to the analysis of geological samples has been described in a number of publications (for example, Gordon and others, 1968; Hertogen and Gijbels, 1971; Baedecker and others, 1977; Jacobs and others, 1977; Laul, 1979). By restricting the analysis to the measurement of long-lived nuclides ( $\left.t_{1 / 2}>1 \mathrm{~d}\right), 25$ elements can be determined routinely in most rock samples (29 elements are determined routinely in coal). By extending the technique to the measurement of shorter lived activities, with rapid sample transfer and short irradiation and counting times, an additional seven elements can be determined.
Activation analysis is based on the selective measurement of the activity from radioactive nuclides that are produced by nuclear reactions on naturally occurring isotopes of the element to be determined in the sample. Reactor neutrons are used most commonly for inducing the nuclear transformations because of their availability, the relatively high probability for thermal neutron-induced reactions, and the relative freedom from problems due to matrix effects (self shielding); for example, the determination of $\mathrm{La}$ is carried out by irradiating the sample in a reactor neutron flux to induce the following reaction:

$$
{ }^{139} \mathrm{La} \text { (stable) }+\mathrm{n} \rightarrow{ }^{140} \mathrm{La} \text { (radioactive) }+\gamma,
$$

where the amount of $\mathrm{La}$ in the sample is determined by measuring the induced ${ }^{140} \mathrm{La}$ activity. The activity of the indicator radionuclide produced during the irradiation is directly proportional to the amount of element of interest in the sample, and the analytical determination generally is made by comparing the activity induced in the sample against the activity measured for well-characterized standard samples. The activated ${ }^{140} \mathrm{La}$ subsequently decays by the following reaction:

$$
{ }^{140} \mathrm{La}\left(\mathrm{t}_{1 / 2}=40.3 \mathrm{~h}\right) \rightarrow{ }^{140} \mathrm{Ce}+\beta^{-}+\gamma,
$$

with a characteristic half-life $\left(t_{1 / 2}\right)$ and spectrum of gamma-ray energies that facilitate the selective identification and measurement of the induced radioactivity after the sample has been removed from the irradiation facility. The activities of the samples and standards are assayed most commonly by gamma-ray spectroscopy because potentially interfering activities can often be discriminated against by looking at gamma-rays having unique energies for the indicator radionuclide and because gamma counting is relatively free from matrix effects (self absorption). Semiconductor detectors, such as highpurity germanium and lithium drifted germanium $(\mathrm{Ge}(\mathrm{Li}))$ diodes, generally are used for gamma-ray spectroscopy because of their excellent resolution that permits the separation of closely spaced lines. These devices act as transducers to convert the gamma-ray signal from the 
irradiated samples to electronic pulses that then can be sorted according to amplitude by an analog to digital converter. The pulses within each amplitude interval are counted by a multichannel scaler (each channel corresponding to a separate interval of gamma-ray energy). A typical gamma-ray spectrum from an irradiated rhyolite (U.S. Geological Survey reference sample RGM-1), measured 1 week following irradiation, is shown in figure 1 . The radioactivity of any radionuclide of interest in the sample is measured selectively by determining the area of a specific gamma-ray photopeak of interest above an underlying continuum. The region of the gamma-ray spectrum below $150 \mathrm{keV}$ is generally quite complex, and it is frequently advantageous to count the samples with a thin germanium diode detector that has higher resolution in this low-energy region of the spectrum and that is relatively transparent to higher energy gamma rays. This detector, referred to as a Low-Energy Photon Detector, or LEPD, supplements the data acquired from standard Ge detector counting by providing data from alternate lines for the elements $\mathrm{Ba}, \mathrm{Nd}, \mathrm{Sm}, \mathrm{Tb}, \mathrm{Hf}, \mathrm{Th}$, and $\mathrm{U}$ and is the only means of acquiring data for $\mathrm{Gd}, \mathrm{Ho}$, and $\mathrm{Tm}$. A typical LEPD spectrum of RGM-1 2 mo after irradiation is shown in figure 2.

A further degree of selectivity can be derived from the relative differences in the half-lives (decay rates) of individual activation products. Thus, optimum results generally are obtained by counting at various time intervals following the irradiation of the sample-early for relatively short-lived species and at longer decay times for long-lived isotopes, after spectral components from shorter lived species have had time to decay away.

If only relatively long-lived $\left(t_{1 / 2}>1 \mathrm{~d}\right)$ indicator radionuclides are employed in the analysis, then up to 25 elements can be determined by using long irradiation times ( $>/=8 \mathrm{~h}$ at a neutron flux of $3 \times 10^{12}$ neutrons $\mathrm{cm}^{-2} \mathrm{~s}^{-1}$ ) and multiple sample counts during the decay period between $7 \mathrm{~d}$ and 2 mo following the irradiation. A list of the elements, their indicator radionuclides, halflives, gamma-ray lines, detection limits, and potential spectral interferences are given in table 1; advantage factors for epithermal neutron activation, relative to thermal activation, also are listed. The pertinent information for the short-lived species that can be measured for the determination of an additional seven elements are listed in table 2. Those with half-lives of less than $1 \mathrm{~h}$ generally are measured after an irradiation of $1 \mathrm{~min}$ at a lower flux position in the U.S. Geological Survey Triga reactor and are counted after approximately $10 \mathrm{~min}$ decay. $\mathrm{Mn}$ and Dy are measured after a $15-\mathrm{min}$ irradiation (at $3 \times 10^{12}$ neutrons $\mathrm{cm}^{-2} \mathrm{~s}^{-1}$ ) and approximately $3 \mathrm{hr}$ decay.

The estimation of detection limits for INAA is subject to considerable uncertainty because it is dependent on the signal to background ratio for each photopeak of each sample being counted and on the characteristics of the $\mathrm{Ge}$ detector employed, where the Compton continuum from higher energy gamma-rays contributes to the background. The detection limits, therefore, are dependent on sample composition, principally the contents of

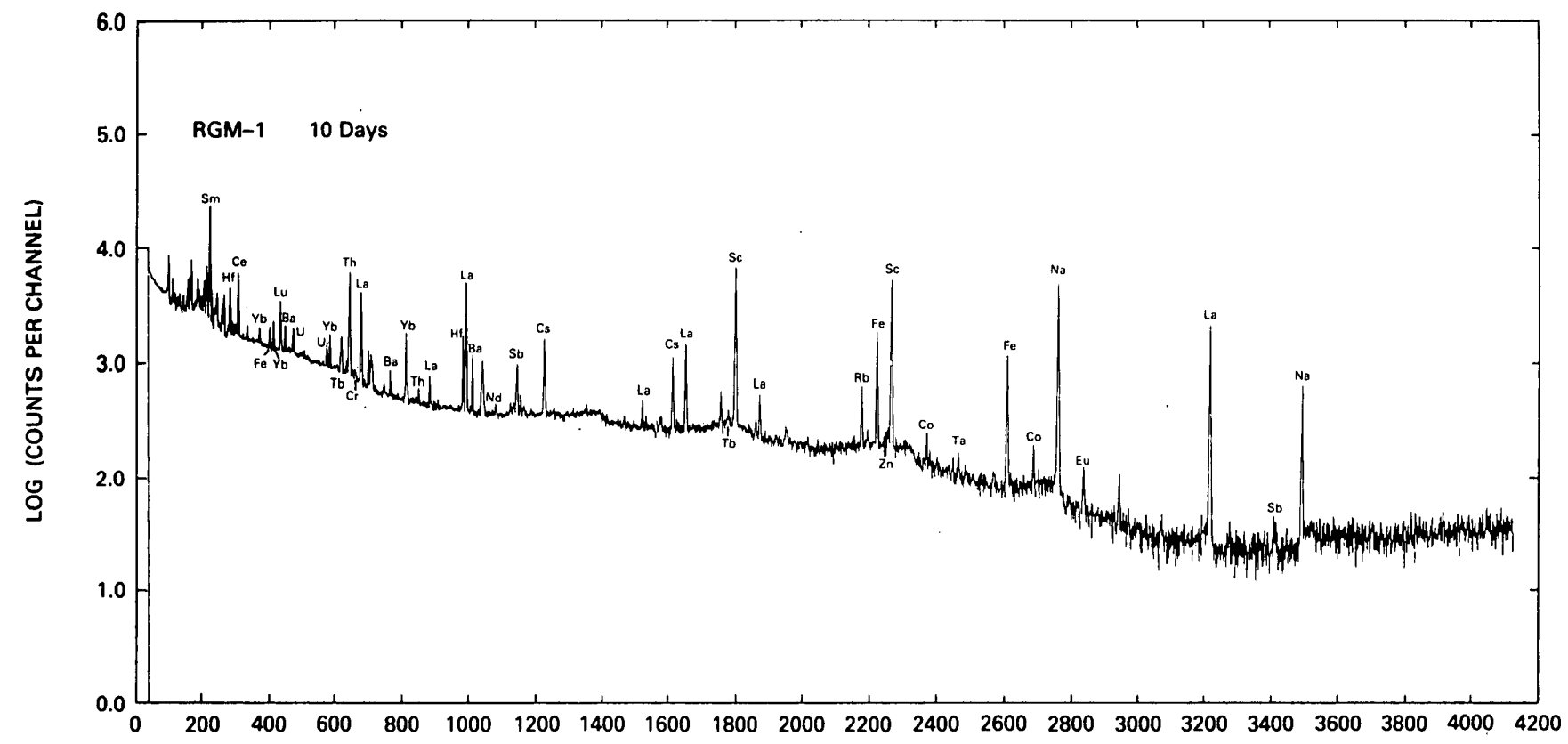

CHANNEL NUMBER

Figure 1. Germanium detector gamma-ray spectrum of a neutron activated rhyolite (U.S. Geological Survey reference sample rhyolite RGM-1) 10 days after irradiation. 


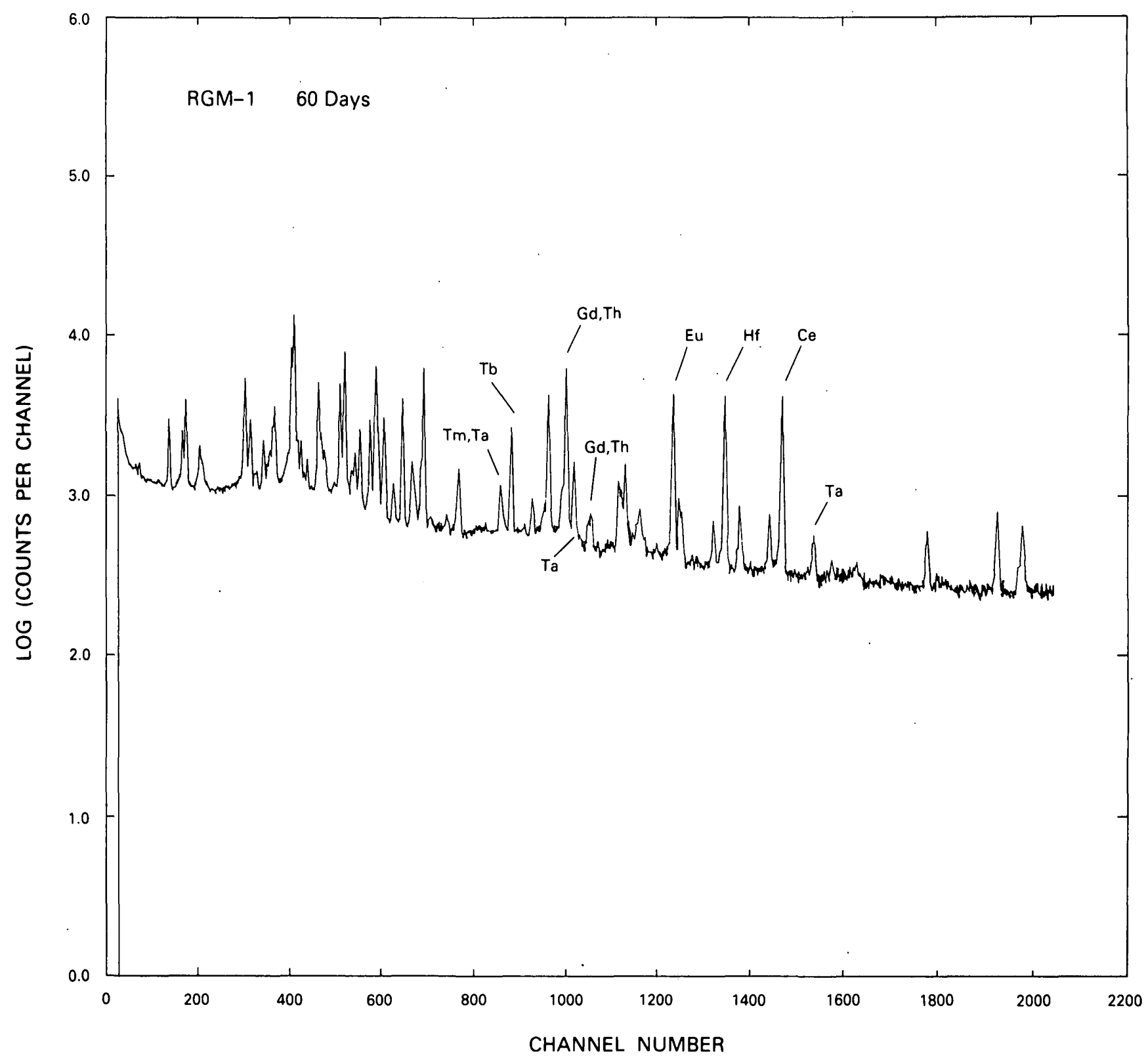

Figure 2. Low-Energy Photon Detector gamma-ray spectrum of a neutron activated rhyolite (U.S. Geological Survey reference sample $R G M-1) 60$ days after irradiation.

$\mathrm{Na}, \mathrm{Sc}, \mathrm{Fe}, \mathrm{Co}$, and La because activation products from these elements tend to dominate the spectrum of most activated rock samples following a few days decay and to limit the sensitivity for the determination of other elements. Therefore, the tabulated detection limits are for a rock sample of granitic composition; for example, reference sample granite G-2. Detection limits for a basalt would tend to be higher by roughly a factor of four due to the higher contents of Sc and Fe but could be an order of magnitude higher for any given sample based on various factors; for example, spectral interferences due to composition and counting statistics as controlled by irradiation, decay time, and counting parameters.
Irradiation with epithermal neutrons (ENAA), rather than the entire reactor spectrum, is advantageous in the cases where an element is determined using a nuclide with a high $I$ to $\sigma_{\mathrm{o}}$ (resonance activation integral-thermal neutron cross section) ratio as compared with the nuclides giving rise to the major activities in the sample. Epithermal irradiations are performed by filtering out thermal neutrons by enclosing the samples in a $\mathrm{Cd}$ container during irradiation because ${ }^{113} \mathrm{Cd}$ has an exceptionally high cross section for thermal neutrons (approximately $2 \times 10^{4}$ barns). The method has been shown to be particularly suitable for the analysis of silicate rocks because many of the major activities from thermal neu- 
Table 1. Long-lived $\left(t_{1 / 2}>10\right.$ hours) indicator radionuclides [h, hour; d, day; $y$, year]

\begin{tabular}{|c|c|c|c|c|c|c|c|c|c|}
\hline \multirow[t]{2}{*}{ Element } & \multirow{2}{*}{$\begin{array}{l}\text { Indicator } \\
\text { radionuclide }\end{array}$} & \multirow{2}{*}{$\begin{array}{l}\text { Half- } \\
\text { life }\end{array}$} & \multirow{2}{*}{$\begin{array}{c}\text { Preferred } \\
\text { gamma-ray } \\
\text { energy (keV) } 1\end{array}$} & \multirow{2}{*}{$\begin{array}{l}\text { Linit of } \\
\text { deternination }\end{array}$} & \multirow{2}{*}{$\begin{array}{l}\text { ENAA }^{3} \\
\text { advantage } \\
\text { factor }\end{array}$} & \multicolumn{2}{|c|}{ Potential Spectral } & \multicolumn{2}{|c|}{ Interferences } \\
\hline & & & & & & $\begin{array}{l}\text { Radio- } \\
\text { nuclide }\end{array}$ & $\begin{array}{r}\text { Energy } \\
(\mathrm{keV})\end{array}$ & $\begin{array}{l}\text { Radio- } \\
\text { nuclide }\end{array}$ & $\begin{array}{r}\text { Energy } \\
\text { (keV) }\end{array}$ \\
\hline $\mathrm{Na}$ & ${ }^{24} \mathrm{Na}$ & $15.0 \mathrm{~h}$ & 1368.6 & 10 & 1.2 & & & . & \\
\hline & & & 2753.9 & & & & & & \\
\hline K & $42_{K}$ & $12.4 \mathrm{~h}$ & 1524.7 & 0.01 percent & 2.0 & & & & \\
\hline Sc & ${ }^{46} \mathrm{SC}$ & $84 d$ & 1120.5 & 0.01 & 1.0 & ${ }^{182} \mathrm{Ta}$ & 1121.3 & & \\
\hline $\mathrm{Cr}$ & ${ }^{51} \mathrm{Cr}$ & $27.8 \mathrm{~d}$ & 320.1 & 0.5 & 1.6 & ${ }^{177} 7_{L u}$ & 321.3 & 147 Nd & 319.4 \\
\hline $\mathrm{Fe}$ & ${ }^{59} \mathrm{Fe}$ & $45.6 \mathrm{~d}$ & 1099.3 & 50 & 2.3 & ${ }^{182} \mathrm{Ta}$ & 1289.1 & & \\
\hline & & & 1291.5 & 75 & & & & & \\
\hline Co & ${ }^{60} \mathrm{CO}_{\mathrm{O}}$ & $5.3 y$ & 1173.2 & 0.2 & 4.4 & & & & \\
\hline & & & 1332.5 & & & & & & \\
\hline $\mathrm{Mi}$ & ${ }^{58} \mathrm{Co}$ & $71.0 \mathrm{~d}$ & 810.8 & 50 & & & & & \\
\hline $2 n$ & ${ }^{65} \mathrm{zn}$ & $245 d$ & 1115.4 & 1 & 3.8 & ${ }^{160}{ }_{\mathrm{Tb}}$ & 1115.1 & & \\
\hline $\mathrm{Rb}$ & ${ }^{86_{R b}}$ & $18.7 d$ & 1076.8 & 5 & 24 & & & & \\
\hline $\mathrm{Sr}$ & ${ }^{85} s r$ & $64.0 d$ & 514.0 & 50 & 21 & & & & \\
\hline $2 \mathbf{r}$ & ${ }^{95} \mathrm{Nb}$ & $35.1 \mathrm{~d}$ & 765.8 & 200 & & ${ }^{152}$ EU & 764.8 & ${ }^{160}{ }_{\mathrm{Tb}}$ & 765.3 \\
\hline Mo & ${ }^{99}{ }_{\text {Mo }}$ & $66.7 \mathrm{~h}$ & 140.5 & 10 & 44 & ${ }^{59} \mathrm{Fe}$ & 142.7 & & \\
\hline Sb & ${ }^{124} \mathrm{Sb}$ & $60.0 \mathrm{~d}$ & 1691.0 & 0.1 & 46 & & & & \\
\hline & ${ }^{122}$ Sb & $67.2 \mathrm{~h}$ & 564.0 & 0.1 & 34 & & & & \\
\hline
\end{tabular}

tron activation show low ratios (Brunfelt and Steinnes, 1969). The advantage of epithermal activation is illustrated in figure 3, which shows Ge spectra for a basalt sample containing $52 \mathrm{ppm} \mathrm{Sc}$ irradiated with and without a Cd filter. The two photopeaks of ${ }^{46} \mathrm{Sc}$ dominate both spectra and the Compton background (the broad continuum below the photopeaks) due to ${ }^{46} \mathrm{Sc}$ limits the detection limits for most of the other elements of interest. However, epithermal irradiation enhances a number of photopeaks relative to the Sc activity. As a supplement to the standard INAA technique, ENAA is advantageous for only about one-half of the elements that normally are determined by INAA. For epithermal irradiation, "advantage factors," which are the I to $\sigma_{\mathrm{o}}$ ratio for the production of a given radionuclide relative to a neutron absorber (such as ${ }^{46} \mathrm{Sc}$ ) whose cross section falls off with increasing neutron energy and has no high-resonance cross sections at higher neutron energies, can be calculated. Advantage factors for most of the nuclei of interest in the ENAA analysis of silicate rocks are listed in table 1 . The elements $\mathrm{Sr}, \mathrm{Mo}, \mathrm{W}$, and $\mathrm{Au}$ are usually below INAA sensitivity limits in most rock samples, but information for those elements is included in table 1 because they often can be determined by ENAA. The relative merits of epithermal and thermal instrumental neutron activation of silicate rocks have been reviewed by Baedecker and others (1977).

A similar enhancement of low-energy lines relative to an underlying Compton continuum also can be achieved by a special Compton suppression counting 
Table 1. Long-lived $\left(t_{1 / 2}>10\right.$ hours) indicator radionuclides-Continued

\begin{tabular}{|c|c|c|c|c|c|c|c|c|c|}
\hline \multirow[t]{2}{*}{ Element } & \multirow{2}{*}{$\begin{array}{l}\text { Indicator } \\
\text { radionuclide }\end{array}$} & \multirow{2}{*}{$\begin{array}{l}\text { Half- } \\
\text { life }\end{array}$} & \multirow{2}{*}{$\begin{array}{c}\text { Preferred } \\
\text { ganma-ray } \\
\text { energy (keV) } 1\end{array}$} & \multirow{2}{*}{$\begin{array}{l}\text { Limit of } \\
\text { deternination }\end{array}$} & \multirow{2}{*}{$\begin{array}{l}\text { ENAA }^{3} \\
\text { advantage } \\
\text { factor }\end{array}$} & \multicolumn{2}{|c|}{ Potential Spectral } & \multicolumn{2}{|c|}{ Interferences } \\
\hline & & & & & & $\begin{array}{l}\text { Radio- } \\
\text { nuclide }\end{array}$ & $\begin{array}{r}\text { Energy } \\
(\mathrm{keV})\end{array}$ & $\begin{array}{l}\text { Radio- } \\
\text { nuclide }\end{array}$ & $\begin{array}{l}\text { Energy } \\
\text { (keV) }\end{array}$ \\
\hline Cs & ${ }^{134} \mathrm{Cs}$ & $2.1 \mathrm{y}$ & 795.8 & 0.1 & 22 & & & & \\
\hline \multirow[t]{2}{*}{$\mathrm{Ba}$} & $131_{\mathrm{Ba}}$ & $12.0 \mathrm{~d}$ & 496.3 & 100 & 32 & ${ }^{103} \mathrm{Ru}$ & 497.0 & & \\
\hline & & & 123.7 & 200 & & & & & \\
\hline \multirow[t]{2}{*}{ La } & $140 \mathrm{La}$ & $40.2 \mathrm{~h}$ & 1596.6 & 0.02 & 2.9 & & & & \\
\hline & & & 487.0 & 0.05 & & & & & \\
\hline Ce & ${ }^{141} \mathrm{Ce}$ & $32.5 \mathrm{~d}$ & 145.4 & 0.5 & 1.8 & ${ }^{175} \mathrm{Yb}$ & 144.8 & & \\
\hline \multirow[t]{2}{*}{ Nd } & ${ }^{147}$ Nd & $11.1 \mathrm{~d}$ & 531.0 & 2 & 4.9 & & & & \\
\hline & & & 91.1 & 5 & & & & & \\
\hline \multirow[t]{2}{*}{ Sn } & ${ }^{153} \mathrm{Sw}$ & $46.8 \mathrm{~h}$ & 103.2 & 0.5 & 20 & ${ }^{239}{ }_{\mathrm{Np}}$ & 103.7 & & \\
\hline & & & 69.7 & 0.2 & & & & & \\
\hline \multirow[t]{3}{*}{ Eu } & ${ }^{152} \mathrm{Eu}$ & $12.7 \mathrm{y}$ & 779.1 & 0.04 & 8.1 & & & & \\
\hline & & & 1408.1 & 0.01 & & & & & \\
\hline & & & 121.8 & 0.02 & & ${ }^{75} \mathrm{Se}$ & 121.1 & & \\
\hline Gd & ${ }^{153}$ Gd & $242.0 d$ & 103.2 & 2.0 & 5.9 & & & & \\
\hline \multirow[t]{2}{*}{ Tb } & ${ }^{160}$ Tb & $72.1 \mathrm{~d}$ & 298.6 & 0.05 & 25 & ${ }^{233} \mathrm{~Pa}$ & 299.9 & & \\
\hline & & & 1178.1 & 0.1 & & . & & & \\
\hline Ta & ${ }^{170}{ }_{\mathrm{Ta}}$ & $127.0 \mathrm{~d}$ & 84.3 & 0.5 & 22 & & & & \\
\hline \multirow[t]{2}{*}{$\mathrm{Yb}$} & ${ }^{175}{ }_{\mathrm{Yb}}$ & $101.0 \mathrm{~h}$ & 396.1 & 0.1 & 10 & ${ }^{147}$ Nd & 398.2 & ${ }^{233} \mathrm{~Pa}$ & 398.2 \\
\hline & & & 282.6 & 0.2 & & & & & \\
\hline Lu & $177_{\text {Lu }}$ & $6.7 d$ & 208.4 & 0.01 & $<1$ & ${ }^{239} \mathrm{~Np}$ & 209.7 & & \\
\hline
\end{tabular}

system, which involves surrounding a normal $\mathrm{Ge}$ diode with an annulus $\mathrm{NaI}$ scintillation detector. Because a Compton event is caused by a photon imparting only a portion of its energy to the Ge diode, the photon from a Compton interaction also may be detected by the $\mathrm{NaI}$ annulus. If the data from the $\mathrm{Ge}$ and $\mathrm{NaI}$ detectors are acquired in an anticoincidence mode (simultaneous events recorded by both detectors are discarded), then the Compton background in the $\mathrm{Ge}$ detector spectrum can be reduced, resulting in an improved signal to background ratio for many photopeaks. Such systems are expensive and cannot be applied routinely for INAA experiments, but such a system is available in the Geological Survey's Denver laboratories for special applications.

\section{EXPERIMENTAL}

INAA involves a direct assay of an irradiated whole rock sample without any preirradiation or postirradiation chemical processing. Thus, the technique is inherently free of error caused by laboratory contamination. However, the experimental procedure, including sample preparation and the selection of irradiation and counting parameters, is dependent on a number of factors relevant to the experiment-the specific element(s) of interest, the composition of the matrix, the amount of sample available, and the data confidence required. For the analysis of most silicate rocks, the following generalized procedure is used. 
Table 1. Long-lived ( $t_{1 / 2}>10$ hours) indicator radionuclides-Continued

\begin{tabular}{|c|c|c|c|c|c|c|c|c|}
\hline \multirow[t]{2}{*}{ Elenent } & \multirow{2}{*}{$\begin{array}{l}\text { Indicator } \\
\text { radionuclide }\end{array}$} & \multirow{2}{*}{$\begin{array}{l}\text { Half- } \\
\text { life }\end{array}$} & \multirow{2}{*}{$\begin{array}{c}\text { Preferred } \\
\text { gamma-ray } \\
\text { energy (keV) } 1\end{array}$} & \multirow{2}{*}{$\begin{array}{l}\text { Linit of } \\
\text { deternination }\end{array}$} & \multirow{2}{*}{$\begin{array}{l}\text { ENAA }^{3} \\
\text { advantage } \\
\text { factor }\end{array}$} & \multicolumn{3}{|c|}{ Potential Spectral Interferences } \\
\hline & & & & & & $\begin{array}{l}\text { Radio- } \\
\text { nuclide }\end{array}$ & $\begin{array}{r}\text { Energy } \\
(\mathrm{keV})\end{array}$ & $\begin{array}{l}\text { Radio- Energy } \\
\text { nuclide (keV) }\end{array}$ \\
\hline \multirow[t]{2}{*}{ Hf } & ${ }^{181_{H f}}$ & $42.5 d$ & 482.2 & 0.1 & 5.4 & & & \\
\hline & & & 133.1 & 0.05 & & & & \\
\hline \multirow[t]{3}{*}{ Ta } & ${ }^{182} \mathrm{Ta}$ & $115.1 d$ & 1221.3 & 0.02 & 37 & & & \\
\hline & & & 1189.2 & 0.03 & & & & \\
\hline & & & 152.4 & 0.4 & & & & \\
\hline \multirow[t]{2}{*}{$W$} & ${ }^{187_{W}}$ & $24.0 \mathrm{~h}$ & 479.5 & 0.1 & 22 & & & \\
\hline & & & 685.7 & 0.1 & & ${ }^{147}$ Nd & 685.9 & \\
\hline Au & ${ }^{198} \mathrm{Au}$ & $2.7 d$ & 411.8 & 0.005 & 24 & ${ }^{152} \mathrm{Eu}$ & 411.1 & \\
\hline Th & ${ }^{233} \mathrm{~Pa}$ & $27.0 \mathrm{~d}$ & 311.9 & 0.1 & 19 & & & \\
\hline$u$ & ${ }^{239}{ }_{\mathrm{Hp}}$ & $2.3 \mathrm{~d}$ & 277.6 & 0.5 & 55 & & & \\
\hline
\end{tabular}

1keV: thous and electron-volts.

${ }^{2}$ Parts Der aillion except percent as indicated.

ENAA: Epithermal neutron activation analys is.

\section{Sample Preparation and Irradiation}

Powdered sample and standard aliquants $(0.5-1 \mathrm{~g})$ are weighed into polyethylene vials and heat sealed. Groups of sample and standard vials [33-37 samples plus 2-5 standards plus 1-2 Geological Survey standard rocks (as controls)] are simultaneously irradiated in a homogeneous neutron flux (up to $2 \times 10^{12}$ neutrons $\mathrm{cm}^{-2} \mathrm{~s}^{-1}$ ) using the Geological Survey's TRIGA reactor. Three separate irradiations would be required for the full suite of elements that can be determined by INAA. For the analysis of those elements with relatively long-lived indicator radionuclides $\left(t_{1 / 2}>10 \mathrm{hr}\right.$; table 1$)$, the samples and the standards are irradiated for $8 \mathrm{~h}$

Samples for epithermal activation are prepared by wrapping approximately $50 \mathrm{mg}$ of powdered sample in 3by $3-\mathrm{cm}$ squares of aluminum foil and packing them in a 1 -mm-thick cadmium box (20-mm internal diameter, $12-\mathrm{mm}$ internal height), which allows about 20 samples or standards to be irradiated together. Most irradiations for epithermal activation have been carried out in the JEEP-II reactor in Kjeller, Norway, in a position where the thermal neutron flux was about $1.5 \times 10^{13}$ neutrons $\mathrm{cm}^{-2} \mathrm{~s}^{-1}$, and the ratio of thermal to fast neutrons (as measured by a gold foil monitor) is 3.0. After irradiation, the aluminum foil packets must be opened, and the powdered samples transferred to clean polyethylene vials for counting. Because of the additional handling and the additional radiological hazard involved in manipulating highly radioactive powdered samples, epithermal activation cannot be considered as a routine method and is applied only for special studies requiring greater sensitivity for those elements whose determination is enhanced by epithermal activation.

\section{Standards}

A single multielement irradiation standard is used for the analysis of most rock samples in each laboratory. The standard used in the Reston laboratory was prepared by grinding obsidian (from Horse Mountain, Oregon) in an agate mortar to $<200$ mesh. For some trace elements, the intensity of the spectral lines of the indicator radionuclides have been enhanced by spiking the ground obsidian 
Table 2. Short-lived ( $t_{1 / 2}<3$ hours) indicator radionuclides [h, hours; $m$, minutes]

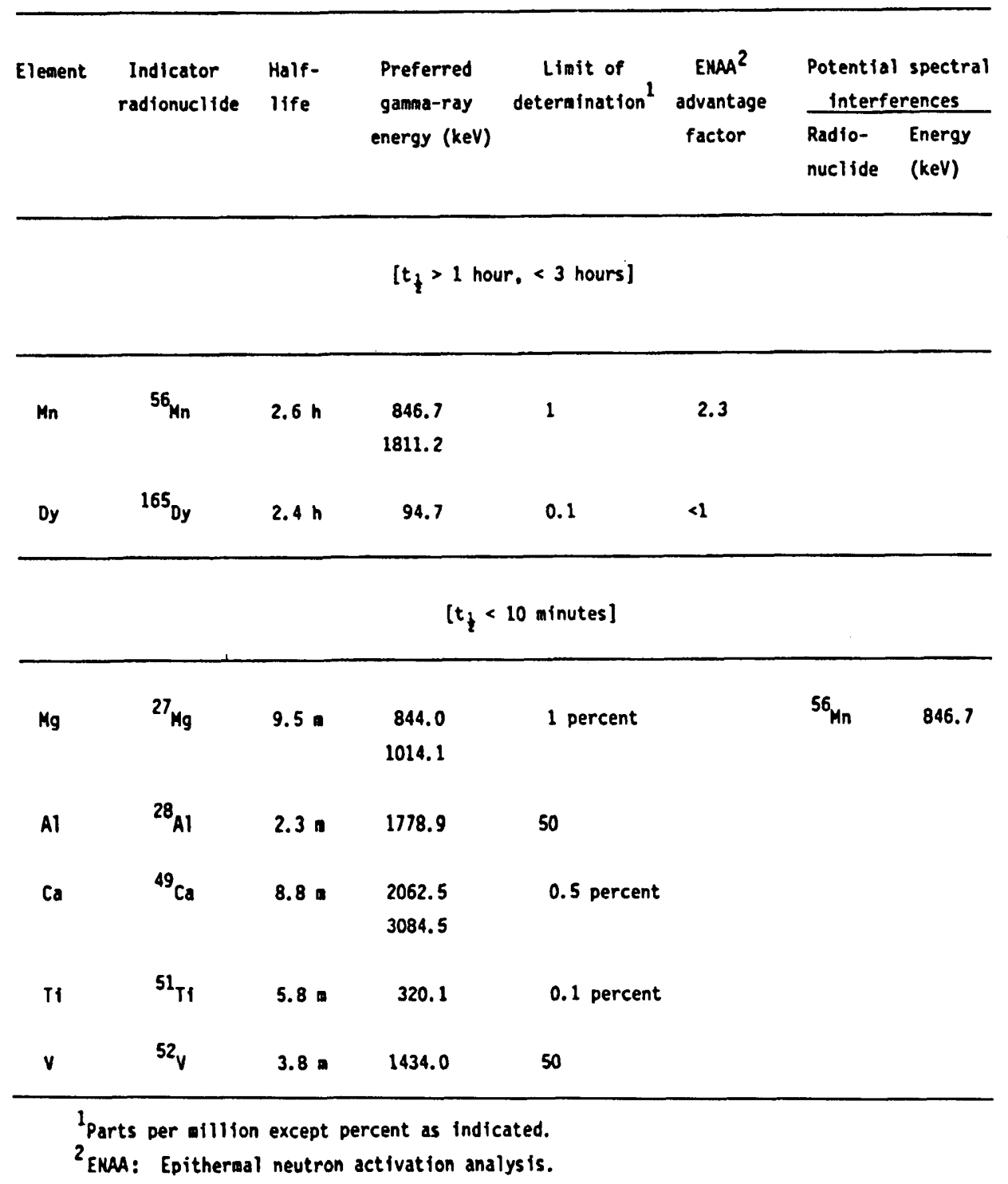

material with solutions of the elements of interest. The solutions were evaporated to dryness under a heat lamp with constant stirring; the material was further dried at $100^{\circ} \mathrm{C}$ overnight and reground to $<200 \mathrm{mesh}$. The obsidian standard (referred to as HMS-3) was calibrated by INAA using the five Geological Survey reference samples, basalt BCR-1, granite G-2, andesite AGV-1, granodiorite GSP-1, and basalt $W-1$. The initial values for the seven standard rocks were selected from the literature, primarily using a compilation prepared by Flanagan (1976). In general, data obtained by neutron activation and (or) by mass spectrometric isotope dilution analysis were used wherever possible. The Denver laboratory uses a standard (CQS-3) that was prepared in a similar manner by spiking high-purity quartz.

\section{Counting Facilities}

Following an irradiation in the TRIGA reactor, all samples are packaged within 2-dram polyethylene vials, which are used as transfer containers for the automatic sample changers used in conjunction with the highresolution coaxial $\mathrm{Ge}$ and LEPD detectors used for gamma-ray spectroscopy. For the normal suite of elements determined after an 8-h irradiation (table 1), a sequence of five to six counts on Ge and LEPD detectors over a 60-d decay period is used to maximize the number of elements determined and for highest precision and accuracy. [Separate single irradiation and counting sequences are used for the two short-lived element groups (table 2).] For routine analysis, all samples are counted 


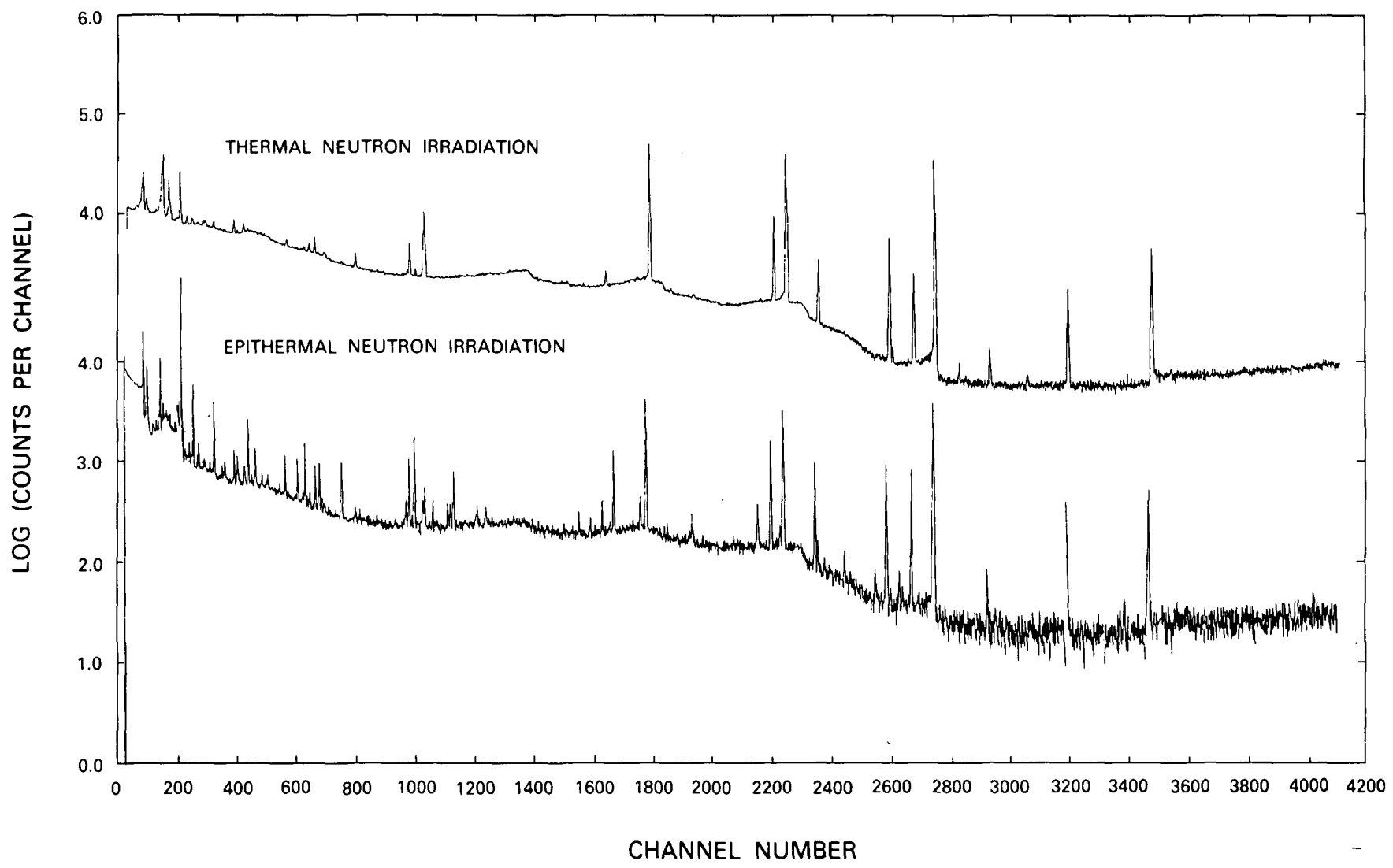

Figure 3. Germanium detector spectra of a neutron activated basalt sample irradiated with and without a cadmium cover (10 days decay).

for $1 \mathrm{~h}$, except for the final Ge and LEPD counts, which are $2 \mathrm{~h}$ each. The detectors are coupled to multichannel pulse height analyzers, which are capable of breaking the spectrum down into 4,096 energy increments, or channels. The analyzers automatically repeat a cycle of data acquisition, sample changing, and read out of the spectral data to disk storage. Following the acquisition of the spectral data, the data are transmitted (through a direct link or magnetic tape) to a VAX computer for analysis. The automatic sample changer used with each detector is mechanically similar to that described by Massoni and others (1973) except that, in the Denver laboratories, the changers now operate under pneumatic rather than solenoid control and that, in both laboratories, the electronic interfaces use entirely solid-state circuitry. The sample changer is a gravity feed device, which uses compressed air to eject a sample from the counting station after each count has been completed.

\section{Data Reduction}

Typical multichannel scaler information from a single Ge detector count in an INAA experiment contains 4,096 channels of data, and the data from an LEPD count are stored in 2,048 channels. Because the analysis of a single sample by INAA can involve several countings during a 2-mo decay period (typically three Ge detector counts and two LEPD counts), the data reduction process can involve the analysis of 16,384 channels of information (not including standard samples). For several years, the combined requests for INAA analysis to the Reston and Denver laboratories have been between 3,000 and 4,000 samples per year. It is obvious that such a flood of information can be processed only by means of a computer. The spectral information from LEPD and coaxial Ge counting is stored on computer-compatible magnetic tape and processed by using programs that have been developed for the analysis of gamma-ray spectra. The programs contain algorithms for smoothing the spectral data, searching out all peaks in the spectra, determining the areas of well-resolved single peaks and for resolving overlapping or complex peaks, and determining the accurate energies of the photopeaks (with corrections for zero and gain drift of the spectrometer). By using the data for specified peaks from elemental standard and sample spectra, the elemental concentrations are calculated with corrections for spectral interferences, decay, and neutron flux variations, as well as pulse pile-up effects in the detector-amplifier electronics. When a gamma-ray line 
has been specified for use in the analysis and has been observed in a standard sample but not the unknown, an upper limit to the concentration of the element in question is calculated based on an analysis of the statistical fluctuations in the background region at the expected peak location in the spectrum. Corrections for spectral interferences that are too poorly resolved to be recognized as multiplets in the peak search procedure are made when an interference-free line of the interfering radionuclide can be observed in the spectrum and used for the interference correction (as discussed in "Factors Affecting Accuracy"). Following the spectral analysis, additional software is used to average the results from multiple lines and multiple sample counts and to prepare a final report of analysis. If uranium has been determined (using the $2.35-\mathrm{d}^{239} \mathrm{~Np}$ ), then corrections for fission product interference in the determination of $\mathrm{Zr}, \mathrm{Mo}, \mathrm{La}, \mathrm{Ce}$, and $\mathrm{Nd}$ are calculated (as discussed in "Factors Affecting Accuracy").

Baedecker (1976) and Grossman and Baedecker (1986) published descriptions of the data processing scheme as developed for the Reston laboratory, as well as a series of papers that review the analysis of gamma-ray spectra (Baedecker, 1971, 1977, 1980). Data analysis in the Denver laboratory is carried out by using commercially available software (Nuclear Data Corporation) for spectral analysis, externally written software for the iterative least-squares analysis of complex peaks (SAMPO written by J. T. Routti, Lawrence Radiation Laboratory, Berkeley, California), and internally produced software for data management and report form generation.

\section{LIMITATIONS}

\section{Factors Affecting Precision}

1. Weighing errors. - Transcription errors are eliminated by coupling the laboratory balance to a computer, but operator errors could cause either a positive or negative bias in the results.

2. Nonreproducible positioning of the sample during counting.-This source of error has been tested in our laboratories and is generally negligible for $\mathrm{Ge}$ counting within the normal error limits, which are controlled by counting statistics. LEPD detectors are more sensitive to changes in counting geometry, and such errors may lead to a loss of precision of 1-2 percent for those elements that are determined exclusively by LEPD spectrometry.

3. Nonuniform distribution of the neutron flux across the sample irradiation position. - This has been tested and found to be negligible for the "lazy susan" facility at the Geological Survey reactor in Denver. Samples irradiated in the higher flux facility at the
National Bureau of Standards are subject to a 16percent variation in reactor flux along the irradiation container. This flux variation has been found to be constant over several years, and corrections can be applied during data processing.

4. Errors due to poor counting statistics and to photopeak baseline selection during photopeak integration.The Poisson counting error is evaluated during the data reduction process and reported along with the results of the analysis. Baedecker $(1971,1976)$ observed that the empirically evaluated precisions for the integration of several photopeaks in a typical $\mathrm{Ge}$ detector spectrum are well reflected by the expected error limits based on counting statistics.

5. Sampling errors. - In general, $600-\mathrm{mg}$ aliquots of powdered rock samples are processed in our laboratories. Rock samples are powdered to 100 mesh or finer by the sample preparation laboratory. The degree to which powdering a rock specimen will produce a homogeneous sample of this size is undoubtedly variable for major and accessory mineral phases and will vary depending on the abundance and grain size of accessory minerals that may act as hosts for the trace elements of interest. The sampling errors are best evaluated empirically for each set of similar samples by running replicate determinations.

Random errors generally can be reduced by running replicate analyses and averaging the results. Table 3 presents data that provides an evaluation of precision of our routine analytical procedure. Average values are listed for the concentrations of 23 elements in the reference samples BCR-1 and G-2 analyzed as controls in 20 separate irradiations between February 1981 and September 1982 relative to INAA standard HMS-2. Also listed are the standard deviation on a single determination based on the scatter of the results and the average estimated standard deviation based on counting statistics. We also have tested experimentally our precision within a single irradiation by analyzing six replicates each of both reference samples and observed that the relative sample standard deviation was within 1 percent of that expected based on counting statistics. The results presented in table 3 suggest that we lose roughly 1-3 percent between irradiations due to the additional factors other than counting statistics listed above. The poor precision for the determination of $\mathrm{Tb}$ and $\mathrm{Zr}$ is the result of problems associated with the determination of these elements due to spectral interferences (and fission product interference in the determination of $\mathrm{Zr}$ ), described below.

\section{Factors Affecting Accuracy}

1. Interfering nuclear reactions on other elements that yield 
Table 3. Precision of the determination of 23 elements in basalt BCR-1 and granite G-2 by instrumental neutron activation analysis

\begin{tabular}{|c|c|c|c|c|c|c|c|c|}
\hline \multirow{4}{*}{$\begin{array}{l}\text { Element } \\
\mathrm{Fe}\end{array}$} & \multicolumn{4}{|c|}{ BCR-1 } & \multicolumn{4}{|c|}{$\mathrm{G}-2$} \\
\hline & \multirow{2}{*}{ Consensus 1} & \multirow{3}{*}{$\begin{array}{c}\text { Average }^{2} \\
9.52\end{array}$} & \multirow{2}{*}{$\begin{array}{c}\text { percent } \\
\text { S.0. } \\
\text { sample } \\
\end{array}$} & \multirow{2}{*}{$\begin{array}{c}\text { percent } \\
\text { S. D. } \\
\text { count ing }\end{array}$} & Consensus ${ }^{1}$ & \multirow{3}{*}{$\frac{\text { Average }^{2}}{1.88}$} & \multicolumn{2}{|c|}{ percent percent } \\
\hline & & & & & & & $\begin{array}{c}\text { S.D. } \\
\text { sample } \\
\end{array}$ & $\begin{array}{c}\text { S.D. } \\
\text { counting }\end{array}$ \\
\hline & $9.38 \pm 0.22$ & & 2 & 1 & $1.87 \pm 0.07$ & & 3 & 1 \\
\hline Ba & $678 \pm 16$ & 690 & 3 & 3 & $1880 \pm 20$ & 1834 & 4 & 1 \\
\hline Co & $36.3 \pm 1.6$ & 35.9 & 2 & 1 & $4.6 \pm 0.4$ & 4.3 & 3 & 1 \\
\hline $\mathrm{Cr}$ & $16 \pm 4$ & 11.2 & 11 & 6 & $9 \pm 2$ & 7.0 & 8 & 4 \\
\hline Cs & $0.97 \pm 0.13$ & 1.0 & 11 & 7 & $1.33 \pm 0.14$ & 1.3 & 5. & 4 \\
\hline Hf & $4.9 \pm 0.3$ & 4.7 & 3 & 1 & $7.9 \pm 0.7$ & 7.7 & 4 & 1 \\
\hline Rb & $47.1 \pm 0.6$ & 53 & 8 & 6 & $170 \pm 3$ & 165 & 3 & 2 \\
\hline Ta & $0.79 \pm 0.09$ & 0.90 & 7 & 4 & $0.88 \pm 0.12$ & 0.91 & 5 & 3 \\
\hline Th & $6.0 \pm 0.6$ & 6.0 & 3 & 2 & $24.6 \pm 1.5$ & 24.1 & 3 & 1 \\
\hline U & $1.71 \pm 0.16$ & 1.6 & 10 & 9 & $2.04 \pm 0.17$ & 2.0 & 18 & 8 \\
\hline $2 n$ & $129 \pm 1$ & 135 & 6 & 2 & $85 \pm 7$ & 78 & 5 & 2 \\
\hline $2 r$ & $191 \pm 5$ & 210 & 38 & 16 & $300 \pm 30$ & 351 & 8 & 5 \\
\hline Sc & $32.8 \pm 1.7$ & 31.6 & 2 & 1 & $3.5 \pm 0.4$ & 3.29 & 2 & 1 \\
\hline La & $25.0 \pm 0.08$ & 26 & 3 & 1 & $86 \pm 5$ & 90 & 2 & 1 \\
\hline Ce & $53.7 \pm 0.8$ & 50 & 3 & 1 & $159 \pm 11$ & 153 & 3 & 1 \\
\hline Nd & $28.7 \pm 0.6$ & 31 & 9 & 7 & $53 \pm 8$ & 52 & 6 & 3 \\
\hline Sn & $6.58 \pm 0.17$ & 6.6 & 5 & 1 & $7.2 \pm 0.6$ & 7.4 & 5 & 1 \\
\hline Eu & $1.96 \pm 0.05$ & 1.79 & 3 & 1 & $1.41 \pm 0.12$ & 1.25 & 3 & 1 \\
\hline Gd & $6.68 \pm 0.13$ & 6.6 & 17 & 11 & $4.1 \pm 0.8$ & 5.1 & 14 & 6 \\
\hline Tb & $1.05 \pm 0.09$ & 1.06 & 19 & 4 & $0.48 \pm 0.07$ & 0.38 & 31 & 11 \\
\hline Tm & $0.59 \pm 0.04$ & 0.49 & 13 & 12 & $0.17 \pm 0.07$ & 0.15 & 21 & 14 \\
\hline$Y_{b}$ & $3.39 \pm 0.08$ & 3.4 & 3 & 2 & $0.78 \pm 0.14$ & 0.8 & 9 & 5 \\
\hline Lu & $0.51 \pm 0.03$ & 0.51 & 3 & 2 & $0.11 \pm 0.02$ & 0.10 & 8 & 5 \\
\hline
\end{tabular}

I Gladney and others (1983).

${ }_{3}^{2}$ Average of 20 analyses.

S. D. -standard deviation. 
interferences in thermal neutron activation are most often those due to radionuclides produced during the fission of ${ }^{235} \mathrm{U}$; for example, $\mathrm{Ce}$ is determined using the ${ }^{140} \mathrm{Ce}(\mathrm{n}, \gamma){ }^{141} \mathrm{Ce}$ reaction. ${ }^{141} \mathrm{Ce}$ also is produced by thermal neutron-induced fission of ${ }^{235} \mathrm{U}$. The calculated interference [defined as the ratio of the ${ }^{141} \mathrm{Ce}$ activity per microgram of $U$ produced in fission to the activity per microgram of Ce produced by the $(n, \gamma)$ reaction of ${ }^{140} \mathrm{Ce}$ ] is 0.27 . Therefore, a concentration of $2 \mathrm{ppm} U$ in a rock sample would contribute $0.54 \mathrm{ppm}$ to the apparent $\mathrm{Ce}$ concentration. Theoretical and empirical correction factors for fission product interferences are tabulated in table 4.

The calculation of the fission product interference for $\mathrm{La}$ is complicated by the fact that the fission product ${ }^{140} \mathrm{Ba}$ has a longer half-life $(12.7 \mathrm{~d})$ than its daughter product, the indicator radionuclide ${ }^{140} \mathrm{La}$. The extent of the interference is controlled by the growth and the decay of ${ }^{140} \mathrm{La}$ following the irradiation and, therefore, the decay interval between the end of the irradiation and the time of counting. The tabulated values for La are, therefore, for a decay time of $10 \mathrm{~d}$ following irradiation, which is the normal decay time for the determination of La. Fission product interferences can be reduced by epithermal irradiation.

2. Gamma-ray spectral interferences. - These result when a gamma-ray line from a second radionuclide overlaps a gamma-ray line of the radionuclide of interest, and the two lines are not resolvable with the gamma-ray spectrometer employed or by computer-assisted data reduction; for example, ${ }^{65} \mathrm{Zn}$ has a single line at $1115.4 \mathrm{keV}$ that is subject to interference from the $1115.1-\mathrm{keV}$ line of ${ }^{160} \mathrm{~Tb}$. The relative intensity of the $1115.1-\mathrm{keV}$ line to other interference-free lines (the 879.4- or the $1178.1-\mathrm{keV}$ line) is used to make a correction to the measured intensity of the ${ }^{65} \mathrm{Zn}$ line. A similar problem arises in the determination of $\mathrm{Ba}$ using ${ }^{131} \mathrm{Ba}\left(\mathrm{t}_{\mathrm{y} / 2}=11.5 \mathrm{~d}\right)$, where the $496.4-\mathrm{keV}$ photopeak has an interference due to the $497.1-\mathrm{keV}$ photopeak of fission product ${ }^{103} \mathrm{Ru}$. Because no satisfactory interference-free line of ${ }^{103} \mathrm{Ru}$ is available for applying a correction, the Ba results based on the $497-\mathrm{keV}$ line are checked against those from

Table 4. Interferences in instrumental neutron activation analysis due to neutron-induced fission of uranium-235

[In micrograms of the elements (apparent) per microgram of uranium]

\begin{tabular}{|c|c|c|c|c|c|c|}
\hline \multirow{2}{*}{$\begin{array}{l}\text { Isotope } \\
{ }^{95} \mathrm{Zr}\end{array}$} & \multirow{2}{*}{$\begin{array}{c}\begin{array}{c}\text { Fission } \\
\text { yield }\end{array} \\
6.5\end{array}$} & \multirow{2}{*}{$\begin{array}{c}(n, \gamma) \text { cross } \\
\text { section } \\
\text { (barns) } \\
0.08\end{array}$} & \multirow{2}{*}{$\begin{array}{l}\begin{array}{l}\text { Interference } \\
\text { (calculated) }\end{array} \\
7.5\end{array}$} & \multicolumn{3}{|c|}{$\begin{array}{l}\text { Interference } \\
\text { (experimental) }\end{array}$} \\
\hline & & & & 11.5 & \pm & 0.3 \\
\hline $99_{\text {Mo }}$ & 6.1 & 0.51 & 0.85 & 1.0 & \pm & 0.1 \\
\hline${ }^{140} \mathrm{La}$ & 6.3 & 8.9 & $0.092^{a}$ & 0.092 & \pm & $0.005^{\mathrm{a}}$ \\
\hline${ }^{141} \mathrm{Ce}$ & 5.9 & 0.6 & 0.27 & 0.29 & \pm & 0.01 \\
\hline${ }^{147}$ Nd & 2.26 & 2 & 0.17 & 0.22 & \pm & 0.01 \\
\hline${ }^{153} \mathrm{sm}$ & 0.16 & 210 & 0.00008 & & & \\
\hline
\end{tabular}

${ }^{1}$ Cross section for fission of ${ }^{235} U-580$ barns.

2Error estimates are 70 percent confidence 1 fraits on the aean of four deterainations.

a d following irradiation. 
the less intense $373-\mathrm{keV}$ line and against the 124$\mathrm{keV}$ line in the LEPD spectra (which must be resolved from a ${ }^{152} \mathrm{Eu}$ interference).

If a spectral interference in either the sample or the standard is unknown or if no correction for interference is made, then a positive or negative error, respectively, would result. Potential spectral interferences for which corrections may have to be made are listed in table 1.

3. Self shielding. - This is an attenuation of the neutron flux by the sample and is generally negligible in most silicate matrices. The problem becomes important when the matrix contains a high concentration of a nuclide (or nuclides) with a large thermal neutron-capture cross section. Several rareearth nuclides have large cross sections, and self shielding could contribute to a substantial error in the analysis of rare-earth minerals. The effects of self shielding can be minimized by reducing the size of the sample taken for analysis; for example, a rare-earth mineral might be ground and mixed with a measured quantity of high-purity quartz to prepare a sample with a lower average cross section for neutron absorption.

4. Dead-time errors. - These occur during gamma-ray counting when the activity of the sample differs considerably from that of the standard. Systematic errors arise because of different relative counting losses. These losses can result either from the summing (or "pile-up") of pulses within the detector-amplifier system or from pulses that are not processed by the analog to digital converter (ADC) while the ADC is busy processing prior pulses. At present, corrections for both types of losses generally are made by using special circuitry that is built into amplifiers and analog-to-digital converters for gamma-ray spectroscopy. When amplifiers with pulse pile-up-correcting circuity are not available, mathematical corrections are made during the data reduction process based on empirically measured pulse pile-up-resolving times or by imposing a peak on the gamma-ray spectrum from a fixed-rate pulse generator at the detector preamplifier. Failure to correct properly for such losses in either sample or standards can lead to systematic negative or positive errors, respectively. Differences in dead time can be minimized by using a counting geometry that reduces the dead time of the spectrometer. Corrections for dead-time losses become more critical in the case of short-lived $\left(t_{v,}<10 \mathrm{~min}\right.$ ) activities (for example, in the determination of $\mathrm{Al}$, $\mathrm{V}, \mathrm{Mg}, \mathrm{Ti}, \mathrm{Ca}$ ) where the dead time and the relative intensity of the spectral lines change during the counting period.

5. Differences in the powder density - These differences between samples and standards can cause a systematic error because of the resulting differences in counting geometry (and, therefore, counting efficiency). Where such an error is suspected, it may be desirable to use one or more elements in the sample as an internal standard, if data on those elements in the same sample are available from some independent method. Small mineral separates generally are analyzed by comparison with identically prepared standards of similar composition or by dilution of the sample with high-purity quartz powder.

6. Errors in the preparation or calibration of standards. These are best evaluated by the analysis of wellcharacterized control samples that have been analyzed by independent methods and (or) laboratories.

\section{Problems With the Determination of Individual Elements}

Some of the problems associated with the determination of specific elements are described as follows.

Zinc

The $1115-\mathrm{keV}$ photopeak of ${ }^{65} \mathrm{Zn}$ falls on the low-energy tail from the often intense $1120-\mathrm{keV}$ photopeak of ${ }^{46} \mathrm{Sc}$, which complicates the evaluation of the base area for the photopeak. Normally, it is treated as a triplet along with the $1112-\mathrm{keV}$ photopeak of ${ }^{152} \mathrm{Eu}$ by the computer. Also, there is a small spectral interference from ${ }^{160} \mathrm{~Tb}$ that is less than 2 percent in standard sample G-2.

\section{Zirconium}

Zirconium is difficult to determine by INAA, due to spectral interferences from ${ }^{152} \mathrm{Eu},{ }^{154} \mathrm{Eu}$, or ${ }^{160} \mathrm{~Tb}$ that overlap the lines from ${ }^{95} \mathrm{Zr}$ and its daughter product, ${ }^{95} \mathrm{Nb}\left(\mathrm{t}_{1 / 2}=35.1 \mathrm{~d}\right)$. In $\mathrm{G}-2$, the correction on the ${ }^{95} \mathrm{Nb}$ $765.8-\mathrm{keV}$ line is 9 percent. It is also necessary to correct for the fission product interference based on the measured $\mathrm{U}$ content, which, for $\mathrm{G}-2$, amounts to 8 percent. For these reasons, the determination of $\mathrm{Zr}$ is semiquantitative in many samples.

\section{Barium}

Problems associated with the determination of $\mathrm{Ba}$ using the $496.3-\mathrm{keV}$ line from ${ }^{131} \mathrm{Ba}$ due to spectral interference from fission product ${ }^{103} \mathrm{Ru}$ have been discussed above. An estimate of the interference calculated 
from fundamental parameters is $2.9 \times \mathrm{e}^{0.0402 \mathrm{t}}$ (apparent parts per million Ba per parts per million $U$ ), where $t$ is the time after irradiation in days. Alternative interferencefree, but less intense, ${ }^{131} \mathrm{Ba}$ lines provide poorer sensitivity, and satisfactory Ba results generally can be obtained only at the 100-ppm level and above.

\section{Gadolinium}

Gd is determined by LEPD counting at least 1 mo following the irradiation. The analysis is based on the measurement of a weak $97.5-\mathrm{keV}$ line, which must be resolved by computer analysis from a neighboring 98.4$\mathrm{keV}$ U-K X-ray from the decay of ${ }^{233} \mathrm{~Pa}$. Because of the poor sensitivity and possible errors due to geometry problems on small detectors, the precision for $\mathrm{Gd}$ is generally no better than \pm 10 percent.

\section{Terbium}

The determination of $\mathrm{Tb}$ is made difficult by spectral interferences. The most intense line in the germanium detector spectra at 298.6 occasionally suffers from interferences from the $299.9-\mathrm{keV}$ line of ${ }^{233} \mathrm{~Pa}$. The computer must first check to see if both lines have been detected and resolved by the multiplet analysis algorithm, and, if not, a correction must be applied for the interference. The 879.4-keV line also is observed in most spectra as a weak line on the low-energy tail of the generally intense ${ }^{46} \mathrm{Sc} 889.3-\mathrm{keV}$ line, which renders the base area difficult to evaluate. The $965.8-\mathrm{keV}$ line may suffer from interference from ${ }^{152} \mathrm{Eu}$, and, although the line at 1177.9$\mathrm{keV}$ is free from interferences, it has much poorer sensitivity.

\section{Thulium}

$\mathrm{Tm}$ is determined by LEPD counting at least $1 \mathrm{mo}$ following the irradiation. The analysis is based on the measurement of a weak $84.3-\mathrm{keV}$ line, which must be resolved by computer analysis from a neighboring 84.7$\mathrm{keV}$ line from the decay of ${ }^{182} \mathrm{Ta}$, or an interference correction must be applied when the peaks cannot be resolved.

\section{Ytterbium}

Photopeaks from ${ }^{175} \mathrm{Yb}\left(\mathrm{t}_{1 / 2}=101 \mathrm{hr}\right)$ and ${ }^{169}\left(\mathrm{t}_{1 / 3}=\right.$ $32 \mathrm{~d})$ normally are detected in most Ge detector spectra of activated rock samples. Most phutopeaks from both nuclides suffer from potential spectral interferences, although $\mathrm{Yb}$ is generally well determined using the 396.1$\mathrm{keV}$ line of the shorter lived isotope. Potential minor interferences are possible from ${ }^{147} \mathrm{Nd}$ and ${ }^{233} \mathrm{~Pa}$.

\section{SUMMARY}

Instrumental neutron activation analysis is a powerful tool for the analysis of geological samples. The technique is capable of yielding data on over 30 elements in rock samples. It is amenable to a high degree of automation by using procedures for automatic data acquisition and data reduction. A single analysis may take 2 or 3 mo to complete because optimum results are achieved by counting the samples at various times during the decay of the radioactivities induced in the sample. A summary of the general features of the INAA procedure as routinely applied to the analysis of geological materials is presented in table 5 .

\section{REFERENCES CITED}

Baedecker, P. A., 1971, Digital methods of photopeak integration in activation analysis. Analytical Chemistry, v. 43, p. 405-410.

1976, SPECTRA: Computer reduction of gamma-ray spectroscopic data for neutron activation analysis, in Taylor, R. E., ed., Advances in Obsidian Glass Studies: Park Ridge, New Jersey, Noyes Press, p. 334-349.

1977, The precision and accuracy of iterative and noniterative methods of photopeak integration in activation analysis, with particular reference to the analysis of multiplets: Journal of Radioanalytical Chemistry, v. 39, p. 239-254.

-1980, Comparisons of peak-search and photopeakintegration methods in the computer analysis of gammaray spectra: International Conference in Nuclear Methods in Environmental and Energy Research, 4th, Columbia, Missouri, 1980, Proceedings, CONF-800433, U.S. Dept. of Energy, p. 15-24.

Baedecker, P. A., Rowe, J. J., and Steinnes, E., 1977, Application of epithermal neutron activation in multielement analysis of silicate rocks employing both coaxial $\mathrm{Ge}(\mathrm{Li})$ and low energy photon detector systems: Journal of Radioanalytical Chemistry, v. 40 , p. $115-146$.

Brunfelt, A. O., and Steinnes, E., 1969, Instrumental activation analysis of silicate rocks with epithermal neutrons. Analytical Chimica Acta v. 48, p. 13-24.

Flanagan, F. J., ed., 1976, Description and analysis of eight new USGS rock standards: U.S. Geological Survey Professional Paper 840.

Gladney, E. S., Burns, C. E., and Roelandts, I., 1983, 1982 Compilation of elemental concentrations in eleven United States Geological Survey rock standards: Geostandards Newsletter, v. 7, p. 3-226.

Gordon, G. E., Randle, K., Goles, G. G., Corliss, J. B., Beeson, M. H., and Oxley, S. S., 1968, Instrumental activation 


\begin{tabular}{|c|c|}
\hline Elenents measured: & $\begin{array}{l}\text { Na, Sc, Cr, Fe, Co, Zn, Rb, Zr, Sb, Cs, Ba, La, Ce, Nd, } \\
\text { Sa, Eu, Gd, Tb, Tn, Yb, Lu, Hf, Ta, Th, U } \\
\text { (Mg, Al, K, Ca, Ti, V, Mn, Sr, Mo, Oy, W, Au--with } \\
\text { special irradiation and counting procedures). }\end{array}$ \\
\hline Suftable matrices: & $\begin{array}{l}\text { Most silicate whole rocks, soils, and some nineral } \\
\text { phases and low grade ores. }\end{array}$ \\
\hline Matrix limitations: & $\begin{array}{l}\text { High-grade metal ores; some pyrites; and some heavy metal } \\
\text { mineral phases. Rare-earth-element determinations } \\
\text { (less than approximately } 5 \text { times chondrites) in ultra- } \\
\text { mafic rocks. }\end{array}$ \\
\hline Other limitations: & $\begin{array}{l}\text { Decreased sensitivity and confidence for certain elements } \\
\text { if uraniun content is greater than } 500 \mathrm{ppm} \text { or if rare- } \\
\text { earth abundances are greater than } 10,000 \text { times } \\
\text { chondrite. Common rock detection } 1 \text { imits are given in } \\
\text { tables } 1 \text { and } 2 \text {. }\end{array}$ \\
\hline Sample requirements: & $\begin{array}{l}\text { One-half to one gran of powdered or finely granulated } \\
\text { material. Special arrangements, however, can accom- } \\
\text { modate lesser amounts of separated mineral phases (as } \\
\text { small as } 50-100 \mathrm{mg} \text { ). }\end{array}$ \\
\hline Standardization: & $\begin{array}{l}\text { Normalized spectral comparison to synthetic multielement } \\
\text { standard prepared by doping silicate powder with ele- } \\
\text { mental solutions of known concentrations. Standard } \\
\text { values checked against Geological Survey standard } \\
\text { rocks. }\end{array}$ \\
\hline Quality control: & $\begin{array}{l}\text { At least one Geological Survey standard rock is assayed } \\
\text { with each analys is set to monitor interexperiment } \\
\text { variations and intraexperiment accuracy. }\end{array}$ \\
\hline Throughput: & $\begin{array}{l}\text { Thirty-three to thirty-six samples are included in each } \\
\text { experiment (irradiation set). Turn around time, start } \\
\text { to data report, is typically 10-12 weeks for each } \\
\text { experiment set due to the long decay tiae before final } \\
\text { spectral measurement. }\end{array}$ \\
\hline
\end{tabular}

analysis of standard rocks with high-resolution X-ray detectors: Geochemica et Cosmochimica Acta v. 32, p. 369-396.

Grossman, J. N., and Baedecker, P. A., 1986, Computer graphics for quality control in the INAA of geological samples: Seventh International Conference on Modern Trends in Activation Analysis, Copenhagen, Denmark, 1986, Proceedings, p. 571-578.

Hertogen, J., and Gijbels, R., 1971, Instrumental neutron activation analysis of rocks with a low-energy photon detector: Analytica Chimica Acta v. 56, p. 61-82.

Jacobs, J. W., Korotev, R. L., Blanchard, D. P., and Haskin, L. A., 1977, A well tested procedure for instrumental neutronactivation analysis of silicate rocks and minerals: Journal of Radioanalytical Chemistry, v. 40, p. 93-114.

Laul, J. C., 1979, Neutron activation analysis of geological materials: Atomic Energy Review, v. 17, p. 603-695.

Massoni, C. J., Fones, R. V., and Simon, F. O., 1973, A pneumatic sample changer for gamma-ray spectroscopy: Reviews of Scientific Instruments, v. 44, p. 1350-1352. 
CHAPTER I

Determination of Uranium and Thorium by Delayed Neutron Counting

By D. M. MCKOWN and H. T. MILLARD, JR.

U.S. GEOLOGICAL SURVEY BULLETIN 1770

Methods for Geochemical Analysis 



\title{
CONTENTS
}

\author{
Abstract I1 \\ Introduction I1 \\ Delayed neutrons I1 \\ Principles of delayed neutron analysis $\mathbf{I 1}$ \\ Delayed neutron analysis facilities $\mathbf{I 3}$ \\ Analytical procedures $\mathbf{1 5}$ \\ Experiment organization $\mathbf{1 5}$ \\ Sample preparation $\mathbf{I 5}$ \\ Irradiation counting $\mathbf{I 5}$ \\ Data reduction I6 \\ Discussion $\quad$ 7 \\ Detection limits $\quad$ 7 \\ Precision and accuracy 17 \\ Accuracy $\quad$ I7 \\ Interferences and limitations $\mathbf{I 8}$ \\ Summary III \\ References cited III
}

\section{FIGURES}

1. Automated delayed neutron system I4

2. Average coefficient of variation for each decade interval of concentration plotted against the average concentration for the decade 18

\section{TABLES}

1. Decay properties of delayed neutron emitters resulting from thermal and fast-neutron-induced fission of uranium and thorium $\mathbf{I}$

2. Analytical parameters for a complete cycle of two irradiations and appropriate counting sequence I6

3. Uranium and thorium values obtained by replicate delayed neutron analyses compared to published consensus values I9

4. Comparison of single-analysis delayed neutron data with reference values from a set of reference samples analyzed during a normal run $\mathbf{I 1 0}$

5. Summary of analytical features of delayed neutron analysis of geologic materials III 



\title{
Determination of Uranium and Thorium by Delayed Neutron Counting
}

\author{
By D. M. McKown and H. T. Millard, Jr.
}

\begin{abstract}
Delayed neutron counting is a nuclear activation analysis method for measuring uranium and thorium in a complex sample matrix without chemical processing. The delayed neutron counting method is generally applicable for routine analysis of a wide variety of geological materials, including most common silicate rocks, soils, and some moderately mineralized materials that exhibit a thorium to uranium ratio greater than 3 . For these suitable types of materials, the analysis of a 10-gram sample aliquot exhibits detection limits of about 0.1 part per million for uranium and 1 part per million for thorium. Analytical precisions of about \pm 5 percent for uranium and \pm 10 percent for thorium may be achieved for the measurement of uranium and thorium concentrations greater than about 1 part per million and 10 parts per million, respectively. The analysis of uranium or thorium ores may exhibit decreased sensitivity and confidence for thorium if the thorium to uranium ratio is less than 3 and, similarly, for uranium if the ratio is greater than 50 . Samples that are highly anomalous in fluorine, beryllium, lithium, boron, cadmium, or gadolinium are generally not suitable for delayed neutron counting analysis.
\end{abstract}

\section{INTRODUCTION}

Delayed neutron counting (DN) is a rapid, instrumental, nuclear analysis method for measuring uranium and thorium in geologic samples. The DN procedure employs neutron irradiation of a sample to induce nuclear transformation of certain elements into radioactive nuclides. Following the irradiation, specific radiations emitted from these radioactive products are measured as an indicator of parent-element abundance. In these general aspects, DN analysis is conceptually similar to the instrumental neutron activation analysis (INAA) procedures detailed in Chapter H. However, because the DN method differs significantly in principle as well as in operational and application considerations, it is discussed separately in this section.

\section{Delayed Neutrons}

Reactor neutron irradiation of most stable elements yields neutron-capture products that are radioactive iso- topes of their parent elements. These products subsequently decay by emitting characteristic beta and gamma radiation. These processes provide a basis for neutron activation analysis techniques employing gamma-ray spectroscopy.

In contrast to these more common activation-decay processes, a few heavy-element nuclei, specifically those of $\mathrm{Th}, \mathrm{U}$, and the transuranium elements, capture a neutron to produce a nucleus so energetically unstable that it instantaneously fissions into two lighter element nuclei with release of one to several excess neutrons per fission event. These neutrons are called prompt fission neutrons. The two primary fission fragments, which may be isotopes of various elements ranging from zinc to terbium, are radioactive and usually decay by a delayed series of successive beta emissions to eventually form stable isotopes. A relatively small fraction of the fission products, however, decay by beta emission accompanied by release of a "delayed neutron" before further successive beta decay can occur.

As many as 50 individual delayed neutron emitters, each with a characteristic half-life in the range of about 0.2-56 s, probably exist among the total gross fission products (MacMurdo and Bowman, 1977). It generally is agreed, however, that an optimum least-squares fit to experimental neutron decay data, regardless of parent isotope, is obtained using only six half-life groups. The half-life and delayed neutron yield for each of these groups, as summarized by Laul (1979), are listed in table 1 for the fission of ${ }^{235} \mathrm{U},{ }^{238} \mathrm{U}$, and ${ }^{232} \mathrm{Th}$.

\section{Principles of Delayed Neutron Analysis}

Echo and Turk (1957) demonstrated that neutron irradiation followed by DN counting provided a sensitive, quantitative measure of the amount of a fissionable isotope in complex sample matrices. Other early development and evaluation of the delayed neutron analysis method have been reported in papers by Amiel (1962), Dyer and others (1962), Gale (1967), and Brownlee 
Table 1. Decay properties of delayed neutron emitters resulting from thermal and fast-neutron-induced fission of uranium and thorium [From Laul, 1979]

\begin{tabular}{|c|c|c|c|c|c|c|}
\hline \multirow[b]{2}{*}{ Group 1} & \multicolumn{2}{|c|}{ U-235 (thermal) } & \multicolumn{2}{|c|}{$U-238$ (fast) } & \multicolumn{2}{|c|}{ Th-232 (fast) } \\
\hline & $\begin{array}{c}\text { Half-life } \\
\text { (s) }\end{array}$ & $\begin{array}{l}\text { Yield }^{2} \\
\text { percent }\end{array}$ & $\begin{array}{l}\text { Half-life } \\
\text { (s) }\end{array}$ & $\begin{array}{l}\text { Yield } \\
\text { percent }\end{array}$ & $\begin{array}{c}\text { Half-life } \\
\text { (s) }\end{array}$ & $\begin{array}{l}\text { Yield } \\
\text { percent }\end{array}$ \\
\hline 1 & 55.72 & 0.052 & 52.38 & 0.054 & 56.03 & 0.169 \\
\hline 2 & 22.72 & 0.346 & 21.58 & 0.564 & 20.75 & 0.744 \\
\hline 3 & 6.22 & 0.310 & 5.00 & 0.667 & 5.74 & 0.769 \\
\hline 4 & 2.30 & 0.624 & 1.93 & 1.599 & 2.16 & 2.212 \\
\hline 5 & 0.61 & 0.182 & 0.49 & 0.927 & 0.57 & 0.853 \\
\hline 6 & 0.23 & 0.066 & 0.17 & 0.309 & 0.21 & 0.213 \\
\hline SUM & & 1.58 & & 4.12 & & 4.96 \\
\hline
\end{tabular}

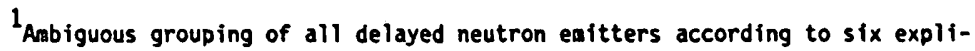
cit half-lives inferred from a least-squares fit to observed neutron-decay-rate data.

${ }^{2}$ Cumulative fission yield for corresponding half-life groups, expressed as a percentage of the total number of fissions.
}

(1968). Since this early development, the DN method has evolved in numerous activation analysis laboratories as a fast instrumental method for measurement of uranium and thorium in geological materials; for example, Rosenberg and others (1977), Millard (1976), and Gladney and others (1980).

Delayed neutrons from an irradiated sample can be detected and counted quantitatively with reasonably good efficiency and practically without interference from gamma-ray emitters. Without extremely elaborate detector systems, however, individual neutrons cannot be resolved based on their energy characteristics. Furthermore, as illustrated by data in table 1 , the delayed neutron activities from all fissionable isotopes exhibit essentially the same decay rate (half-lives for the various groups). The differences that may exist in the group half-lives are sufficiently small as to preclude their use in the resolution of the decay curve from a sample containing more than one fissionable isotope. Brownlee (1968) demonstrated limited success in the identification of component fissionable species in a mixture based on differences in half-life group abundances (table 1), but the equipment and methods employed in this approach are extremely complicated and not very suitable for routine applications.

In a practical sense, then, the measurement of delayed neutrons is limited to gross neutron counting. Consequently, the DN analysis method suffers from the disadvantage that no direct identification of individual fissionable species can be made solely from counting data. However, in certain cases where the individual fissionable components are qualitatively known and few in number, selective measurement may be derived indirectly from relative differences in their neutron-induced fission characteristics. 
These criteria are favorable for the application of $\mathrm{DN}$ as an elemental analysis method for the determination of $U$ and $T h$ in a wide variety of geological materials. The only fissionable isotopes found among the naturally occurring elements are those of uranium and thorium, specifically ${ }^{235} \mathrm{U},{ }^{238} \mathrm{U}$, and ${ }^{232} \mathrm{Th}$, which exhibit favorable differences in their cross sections (probability) for fissionactivation as a function of bombarding neutron energy. Thorium-232 and ${ }^{238} \mathrm{U}$ undergo fission only with highenergy (fast) neutrons, whereas ${ }^{235} U$ undergoes fission with high- and low-energy (thermal) neutrons but exhibits a relatively larger cross section for thermal neutron fission. Thus, if a composite sample is activated with thermal neutrons, then only delayed neutrons from uranium $\left({ }^{235} \mathrm{U}\right)$ are measured. If the sample is reirradiated with fast neutrons, then the three species undergo fission, but, having measured uranium by thermal activation and knowing the natural ${ }^{238} \mathrm{U}-{ }^{235} \mathrm{U}$ isotopic ratio, the thorium contribution may be obtained by difference.

In practice, it is not possible to obtain perfectly discriminated fast or thermal irradiations within the energy continuum flux of a nuclear reactor. However, a reasonable degree of discrimination is obtained by utilizing an unperturbed (bare) irradiation position located within the thermalizing material of a reactor where thermal neutrons are most prevalent and a second position, usually within the reactor core, that is surrounded by cadmium, which preferentially absorbs a large fraction of the thermal neutrons. Under typical bare terminus conditions, the fission contribution of uranium is about $350-400$ times that of an equal amount of thorium. For $\mathrm{Cd}$ shielded irradiations, the relative activity due to thorium is maximized although the uranium contribution is still about six times that of an equal amount of thorium. Even under optimum conditions, each element represents a mutual interference to the other. Normally, the thorium content causes only a minor correction for the measurement of uranium, but the uranium content may cause a major correction for the measurement of thorium. However, if the sensitivity for each element is measured individually for each irradiation-counting condition, then an iterative algorithm may be applied to the gross neutroncounting data for a composite sample to resolve individual contributions and compute the concentrations of uranium and thorium.

The specificity of DN analysis for fissionable elements results from the fact that very few nonfission interfering reactions are present. The only direct activation interferences are the following:

$$
\begin{aligned}
& { }^{17} \mathrm{O}(\mathrm{n}, \mathrm{p}){ }^{17} \mathrm{~N} \rightarrow{ }^{16} \mathrm{O}+\mathrm{n} \text { (delayed, half-life }=4.2 \mathrm{~s} \text { ) } \\
& \text { and } \\
& \left.{ }^{9} \mathrm{Be}(\mathrm{n}, \mathrm{p}){ }^{9} \mathrm{Li} \rightarrow{ }^{8} \mathrm{Be}+\mathrm{n} \text { (delayed, half-life }=0.2 \mathrm{~s}\right),
\end{aligned}
$$

where $(n, p)$ represents a nuclear reaction of the target nuclide and a neutron to produce the product nuclide and a proton. Interference from $\mathrm{Be}$ is generally negligible because of its low abundance and the short half-life of its delayed neutron product. Oxygen is present in major abundance, but the interference from oxygen is small due to a very small activation cross section and substantial decay before counting. Suitable corrections for oxygen interference can be made.

Delayed neutron counting provides a basis for relatively rapid, instrumental analysis of $U$ and Th. A thorough and precise DN analysis, however, requires a rather rigorous experimental procedure-sequential measurements of elemental standards and samples, precise control or monitoring of irradiation-decay-counting parameters, and rather extensive data reduction procedures. To accomplish this rigorism and to maintain practical expediency for routine applications, it is most desirable to employ an automated DN facility that integrates the irradiation termini, transfer systems, and counters with a computer that provides experiment control and data handling.

\section{Delayed Neutron Analysis Facilities}

The U.S. Geological Survey has supported the operation of a delayed neutron analysis facility since 1971 . In addition to analyzing tens of thousands of samples during this time, numerous studies related to the development and evaluation of DN methodology have been conducted. As a result, the delayed neutron analysis equipment has evolved from a manually operated system to a highly automated facility that embodies a high degree of practical expediency for large-scale routine utilization, yet allows precise control of irradiation parameters and extensive data acquisition necessary to yield reliable results.

The delayed neutron analysis facility currently maintained at the Geological Survey TRIGA reactor consists of four simultaneously operating irradiation-transfercounting systems. Figure 1 schematically represents the architecture of the DN facility with only one of the four transfer systems shown. The facility consists basically of the following integral, interfaced units:

1. Individual pneumatic transfer tubes (P-tubes) between the irradiation termini in the reactor and the neutron-counting assemblies,

2. A controller-timer-display (CTD) microprocessor that simultaneously controls the operation of all P-tubes and monitors the operation of each P-tube individually, and

3. A minicomputer-interface unit that provides programmable control for sequencing samples, collecting and recording data, and subsequent data reduction. 


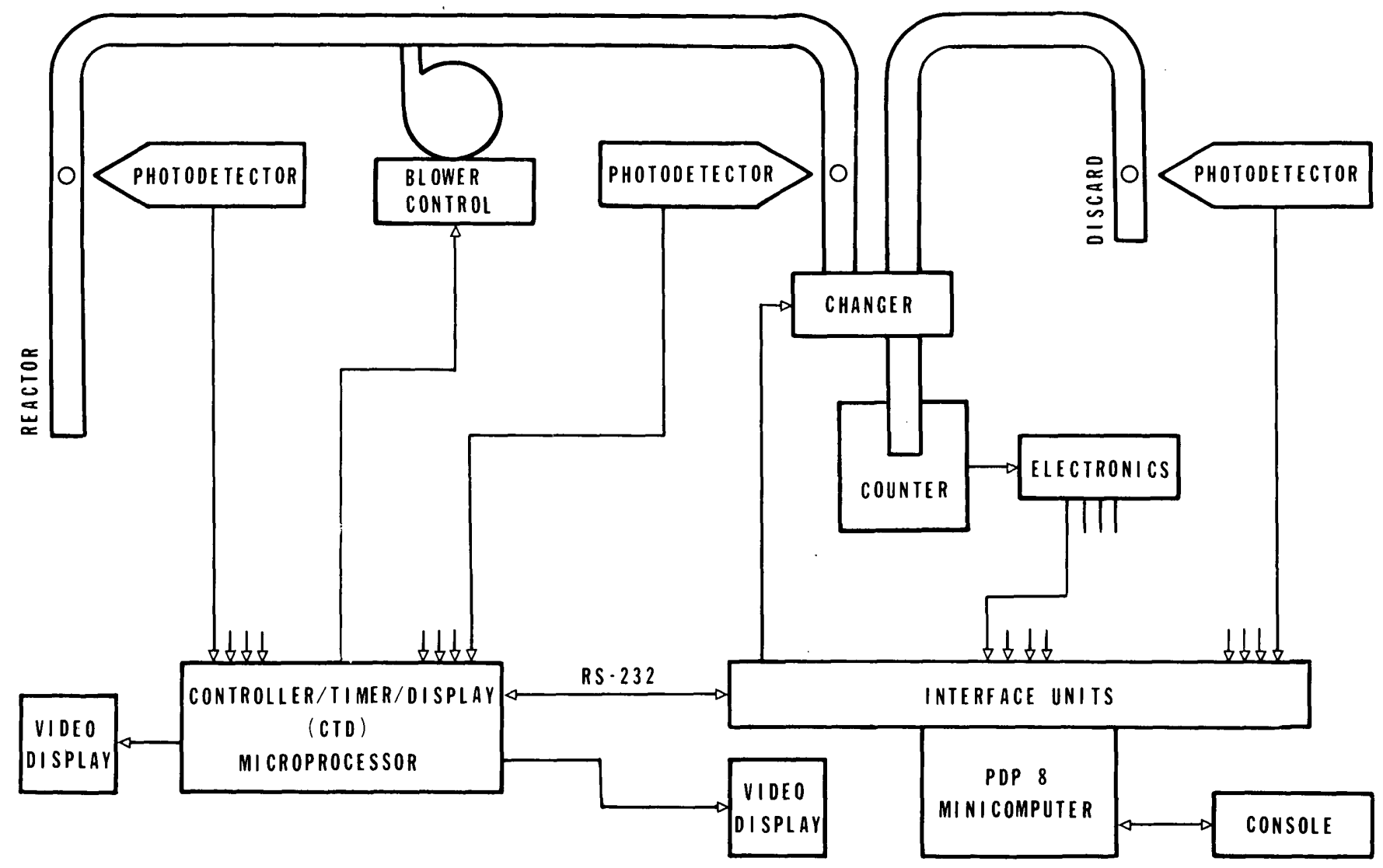

Figure 1. Automated delayed neutron system.

Two of the P-tubes terminate in the reactor at cadmium-lined, in-core positions, and the other two terminate at bare terminus positions outside the graphite reflector. With the reactor operating at $1 \mathrm{MW}$, the unperturbed neutron flux is about $6 \times 10^{12}$ neutrons $\mathrm{cm}^{-2} \mathrm{~s}^{-1}$ at the cadmium-lined positions and approximately $1 \times 10^{12}$ neutrons $\mathrm{cm}^{-2} \mathrm{~s}^{-1}$ at the bare terminus positions. Transit times of less than $2 \mathrm{~s}$ are achieved between the reactor and the counting station located outside the reactor containment area at a distance of about $80 \mathrm{ft}$. Helfer and others (1982) described the P-tube system design. The four tubes are driven simultaneously from a single blower manifold, which is equipped with signal-actuated air-flow valves to control the transit direction in each tube. Through-beam photocell sensors, located at the sending station and the reactor tank boundary, sense the passing of irradiation capsules in both directions. A computercontrollable sample-changer device located just above each counting assembly serves as an automatic sampleloading device and directs returning irradiated samples into the counter and pneumatically ejected samples from the counter to a discard facility or to the load magazine of another P-tube for a second irradiation cycle.

The neutron-counter assemblies have been described in detail by Millard and Keaten (1982). Each assembly consists of a cylindrical arrangement of six $\mathrm{BF}_{3}$ gas-filled neutron-detector tubes surrounding a lead- shielded sample position. The detector tubes are embedded in paraffin, which serves to moderate neutrons emitted from a sample to an optimum energy for detection. The gamma-ray-counting response of this arrangement is very low compared to the neutron response because the $\mathrm{BF}_{3}$ detectors are relatively insensitive to gamma-rays and the lead shield $(6 \mathrm{~cm}$ thick) stops a large fraction of the gamma-rays emitted from a sample without significant neutron absorption. Each counter assembly exhibits a neutron-counting efficiency of about 15 percent and a counter recovery deadtime of $9 \mu$ s following each response.

A CTD unit is located at the reactor operator's console. The CTD unit is a 16K RAM single-board microprocessor that provides individually memorymapped input-output (I/O) capabilities for inputting system condition signals and outputting system control or status signals. Upon request from the counting system minicomputer, the CTD unit controls all aspects of the P-tube operation cycle according to programmed instructions and preset parameters, such as irradiation time and number of capsules loaded. In addition, it monitors all photocell sensor signals from each P-tube to determine irradiation start and end times and transit position. Video display units, located at the reactor console and at the counting station, display the number of samples in each system and the status of each current P-tube cycle. If an 
improper sequence of system condition input signals occurs, then a diagnostic error message is displayed.

A counting system minicomputer sequences the facility operations. The computer is a DEC PDP $8 / F$ with a specially designed $\mathrm{I} / \mathrm{O}$ interface. This interface unit is built up from various signal to logic gates and line drivers that interface with the computer data bus to allow internal I/O of logic bits that can be read and tested by a FORTRAN program. The interface also contains synchronous up-down counters for receiving neutroncounter data similar to a multichannel scaler. The system computer actuates the sample changer operation, signals the CTD unit to initiate an irradiation cycle, and collects and records all pertinent data into appropriate sample information files. The data, on disk, are reduced off line by the same system minicomputer.

\section{ANALYTICAL PROCEDURES}

The general DN approach employs equivalent, sequential irradiation-counting cycles for each sample within an experiment set, which consists of uranium, thorium, and oxygen standards and sample aliquots. The analysis is performed directly on whole sample aliquots without any preirradiation or postirradiation chemical processing. Even though the DN method is relatively free from direct matrix interferences, the specific procedure(s) used to achieve selective measurement of $U$ and (or) Th in a particular experiment may depend on numerous factors related to the experiment-the elemental composition and type of matrix, the amount of sample available, and the desired data confidence. However, for the routine analysis of common or moderately mineralized silicate materials, U-Th ores, and highly carbonaceous (coal-type) samples, the following analysis procedure is widely applicable and most appropriate.

\section{Experiment Organization}

Samples for DN analysis are organized into experiment sets consisting of up to 20 samples along with uranium, thorium, and oxygen standards of appropriate elemental content and matrix type. The DN system programming is structured to measure sequentially each sample and standard within a set under identical experimental conditions. However, to assure that the measurement parameters are intrinsically equivalent for all samples and are within the operational limitations of the system, it is necessary to restrict the samples within an experimental set to a single matrix or compositional category, which is represented by the standards. With respect to DN analysis, most geologic materials fall into one of the following categories:
1. Silicates that contain from 0.1 to $300 \mathrm{ppm} U$ and from 1 to $3,000 \mathrm{ppm}$ Th are irradiated at maximum neutron flux and maximum sample size,

2. High $U$ and (or) Th ores that contain in excess of 0.03 percent $U$ or 0.3 percent $T h$ require appropriate reduction in irradiation fluence and correspondingly higher level standards,

3. Highly carbonaceous materials, such as coal, are run with carbon matrix standards to equivalence potential neutron thermalization within the sample matrix, and

4. Samples that are extremely anomalous in $\mathrm{Be}, \mathrm{F}, \mathrm{Li}, \mathrm{B}$, $\mathrm{Cd}$, and (or) $\mathrm{Gd}$ require special analytical treatment to correct for potential nonfission neutron interferences $(\mathrm{Be}, \mathrm{F})$ or neutron shielding within the sample.

Preanalysis screening of samples into appropriate experimental sets is based on a general knowledge of the geologic setting or rock-type attributes, on equivalent uranium radioactivity estimates, or, if necessary, on a preliminary, semiquantitative assay.

\section{Sample Preparation}

Two-dram polyethylene snap-top vials are filled with powdered or granulated sample material. The vial capacity $\left(7.5 \mathrm{~cm}^{3}\right)$ is approximately $10 \mathrm{~g}$ of powdered silicate material. The filled vials are weighed on an analytical balance that has been tared for the weight of an empty vial. The uncertainty of this tare is about $50 \mathrm{mg}$, or 0.5 percent for a $10-\mathrm{g}$ sample. For samples significantly less than $10 \mathrm{~g}$, the individual vials are tared, filled, and weighed. The vials are heat sealed and placed into screwtop polyethylene capsules (rabbits), which are compatible with the pneumatic transfer system.

A set of reusable uranium standards have been prepared by homogeneously doping a low-uranium rock powder (dunite DTS-1, which contains 3 ppb U and 10 $\mathrm{ppb} \mathrm{Th}$ ) with uranium standard solutions prepared from isotopically normal uranium oxide (National Bureau of Standards SRM 950a). Thorium-doped standards were prepared in a similar fashion by using standard solutions prepared from reagent-grade thorium nitrate. Aliquots of these doped materials, sealed in 2-dram polyvials, constitute a set of reusable working standards that are matrix similar with respect to DN analysis of silicates. Similar working standards also have been prepared on a coal matrix. These working standards were calibrated further against a set of standard reference rocks for which reliable literature values (such as isotope dilution-mass spectrometry data) are available.

\section{Irradiation Counting}

A set of sample irradiation capsules are loaded into an automatic sample changer magazine in the order of a 
serially coded sample information file (run file) residing on the system computer disk. An automatic run is initiated by appointing the system parameters-run-file identification, designation of standards, serial number of first sample, and number of samples. During the run, each sample is sequenced through two irradiation-counting cycles. For the first cycle, a sample is pneumatically transferred to a cadmium lined (fast flux) irradiation terminus, irradiated for $1 \mathrm{~min}$, and returned to the neutron-counting assembly. After a decay period of $5 \mathrm{~s}$ following the end of irradiation, the sample is counted for a 5-s interval (short decay count) and then counted again for a 60 -s interval beginning $20 \mathrm{~s}$ after irradiation (long decay count). Counting data for each count are recorded independently on a disk along with the actual irradiation and transit times. The rabbit advances to the load position of another P-tube system for a second irradiation- counting cycle utilizing a bare (thermal flux) irradiation terminus. The irradiation, decay, and counting schedule is identical to that of the first irradiation. The analytical parameters for a complete cycle of two irradiations and two countings are shown in table 2.

\section{Data Reduction}

Subsequent to the completion of one, or several, sample set(s), the data residing on the system computer disk are reduced on the same computer using an autosequencing data-reduction program. Each count is reduced to a counts per second value for the counting interval, corrected for counter dead time and background, and normalized to an irradiation time of exactly $60.0 \mathrm{~s}$. From the counting data for each standard, the interference-free

Table 2. Analytical parameters for a complete cycle of two irradiations and appropriate counting sequence using the U.S. Geological Survey delayed neutron analysis facility with the reactor operating at 1-megawatt power

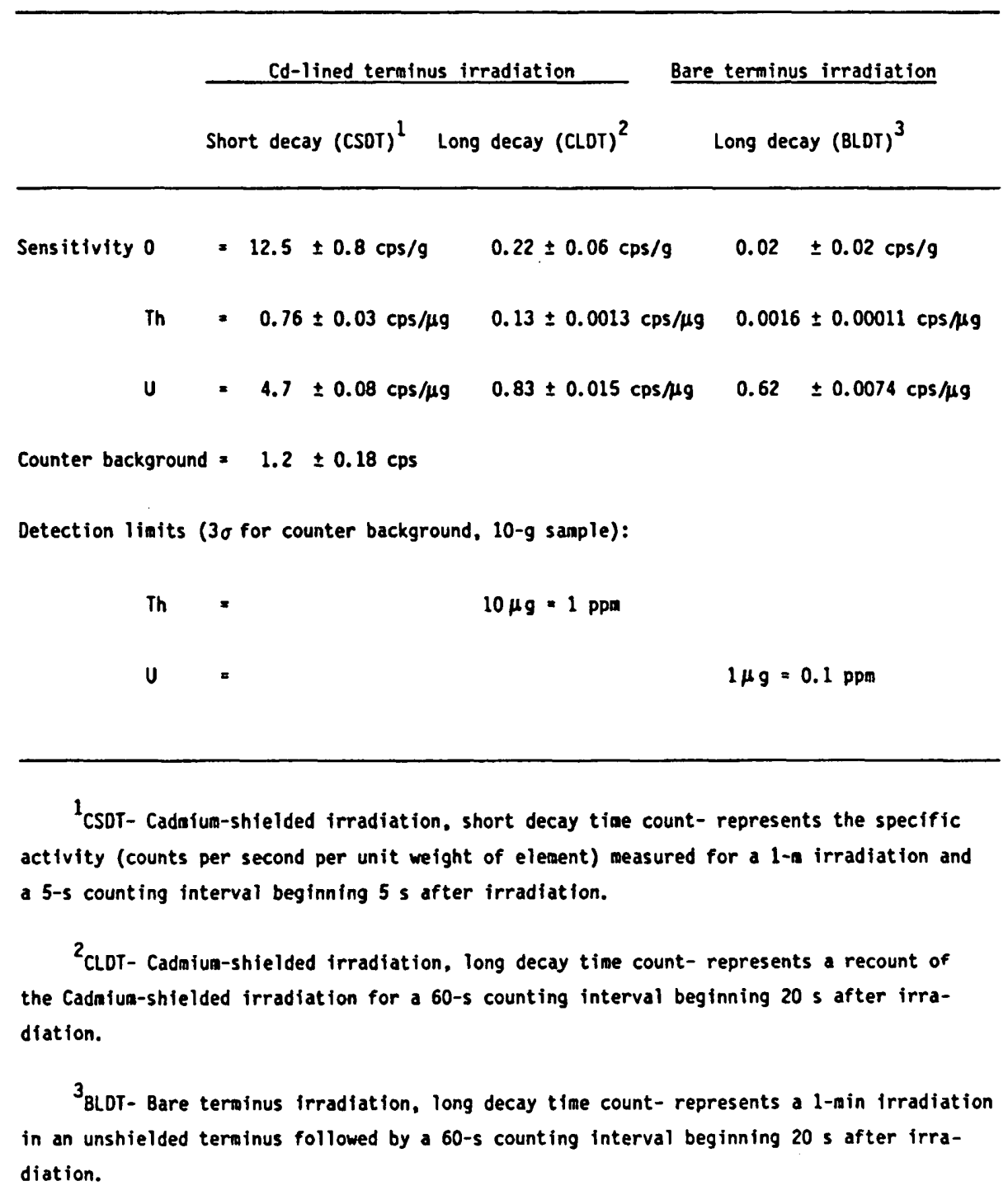


specific activity (counts per second per unit weight of the element) for the element of that standard is computed for each of the counting intervals. An iterative algorithm then is applied to the counting data for each sample to resolve interelement interferences, assuming, as a first approximation, that the bare terminus-long decay counts are due primarily to uranium and that the cadmium-shielded long decay counts, after correction for the apparent uranium interference, represent the thorium contribution. After resolution of the individual element contributions, the concentrations of oxygen, uranium, and thorium in the sample are computed. Oxygen, however, is not reported because of the large uncertainty in the computed value. An estimate of analytical precision is computed for each measurement based on uncertainties due to sample weighing, standard values, counting statistics for the sample and background, and the magnitude of interelement interference corrections. The precision is reported as the coefficient of variation, which represents one standard deviation expressed as a percentage of the concentration value.

\section{DISCUSSION}

\section{Detection Limits}

As shown in table 2, the experimentally measured delayed neutron count rate for $U$ is considerably greater than that measured for an equal quantity of Th under all irradiation-counting conditions. This difference is due largely to differences in their fission cross sections. Consequently, the DN method is inherently more sensitive to the measurement of uranium than to the measurement of thorium. Comparing the 3- $\sigma$ uncertainty of the counter background with the U sensitivity (BLDT count, table 2) yields an absolute detection limit of $1 \mu \mathrm{g} \mathrm{U}$, which corresponds to $0.1 \mathrm{ppm} \mathrm{U}$ in a $10 \mathrm{~g}$ sample. For $\mathrm{Th}$, the minimum detectable count rate depends on counter background plus uncertainties in correcting the gross counts for oxygen and uranium contributions (CLDT count, table 2). For samples having a $T h$ to $U$ ratio greater than 3 , the 3- $\sigma$ detection limit for Th is about $10 \mu \mathrm{g} \mathrm{Th}$, or 1 $\mathrm{ppm}$ for a 10-g sample. The detection limit for Th is correspondingly higher if the $\mathrm{Th}$ to $\mathrm{U}$ ratio is less than 3 . Thorium is not measured reliably, even at high levels, if the $T h$ to $U$ ratio is less than 1 .

\section{Precision and Accuracy}

Millard and Keaten (1982) have evaluated thoroughly the precision of the DN procedure as a function of the concentrations of uranium and thorium. They examined the data for duplicate analyses of three different sets of samples (a total of 588 samples) ranging from about
0.2 to $8,000 \mathrm{ppm} \mathrm{U}$ and from 1 to $400 \mathrm{ppm}$ Th. The data for each set of duplicates were grouped by uranium or thorium concentration decades. The data within each decade were then treated by the Geological Survey Statpac Program (Van Trump and Miesch, 1977), which uses Youden's equation (Youden, 1951) to compute the standard deviation of a single determination from sets of duplicates. The average relative standard deviation (coefficient of variation) for each concentration decade plotted against the mean concentration for that interval is illustrated by points in figure 2 . The curves shown in figure 2 represent an estimate of the coefficient of variation computed from the counting statistics and are not to be interpreted as fits to the experimental data for the duplicates (points). However, the experimental data match these curves quite well, which suggests that the precision of DN measurements is controlled primarily by counting statistics.

As indicated by these data, an analytical precision of \pm 5 percent, or better, generally is observed for the measurement of uranium concentrations greater than about $1 \mathrm{ppm}$ in a $10 \mathrm{~g}$ sample aliquot. Quantitative, but less precise, data usually are derived from a single measurement of lesser concentrations down to a realistic determination limit of about $0.2 \mathrm{ppm} U$, which is represented by an expected uncertainty of \pm 30 percent. The precision of thorium measurements is strongly dependent on the $\mathrm{Th}$ to $\mathrm{U}$ ratio and, therefore, not as predictable before analysis. For the analysis of materials having a Th to $U$ ratio greater than 3 , a single measurement precision of about \pm 10 percent, or better, generally is expected for thorium concentrations on the order of $10 \mathrm{ppm}$, and a determination limit $[ \pm 30$ percent coefficient of variation (CV)] of about 2-3 ppm Th generally is achieved.

\section{Accuracy}

Standard reference rocks are not run routinely with each DN analysis set to evaluate the analytical accuracy. Periodically, a set of reference rocks is run against the working standards to evaluate the accuracy of the standard calibrations. The accuracy of DN data also is tested occasionally in collaboration with various submitters who also have obtained reliable data by other analytical techniques.

In table 3, uranium and thorium values obtained from the analysis of a set of Geological Survey standard rocks are compared with values compiled from the literature. The DN values are taken from Millard (1976) and result from the analysis of six aliquots of each sample. These data illustrate the excellent level of accuracy and precision that can be achieved for uranium by replicate analyses. The disparity among granodiorite GSP-1 values may be due to sample inhomogeneity, which has a great 


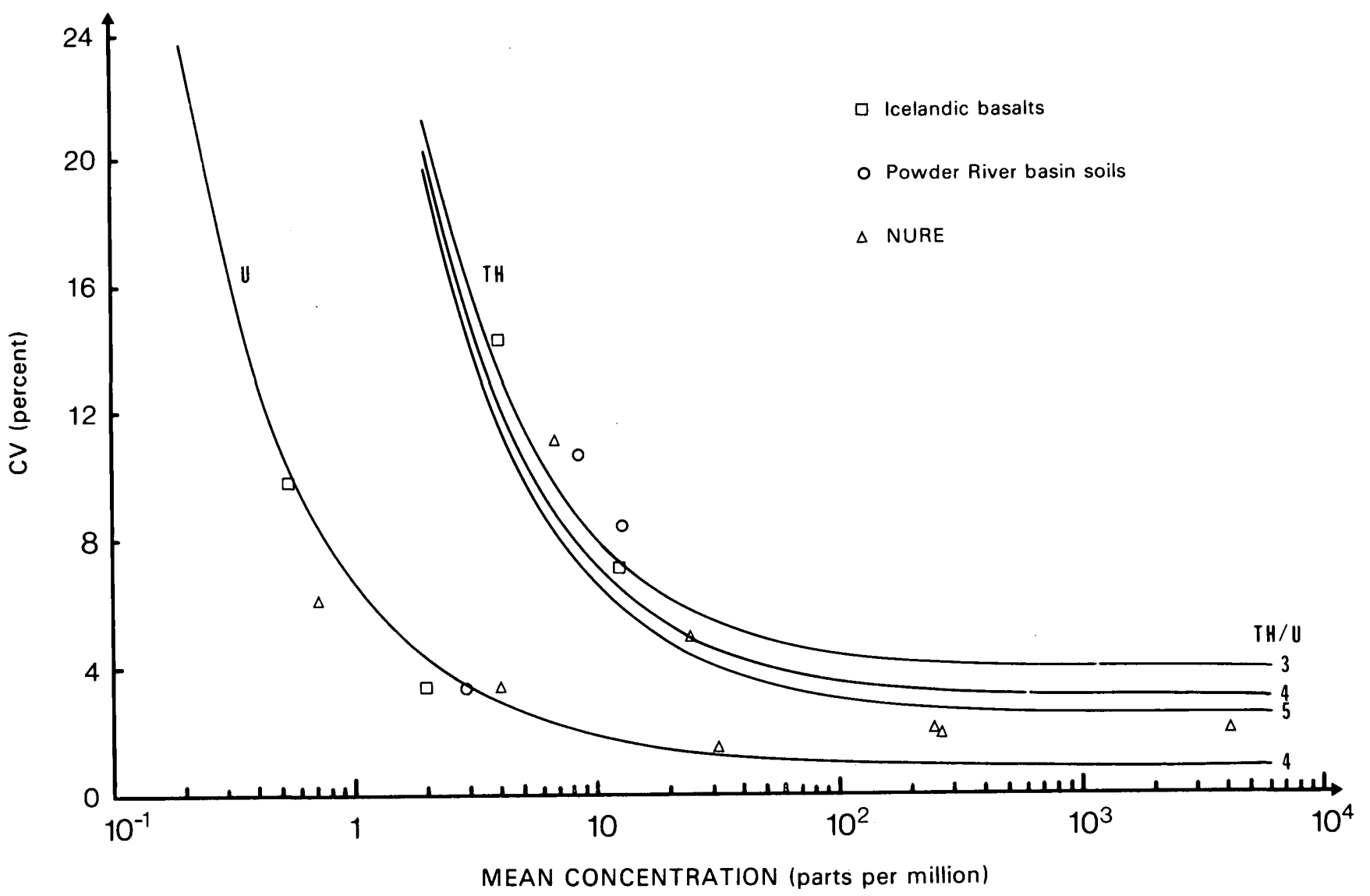

Figure 2. Average coefficient of variation for each decade interval of concentration plotted against the average concentration for the decade. Curves represent coefficient of variation computed from counting statistics; points are experimental data. NURE, National Uranium Resource Evaluation Program. (Millard and Keaten, 1982.)

effect on analytical methods using a small sample, but little effect on DN analysis using $10 \mathrm{~g}$ of sample. Thorium data in table 3 illustrate the variability of both accuracy and precision depending on the level of thorium and the Th to $U$ ratio. The accuracy of the data generally is represented by the precision of the measurement when the Th concentration and the $T h$ to $U$ ratio are relatively low. In this case, small uncertainties in uranium due to poor counting statistics lead to a large error in the thorium value.

Perhaps a more useful evaluation of data quality is derived from single-analysis results because the routine application of the DN method does not utilize replicate analyses. In table 4 , single-analysis DN data are compared with reference values for a set of reference samples that are analyzed periodically during a normal run. The DN data and computed CV are taken directly from a typical run (latest reference set run) without censorship. Except for limitations discussed herein, the ratios of DN to reference values indicate no significant bias for either $\mathrm{U}$ or $\mathrm{Th}$, and lesser reference agreement is usually within the 2- $\sigma$ uncertainty of the measurement as computed from counting statistics. These results, typical of numerous such comparisons (for example, Stuckless and others, 1977), support a conclusion that single-analysis uranium data generally are precise and accurate within \pm 5 percent and that thorium data are precise and accurate within \pm 10 percent for samples exhibiting appropriate concentration levels and $T h$ to $U$ ratios.

\section{Interferences and Limitations}

The only direct source of nonfission delayed neutron interferences is the activation of ${ }^{17} \mathrm{O}$ and ${ }^{9} \mathrm{Be}$, as described above. These interferences are insignificant in the analysis of geologic materials because oxygen is accounted for by standardization and $\mathrm{Be}$ is low in abundance and its activation product is very short lived. A neutron-counting interference, indistinguishable from DN signals, may arise from extreme levels of very high energy gamma-rays emitted from a sample interacting with their surroundings by a $(\gamma, \mathrm{n})$ reaction. The $(\gamma, \mathrm{n})$ cross sections for major and minor elements in a rock matrix are so small that only highly anomalous Be content is considered a potential problem. However, gamma interactions with 
Table 3. Uranium and thorium values obtained by replicate delayed neutron analyses compared to published consenses values

[RSD: percent relative standard deviation; DN: delayed neutron counting]

\begin{tabular}{|c|c|c|c|c|c|c|c|c|c|c|c|}
\hline \multirow[b]{2}{*}{ Sample } & \multirow[b]{2}{*}{ Rock type } & \multicolumn{4}{|c|}{ Uraniun } & \multicolumn{6}{|c|}{ Thoriun } \\
\hline & & $\begin{array}{l}\text { Literature } \\
\text { value (ppra) }\end{array}$ & $\begin{array}{l}\text { DH value } \\
\text { (ppm) }\end{array}$ & $\begin{array}{c}\text { ON } \\
\text { (RSD) } \\
\text { (percent) }\end{array}$ & $\frac{D M}{\begin{array}{l}\text { literature } \\
\text { ratio }\end{array}}$ & $\begin{array}{l}\text { Litera } \\
\text { value }\end{array}$ & $\begin{array}{l}\text { ature } \\
\text { (ppn) }\end{array}$ & $\begin{array}{l}\text { ON V } \\
\text { (ppr }\end{array}$ & $\begin{array}{l}\text { alue } \\
\text { m) }\end{array}$ & $\begin{array}{c}\text { DN } \\
\text { (RSD) } \\
\text { (percent) }\end{array}$ & $\begin{array}{c}\text { DN } \\
\text { Iiterature } \\
\text { ratio }\end{array}$ \\
\hline$G-2$ & Granite & $2.04 \pm 0.17$ & $2.15 \pm 0.07$ & 3.2 & 1.05 & 24.6 & \pm 1.5 & 24.0 & \pm 0.9 & 3.6 & 0.98 \\
\hline GSP-1 & Granodiorite & $2.20 \pm 0.3$ & $2.56 \pm 0.10$ & 3.7 & 1.16 & 105 & \pm 5 & 107 & \pm 2.2 & 2.0 & 1.02 \\
\hline$A G V-1$ & Andesite & $1.89 \pm 0.25$ & $2.05 \pm 0.09$ & 4.4 & 1.08 & 6.5 & \pm 0.4 & 5.37 & \pm 0.9 & 16 & 0.83 \\
\hline$B C R-1$ & Basalt & $1.71 \pm 0.16$ & $1.81 \pm 0.06$ & 3.3 & 1.05 & 6.04 & \pm 0.6 & 5.26 & \pm 0.8 & 16 & 0.87 \\
\hline$S T M-1$ & Syenite & $9.07 \pm 0.07$ & $9.10 \pm 0.14$ & 1.5 & 1.00 & 33 & \pm 5 & 26.6 & \pm 1.2 & 4.5 & 0.81 \\
\hline$R G M-1$ & Rhyolite & $5.84 \pm 0.07$ & $5.85 \pm 0.10$ & 1.7 & 1.00 & 16 & \pm 2 & 13.1 & \pm 1.5 & 11 & 0.82 \\
\hline QLO-1 & Quartz latite & $1.97 \pm 0.03$ & $2.01 \pm 0.10$ & 5.2 & 1.02 & 4.6 & \pm 1.1 & 3.24 & \pm 0.8 & 24 & 0.70 \\
\hline SCo-1 & Shale & $3.09 \pm 0.14$ & $3.15 \pm 0.09$ & 3.0 & 1.02 & 10.1 & \pm 0.7 & 9.52 & \pm 0.6 & 6.2 & 0.94 \\
\hline MAG-1 & Marine nud & $2.85 \pm 0.02$ & $2.82 \pm 0.10$ & 3.5 & 0.99 & 12.8 & \pm 0.5 & 12.2 & \pm 0.8 & 6.4 & 0.95 \\
\hline SO-1 & Mica schist & $3.02 \pm 0.10$ & $3.12 \pm 0.08$ & 2.6 & 1.03 & 12.0 & \pm 0.4 & 11.4 & \pm 0.8 & 7.1 & 0.95 \\
\hline BHVO-1 & Basalt. & $0.42 \pm 0.06$ & $0.48 \pm 0.07$ & 15 & 1.13 & 1.1 & \pm 0.2 & 0.9 & \pm 0.7 & 72 & 0.8 \\
\hline SGR-1 & Shale & $5.57 \pm 0.15$ & $5.60 \pm 0.22$ & 4.0 & 1.01 & 4.9 & \pm 0.1 & 7.66 & \pm 1.9 & 25 & 1.56 \\
\hline
\end{tabular}

\footnotetext{
$1_{\text {Literature values for the first four rocks are fron Gladney and Burns (1983) and values for renaining rocks }}$ are fron Gladney and Goode (1981).
}

deuterium in the paraffin detector housing is a potential interference in the analysis of samples high in fluorine. Gamma-rays resulting from the activation of fluorine in a fluorite sample (50 percent $\mathrm{F}$ ) produces an interference equivalent to $2.8 \mathrm{ppm} \mathrm{U}$ and $10 \mathrm{ppm}$ Th. Fluorine interferences could be corrected, but the potential radiation hazard associated with the high gamma activity makes it impractical to run samples containing more than 10 percent fluorine. The effect of lower energy gammarays normally prevalent in an activated sample, such as those of ${ }^{28} \mathrm{Al}$ and ${ }^{56} \mathrm{Mn}$, is negligible with our leadshielded counting assembly.

An anomalously high concentration of elements that exhibit very high neutron-capture cross sections, such as $\mathrm{Li}, \mathrm{B}, \mathrm{Cd}$, and $\mathrm{Gd}$, can affect seriously the accuracy of DN results because of neutron shielding (flux depression) within the sample. The normal DN procedure does not account for these effects; therefore, samples of this type are not appropriate for DN analysis without special analytical treatment. Similarly, the analysis of highly carbonaceous materials, such as coal, may yield erroneously high Th values due to fast neutron thermalization within the sample. The apparent excess Th due to $6 \mathrm{~g}$ of a coal matrix is equivalent to about 0.5 times the $U$ concentration. To minimize this error, samples must be designated as carbonaceous and run with standards of equivalent matrix. 
Table 4. Comparison of single-analysis delayed neutron data with reference values from a set of reference samples analyzed during a normal run

[Coefficient of variation (CV) computed for each analysis based on 1- $\sigma$ uncertainty in counting statistics, expressed as percentage of concentration; DN: delayed neutron counting]

\begin{tabular}{|c|c|c|c|c|c|c|c|c|c|}
\hline \multirow{2}{*}{$\begin{array}{l}\text { Sample } \\
\text { designation }\end{array}$} & \multirow{2}{*}{$\begin{array}{c}\text { Reference } \\
\text { note }\end{array}$} & \multicolumn{4}{|c|}{ Uranium } & \multicolumn{4}{|c|}{ Thorium } \\
\hline & & ON & CV & Reference & Ratio & DN & cv & Reference & Ratio \\
\hline$k-7$ & 1 & 0.27 & 9 & 0.27 & 1.00 & $<0.9$ & -- & 0.57 & \\
\hline$W-1$ & 2 & 0.55 & 6 & 0.57 & .96 & 2.90 & 14 & 2.40 & 1.20 \\
\hline Hinsdale basalt & $t 1$ & 0.87 & 4 & 0.88 & 99 & 3.63 & 12 & 3.50 & 1.04 \\
\hline BCR-1 & 2 & 1.72 & 3 & 1.71 & 1.01 & 6.62 & 9 & 6.04 & 1.10 \\
\hline GSP-1 & 2 & 2.52 & 3 & 2.2 & 1.15 & 113 & 2 & 105 & 1.07 \\
\hline$F F-4$ & 1 & 3.27 & 2 & 3.07 & 1.07 & 9.24 & 8 & 10.4 & 0.89 \\
\hline JNR-6379 & 1 & 7.85 & 2 & 7.59 & 1.03 & $<3$ & -- & 3.01 & -- \\
\hline RN-1 & 1 & 15.9 & 1 & 15.3 & 1.04 & 39.7 & 4 & 37.7 & 1.05 \\
\hline 3633 & 1 & 24.2 & 1 & 23.4 & 1.03 & 87.5 & 3 & 82.0 & 1.07 \\
\hline $60-5-4-1$ & 1 & 30.2 & 1 & 30.6 & 0.99 & $<7$ & -- & 22.0 & -- \\
\hline AEC-NBL-80 & 3 & 40.7 & 1 & 40 & 1.02 & 1,060 & 1 & 1.000 & 1.06 \\
\hline$A E C-N B L-76 A$ & 3 & 105 & 1 & 101 & 1.04 & -- & -- & -- & -- \\
\hline$A E C-N B L-1$ & 3 & 244 & 1 & 250 & 0.98 & -- & -- & -- & -- \\
\hline AEC-NBL-74 & 3 & 960 & 1 & 1,000 & 0.96 & -- & -- & -- & -- \\
\hline Average ratio & & & & & $1.02 \pm$ & 0.05 & & & 1.06 \\
\hline
\end{tabular}

Reference notes:

1-Isotope dilution-mass spectrometry literature values as cited by Millard (1976).

2-Compilation of literature values for standard rocks by Gladney and Burns (1983).

3-Synthetic standard preparation value. 


\section{SUMMARY}

The delayed neutron counting technique is an extremely useful tool for measuring $U$ and $\mathrm{Th}$ in a wide variety of geological materials. It yields precise and accurate results for trace-level concentrations yet exhibits a high degree of practical expediency. These features make it desirable for large-scale applications, such as geochemical exploration and resource reconnaissance. Thorium results are not as precise or accurate as the uranium determinations but are usually adequate for these types of applications. If more detailed interpretations are desired, then an alternate technique for $\mathrm{Th}$ is advised. Analytical features of the DN procedure as routinely applied to the analysis of geologic materials are summarized in table 5 .

Table 5. Summary of analytical features of delayed neutron analysis of geologic materials

Elements measured: $U$ and Th.

Suitable matrices: Most silicate rocks, soils, and sone noderately mineralized (low uranium) ores. Uranium ores and carbonaceous materiais if designated as such.

Matrix IImitations: Samples highly anonalous in F, Be, L1, B, Cd, or Gd.

Other limitations: Decreased sensitivity and confidence for $U$ if the Th to $U$ ratio is greater than 50: similarity, for th if the th to $U$ ratio is less than 3.

Sensitivity:

Detection limits are $1 \mu \mathrm{g}$ and $10 \mu \mathrm{g} \mathrm{Th}$. which corresponds to $0.1 \mathrm{ppm} U$ and $1 \mathrm{ppn}$ Th using a $10-g$ sample.

Sample requirements: Aliquot volume of about $7.5 \mathrm{cc}$ of powdered or granulated material which corresponds to about $10 \mathrm{~g}$ of silicate and $6 \mathrm{~g}$ of coal. Smaller samples may be analyzed with decreased sensitivity.

Quality control: Periodic analys is of a set of references rocks (see table 4 ).

Throughput:

Realistic throughput of 400 samples per week per full time analyst. Instrunent capacity about 400 samples per operation day.

\section{REFERENCES CITED}

Amiel, Saadia, 1962, Analytical applications of delayed neutron emission in fissionable elements: Analytical Chemistry, v. 34 , p. $1683-1692$.

Brownlee, J. L., Jr., 1968, The detection and determination of fissionable species by neutron activation-delayed neutron counting, in DeVoe, J. R., ed., Modern trends in activation analysis: NBS Special Publication 312, v. I, p. 495-500.

Dyer, F. F., Emery, J. F., and Leddicotte, G. W., 1962, A comprehensive study of the neutron activation analysis of uranium by delayed neutron counting: Report ORNL-3342, U.S. Atomic Energy Commission, 69 p.

Echo, M. W., and Turk, E. H., 1957, Quantitative determination of U-234 by delayed neutron counting: Report PTR143, U.S. Atomic Energy Commission, 21 p.

Gale, N. H., 1967, Development of delayed neutron technique as a rapid and precise method for determination of uranium and thorium at trace levels in rocks and minerals with applications to isotope geology: Symposium on Radioactive Dating and Methods of Low Level Counting, Vienna, Austria, 1967, Proceedings, p. 431-52.

Gladney, E. S., and Burns, C. E., 1983, 1982 Compilation of elemental concentrations in eleven United States Geological Survey Rock Standards: Geostandards Newsletter, v. 7, p. 3-226.

Gladney, E. S., Curtis, D. B., Perrin, D. R., Owens, J. W., and Goode, W. E., 1980, Nuclear techniques for chemical analysis of environmental materials: Los Alomos Scientific Laboratory Report, LA-8192-MS, p. 30-52.

Gladney, E. S., and Goode, W. E., 1981, Elemental concentrtions in eight new U.S.G.S. rocks-A review: Geostandards Newsletter, v. 5, p. 31-64.

Helfer, P. G., Millard, H. T., Jr., and Zermane, A. J., 1982, Automated pneumatic transfer irradiation system for delayed neutron counting: The Eighth Biennial U.S. TRIGA Users Conference, Idaho Falls, Idaho, 1982, Proceedings, section 3, p. 17-22.

Laul, J. C., 1979, Neutron activation analysis of geological materials: Atomic Energy Review, v. 17, p. 603-695.

MacMurdo, K. W., and Bowman, W. W., 1977, Assay of fissile materials by a cyclic method of neutron activation and delayed neutron counting: Nuclear Instruments and Methods, v. 141, p. 299-306.

Millard, H. T., Jr., 1976, Determination of uranium and thorium in U.S.G.S. standard rocks by the delayed neutron technique, in Flanagan, F. J., ed., Descriptions and analysis of eight new USGS rock standards: U.S. Geological Survey Professional Paper 840, p. 61-70.

Millard, H. T., Jr., and Keaten, B. A., 1982, Precision of uranium and thorium determinations by delayed neutron counting: Journal of Radioanalytical Chemistry, v. 72, p. 489-500.

Rosenberg, R. J., Pitkanen, V., and Sorsa, A., 1977, An automatic uranium analyzer based on delayed neutron counting: Journal of Radioanalytical Chemistry, v. 37, p. 169-179. 
Stuckless, J. S., Millard, H. T., Jr., Bunker, C. M., Nkomo, I. T., Rosholt, J. N., Bush, C. A., Huffman, C., Jr., and Keil, R. L., 1977, A comparison of some analytical techniques for determining uranium, thorium, and potassium in granitic rocks: U.S. Geological Survey Journal of Research, v. 51, no. 1 , p. 83-91.
Van Trump, George, Jr., and Miesch, A. T., 1977, The U.S. Geological Survey RASS-STATPAC system for management and statistical reduction of geochemical data: Computers and Geoscience, v. 3, p. 475-488.

Youden, W. J., 1951, Statistical methods for chemists: New York, John Wiley, p. 16. 
CHAPTER J

Radiochemical Neutron Activation Analysis of Geologic Materials

\author{
By G. A. WANDLESS
}

U.S. GEOLOGICAL SURVEY BULLETIN 1770

Methods for Geochemical Analysis 



\title{
CONTENTS
}

\author{
Abstract J1 \\ Introduction J1 \\ Experimental J1 \\ Sample preparation $\mathbf{J 1}$ \\ Carriers J1 \\ Sample decomposition J1 \\ Radiochemical separations J2 \\ Rare-earth element group separation $\mathbf{J 3}$ \\ Siderophile and volatile elements $\mathbf{J 3}$ \\ Radioassay $\mathbf{J 3}$ \\ Yield determinations $\mathbf{J 5}$ \\ Detection limits, precision, and accuracy $\mathbf{J 5}$ \\ Summary J8 \\ References cited J8
}

\section{FIGURES}

1. Comparison of steps in radiochemical neutron activation analysis and instrumental neutron activation analysis $\mathbf{J 2}$

2. Elements commonly determined by radiochemical neutron activation analysis in geological samples $\mathbf{J 2}$

3. Group separation of the rare-earth elements used at the U.S. Geological Survey's National Center J3

4. Gamma-ray spectrum of basalt BCR-1 after radiochemical neutron activation analysis separation of the rare-earth elements. J4

5. Radiochemical neutron activation analysis of siderophile and volatile elements J6

\section{TABLE}

1. Detection limits for various elements by radiochemical neutron activation analysis $\mathbf{J 8}$ 



\title{
Radiochemical Neutron Activation Analysis of Geologic Materials
}

\author{
By G. A. Wandless
}

\section{Abstract}

The technique of radiochemical neutron activation analysis provides ultimate sensitivity for neutron activation analysis by removing the matrix interferences that result in higher detection limits for the instrumental method. Removal of major activities following neutron activation also extends the technique to more elements than are detectable by instrumental neutron activation analysis. The precision and accuracy of radiochemical neutron activation analysis for nearly all elements considered here is $1-10$ percent. Sensitivity is dependent on the nuclear properties of the elements of interest and their activation products. This method is labor intensive, requiring chemists to perform chemical separations before radiation measurements. The method is also destructive in that the sample is consumed.

\section{INTRODUCTION}

Radiochemical neutron activation analysis (RNAA) is a technique used when a desired element or elements cannot be detected or determined accurately by instrumental neutron activation analysis (INAA) or other instrumental techniques due to interferences from matrix elements. The elements of interest are isolated from the major activation products (for example, ${ }^{56} \mathrm{Mn},{ }^{46} \mathrm{Sc},{ }^{59} \mathrm{Fe}$, ${ }^{60} \mathrm{Co}$ ) found in neutron irradiated geologic samples. This radiochemical processing may isolate the indicator radionuclide(s) of a single element or group of elements. The gamma-ray spectra of these isolated radionuclides are free of gamma-ray lines of matrix components, thus reducing the background level, improving accuracy, and lowering detection limits. Some elements have no suitable isotopes with gamma-ray lines (for example, $\mathrm{Tl}, \mathrm{Bi}$ ), and the activity of their activation products is measured by beta counting.

As shown in figure 1, the five basic steps for RNAA are sample preparation, neutron activation, radiochemical separations, radioactivity measurements, and yield determinations. Each step will be described in more detail below. Figure 2 shows those elements commonly determined by RNAA.

\section{EXPERIMENTAL}

\section{Sample Preparation}

The usual precautions must be taken to avoid contamination of the sample before irradiation. Samples can be weighed into aluminum foil packets, polyethylene vials, or silica tubes. The latter two are heat sealed to prevent contamination during irradiation.

\section{Carriers}

The amount of a trace element found in geologic samples is typically in the microgram to picogram range, too small to be carried through standard chemical processing techniques. Therefore, a known amount $(10 \mathrm{mg})$ of each element of interest is added before chemical separations begin, allowing separations of milligram amounts of these elements. Because the chemical yield can be measured after processing by simple analytical techniques (gravimetric, atomic absorption, X-ray fluorescence, reactivation, active tracer), the chemical separation need not be quantitative. However, because the chemical yield factor is used to correct the counting data, it is essential that this carrier be in isotopic equilibrium with the irradiated elements. Normally, this is achieved because of the high mobility of ions at fusion temperatures. The carrier technique also can be used to remove trace amounts of interfering isotopes. Addition of "holdback carriers" allows effective separation of those elements. Aliquots of each carrier solution are measured into the fusion crucible and dried before the addition of the sample.

\section{Sample Decomposition}

Geologic samples can be decomposed by fusion with $\mathrm{Na}_{2} \mathrm{O}_{2}$ and $\mathrm{NaOH}$ or by dissolution with acids. 


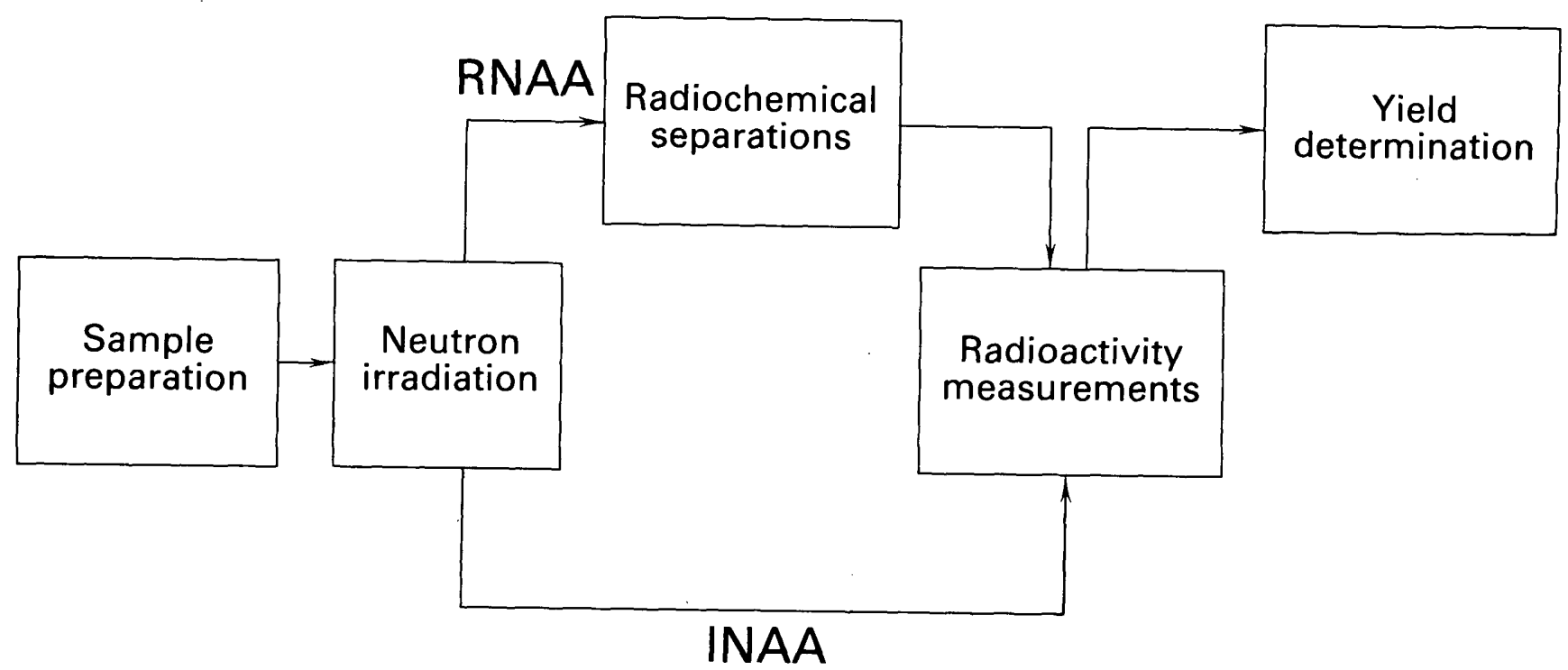

Figure 1. Comparison of steps in radiochemical neutron activation analysis (RNAA) and instrumental neutron activation analysis (INAA) (from Chou, 1980).

Fusion generally involves addition of 5-10 $\mathrm{g}$ of $\mathrm{Na}_{2} \mathrm{O}_{2}$ and $1 \mathrm{~g}$ of $\mathrm{NaOH}$ to the sample. The crucible is covered and heated over a Meker burner or in a furnace for 10-20 min, which insures complete sample decomposition and isotopic equilibrium. Acid decomposition normally is accomplished by the use of $\mathrm{HCl}, \mathrm{HNO}_{3}, \mathrm{HF}$, and $\mathrm{HClO}_{4}$ or combinations of these acids in platinum crucibles or high-pressure bombs.

\section{Radiochemical Separations}

Any analytical separation technique can be applied to radiochemical analysis. Many of the separation techniques developed for spectroscopic analysis and described in Chapter D also can be applied to RNAA. Selection of the proper separation procedure is determined by several factors, including isotope half-lives, interfering elements, the efficiency of the separation, and minimization of radiation exposure to the analyst.

Precipitation is a classical separation technique that may be used to isolate indicator radionuclides from matrix

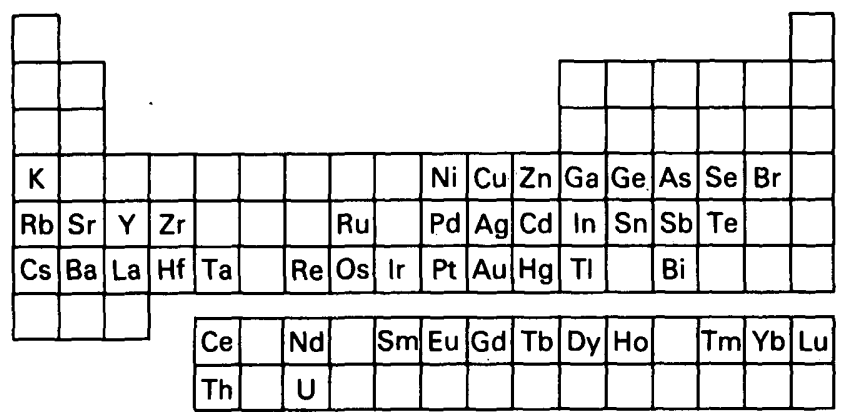

Figure 2. Elements commonly determined by radiochemical neutron activation analysis in geological samples (from Chou, 1980). contaminates; for example, the rare-earth elements (REE) from radioactive sodium by precipitation of their hydroxides. It also can be used to place the desired elements in a form for yield determination and radioassay (for example, precipitation of nickel by dimethylglyoxime), which is collected on tared filter paper, weighed, and mounted for radioassay.

Solvent extraction, a rapid and effective technique, involves shaking an immiscible organic solvent with an aqueous phase containing the radionuclides of interest. Differences in solubility of compounds in the aqueous and organic phases allows separation of a species soluble in an organic phase, such as $\mathrm{GeCl}_{4}$ in $\mathrm{CCl}_{4}$, from interfering species that are not. After mixing, the phases are allowed to separate, and the organic phase containing the nuclide of interest in solution is removed.

Fire assay has a long history as a technique for the extraction of precious metals from ores by fusion with a collector metal. This method has been used for the radiochemical separation of gold by Rowe and Simon (1968), Pd, Pt, Au, Ru, Os, and Ir by Millard and Bartel (1971), and $\mathrm{Cu}, \mathrm{As}, \mathrm{Sb}, \mathrm{Re}, \mathrm{Pt}, \mathrm{Au}, \mathrm{W}$, and Mo by Rammensee and Palme (1982).

Distillation can be used to separate volatile species from nonvolatile species; for example, $\mathrm{RuO}_{4}$ and $\mathrm{OsO}_{4}$ can be distilled from a solution containing nonvolatile compounds.

Ion exchange is a rapid and effective method of radiochemical separation, and the exchange columns can be shielded easily to minimize analyst exposure. The technique utilizes differences in element-exchange properties between a column of synthetic organic resin and a fluid phase. A solution containing multiple elements is "loaded" on the column, the elements of interest are adsorbed onto the ion-exchange resin, and the desired 
elements are eluted selectively with solutions of various $\mathrm{pH}$ or complexing agents. Thus, several desired elements can be adsorbed onto a single column and removed individually. Alternatively, the column also may adsorb the undesired matrix interference elements and allow the desired elements to pass through; for example, in the group separation of the REE described below, Sc is adsorbed on a thiocyanate column, but the REE are not.

\section{Rare-Earth Element Group Separation}

A flow chart of the separation procedure for the REE used at the National Center, U.S. Geological Survey, is shown in figure 3. A detailed procedure is given in Wandless and Morgan (1985) and will be described only briefly here.

Samples of approximately $0.1 \mathrm{~g}$ are weighed into silica vials and sealed. Up to 12 samples, including any control samples, can be irradiated and processed at one time. Samples are irradiated in the central thimble of the U.S. Geological Survey TRIGA reactor in Denver or in the high-flux facility of the University of Missouri Research Reactor. The vials then are opened, the irradiated rock powders placed in crucibles containing dried carriers and digested with $\mathrm{Na}_{2} \mathrm{O}_{2}$ and $\mathrm{NaOH}$. The fusion cake is digested with water, and a series of precipitation and ion-exchange steps are performed to remove matrix contaminants. The purified REE are "loaded" on ionexchange filter papers; gamma-ray spectra then are collected and analyzed by computer as described by Baedecker (1977). Figure 4 shows a gamma-ray spectrum of reference basalt BCR-1 after radiochemical separation of the REE. Ba and Sr also may be determined in this separation scheme. Yields are determined by energydispersive $\mathrm{X}$-ray spectrometry or by reactivation.

\section{Siderophile and Volatile Elements}

The methods used for the determination of the siderophile and volatile elements have been revised extensively from Keays and others (1974). A flow chart of the separation scheme is shown in figure 5. Samples are fused with $\mathrm{Na}_{2} \mathrm{O}_{2}$ and $\mathrm{NaOH}$ in zirconium crucibles containing dried carriers of the elements of interest. The fusion cake is digested with distilled water, and the sulfide precipitated with sodium sulfides. Osmium is distilled from the precipitate fraction, and silver is precipitated from the distillation residue. The supernate is "loaded" onto an ionexchange resin, and $\mathrm{Au}, \mathrm{Bi}, \mathrm{Cd}, \mathrm{Ir}, \mathrm{In}, \mathrm{Tl}, \mathrm{Ni}, \mathrm{Te}$, and $\mathrm{Zn}$ are eluted with various reagents. Germanium is distilled from the fusion cake supernate, and the remaining elements, Se, Te, Sb, Re, Rb, and Cs, are separated by precipitation and ion exchange. Twelve 1-g samples can be

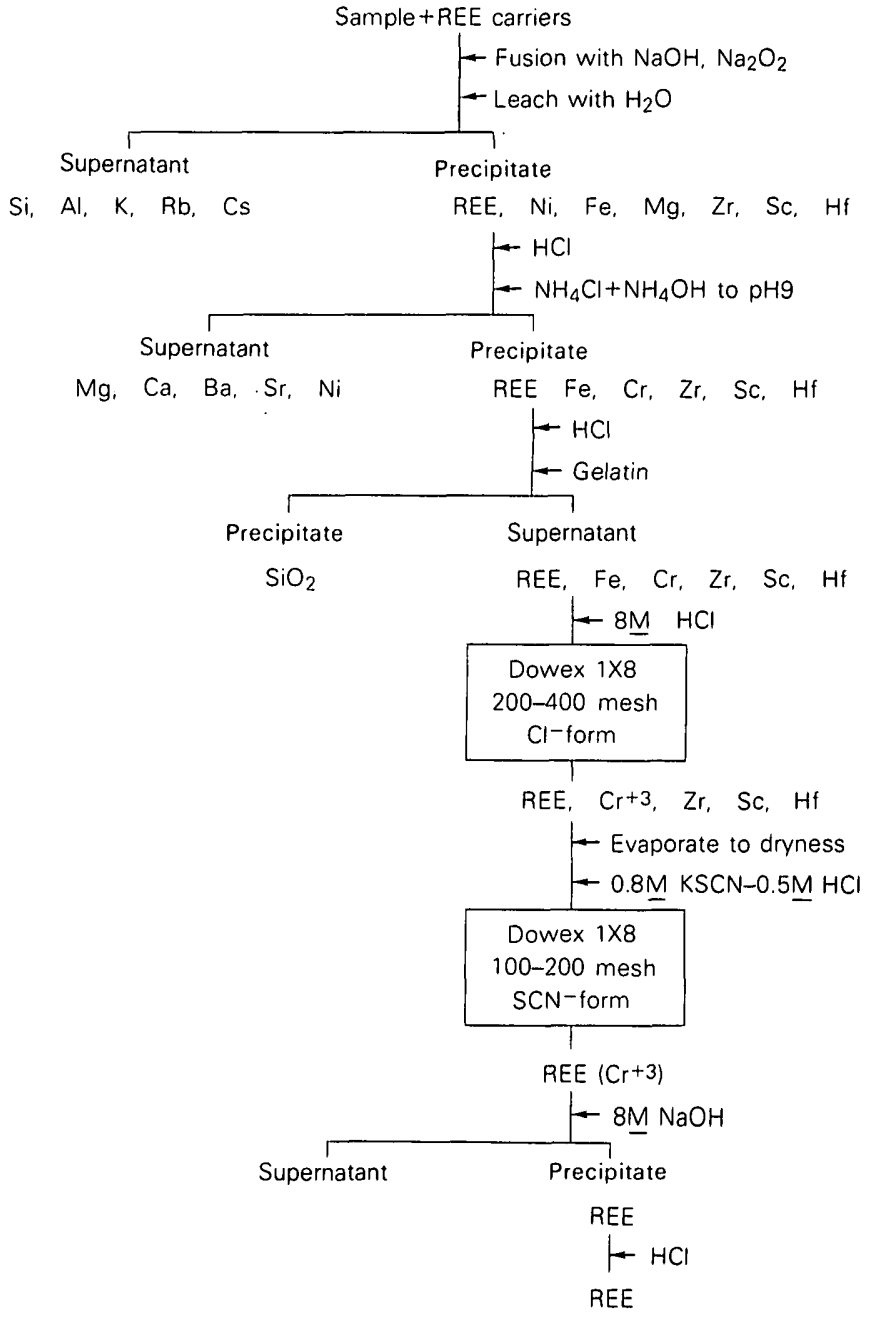

Figure 3. Group separation of the rare-earth elements used at the U.S. Geological Survey's National Center.

analyzed at a time for 19 elements at or below the part-per- billion level.

\section{Radioassay}

Measurement of the activity in the radiochemically processed samples is performed by gamma-ray spectrometry or beta counting. Gamma-ray spectrometry involves acquisition of spectra using coaxial $\mathrm{Ge}(\mathrm{Li})$ semiconductor detectors, planar low-energy photon detectors (LEPD), or $\mathrm{NaI}(\mathrm{Tl})$ scintillation detectors coupled to a multichannel analyzer. Each element is identified by its characteristic gamma-ray spectrum.

$\mathrm{NaI}(\mathrm{Tl})$ scintillation detectors have a relatively high efficiency, allowing detection of lower levels of radiation, but low resolution complicates analysis of complex spectra. $\mathrm{Ge}(\mathrm{Li})$ detectors have a lower efficiency but much better resolution allowing separation of close peaks in complex spectra. This allows detection of several elements in one sample after a group separation (for example, the 

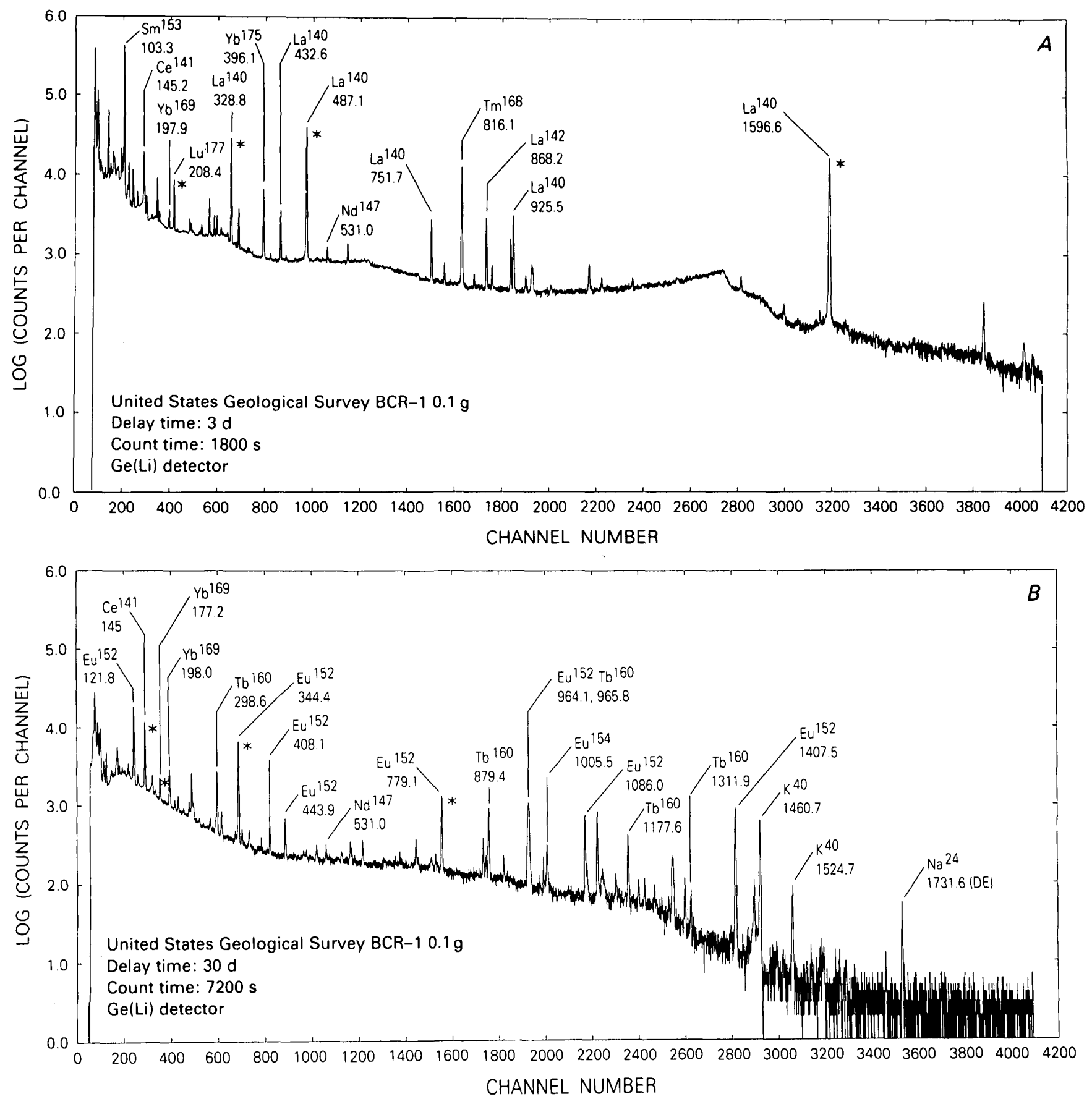

Figure 4. Gamma-ray spectrum of basalt BCR-1 after radiochemical neutron activation analysis separation of the rare-earth elements. $A$, Three days following neutron irradiation. $B$, Thirty days following irradiation. *, main analysis peaks; $D E$, double escape.

REE) and checking of radiochemical purity of processed samples. For detection and analysis of elements with low-energy gamma rays, the LEPD is best suited due to its higher resolution in the low-energy range; that is, up to $200 \mathrm{keV}$. Thus, the $84.3-\mathrm{keV}$ gamma ray of ${ }^{170} \mathrm{Tm}$ can be resolved from the $86.8-\mathrm{keV}$ gamma ray of ${ }^{160} \mathrm{~Tb}$ in spectra from REE group separation.

If no suitable gamma rays are available, then beta counting may be used for radioassay of those activation products that emit beta particles. A thin windowed gas

proportional counter or the Geiger-Muller tube commonly is used to detect beta particles. Because beta particles from a given nuclide are not monoenergetic, the energy spectrum cannot be used to resolve multiple beta emitters in the same same sample. Elements analyzed by beta counting, therefore, should be free of other beta emitters that would interfere. Radiochemical purity can be checked by testing for the exponential decay of beta activity over time because each isotope has a characteristic half-life. 
Once the activity of an element has been determined, the concentration of that element can be calculated using the following formulas:

$$
A_{m}=\frac{N_{m} e^{\lambda t}}{Y_{m} M_{m} C_{m}\left(1-e^{-\lambda T}\right) T}
$$

and

$$
A_{s}=\frac{N_{s} e^{\lambda t}}{Y_{s} M_{s}\left(1-e^{-\lambda T}\right) T},
$$

where

$A_{m}=$ specific activity of the monitor in counts per second per microgram of element,

$A_{s}=$ specific activity of the sample in counts per second per gram of sample,

$\mathrm{N}_{\mathrm{m}}=$ peak area or number of counts of the monitor,

$\mathrm{N}_{\mathrm{s}}=$ peak area or number of counts of the sample,

$\mathrm{t}=$ decay time in seconds,

$\mathrm{T}=$ count time in seconds,

$\mathrm{M}_{\mathrm{m}}=$ monitor weight in grams,

$\mathbf{M}_{\mathrm{s}}=$ sample weight in grams,

$\mathrm{Y}_{\mathrm{m}}=$ monitor chemical yield,

$\mathrm{Y}_{\mathrm{s}}=$ sample chemical yield,

$\mathrm{C}_{\mathrm{m}}=$ concentration of element in monitor in micrograms per gram, and

$\lambda=$ decay constant $=\ln 2 /$ half-life.

Therefore, the concentration, in micrograms per gram, of an element in the sample, $\mathrm{C}_{\mathrm{s}}$, is given by

$$
C_{s}=\frac{A_{s}}{A_{m}} \text {. }
$$

The exponential terms correct for decay before and during the counting process. For small values of $t$ or $T$, relative to the half-life of the indicator radionuclide, the corresponding exponential term can be ignored.

\section{Yield Determinations}

The goal of radiochemical separation is to provide the greatest possible decontamination of the radionuclides of interest from matrix interferences, not the optimization of recovery of those nuclides. Therefore, chemical yields must be determined to correct the counting data. Determination of the amount of carrier that remains after processing is the basis for the yield calculation. The common analytical methods described below are capable of determining milligram quantities and can be used to determine radiochemical yield.

Reactivation.-A portion of the processed sample is irradiated and analyzed for the desired elements. Because the carrier concentration is many times greater than that of the sample, the contribution by the sample to the gamma-ray spectra of the carrier elements is negligible. This method is used when suitable gamma-rays exist for the elements sought; examples are the REE, Ir, and In. Gravimetry.-A stable chemical compound of known stoichiometry containing the element of interest is weighed, and the amount of that element determined; examples are $\mathrm{Ni}$ as nickel dimethylgloxime and $\mathrm{Ag}$ as silver chloride.

Atomic absorption.-Processed samples are diluted to concentrations within the optimum range for atomic absorption analysis. This method commonly is used for $\mathrm{Ni}, \mathrm{Cu}, \mathrm{Zn}, \mathrm{Ga}, \mathrm{In}$, and $\mathrm{Au}$.

Radioactive tracer.-A known amount of radioactive tracer, not produced by the neutron activation of the sample, is added with the carrier before processing. After processing, the amount of tracer remaining is determined by gamma-ray spectroscopy; for example, in the determination of Cs, yields may be calculated by using ${ }^{137} \mathrm{Cs}$ as the tracer nuclide.

Energy-dispersive $X$-ray spectroscopy.-X-ray spectroscopy can be used for yield determinations of the REE. The chemically processed elements are collected on ion-exchange filter paper, and the amount of each REE is determined by X-ray fluorescence (Johnson and Wandless, 1983).

\section{DETECTION LIMITS, PRECISION, AND ACCURACY}

The sensitivity of RNAA is dependent on several factors, such as the isotopic abundance and neutron cross section of the target nuclide, half-life of the product nuclide, flux and energy spectrum of the neutron source, duration of irradiation, radiochemical purity and yield, and the efficiency and resolution of the counting equipment. Table 1 lists approximate detection limits for elements analyzed in our laboratories by RNAA. The elements grouped together in the table are often determined simultaneously by RNAA in a single irradiation.

The precision and accuracy for most of the elements shown in figure 2 is generally $1-10$ percent. Precision usually will decrease as elemental concentrations approach the detection limits, mainly due to poor counting statistics.

In Chapter $\mathrm{H}$, Baedecker and McKown have described several factors that influence the precision and accuracy of INAA and, in most cases, RNAA. Spectral interferences from the activation products of matrix elements are not a problem with RNAA because those radioisotopes have been removed. For group separations such as the REE, interferences on one REE from another do occur but are known and can be corrected for during data reduction. 


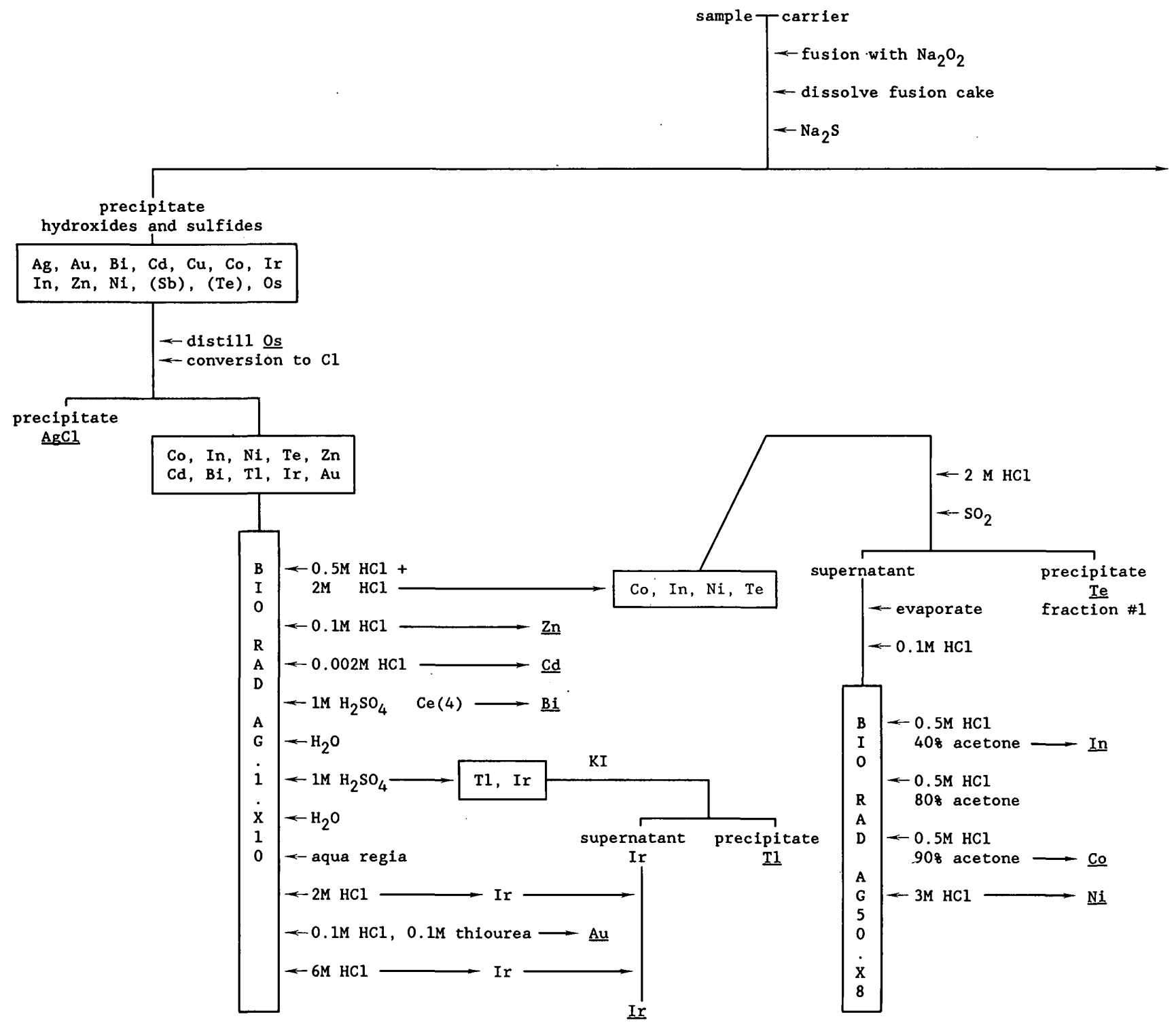

Explanation

underlined element symbol [for example Ir ] final fraction

element symbol in parentheses [for example $(R e)$ ] partial separation

Figure 5. Radiochemical neutron activation analysis of siderophile and volatile elements (from Keays and others, 1974). 


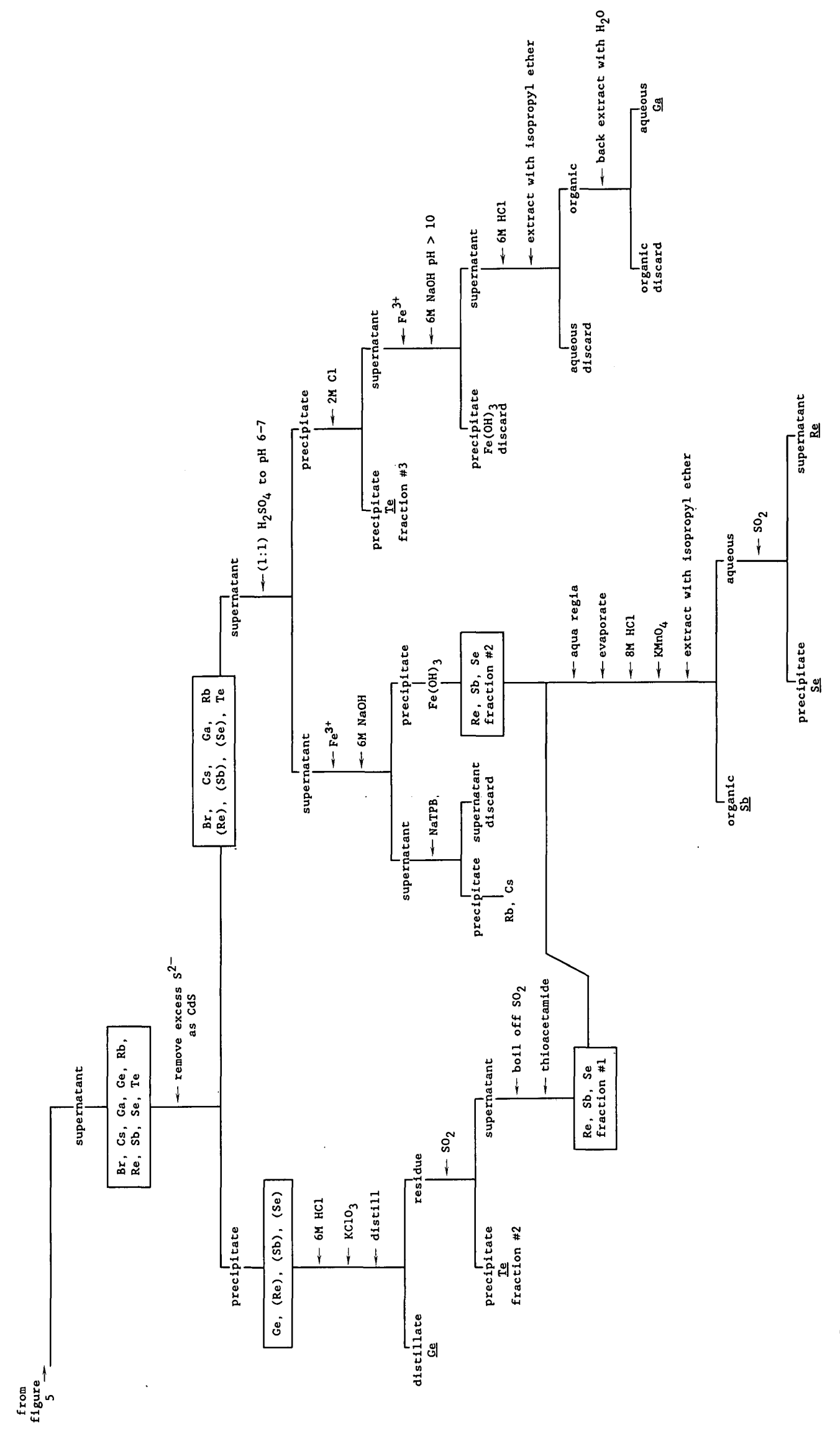


Table 1. Detection limits for various elements by radiochemical neutron activation analysis

[In nanograms per gram, except as noted]

\begin{tabular}{llll} 
Au & 0.001 & La & 1 \\
Ag & 0.01 & Ce & 5 \\
Bi & 0.05 & Nd & 5 \\
Cd & 0.01 & Sm & 0.5 \\
Ge & 1 & Eu & 0.1 \\
In & 0.1 & Gd & 5 \\
Ir & 0.001 & Tb & 0.1 \\
Ni & $5^{a}$ & Ta & 1 \\
Os & 0.005 & Yb & 5 \\
Pd & 0.1 & Lu & 0.1 \\
Re & 0.001 & & \\
Sb & 0.01 & Rb & 50 \\
Se & 0.1 & Cs & 1 \\
Te & 1 & & \\
TI & 0.1 & Ba & 50 \\
Zn & 10 & Sr & 200 \\
& & & \\
U & 1 & & \\
Th & 1 & & \\
\hline
\end{tabular}

\section{SUMMARY}

For determining abundances of many elements of geochemical interest, RNAA is an excellent technique. It provides the inherent sensitivity of neutron activation analysis by removing the matrix interferences before gamma-ray spectroscopy.

RNAA is a labor-intensive technique that limits the number of samples that can be analyzed but provides excellent data for favorable elements at levels that are inaccessible to most other methods. For chemically processing 12 samples, 1-8 weeks are required, and, for data acquisition and computations for the suites of elements listed in figures 3 and 5, 3-5 mo are required.

\section{REFERENCES CITED}

Baedecker, P. A., 1977, SPECTRA: Computer reduction of gamma-ray spectroscopic data for neutron activation analysis, in Taylor, R. E., ed., Advances in Obsidian Glass Studies: New Jersey, Noyes Press, p. 334-349.

Chou, C-L., 1980, Radiochemical neutron activation analysis, in Muecke, G. K., ed., Neutron activation analysis in the geosciences: Mineralogical Association of Canada Short Course Handbook, v. 5, p. 133-166.

Johnson, R. G., and Wandless, G. A., 1983, Determination of carrier yields for neutron activation analysis using energy dispersive X-ray spectrometry: Journal of Radioanalytical and Nuclear Chemistry, v. 81, p. 21-28.

Keays, R. R., Ganapathy, R., Laul, J. C., Krahenbuhl, U., and Morgan, J. W., 1974, The simultaneous determination of 20 elements in terrestrial, lunar, and meteoritic material by radiochemical neutron activation analysis: Analytica Chemica Acta, v. 72, p. 1-29.

Millard, H. T., and Bartel, A. J., 1971, A neutron activation analysis procedure for the determination of the noble metals in geological samples, in Brunfelt, A. O., and Steinnes, E. eds., Activation analysis in geochemistry and cosmochemistry: Proceedings of the NATO Advanced Study Institute, Oslo, Norway, Universitetsforlaget, $p$. 353-358.

Rammensee, W., and Palme, H., 1982, Metal-silicate extraction technique for the analysis of geological and meteoritic samples: Journal of Radioanalytical Chemistry, v. 71, p. 401.

Rowe, J. J., and Simon, F. O., 1968, The determination of gold in geologic materials by neutron activation analysis using fire assay for the radiochemical separations: U.S. Geological Survey Circular 599, p. 1-4.

Wandless, G. A., and Morgan, J. W., 1985, Analysis of low levels of rare earths by radiochemical neutron activation analysis, I: Journal of Radioanalytical and Nuclear Chemistry, v. 92 , p. 273-282. 


\section{CHAPTER $\mathrm{K}$}

\section{Isotope-Dilution Mass Spectrometry}

By J. A. PHILPOTTS

U.S. GEOLOGICAL SURVEY BULLETIN 1770

Methods for Geochemical Analysis 



\title{
CONTENTS
}

\author{
Abstract K1 \\ Introduction $\mathbf{K} \mathbf{1}$ \\ Basic principles of isotope dilution $\mathbf{K} \mathbf{1}$ \\ Surface ionization mass spectrometry $\mathbf{K 2}$ \\ The mass spectrum $\mathbf{K 3}$ \\ Sample-preparation chemistry $\mathbf{K} 3$ \\ Analytical quality $\mathbf{K 3}$ \\ Limitations of the technique K4 \\ Availability of isotope-dilution analysis $\mathrm{K} 4$ \\ Acknowledgments K4 \\ References cited K5
}




\title{
Isotope-Dilution Mass Spectrometry
}

\author{
By J. A. Philpotts
}

\begin{abstract}
Isotope-dilution mass spectrometry utilizes isotopes of the element(s) of interest as internal standards to provide abundance determinations of exceptional quality. About 50 elements are potentially amenable to analysis using solidsource mass spectrometry, but the method is particularly valuable for determining abundances of trace elements, including alkalis, alkaline earths, and rare earths. The technique is applicable to any material that can be put in solution. Typically, for silicates, samples of about 100 milligrams are dissolved in hydrofluoric acid. The technique is relatively insensitive to the matrix, although some separation chemistry is normally desirable. However, multielement determinations are often convenient. Complete chemical yield is not required during processing once isotopic equilibration has been achieved. Typically, precision and accuracy are a few percent at part-per-million levels. On the debit side, the technique is relatively laborious, slow, and expensive.
\end{abstract}

\section{INTRODUCTION}

Isotope-dilution mass spectrometry (MSID) offers the highest quality abundance determinations available for many of the trace elements. The technique provides excellent sensitivity, precision, and accuracy. MSID benefits from the use of ideal internal standards, namely isotopes of the element being determined, and does not require complete chemical yield once the internal standard has been mixed with the sample. About 50 elements are potentially amenable to analysis by MSID using a solid-source mass spectrometer. The technique is particularly valuable for the determination of elements present in trace amounts. Contamination is the major limiting factor. The technique is also relatively slow and expensive, and its availability is limited.

\section{BASIC PRINCIPLES OF ISOTOPE DILUTION}

A comprehensive discussion of MSID has been given by Webster (1960). The isotope-dilution procedure is based on the measurement of the isotopic composition of an element of interest in a mixture composed of the unknown sample and a known amount of a tracer having distinctly different isotopic composition for the element(s) than has the unknown. The isotopically enriched tracer, commonly termed a. "spike," is normally a synthetic compound (such as those produced by the Oak Ridge National Laboratory, Oak Ridge, Tennessee) although natural materials have been used in some instances. The isotopic composition of the element measured for the mixture will be a function of the isotopic composition and the amount of the element in both components of the mixture. The isotopic composition of the element in the unknown can be obtained by a separate isotope-ratio measurement, but, usually, it is adequate to assume "normal" isotopic composition in natural samples. An isotope-balance equation is solved for the only unknown parameter, namely the amount of the element of interest in the unknown sample; for example, if $R$ is the abundance ratio of "mass" (actually mass number) 7 to mass 6 measured on the mass spectrometer for a mixture of normal sample and spike lithium $(\mathrm{Li})$, then

$$
R=\frac{N\left(\%^{7} \mathrm{Li}\right)_{\mathrm{N}}+\mathrm{S}\left(\%^{7} \mathrm{Li}\right)_{S}}{\mathrm{~N}\left(\%^{6} \mathrm{Li}\right)_{\mathrm{N}}+\mathrm{S}\left(\%^{6} \mathrm{Li}\right)_{\mathrm{S}}}
$$

where $\mathrm{N}$ is the amount of normal lithium in the sample, and $S$ is the known amount of lithium added by the spike, both expressed as atoms or as moles. Inasmuch as the percentages of ${ }^{7} \mathrm{Li}$ and ${ }^{6} \mathrm{Li}$ are known in both components and $\mathrm{R}$ is a measured ratio, $\mathrm{N}$ is the only unknown. Equation 1 may be rearranged to

$$
\frac{\mathrm{N}}{\mathrm{S}}=\frac{\left(\%^{7} \mathrm{Li}\right)_{\mathrm{S}}-\mathrm{R}\left(\%^{6} \mathrm{Li}\right)_{\mathrm{S}}}{\mathrm{R}\left(\%^{6} \mathrm{Li}\right)_{\mathrm{N}}-\left(\%^{7} \mathrm{Li}\right)_{\mathrm{N}}} .
$$

As given above, N/S is an atomic ratio. Concentration of the element in the unknown in terms of parts per million by weight is given by

$$
\begin{gathered}
\text { ppm }=\frac{N}{S} \cdot \frac{\text { (normal } \mathrm{Li} \text { atomic wt.) }}{\text { (spike Li atomic wt.) }} \\
\frac{(\mathrm{ml} \text { of spike) }(\mu \mathrm{g} \text { of element } / \mathrm{ml} \text { of spike) }}{\text { (weight of sample in } \mathrm{g})} .
\end{gathered}
$$

Although the technique is often referred to as "stable" isotope dilution, it is quite applicable for deter- 
mining abundances of radioactive elements, such as uranium. The only basic requirement is that an element have at least two relatively stable isotopes. Hence, isotope dilution can be used in determining abundances of elements that are monoisotopic in nature, providing that suitably long-lived radioactive spikes are available. Beryllium, cesium, iodine, and thorium are examples of monoisotopic elements that can be determined by isotope dilution. Monoisotopic elements that are not suitable are fluorine, sodium, aluminum, phosphorus, scandium, manganese, cobalt, arsenic, yttrium, niobium, rhodium, praseodymium, terbium, holmium, thulium, gold, and bismuth. This still leaves about 70 elements. In principle, isotope dilution may be employed with any analytical technique sensitive to isotopes, but, in practice, it is usually coupled with mass spectrometry. Further limitations on what elements may be analyzed arise from this choice.

\section{SURFACE IONIZATION MASS SPECTROMETRY}

A mass spectrometer separates ions on the basis of their mass to charge ratio and their velocity as they pass through an electrical or magnetic field to an appropriate detector. (For a brief discussion, see, for example, Faure, 1977 , p. 65.) For separation in a magnetic field, the relation is

$$
\mathrm{r}=\frac{\mathrm{mu}}{\mathrm{Be}},
$$

where $r$ is the radius of curvature of the collimated ion beam, $m$ is the mass of the ion, $e$ is the ion's electronic charge, $B$ is the magnetic field strength, and $u$ is the velocity. The velocity is acquired before entering the magnetic field by accelerating the ions through a potential difference, $\mathrm{V}$, according to

$$
\mathrm{u}=\left\{\frac{2 \mathrm{e} \mathrm{V}}{\mathrm{m}}\right\}^{1 / 2}
$$

Combining equations 4 and 5 yields

$$
\frac{\mathrm{m}}{\mathrm{e}}=\frac{\mathrm{B}^{2} \mathrm{r}^{2}}{2 \mathrm{~V}}
$$

This is the basic equation for magnetic sector mass spectrometry. For a particular magnetic field strength (B) and accelerating potential (V), the path ( $r$ ) of ions through the field is specific for any mass to charge ratio. Ions of interest may be tuned into the detector by changing the magnetic field strength or the accelerating potential. It might be noted that this relation is given incorrectly in Faure (1977, p. 67, eq 5.4).

For gases and readily volatilized species, ions may be produced by electron bombardment. Another way of producing ions involves thermal emission from resistanceheated filaments. This type of ion production (called surface ionization or solid source) is more appropriate for most of the elements, particularly when they are in trace quantities, inasmuch as it results in a cleaner mass spectrum (that is, one with less mass interference), especially when used in conjunction with a liquid-nitrogencooled cold finger in the source region. The ionization efficiency of a solid source, expressed in terms of the ratio of positive ions $\left(\mathrm{n}^{+}\right)$to neutral atoms $\left(\mathrm{n}^{\circ}\right)$ leaving the filament, is given by the relation,

$$
\frac{\mathrm{n}^{+}}{\mathrm{n}^{\circ}} \alpha \exp \left\{\frac{\mathrm{e}(\mathrm{W}-\Phi)}{\mathrm{k} \mathrm{T}}\right\},
$$

where $\mathrm{W}$ is the surface work function of the filament material, $\Phi$ is the ionization potential of the element of interest, $\mathbf{k}$ is Boltzmann's constant, and $\mathbf{T}$ is absolute temperature. Clearly, ion production is favored by high work function and low ionization potential. When ionization potential exceeds work function, maximum efficiency is obtained with temperature as high as possible. Most of the metals of interest as filament material have work functions of about $5 \pm 0.5 \mathrm{eV}$. Platinum is high at about $5.7 \mathrm{eV}$ but has the disadvantage, when used for refractory samples, of melting at a relatively low $2042 \mathrm{~K}$. Rhenium $(5.0 \mathrm{eV}, 3450 \mathrm{~K})$ is often used as filament material. It might be noted that the exact nature of the chemical reactions occurring on the filaments is not yet fully understood, and heuristics still play a major role in defining mass-spectrometric procedures. Reactions of sample with filament will modify the work function. Also, a multiplicity of chemical species of various volatility and ionization potential may be produced.

Although high temperature can increase surface ionization efficiency, a too-rapid loss of sample in the case of volatile materials is a potential problem. One means of reducing this difficulty is to have the sample loaded on a filament separated from the high-temperature ionizing filament. These double-filament and triple-filament sources generally give greater control over ion production and result in more stable ion beams and less interelement isobaric interference. Another approach to the problem of high volatility is to load the sample on to the filament with a carrier phase. One such technique, first used to improve analysis of lead, a relatively volatile element, involves loading the sample on a V-shaped filament along with silica gel and phosphoric acid (Cameron and others, 1969). Ion yields have improved with these techniques to the point that high volatility and high ionization potential essentially are no longer limiting factors in thermal ionization, although elements of low ionization potential do produce much larger signals in the mass spectrometer. Unless a suitable volatile form can be found, highly refractory elements such as tungsten, molybdenum, tantalum, and osmium generally do not provide adequate 
signal and are better ionized by using ion sputtering, plasma, or other sources. In all, there are about 50 elements for which thermal-ionization massspectrometric isotope-dilution analysis is feasible. The technique is particularly well suited for determinations of alkali metals, alkaline earths, and rare earths, especially when these elements are present in trace amounts (Schuhmann and Philpotts, 1979).

\section{THE MASS SPECTRUM}

Ideally, a mass spectrum reflects solely the isotopic abundances of the element of interest. The measured isotopic composition may differ from the actual composition of the sample-spike mix. One possible effect is mass-dependent fractionation in the source, particularly for light elements. In practice, this effect is rarely significant and may be reduced by using triple filaments, by integrating the signal over extended time, and by measuring heavier compound molecular ions (for example, $\mathrm{LiNO}_{3}{ }^{+}$rather than $\mathrm{Li}^{+}$) so as to lessen relative mass differences. Mass-spectrometer bias, such as that caused by differential collection efficiency, is also rarely important in isotope-dilution abundance determinations; such fractionation is lessened if the mass spectrum is scanned electromagnetically at constant ion-acceleration potential and, in any case, may be self cancelling. The technique of double spiking offers another means of correcting instrument fractionation in the case of elements with more than two isotopes.

In practice, the mass spectrum is rarely element specific. A certain amount of contamination is inevitable; at low levels, contamination can come from the massspectrometer itself. Furthermore, it is frequently convenient to determine abundances of several elements from the same filament loading. In addition, good analyses usually may be made without prior concentration of samples to high degrees of purity because isotope dilution is considerably less matrix sensitive than most analytical techniques. However, the presence of additional elements in the sample loaded on the filament may complicate the mass spectrum to some extent. One possible effect is isobaric interference of ions having the same mass to charge ratio. Use of triple filaments reduces the occurrence of isobaric interference. Some interference may be avoided by measuring a different ionic species in another region of the mass spectrum. Thus, interference of ${ }^{156} \mathrm{CeO}^{+}$(that is, ${ }^{140} \mathrm{Ce}+{ }^{16} \mathrm{O}$ ) on ${ }^{156} \mathrm{Gd}^{+}$may be avoided by analyzing ${ }^{172} \mathrm{GdO}^{+}$, for example, providing ${ }^{172} \mathrm{Yb}^{+}$ presents no problem. The proportion of $\mathrm{GdO}^{+}$to $\mathrm{Gd}^{+}$ may be increased by oxidizing the sample during filament loading or by in-leakage of oxygen during massspectrometric analysis among other techniques. Conversely, $\mathrm{GdO}^{+}$interference on $\mathrm{Yb}^{+}$may be reduced by in-leakage of propane or hydrogen during the massspectrometric analysis while maintaining a vacuum of about $10^{-6}$ torr (that is, about $0.1 \mathrm{mPa}$ ) or better or by mixing a reducing agent such as carbon in with the sample loaded on the filaments (Schuhmann and others, 1980). When isobaric interference does occur, mass spectra are usually simple enough that the interference is obvious by inspection, by a correlation of calculated abundance with measurement order as interfering species undergo differential signal growth or decay, and by discrepancies between abundances calculated from different isotope pairs for elements with more than two isotopes. Furthermore, it is usually possible to effectively remove the effect of isobaric interference by applying corrections during the calculation of abundance; this correction is particularly easy if the interfering elements have individual isotopes that are interference free.

The mass spectrum can be affected in other ways from the presence of additional elements on the filaments. In particular, readily ionizable species present in abundance can cause baseline (background) problems and also result in suppression of the signal for the element of interest. Because of problematic isobaric interference and other factors, including general instrument cleanliness, some degree of element concentration and purification generally is performed during sample preparation.

\section{SAMPLE-PREPARATION CHEMISTRY}

The isotope-dilution technique requires complete mixing of sample and spike. For solid samples, this necessitates complete decomposition. Silicate samples typically are decomposed in hydrofluoric acid. Digestion in an open system has the added advantage of removing silicon, normally an abundant element, as the volatile fluoride $\left(\mathrm{SiF}_{4}\right)$. Normally, the spike is added during dissolution. An attractive feature of isotope dilution is that, once equilibration of sample and spike has been achieved, complete quantitative chemical yield is no longer necessary, it is only the ratio of $N$ to $S$ (see eqs 2,3 ) that must be determined, not their absolute abundances. In practice, high yields are preferable to reduce the chance of incomplete mixing of sample and spike, to lessen the possibility of isotopic fractionation in any residual precipitate, and to keep the effect of subsequent contamination to a minimum. Once a sample is in solution and equilibrated with spike, then partial separation, at least by groups of elements, generally is performed using ionexchange chromatography, solvent extraction, precipitation, or other techniques.

\section{ANALYTICAL QUALITY}

The quality of abundance determinations using solid-source mass-spectrometric isotope dilution may be 
considered in terms of sensitivity, precision, and accuracy. The theoretical sensitivity of the technique is a function of isotopic composition of the element in the sample and the spike. Miniaturization of chemical procedures and stateof-the-art mass spectrometry together have produced remarkable results. Kelly and others, (1978), for example, reported a detection limit of about $1 \mathrm{fmol}$ for silver, an element with ionization efficiency of only a percent or two. Inherent sensitivity is rarely a limiting factor with the technique. Contamination by reagents, lab ware, the atmosphere, and so forth, usually dictates the lower limits of measurement. Actual sensitivities of about a nanogram of an element or a part per billion in normal samples are fairly typical. For low-level work, clean rooms are warranted. Because of the problem of contamination, MSID may be an inferior technique to neutron activation analysis for very small samples or low abundances.

Precision (random error) is also a function of the isotopic composition of the element in the unknown and the spike and the proportions of both components in the mix. Precision is optimized when the isotopic ratio in the mix is the geometric mean of the ratios in both end members. The use of composite spikes to facilitate multielement determinations will tend to degrade precision somewhat because of nonoptimum mix ratios, in general, and less-enriched spike owing to interspike cross contamination. Typical precisions range from a fraction of a percent to about 5 percent at part-per-million levels, depending on the element, instrumentation, contamination, and so forth. Kelly and others (1978) reported determining $0.5 \mathrm{pmol}$ of silver (about $50 \mathrm{pg}$, or $3 \times 10^{11}$ atoms) with a precision of better than 1 percent.

Accuracy (systematic error) may be affected by instrument fractionation, but this is normally a small effect. When the concentration of the spike is calibrated against gravimetric standards, the absolute accuracy of the technique should be comparable to the precision, which is typically in the range of $1-5$ percent.

\section{LIMITATIONS OF THE TECHNIQUE}

Major advantages of MSID are the high quality of the determinations at part-per-million or lower levels and the sample preparation chemistry not requiring quantitative chemical yield. The technique is limited to elements with a minimum of two appropriately stable isotopes. Limitations of MSID have been discussed above in terms of ionization potentials and volatilities. Other limitations are as follows. The technique is destructive of the material analyzed although samples are ordinarily relatively small (about $100 \mathrm{mg}$ ). Complete solid sample decomposition and equilibration with spike are required, and both may be difficult in particular instances; for example, lead in natural gold. Chemical preparation of samples, including separations, is more involved than is the case for many other analytical techniques and typically may take 2 or 3 d per sample set. On all but the most modern mass spectrometers, vacuum has to be broken to load each individual sample, and this necessity is time consuming. From this perspective, it is advantageous to determine abundances of a number of elements per filament loading, if this is convenient. Typical mass spectrometer run times are on the order of $1 \mathrm{~h}$ per element per sample, and this is slow compared with many other techniques.

A major limitation of MSID is cost. Mass spectrometers tend to be expensive items of equipment to purchase (about $\$ 250,000$ for leading models) and to maintain. Furthermore, mass spectrometers in use for highprecision isotope-ratio studies may not be available for isotope-dilution analyses because of possible contamination; for example, Rb may not be permitted in an instrument used for $\mathrm{Sr}$ isotope-ratio work because mass 87 is common. Mass-spectrometric analysis, particularly on older nonautomated instruments, is labor intensive. Inasmuch as the detailed run characteristics of multielement determinations can be difficult to predict and, hence, to preprogram, the interaction of a spectroscopist may be required during analysis even with automated equipment. Finally, it might be noted that some of the artificially enriched isotopic spikes can be expensive if used in quantity and, at times, may not be available.

\section{AVAILABILITY OF ISOTOPE-DILUTION ANALYSIS}

Currently MSID is not provided as a routine service of the Geologic Division of the U.S. Geological Survey. Abundances of some elements are sometimes determined by MSID in the Branch of Isotope Geology in the course of geochronologic and isotope-tracer studies. Commercial geochronology laboratories also may offer MSID for a few elements.

A recently acquired inductively coupled plasma mass spectrometer combines the capability of the inductively coupled plasma source for conveniently handling large numbers of samples with the isotope-dilution capability of a quadrupole mass spectrometer. In addition, the plasma source may ionize refractory species not handled adequately by thermal surface ionization. This type of instrument may prove suitable for routine MSID analytical support.

\section{ACKNOWLEDGMENTS}

The manuscript has benefited from useful comments by Joseph Arth, Bruce Doe, Shuford Schuhmann, and Herman Thomas. 


\section{REFERENCES CITED}

Cameron, A. E., Smith, D. H., and Walker, R. L., 1969, Mass spectrometry of nanogram-size samples of lead: Analytical Chemistry, v. 41 , no. 3 , p. 525-526.

Faure, Gunter, 1977, Principles of isotope geology: New York, John Wiley and Sons, 464 p.

Kelly, W. R., Tera, Fouad, and Wasserburg, G. J., 1978, Isotopic determination of silver in picomole quantities by surface ionization mass spectrometry: Analytical Chemistry, v. 50 , no. 9 , p. $1279-1286$.
Schuhmann, Shuford, and Philpotts, J. A., 1979, Massspectrometric stable-isotope-dilution analysis for lanthanides in geochemical materials, in Gschneider, K. A., Jr., and Eyring, LeRoy, eds., Handbook on the physics and chemistry of rare earths, v. 4, Non-metallic compounds II: Amsterdam, North-Holland, p. 471-481.

Schuhmann, Shuford, Philpotts, J. A., and Fryer, Patricia, 1980, Suppression of monoxide interference in surface ionization mass spectrometry of rare-earth elements: Analytical Chemistry, v. 52, no. 1, p. 214-216.

Webster, R. K., 1960, Mass spectrometric isotope dilution analysis, in Smales, A. A., and Wager, L. R., eds., Methods in geochemistry: London, Interscience, p. 202-246. 